GEOLOGICAL SURVEY CIRCULAR 295

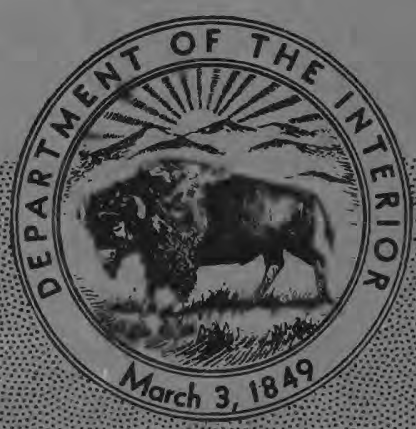

IRRIGATION-WELL DEVELOPMENT IN THE KANSAS RIVER BASIN OF EASTERN COLORADO 
UNITED STATES DEPARTMENT OF THE INTERIOR

Douglas McKay, Secretary

GEOLOGICAL SURVEY

W. E. Wrather, Director

GEOLOGICAL SURVEY CIRCULAR 295

\section{IRRIGATION-WELL DEVELOPMENT IN THE KANSAS RIVER BASIN OF EASTERN COLORADO}

By W. D. E. Cardwell 


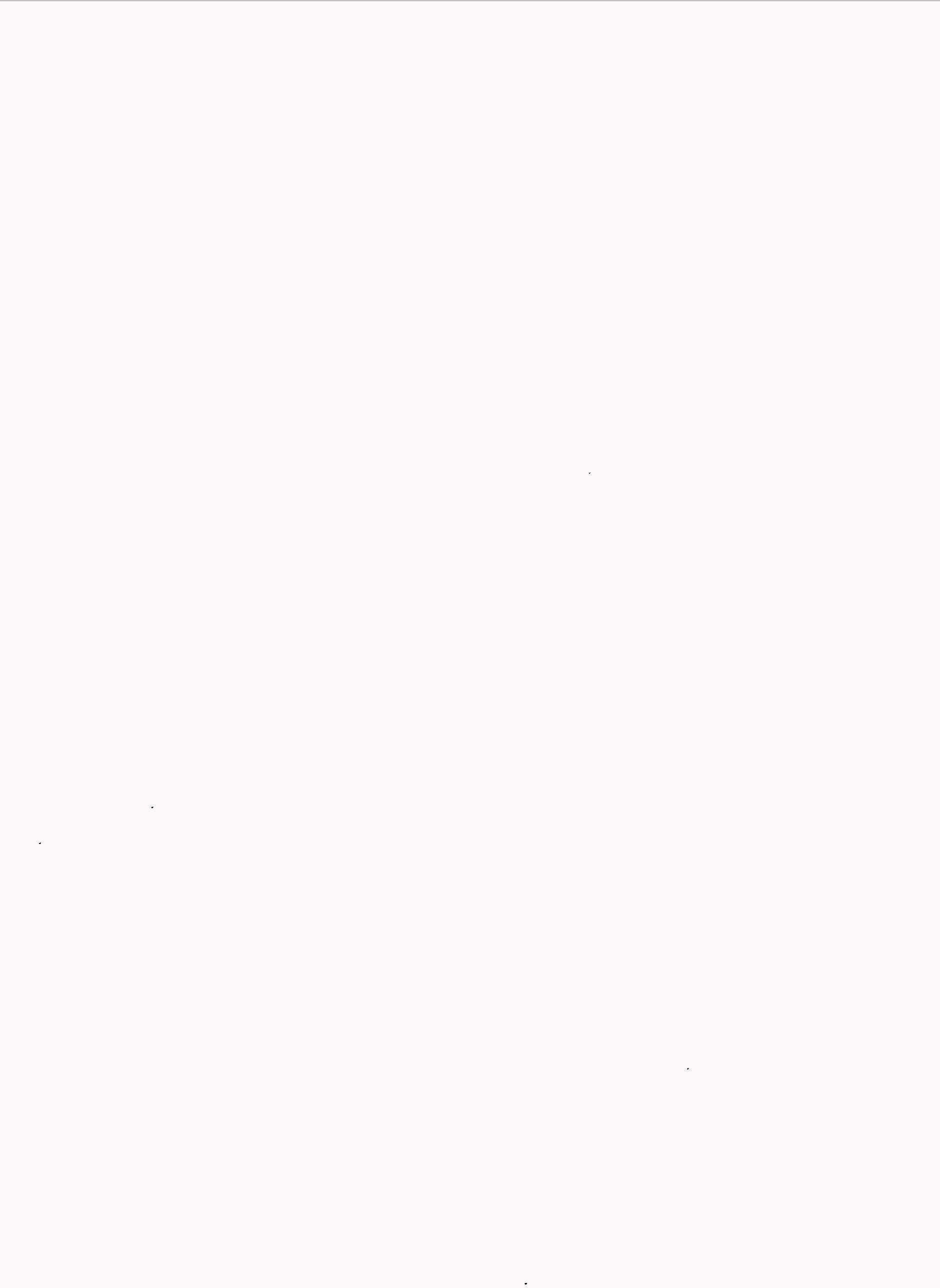


Plate 1. Depth to water in part of eastern Colorado and the location of wells for which

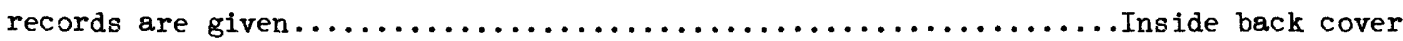

2. Configuration of the erosion surface on the Pierre shale in parts of Cheyenne,

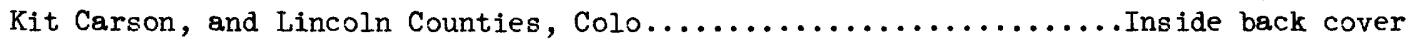

3. Fence diagram of part of Kit Carson County, Colo................. Inside back cover

Figure 1. Map of the Missouri River basin showing areas in which ground-water studies have been made under the program for the development of the Missouri

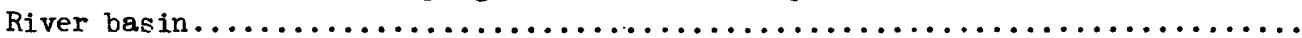

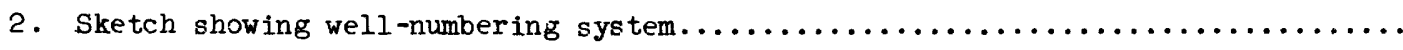

3. Diagrammatic sections showing the relationship of a stream to the water table...

4. Annual precipitation at Akron, Arriba, Burlington, Cheyenne Wells, LeRoy,

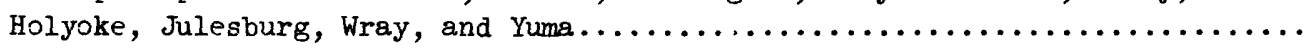

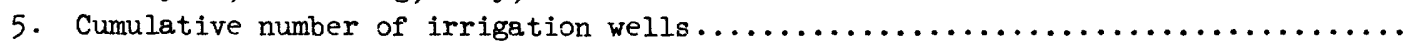

\section{TABLES}

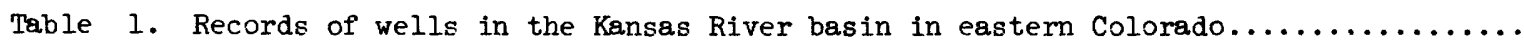




\section{•}




\title{
IRRIGATION-WELL DEVELOPMENT IN THE KANSAS RIVER BASIN OF EASTERN COLORADO
}

\author{
By W. D. E. Cardwell
}

ABSTRACT

The Kansas River basin in eastern Colorado consists mainly of flat upland plains mantled in places by sand hills and dissected only by the valleys of the main streams. In this semiarid region, which has an average annual precipitation of about 17 inches, farming and the raising of livestock are the principal occupations. Irrigation, which has increased greatly since 1940, is practiced in several places in the region.

The exposed rocks in the region are of Cretaceous to Recent age. Much of the region is underlain by the Ogallala formation of Tertiary (Pliocene) age. The Sanborn formation of Pleistocene and Recent(?) age overlies the Ogallala formation in the southeastern part of the region, and the Pierre shale of Cretaceous age, which underlies the Ogallala formation throughout the region, crops out in the more deeply incised valleys. Many parts of the upland area are underlain by dune sand, and the principal valleys are underlain by alluvium of Quaternary age. The Ogallala formation contains thick beds of sand and gravel, which yield quantities of water sufficient for irrigation in many places. The slope of the surface of the Pierre shale is about 33 feet per mile to the north-northeast.

The ground water reservoir is recharged principally by precipitation that falls with- in the region and by percolation from intermittent streams and depressions. Ground water is discharged from the ground-water reservoir by movement into adjacent areas to the east and southeast, by evaporation and transpiration in areas of shallow water table, by seepage into perennial streams, and by wells. All water used for domestic, stock, public, and industrial purposes and most of the water for irrigation is obtained from wells.

Most of the wells in the area are drilled. In 1950, 66 of the wells supplied water to irrigate 5,760 acres. The areas most favorable for the development of irrigation are southeast of Holyoke, Phillips County; southeast of Burlington, Kit Carson County; and the valley of the Arikaree River in the vicinity of Cope, Washington County.

The ground water from the Ogallala formation is moderately hard but is suitable for most uses. Water from the alluvium of Recent age generally is much harder.

The field data upon which most of this report is based are given in tables, which include records of 178 wells and a compilation of data on irrigation wells. Logs of 329 test holes, water wells, and seismograph shot holes are included in the report. 


\section{INTRODUCTION}

Purpose of the investigation

This investigation was begun by the United States Geological Survey at the request of the United States Bureau of Reclamation as a part of the program of the Interior Department for development of the Missouri River basin. The investigation was endorsed also by the Colorado State Water Conservation Board.

Ground water is one of the important natural resources of eastern Colorado, and each year more irrigation wells are constructed for its further utilization. Irrigation wells are concentrated locally in widely separated areas; significantly, however, individual wells of large yield are scattered between the areas of concentration. (See pl.1.)

The pumping of ground water for irrigation in this area is increasing rapidly. Although pumping at the present rate probably would not result in a significant regional lowering of the water table, pumping at a doubled or trebled rate, which is not improbable, would cause both a lowering of the water table and a reduction in the base flow of perennial streams. A lowering of the water table probably would result also in greater infiltration to the ground-water reservoir of flood waters in the upper reaches of intermittently flowing streams. Although this recharge might not materially increase yields from wells in eastern Colorado, it probably would reduce appreciably the direct stream discharge into the surface reservoirs that have been built by the Bureau of Reclamation on the lower reaches of these streams.

All known irrigation and public-supply wells in the area were inventoried during this investigation and the resulting data have been tabulated. This information will be useful in the planning of new irrigation wells and as a basis for comparison when the effects of the additional pumping are known. Areas in which puaping of ground water for irrigation is feasible and areas in which it is not feasible have been delineated.

\section{Location and extent of region}

The region includes all the Kansas River basin in Colorado. It extends southward from the South Platte Valley to Cheyenne Wells and westward from the Colorado State line to Fleming in the north and Genoa in the south. (See fig. 1.) It includes all of Kit Carson, Yuma, and Phillips Counties and parts of Cheyenne, Elbert, Lincoln, Logan, Sedgwick, and Washington Counties. The region, which is about 150 miles north and south and averaging about 60 miles east and west, contains about 9,000 square miles.

\section{Methods of investigation}

The investigation was begun in July 1950 under the general supervision of A. N. Sayre, chief of the Ground Water Branch of the U.S. Geological Survey, and of G. H. Taylor, regional engineer in charge of the ground-water investigations in the Missouri River basin, and under the immediate supervision of T. G. McLaughlin, acting district geologist, Denver, Colo. The field work was completed in December 1950.

Measurements of the water level in 22 wells scattered throughout the region were made monthly. Most of the observation wells are in the areas of greatest concentration of irrigation wells.

of the 178 wells included in the inventory, 121 are or are to be irrigation wells, 41 are public supply wells, 12 are abandoned domestic or stock wells used as water-level observation wells, 3 are stock wells and 1 is a cemetery well. (See table 1.) When possible, the total depth of the well and the depth to the static water level were measured using a steel tape; the total depth and the depth to the static water level in other wells were reported by the well owner or driller.

The discharge of 21 irrigation wells was measured using a Hoff current meter. Well owners and drillers reported the character 


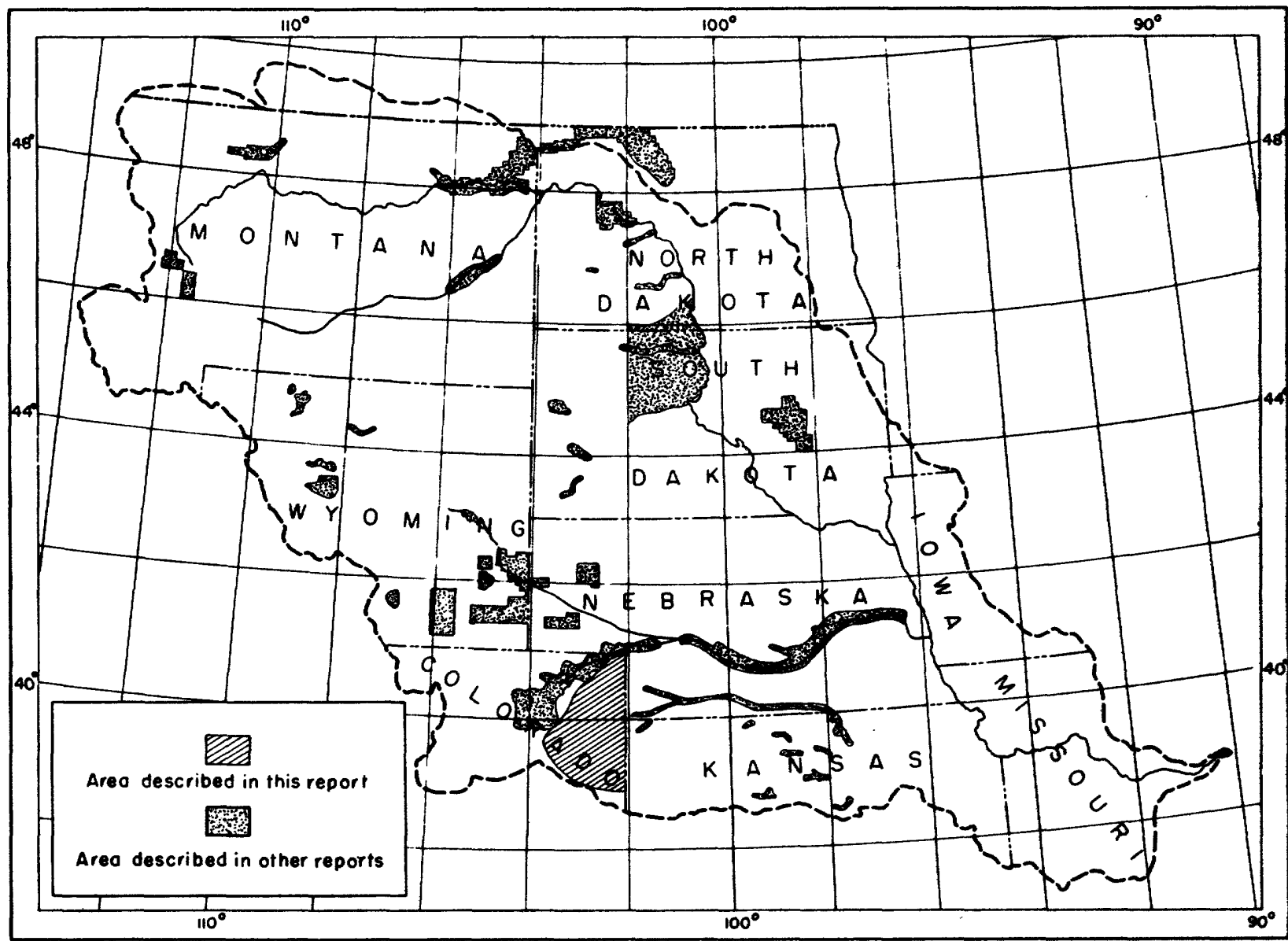

Figure 1. Map of the Missouri River basin showing areas in which ground-water studies have been made under the program for the development of the Missouri River basin.

and thickness of water-bearing formations, methods of well construction, type of pumping plants, irrigation methods, irrigated acreages, and other pertinent data. Well locations were determined by odometer measurements or by pacing from section corners.

All available well logs were collected from owners of irrigation wells, well drillers, municipalities, railroads, and oil companies. Many logs of test holes and of seismograph shot holes penetrating to the Pierre shale were compiled. (See table 2.)

\section{Well-numbering system}

Well numbers appearing in this report are based on the system of land subdivision of the Bureau of Land Management. The well number shows the location of the well by township, range, section, and position within the section. The first letter (capital) of a well number gives the quadrant of the meridian and baseline system in which the well is located; the letters begin with $A$ in the northeast quadrant and proceed counterclockwise. All 
wells in this area lie in the northwest (B) or southwest (C) quadrants of the sixth principal meridian and baseline system. The first numeral of a well number indicates the township, the second indicates the range, and the third indicates the section in which the well is located. The lowercased letters following the section number locate the well within the section. The first letter denotes the quarter section and the second letter denotes the quarter-quarter section. The letters are assigned in a counterclockwise direction beginning with (a) in the northeast quarter of the section or of the quarter-quarter section. For example, the well number $\mathrm{Bl}-45-30 \mathrm{bd}$ indicates a location in the $\mathrm{SE}_{\frac{1}{4}} \mathrm{NW} \frac{1}{4} \mathrm{sec} .30, \mathrm{~T}$. $1 \mathrm{~N} ., \mathrm{R} .45 \mathrm{~W}$. Where more than one well is located in a quarter-quarter section, consecutive numbers beginning with 1 are added. A graphical illustration of this method of well numbering is shown in figure 2 .

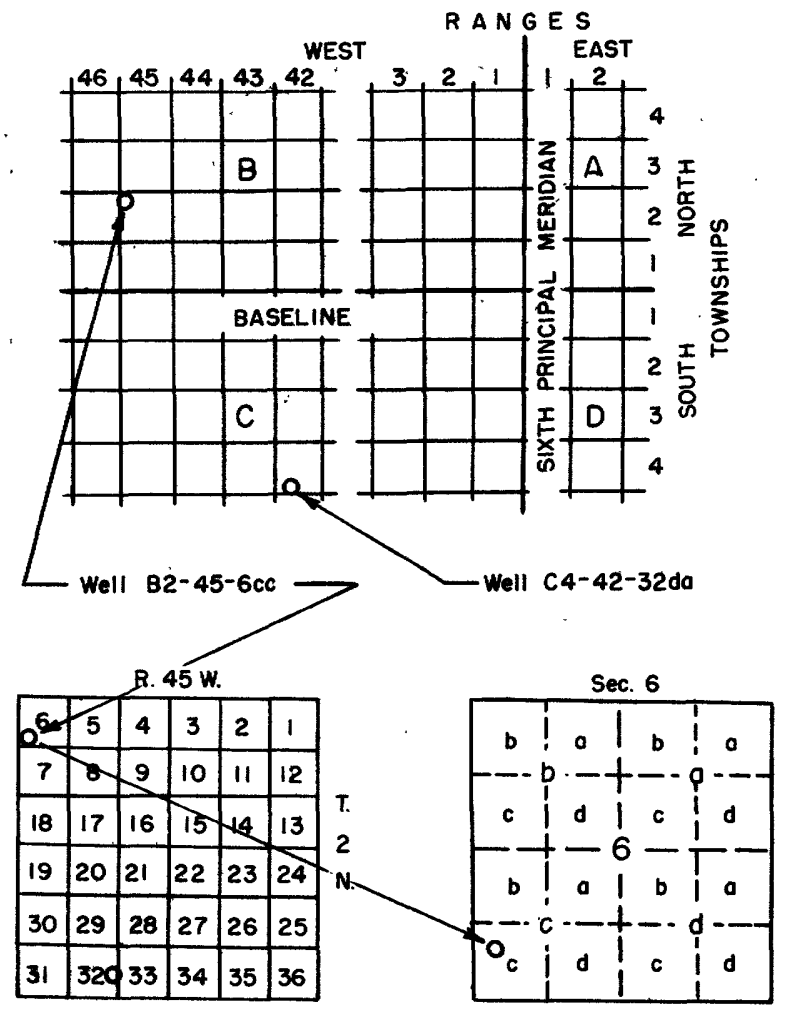

Figure 2.--Sketch showing well-numbering system.

\section{Acknowledgments}

Many well owners permitted the measurement of their wells, and town officiais and residents supplied valuable information. Luther Wilkens, manager of the office of the Rural Electrification Administration in Akron, Colo., supplied information that greatly facilitated the investigation, and Byrle Milier and associates of the REA, office in Holyoke, Colo., were cooperative and helpful. W. E. Code of the Colorado Agricultural Experiment-Station at Fort Collins supplied many data that he had previously collected. Drilling contractors supplied well logs and other data; the Chicago, Burlington \& Quincy Railroad Co. supplied logs of its wells and test họles; and oil companies supplied logs of seismograph shot holes and the altitude of the top of the Pierre shale in many of the shot holes and the altitude of the land surface at others.

\section{GEOGRAPHY}

Topography and drainage

The investigated region lies entirely within the High Plains section of the Great Plains physiographic province. South of the Arikaree River the region is typically "short grass" country consisting of broad, flat areas, which in places grade into gently rolling upland divides that are dissected by narrow, shallow stream valleys. This area is dotted by undrained saucerlike depressions, which range in diameter from a few feet to a few thousands of feet. Their origin has been variously explained by Darton and others (1915, p. 36-37), Johnson (1901, p. 702-712), Hay (1895, p. 555556), and Judson (1950, p. 253-273). Locally, the major streams have cut narrow, shallow canyons through the Tertiary strata into the underlying Pierre shale.

North of the Arikaree River the region is mantled extensively by dune sand. In general, the dune-sand area has a multicycle dune 
topography as described by Smith (1940, p. 164). In places of rejuvenation, secondary blowouts are common. Dunes in these areas are comparatively bare and probably are migrating to some extent. Elsewhere the dunes are in the youth to maturity stages of the eluvial phase of the sand-dune cycle and are protected by a vegetal cover sufficient to support numerous cattle. According to Wallace Bruce of the U. S. Conservation Service, soil in the dune-sand area ranges in thickness from a featheredge on the side slopes of the dunes to as much as 3 feet in the valleys between the dunes. Some of these interdune valleys are under cultivation. The interior drainage, which is typical of the eluvial phase of the sand-dune cycle, combined with the permeability of the dunes enables precipitation to percolate rapidly downward to the water table; losses by evaporation, transpiration, and runoff thus are reduced.

The entire upland plain slopes gently from west to east. The altitude of the land surface ranges from more than 5,000 feet above sea level in the west to slightly less than 4,000 feet in the east.

Ladder Creek, Smoky Hill River, North Fork of the Smoky Hill River, Landsman Creek, Spring Creek, South Fork of the Republican River, Arikaree River, North Fork of the Republican River, Red Willow Creek, Sandy Creek, Patent Creek, and Frenchman Creek head in the western part of the region and flow southeastward or eastward. Most of the streams flow only after relatively heavy rains; however, Landsman Creek, both forks of the Republican River, and the Arikaree River are perennial in their lower reaches where they have become effluent or gaining streams by cutting downward until their channels are below the water table. (See fig. 3.) Ground water moves along the eroded surface of the relatively impermeable Pierre shale and is discharged into these streams largely by seeps and springs. Flood waters in the intermittent water courses probably provide considerable recharge to the ground-water reservoir from loss of flow by percolation through the stream bed; streams of this type are called losing or influent streams. (See fig. 3.)
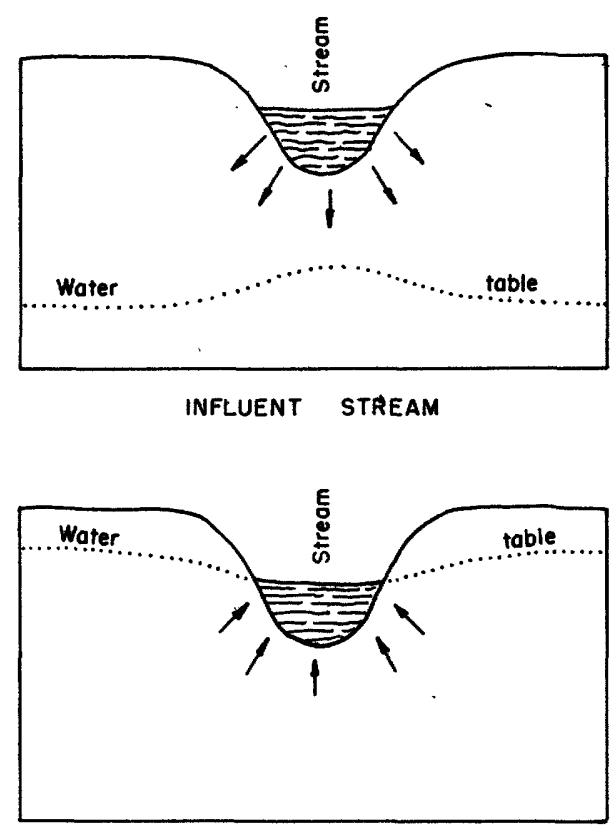

EFFLUENT STREAM

Figure 3.--Diagrammatic sections showing the relationship of a stream to the water table.

\section{Climate}

The climate is the typically semiarid type of the High Plains and is characterized by little precipitation, high evaporation, low humidity, and much sunshine and wind. The summer days are hot and the nights are cool. The winters are relatively mild with occasional short periods of severe cold. The region is subject to blizzards in the fall and spring. Average annual temperatures at the stations of the United States Weather Bureau in the area are as follows: Akron, 48.50F; Burlington, 49.20 F Holyoke, 49.20 F Jules burg, 49.5\% ; Kit Carson, 5l.10F; Stratton, 51.60F; Wray, 50.80F; and Yuma, 49.00F. The precipitation is greatest during the late spring and summer and is least during the win. ter. Precipitation graphs prepared from U. S. Weather Bureau records are shown in figure 4. 


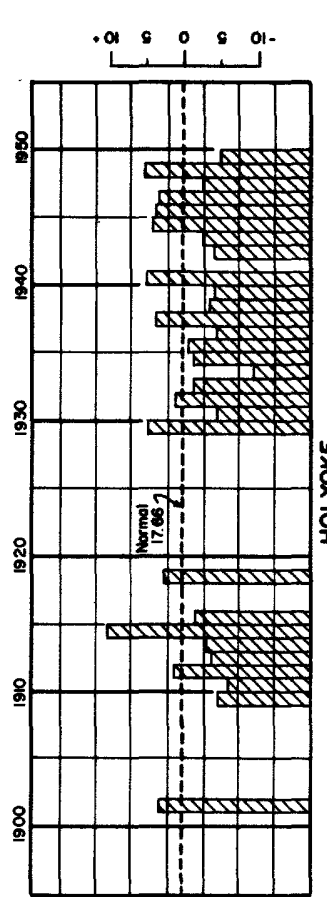

品\& \& 8.0

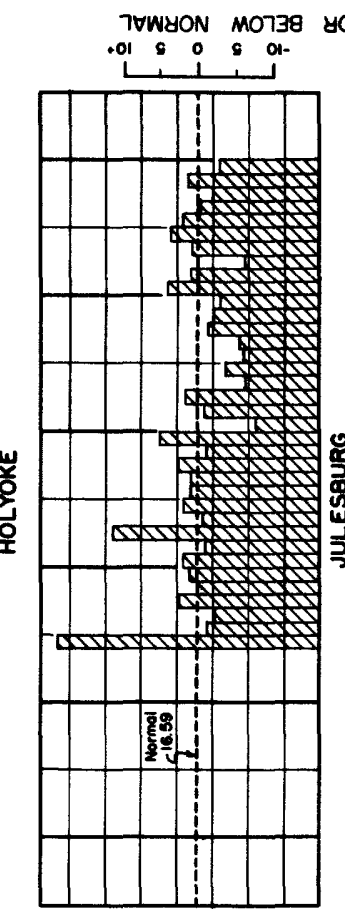

$88 \% 8 \div 0$

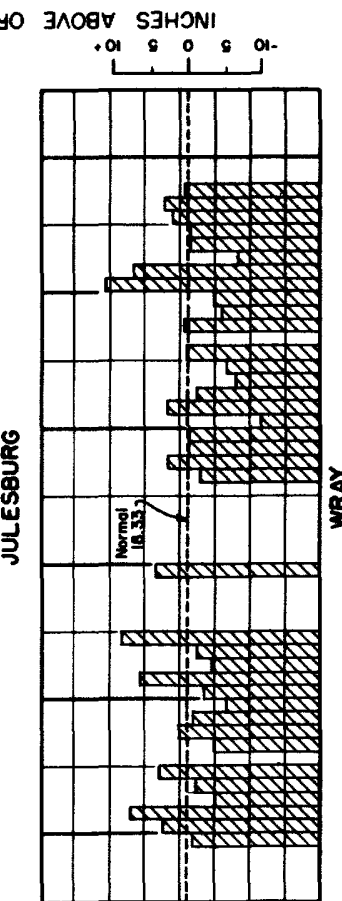

$\circ \%$ 只

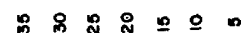

inOIIVIIdIJARd

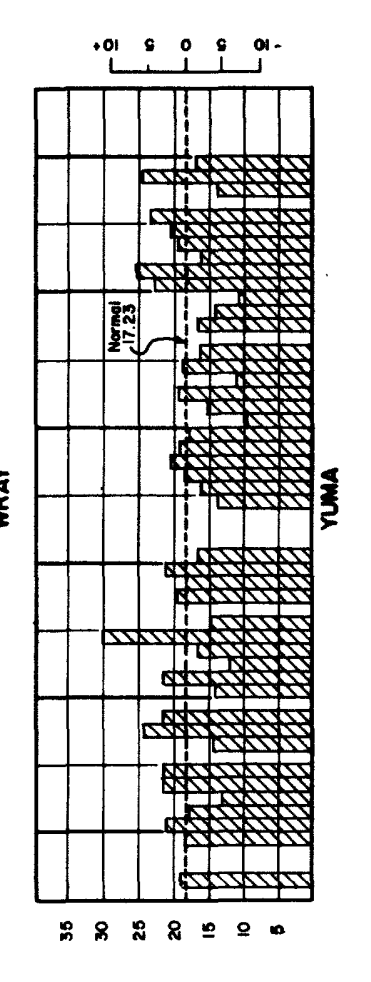

栾

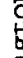

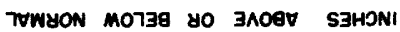
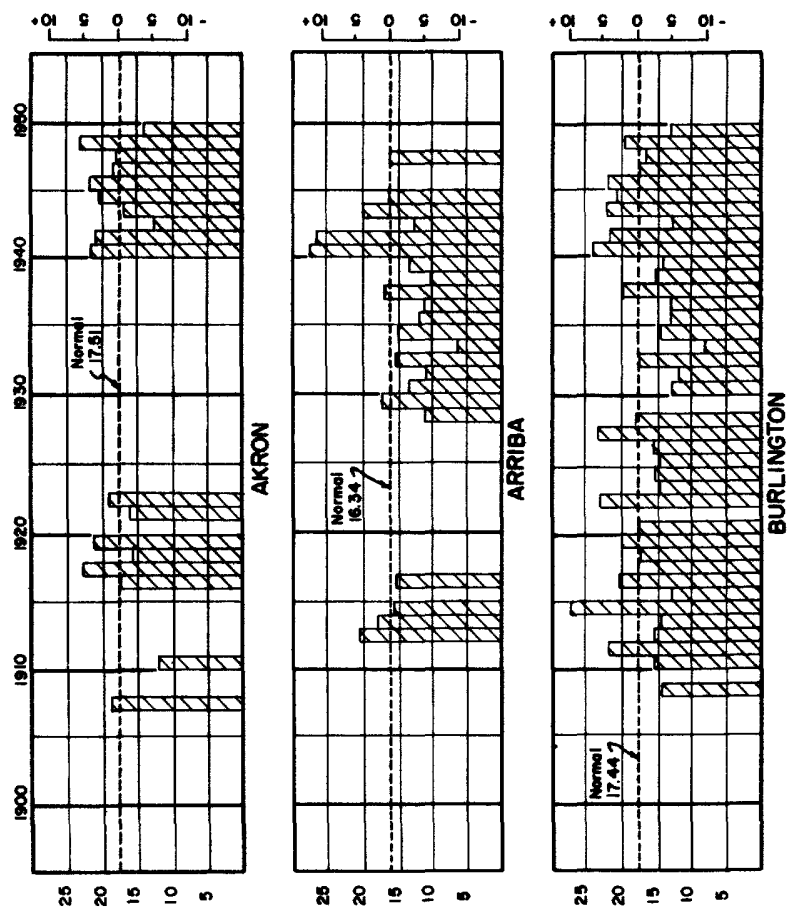

$.01 \leq 0 \leq a$
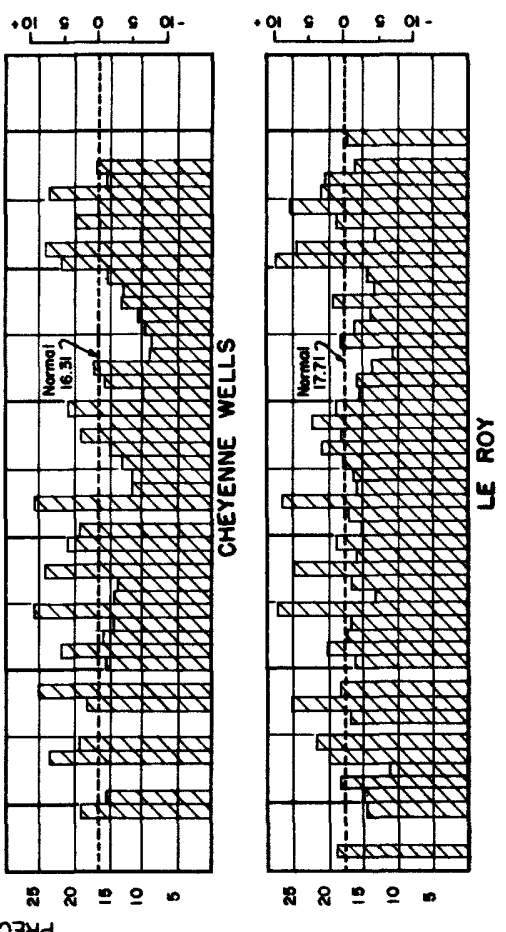

요으. 
GEOLOGY

Stratigraphy

The exposed sedimentary rocks in the region range in age from Late Cretaceous to Recent. The oldest formation is the Pierre shale which underlies the entire region and which crops out in the deeper valleys.

Most of the area from Wray northward to the South Platte valley is shown on the State Geologic map to be underlain by the Arikaree sandstone of Tertiary (Miocene) age. Available data indicate, however, that very little of the area is underlain by the Arikaree. Detailed mapping in the Wray area (Hill and Tompkin, 1953) has shown that the rocks previously mapped as Arikaree in that area are in large part the Ogallala formation. Hydrologic data obtained as a part of this investigation indicate that the Ogallala formation underlies most of the area previously mapped as the Arikaree sandstone. Wells throughout the area have specific capacities much greater than those of wells commonly found in areas underlain by the Arikaree. If the Arikaree underlies any of the Kansas River basin in Colorado, it is considered a part of the Ogallala formation for the purpose of this report.

The Ogallala formation crops out in many places in the area and ranges in thickness from a featheredge to several hundred feet. It is reported by Hill and Tompkin (1953) to be overlain locally by the Grand Island formation of Pleistocene age (here considered a part of the Ogallala), and in the eastern and southern parts of the area it is overlain by the Sanborn formation of Pleistocene and Recent(?) age. Dune sand, which mantles large areas in the northern part of the region, probably is of Pleistocene to Recent age. Alluvium of Recent age underlies the channels and flood plains of some of the streams.

The geology of the region was not mapped during the investigation. Surficial materials are not identified by stratigraphic terms in the logs (table 2) except where the drillers' logs indicate accurate lithologic subdivisions.
Geologic formations and their water-bearing properties

Cretaceous system

Pierre shale (Upper Cretaceous). --The Pierre shale underlies the entire region and crops out in the eastern lower reaches of the valleys of the Arikaree and North Fork of the Republican River, in bluffs at Flagler and Genoa, and at a few other places where the streams have cut through the mantle of younger materials.

Weathered outcrops of the Pierre shale are light to dark gray and fresh exposures are generally dark gray, blue, or black. The Pierre contains numerous concretions of limonite and calcium carbonate. The shale is overlain in many areas by a dense yellow clay (sometimes called soapstone by drillers and local residents) ranging in thickness from a featheredge to several feet. This clay probably was formed by weathering of the Pierre shale in early Tertiary time.

The Pierre shale is relatively impermeable and yields water only sparingly to wells from fractured zones, openings along bedding planes, or thin beds of sand within the formation. The water generally is of poor quality and is not available in quantities sufficient for irrigation.

The Pierre shale ranges in thickness from about 600 feet in Wallace County, Kans., to about 1,400 feet in northwestern Cheyenne County, Kans., and thickens westward to about 1,490 feet in T. 2 S., R. 43 W., Yuma County, Colo., and to about 2,560 feet in T. 1 S., R. 49 w., Washington County, Colo.

Regionally, the eroded surface of the Pierre shale appears to be relatively smooth (see pl. 2); locally, however, the Tertiary streams probably eroded channels into the Pierre shale. A map showing the configuration of the trosion surface on the Pierre shale in parts of Cheyenne, Kit Carson, and Lincoln Counties (pl. 2) was prepared from logs of seismograph shot holes. The over-all erosion surface is smooth except where the Ogallala cover has 
been removed, as in the valley of Big Sandy Creek. Its smoothness is further illustrated by the diagrammatic cross section (pl. 3) which indicates that the erosion surface of the Pierre shale slopes north-northeastward at a rate of approximately 33 feet per mile.

\section{Tertiary system}

Ogallala formation (Pliocene).--The Ogallala formation is composed of silt, sand, gravel, caliche, and clay which appear, on cursory examination, to occur in lenses that interfinger in short vertical and horizontal distances. The sorting and continuity of the beds actually are better developed than they appear surficially to be (Fenneman, 1931, p. 13). In general, gravel and sand are concentrated in winding narrow bands extending in an easterly direction. The presence of these bands at any depth in the formation suggests that they represent former channels of the shifting streams that deposited the Ogallala formation. Silt, however, is the predominant material throughout the formation. The local concentration of sand and gravel was probably caused by more active stream erosion, which resulted in the washing away of the finer constituents and in leaving of the sand and gravel as residual material (Fenneman, 1931, p. 13).

The sand and gravel of the Ogallala formation is cemented in places by calcium carbonate and forms a fairly well indurated rock; in other places, it is well cemented into hard, resistant ledge-forming beds (the socalled mortar beds). Stringers of clay and caliche are present almost throughout the formation. Bentonitic clay, which occurs in the lower half of the Ogallala formation in some areas and which was described by Elias (1931, p. 153-158), is possibly present in eastern Colorado. A hard, opalescent, porous rock containing grains of sand crops out in the area south of the Arikaree River. I have found in eastern Colorado no outcrops of indurated volcanic ash similar to those described by Elias (1931, p. 211).

One of the most widespread horizons is the capping "algal limestone," a reddish-brown, concentrically banded limestone that weathers to a miniature badlands surface. Elias (1931, p. 136-141) believes that this limestone was precipitated in part by the alga Chlorellopsis. The thickness of the bed is remarkably uniform in all studied exposures; it ranges from 2 to $3 \frac{1}{2}$ feet.

The Ogallala formation yields water to wells throughout the region except where it thins to a featheredge. The water generally is of good quality and is suitable for stock, irrigation, domestic, and public supply. All towns in the region except Wray utilize water from the Ogallala formation for public water supply. The formation yields water to wells in sufficient quantity for irrigation in many areas, especially where wells tap saturated sand and gravel filling the channels of old Tertiary streams. Extensive test drilling to locate a possible buried channel is recommended before a well site is chosen. Calcium carbonate cement or clay, silt, and fine sand may reduce the permeability of the Ogallala in some areas to the extent that water in sufficient quantities for irrigation cannot be obtained.

The lithology of the Ogallala formation is graphically shown in the diagrammatic cross section of part of Kit Carson County, Colo. (pl. 3), which was prepared from the drillers' logs of seismograph shot holes. The position of the water table in the diagram is based on measured water levels and on water levels reported by residents in the area. The cross section shows that the depth to water, as well as the saturated thickness, determines the possible extent of irrigation-well development. Some of the southeastern part of the area shown in the diagrammatic cross section is underlain by the Sanborn formation, but the depth to the contact between the Sanborn and Ogallala formations could not be determined in the drillers' logs.

Quaternary system

Sanborn formation (Pleistocene).--The Sanborn formation of Pleistocene and Recent(?) age consists mainly of yellow-buff porous unstratified and unconsolidated loess. The 
Ogallala is overlain locally by this formation. Farther east, in Wallace, Sherman, and Cheyenne Counties, Kans., the formation is thicker and is much more widespread. The Sanborn formation lies above the water table in this region and, hence, does not yield water to wells.

Dune sand (Pleistocene to Recent). - The dune sand of Pleistocene to Recent age in the northern part of the region consists largely of reworked material derived from the Ogallala formation. The dune sand yields water to stock wells where the water table is close to the surface. The hydrologic importance of the dune-sand area, however, is due to its ability to absorb water rapidly. Because of the high porosity and permeability of the dune sand and because of the poorly developed surface drainage, much of the water from precipitation percolates downward to the zone of saturation and little water is lost by runoff, evaporation, or transpiration.

Alluvium (Recent).--Alluvium of Recent age, consisting of gravel, sand, silt, and clay, underlies the flood plains of the major streams. The alluvium yields moderate amounts of water to stock and domestic wells and supplies irrigation wells in the Arikaree Valley near Cope. Alluvium of the North Fork of the Republican River yields water to domestic and stock wells and to the municipal wells at Wray.

USE OF GROUND WATER

\section{Development of irrigation}

Irrigation is a comparatively new undertaking for most of the ranchers and farmers in eastern Colorado. Since 1947 the number of irrigation wells in the region has more than trebled, and the number of newly constructed irrigation wells is increasing markedly each year (see fig. 5). The drillers of the region are busy with additional installations at the present time (April 1951). Although mistakes have been made, well owners are rapidly improving their irrigation methods and more efficient pumping plants are being

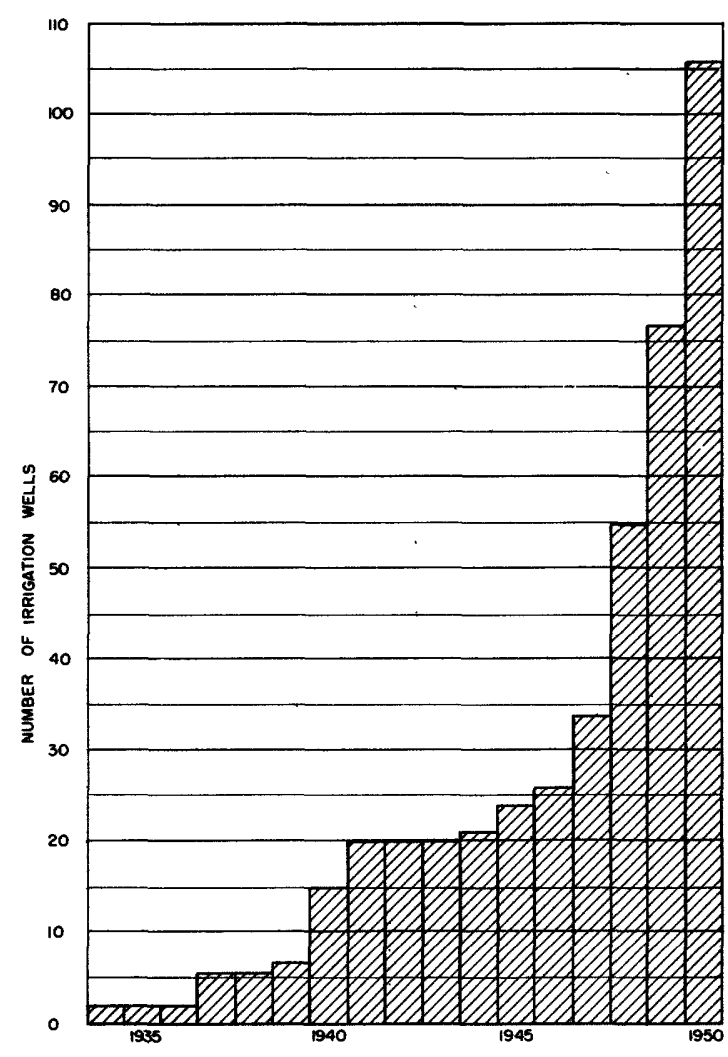

Figure 5.--Cumulative number of irrigation wells. Wells constructed before 1934 and now used for irrigation are not included because they were intended originally to be used for other purposes. Wells for which the year of construction could not be determined also are not included.

installed. Crops that can be irrigated most profitably are receiving more attention, and many owners keep careful records of the cost of fuel for pumping, the fuel consumption, and the production increase. Alfalfa is probably the principal irrigated crop and various row crops are next in importance. Pasture and wheat also are irrigated in some places.

\section{Summary of well data}

Data compiled for wells in the region (see table 1) are summarized on the following page. 
Use of water from inventoried wells

\begin{tabular}{|c|c|c|c|c|c|c|}
\hline \multirow{3}{*}{ County } & \multicolumn{6}{|c|}{ Number of wells for indicated use } \\
\hline & \multicolumn{2}{|c|}{ Irrigation } & \multirow{2}{*}{$\begin{array}{l}\text { Public } \\
\text { supply }\end{array}$} & \multirow{2}{*}{$\begin{array}{l}\text { Water-level } \\
\text { observation }\end{array}$} & \multirow[b]{2}{*}{ Stock } & \multirow[b]{2}{*}{ Cemetery } \\
\hline & Now in use & $\begin{array}{l}\text { Additional } \\
\text { to be used }\end{array}$ & & & & \\
\hline 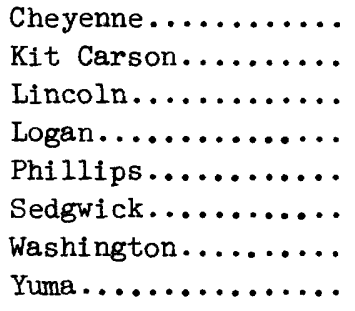 & $\begin{array}{r}2 \\
16 \\
5 \\
1 \\
7 \\
1 \\
36 \\
22\end{array}$ & $\begin{array}{r}2 \\
12 \\
0 \\
0 \\
1 \\
0 \\
14 \\
2\end{array}$ & $\begin{array}{r}3 \\
15 \\
2 \\
3 \\
4 \\
0 \\
6 \\
8\end{array}$ & $\begin{array}{l}1 \\
3 \\
0 \\
2 \\
2 \\
0 \\
4 \\
0\end{array}$ & $\begin{array}{l}0 \\
1 \\
0 \\
0 \\
1 \\
0 \\
0 \\
1\end{array}$ & $\begin{array}{l}0 \\
1 \\
0 \\
0 \\
0 \\
0 \\
0 \\
0\end{array}$ \\
\hline & 90 & 31 & 41 & 12 & 3 & 1 \\
\hline
\end{tabular}

Yield and specific capacity of irrigation wells

$[E$, estimated or reported; $M$, measured]

\begin{tabular}{|c|c|c|c|c|c|c|}
\hline \multirow[b]{2}{*}{ County } & \multicolumn{3}{|c|}{ Yield } & \multicolumn{3}{|c|}{ Specific capacity } \\
\hline & $\begin{array}{c}\text { Number of } \\
\text { wells }\end{array}$ & $\begin{array}{l}\text { Range } \\
\text { (gpm) }\end{array}$ & $\begin{array}{c}\text { Average } \\
\text { (gpm) }\end{array}$ & $\begin{array}{c}\text { Number of } \\
\text { wells }\end{array}$ & $\begin{array}{l}\text { Range } \\
\text { (gpm/ft) }\end{array}$ & $\begin{array}{l}\text { Average } \\
(\mathrm{gpm} / \mathrm{ft})\end{array}$ \\
\hline 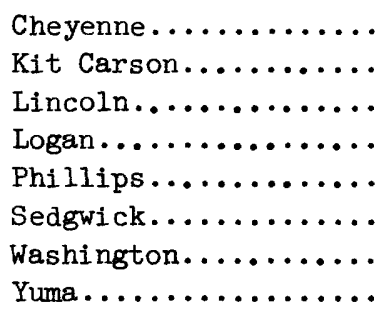 & $\begin{array}{r}4 \\
22 \\
5 \\
1 \\
7 \\
1 \\
40 \\
22\end{array}$ & $\begin{array}{c}100 \mathrm{E}-400 \mathrm{E} \\
125 \mathrm{E}-1,600 \mathrm{E} \\
241 \mathrm{M}-1,000 \mathrm{E} \\
600 \mathrm{E} \\
485 \mathrm{M}-1,500 \mathrm{E} \\
660 \mathrm{E} \\
100 \mathrm{E}-1,213 \mathrm{M} \\
110 \mathrm{E}-1,650 \mathrm{E}\end{array}$ & $\begin{array}{r}175 \\
700 \\
468 \\
600 \\
1,114 \\
660 \\
630 \\
823\end{array}$ & $\begin{array}{r}1 \\
10 \\
0 \\
1 \\
7 \\
1 \\
9 \\
20\end{array}$ & $\begin{array}{l}20 \\
20-125 \\
\ldots \ldots \\
100 \\
9-123 \\
26 \\
32-243 \\
10-116\end{array}$ & $\begin{array}{r}20 \\
41 \\
\cdots \\
100 \\
85 \\
26 \\
79 \\
44\end{array}$ \\
\hline & 102 & $100 E-1,650 E$ & 697 & 49 & $10-243$ & 56 \\
\hline
\end{tabular}

Depth of irrigation wells

$[R$, reported; $M$, measured $]$

\begin{tabular}{|c|c|c|c|}
\hline County & $\begin{array}{c}\text { Number of } \\
\text { wells }\end{array}$ & $\begin{array}{l}\text { Range } \\
\text { (feet) }\end{array}$ & $\begin{array}{r}\text { Average } \\
\text { (feet) }\end{array}$ \\
\hline \multirow[t]{2}{*}{ 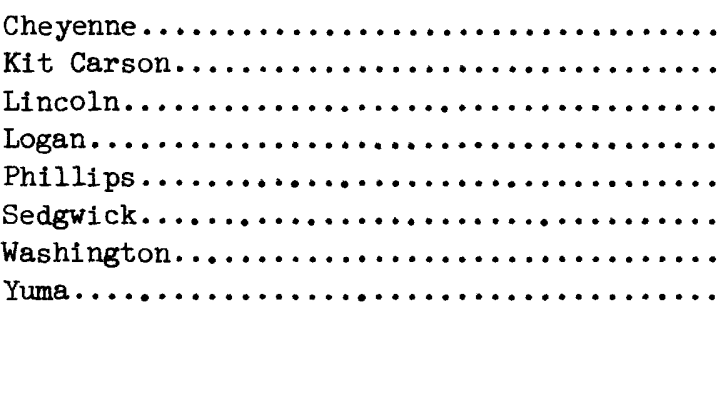 } & $\begin{array}{r}4 \\
24 \\
5 \\
1 \\
7 \\
1 \\
45 \\
23\end{array}$ & $\begin{array}{c}13.6 \mathrm{M}-72.3 \mathrm{M} \\
17.5 \mathrm{M}-308 \mathrm{R} \\
110 \mathrm{R}-260 \mathrm{R} \\
78 \mathrm{R} \\
198 \mathrm{R}-272 \mathrm{R} \\
320 \mathrm{R} \\
13.5 \mathrm{M}-76.8 \mathrm{M} \\
28.0 \mathrm{M}-326 \mathrm{R}\end{array}$ & $\begin{array}{r}45 \\
111 \\
148 \\
78 \\
225 \\
320 \\
47 \\
154\end{array}$ \\
\hline & 110 & $13 \cdot 5 M-326 R$ & 101 \\
\hline
\end{tabular}


Area irrigated in 1950 and prospective irrigated area

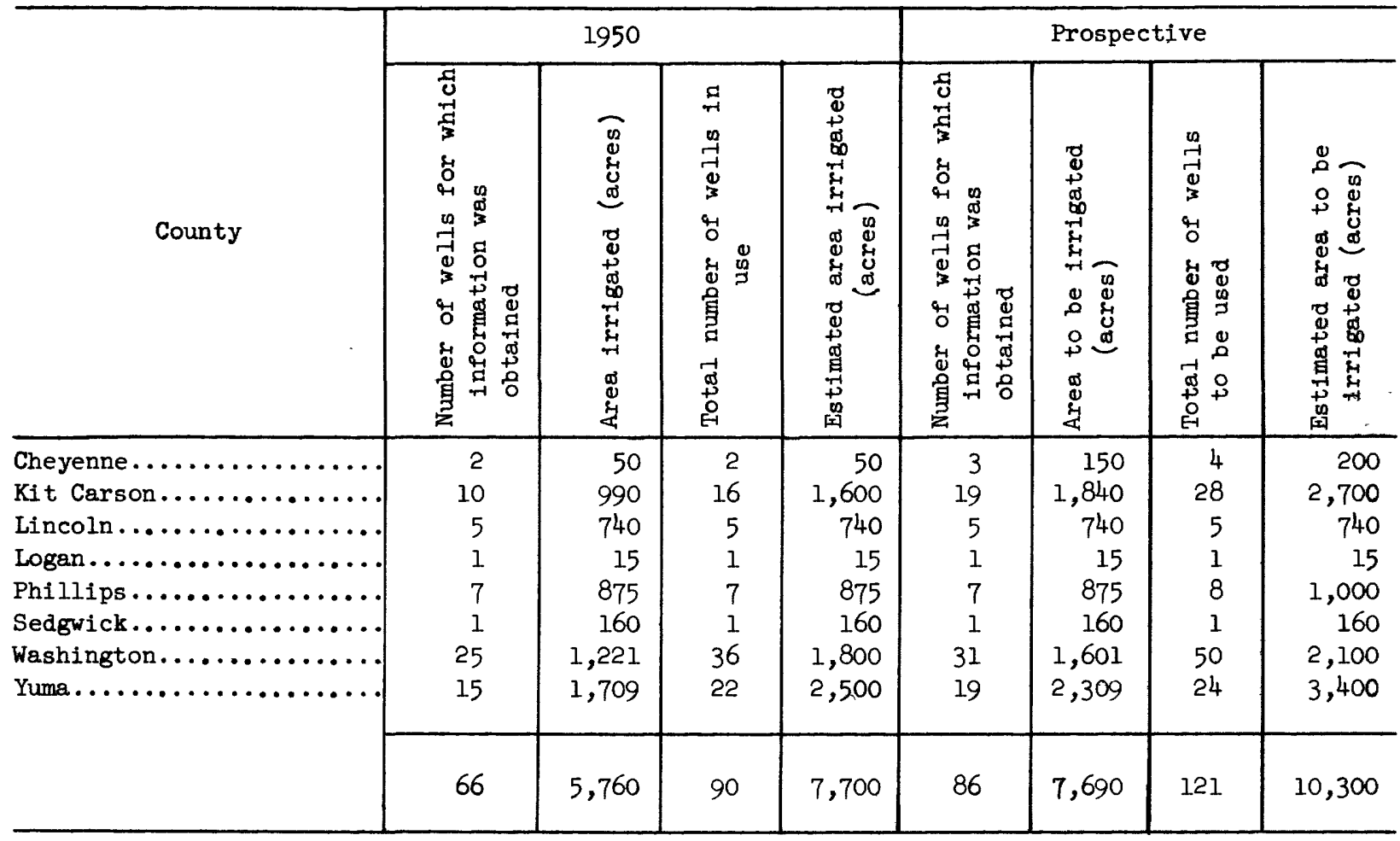

Depth to water below land surface, drawdown, and pumping lift of irrigation wells $[R$, reported; $M$, measured]

\begin{tabular}{|c|c|c|c|c|c|c|c|c|c|}
\hline \multirow{2}{*}{ County } & \multicolumn{3}{|c|}{$\begin{array}{l}\text { Depth to water below } \\
\text { land surface }\end{array}$} & \multicolumn{3}{|c|}{ Drawdown } & \multicolumn{3}{|c|}{ Pumping lift } \\
\hline & $\begin{array}{c}\text { Number } \\
\text { of wells }\end{array}$ & $\begin{array}{l}\text { Range } \\
\text { (feet) }\end{array}$ & $\begin{array}{r}\text { Average } \\
\text { (feet) }\end{array}$ & $\begin{array}{c}\text { Number } \\
\text { of 'wells }\end{array}$ & $\begin{array}{l}\text { Range } \\
\text { (feet) }\end{array}$ & $\begin{array}{r}\text { Average } \\
\text { (feet) }\end{array}$ & \begin{tabular}{|c|} 
Number \\
of wells
\end{tabular} & $\begin{array}{l}\text { Range } \\
\text { (feet) }\end{array}$ & $\begin{array}{r}\text { Average } \\
\text { (feet) }\end{array}$ \\
\hline Cheyenne.... & 4 & $8.3 M-29 R$ & 20 & 1 & 5 & 5 & \begin{tabular}{l|}
1 \\
\end{tabular} & 13 & 13 \\
\hline Kit Carson. . & 26 & $5.0 \mathrm{M}-191.8 \mathrm{M}$ & 58 & 10 & $5-58$ & 28 & 10 & $16-206$ & 83 \\
\hline Lincoln.... & 5 & $60 R-200 R$ & 94 & 0 & $\ldots \ldots \ldots$ & .. & 0 & $\ldots \ldots \ldots$ & $\ldots$ \\
\hline Logan . . . . . . & 1 & $52.6 \mathrm{M}$ & 52.6 & 1 & 6 & 6 & 1 & 59 & 59 \\
\hline Phillips.... & 7 & $39.9 M-148.2 M$ & 76 & 7 & $10-53$ & 18 & 7 & $53-201$ & 94 \\
\hline Sedgwick.... & 1 & $202.5 \mathrm{M}$ & 202.5 & 1 & 25 & 25 & 1 & 227 & 227 \\
\hline Washington. & 46 & $9.8 M-47.9 M$ & 27 & 9 & $5-28$ & 16 & 9 & $28-61$ & 41 \\
\hline \multirow[t]{2}{*}{ Yuma......... } & 24 & $6.6 \mathrm{M}-230.2 \mathrm{M}$ & 73 & 20 & $7.5-60$ & 27 & 20 & $19-251$ & 102 \\
\hline & 114 & $5.0 \mathrm{M}-230.2 \mathrm{M}$ & 51 & 49 & $5-60$ & 23 & 49 & $13-251$ & 86 \\
\hline
\end{tabular}


Possibilities for development of irrigation from wells

Wells of large yield situated between areas where irrigation wells locally are numerous indicate that irrigation from wells probably could be developed throughout broad areas at the present time. Irrigation from wells also may be feasible in the future in other areas where the total lift now is too great for economical development of the ground-water resources. These marginal areas, however, might reasonably be opened to extensive pump irrigation at an earlier date if a cheap fuel, such as natural gas, became available. Of interest in this regard is the recent leasing by oil producers of large areas in northeastern Colorado.

Irrigation development in large areas in the region cannot be condemned or endorsed categorically without detailed ground-water studies. The lenticular character and the wide range in the permeability of the waterbearing beds in the Ogallala formation and the narrowness of the underlying Tertiary stream channels make it possible to obtain wells of high yields close to those of much lower yields. For these reasons, local lines of test holes should be drilled before the site of an irrigation well is selected. Inasmuch as the braided Tertiary streams that laid down the sand and gravel of the Ogallala formation generally flowed from the Rocky Mountains in the same direction as the present-day streams, it seems advisable to test drill as nearly as possible at right angles to their courses--that is, in north-south or northeast-southwest lines--in order to locate the more permeable water-bearing materials.

Additional irrigation wells almost certainly will be drilled southeast of Holyoke in the Frenchman Creek basin. (See pl. 1.) The water table is comparatively shallow and the average yield of all the pumped wells is more than 1,000 gpm. The depth to the water table gradually increases from approximately 50 feet in the Frenchman Creek valley to more than 200 feet in southern Sedgwick County; therefore, the water table in the area south of Amherst probably is about 125 feet below the surface. The high average specific capacity of wells in Phillips County indicates that pumping water for irrigation probably would be profitable in that area. (See table 1.) Irrigation by flooding methods is feasible in this area as the land is relatively level and the soil is less sandy than elsewhere in the region.

The yield of well B6-50-14da in Logan County is reported to be $600 \mathrm{gpm}$ and the drawdown is reported to be 6 feet; if these figures are correct, then the specific capacity of this well is $100 \mathrm{gpm}$ per foot of drawdown. The static water level in this well is about 53 feet below the land surface. Little information has been obtained for the area between this well and those southeast of Holyoke, but conditions for using water from wells for irrigation can be assumed to be reasonably favorable in parts of this area.

Irrigation by sprinkling probably will increase slowly in the area of dune sand south of Holyoke and north of Wray, particularly in areas of mature dunes where soils are more completely developed. The water table is shallow and the aquifer is moderately permeable, as indicated by data for well B5-43$24 \mathrm{ab}$, which reportedly yields $1,650 \mathrm{gpm}$ with a drawdown of 19 feet.

From the dune-sand area westward to the limits of the Kansas River basin, the increase of irrigation probably will be slow. Much of the area is sandy and irrigation by sprinkler systems generally would be required. The water table in this area is reported to be deep.

More irrigation may be done in the valley of the North Fork of the Republican River both east and west of Wray if both surface water from the river and ground water from the alluvium and from the Ogallala formation are utilized. Well B2-42-2cc east of Wray yields $750 \mathrm{gpm}$ with a drawdown of 18 feet; there, however, the Pierre shale lies at a depth of only 65 feet.

Very little irrigating has occurred in the large area that extends southward from U. S. Highway 34 to the Arikaree Valley. Inasmuch as the area is sandy, future development, if any, will be slow and of necessity mostly by sprinkler systems. There also the water table is reported to be generally deep. 
The greatest use of wells for irrigation has been in the Arikaree valley in the vicinity of Cope. More wells utilizing ground water from both the alluvium of the Arikaree River and from the Ogallala formation could be developed there. In the western part of the Arikaree River basin north of Genoa, Bovina, and Arriba, irrigation by ground water from the alluvium may be developed. Although the water is considered hard by the local residents, it probably is suitable for irrigation. The water table on the tributary divides, however, is deep, and it is doubtful whether water from the Ogallala formation will be used for irrigation in that area.

From Cope northwestward to Anton, farmers have difficulty in obtaining sufficient water for stock wells. The top of the Pierre shale is reported to be near the surface and the zone of saturation above it, where present, is very thin.

East of Cope, near Joes and Kirk, the water table is about 100 feet below the surface, and the yield from wells in the area ranges from $344 \mathrm{gpm}$ to $1,350 \mathrm{gpm}$. The average yield is $726 \mathrm{gpm}$, and the average drawdown is 24 feet. Many irrigation wells probably will be developed in this area. Farther east, north of Idalia, the water table is more than 200 feet below the land surface; however, the large yield of the wells and the good soil have encouraged the construction of a few irrigation wells.

Northeast of Cope along the Arikaree valley to the Kansas State line, irrigation from wells possibly could be increased extensively. The Ogallala formation yields water to wells at shallow depths in this area, and, where the Ogallala is thin, water for irrigation probably could be obtained from wells in the alluvium.

Irrigation wells are now widely scattered along the South Fork of the Republican River. More wells probably could be drilled between the existing ones and could be pumped without excessive lowering of water levels.

The conditions for development of irrigation from wells in the Landsman Creek valley are similar to those in the Arikaree valley northeast of Cope.
More irrigation wells could be installed in the area of the North Fork of the Smoky Hill River southeast of Burlington, as well as in the area south of Burlington in the basins of Sand and Beaver Creeks. The depth to water in an observation well $0.5 \mathrm{mile}$ west of Burlington is 170 feet; it is about 120 feet in the valley of Beaver Creek and about 90 feet in the valley of Sand Creek. (See pl. 3.) The water table is shallow in T. $10 \mathrm{S.}$, Rs. 42 and $43 \mathrm{~W}$. , and in the southern part of R. $44 \mathrm{~W} .$, and for this reason development of ground water for irrigation in these areas is very likely. Likewise, T. $9 \mathrm{~S} ., \mathrm{R} .42 \mathrm{~W}$. appears to be a favorable area for development of irrigation. The depth to the water table is progressively greater from R. $44 \mathrm{~W}$. to R. $47 \mathrm{~W}$. About the only other area where the water table is shallow is in the valley of Spring Creek near Vona.

Farther south from Burlington, the development of irrigation probably will be slow and will be restricted mainly to irrigation from wells in the alluvium of the South Fork of the Smoky Hill River and its tributaries. The depth to water below the topographic divides in this area appears to be too great for the economical development of the ground water for irrigation at the present time. Little information about ground water was obtained in large areas in the region because field data were collected only in those areas where some irrigation by pumping has already begun. Possibly some of these relatively unexplored areas may ultimately be developed.

Widespread irrigation is unlikely in the extensive areas of dune sand because the soil is poorly developed and the slopes are steep. Irrigation is unlikely also near the periphery of the outcrop of the Ogallala formation because the zone of saturation in the formation is too thin.

Municipal water supplies

Sixteen municipalities in the region obtain their public water supplies from wells. All except Wray, which obtains water from the alluvium or terrace deposits (or both) of the 
North Fork of the Republican River, obtain water from the Ogallala formation.

Akron

Akron is supplied by 5 wells (B2-52-17cb, $-17 c c, 3-52-8 a a,-8 b d$, and $-17 d a)$ that range in depth from 41.5 to 170 feet; the static water level in these wells ranges in depth from 13.6 to 110 feet, and the yields range from 35 to $1,000 \mathrm{gpm}$. All the wells are equipped with electrically driven turbine pumps. A ground-level reservoir at the northwest edge of town has a capacity of $1,000,000$ gallons, and an elevated tank on the highway 1 block east of Main Street has a capacity of 100,000 gallons. Water is pumped to the reservoirs and mains by the well pumps. The water is chlorinated and an estimated 260,000 gallons is consumed daily.

Arriba

Arriba is supplied from 2 wells, C9-5312aal and -12a.2, which are equipped with electrically driven turbine pumps. The wells are about 85 feet deep and the depth to water is about 70 feet. Well C9-53-12aal pumps about $39 \mathrm{gpm}$ and well C9-53-12aa2 pumps about $49 \mathrm{gpm}$. The pneumatic reservoir has a capacity of 18,000 gallons. An estimated 23,600 gallons of water is consumed daily.

Bethune

Bethune is supplied from 1 well, c8-45$34 \mathrm{cc}$, which is equipped with an electrically driven turbine pump. The well is 230 feet deep, the depth to water is 162 feet, and the yield is reported to be $125 \mathrm{gpm}$. Storage is provided by an 11,500-gallon elevated tank. The water is pumped directly into the mains. The average daily consumption is reported to be 3,300 gallons.
Burlington

Burlington is supplied from 3 wells: C844-36cd at the corner of 15 th Street and U. S. Highway 24, C8-44-36ab at the corner of $14 \mathrm{th}$ and Railroad Streets, and C8-44-36dd at the corner of 10th Street and Lowell Avenue. All wells are equipped with electrically driven turbine pumps. The wells range in depth from 230 to 350 feet and the depth to water in all wells is reported to be 150 feet. The yield of the wells ranges from 300 to $500 \mathrm{gpm}$. Storage is provided by a 50,000-gallon elevated tank on the north side of town. All wells pump directly into the mains. The average daily consumption is reported to be 300,000 gallons.

Cheyenne Wells

Cheyenne Wells is supplied from 3 wells (cl4-44-20dcl, -20dc2, and -20dc3) in the southern part of town. Each well is equipped with an electrically driven turbine pump. The wells range in depth from 265 to 365 feet and the static water level is about 250 feet below land surface. The yield of well c1444-20dcl is not known; the yield of wells C14-44-20dc2 and $-20 d c 3$ are reported to be $300 \mathrm{gpm}$ and $200 \mathrm{gpm}$, respectively. An elevated tank northwest of town provides storage for 85,000 gallons. Although the water apparently is of good quality, chlorine is used for precautionary sanitation. Sand, which sometimes is pumped with the water, damages the water meters. The average daily water consumption is reported to be 264,000 gallons.

Eckley

Eckley is supplied from 1 well (B2-46$26 \mathrm{bc}$ ) which is 317 feet deep, in which the static water level is 45 feet below the land surface and the yield is $200 \mathrm{gpm}$. The well is equipped with an electrically driven turbine 
pump. Storage is provided by an elevated tank that has a capacity of 50,000 gallons. Water is pumped directly into the mains. The average daily consumption is 20,000 gallons.

\section{Flagler}

Flagler is supplied from 2 wells (C9-51$2 a c$ and -2bd) that are equipped with electrically driven turbine pumps. The depth of the wells is about 100 feet. The water level in well C9-5l-2ac is about 92 feet and the water level in the other well is about 63 feet below the land surface. Well c9-5l-2ac is reported to yield about $100 \mathrm{gpm}$ and well C9-51-2bd about 220 gpm. A 100,000-gallon elevated tank near the high school is used for storage. The water is pumped directly into the mains. The average daily consumption is estimated to be 83,000 gallons.

Fleming

Fleming is supplied by 3 wells. Well B849-9adl at the elevated storage tank is 165 feet deep, is equipped with an electrically powered cylinder pump, and yields approximately $12 \mathrm{gpm}$. It is used only in the summer during periods of water shortage. Well B849-9ad2 is 200 feet deep and the static water level is about 125 feet below land surface. This well is equipped with an electrically driven turbine pump and yields $22 \mathrm{gpm}$. Well $\mathrm{B} 8-49-10 \mathrm{bb}$ is also 200 feet deep, has an electrically driven turbine pump, and yields $50 \mathrm{gpm}$. Storage is provided by a 50,000gallon elevated tank west of town. The water is pumped directly into the mains. A reported 33,000 gallons is consumed daily.

Genoa

Genoa is supplied by springs that issue at the contact of the Ogallala formation with the underlying Pierre shale southeast of town.
The yield of approximately $10 \mathrm{gpm}$ is collected in a concrete reservoir and thence is pumped to the town.

Haxtun

Haxtun is supplied from 2 wells. Well B847-2lcc, at the power plant, is 233 feet deep. The static water leyel in this well is 150 feet below the land surface and the yield of the well is $565 \mathrm{gpm}$. Well B8-47-29aa is near the railroad and is 225 feet deep. The static water level is 142 feet below the land surface and the well yields $500 \mathrm{gpm}$. Both wells are equipped with electrically driven turbine pumps. Storage is provided at the power plant by an elevated tank. The water is pumped both directly into the mains and into the storage tank. No data on the consumption were obtainable.

Holyoke

Holyoke is supplied from 2 wells, well B743-17bc at the city park and well B7-44-7dd at the power plant. The depth of well B7-43 $-17 \mathrm{bc}$ is 265 feet, the static water level is 139 feet below land surface, and the yield is $625 \mathrm{gpm}$. Well B7-44-7dd is 223 feet deep, the static water level is 129 feet below the land surface, and the yield is $500 \mathrm{gpm}$. Both wells are equipped with electrically driven turbine pumps. Storage is provided by an 85,000-gallon standpipe in the city park. The water is pumped directly into the mains.

Otis

Otis is supplied from 1 well (B2-50-9dd), which is 233 feet deep and at the west end of town. The static water level in the well is 220 feet below the land surface and the yield is $300 \mathrm{gpm}$. The well is equipped with an electrically driven turbine pump. Storage is provided by a surface reservoir at the water 
works and an elevated tank at the city hall; the combined storage capacity is 105,000 gallons. The water is pumped directly into the mains and into the surface reservoir. The average daily consumption is 70,000 gallons.

Seibert

Seibert is supplied from 3 wells. Wells c9-49-3bci and -3bd are equipped with electrically driven cylinder pumps and well $\mathrm{C9}$ 49-3bc2 is equipped with an electrically driven turbine pump. The depth of the wells is about 150 feet, and the water level in the wells is about 140 feet below the land surface. Wells C9-49-3bcl, $-3 b c 2$, and $-3 b d$ are reported to yield about $300 \mathrm{gpm}, 600 \mathrm{gpm}$, and $125 \mathrm{gpm}$, respectively. Storage is provided by a 50,000-gallon standpipe northeast of town. The water is pumped directly into the mains and chlorine is used as a safeguard. The average daily consumption is about 30,000 gallons.

\section{Stratton}

Stratton is supplied from 3 wells. Wells c8-47-36cal and -36ca2 were drilled in 1922 and are 30 feet apart; well C9-49-1ba was drilled in 1949. All are equipped with electrically driven turbine pumps. The depth of well c9-47-lba was reported to be about 230 feet, the water level slightly more than 150 feet, and the yield about $500 \mathrm{gpm}$. Comparative data for the other wells were unobtainable. The water is stored in a 50,000gallon elevated tank. The older wells pump into the tank, and the newer well pumps directly into the mains. The average daily consumption is reported to be 26,000 gallons.

Vona

Vona is supplied from 2 wells (c9-49-2bbl and $-2 b b 2)$ that are equipped with windoperated cylinder pumps, and from 1 well (c9-48-2bb3) that is equipped with an electrically driven turbine pump, which is used when the wind is not blowing sufficiently for the pumps on the other wells to operate. Well c9-48-2bb3 is 80 feet deep, and the static water level in the well is 60 feet below the land surface. The yield is reported to be $300 \mathrm{gpm}$. Generally, the water is pumped directly into the mains; however, a tank is used for storage. The average daily consumption is reported to be 10,800 gallons.

Wray

Wray is supplied by 5 wells (Bl-43-6bc, $-6 b d 1,-6 b d 2,-6 b d 3$, and $-6 b d 4)$. The wells are about 75 feet deep, the static water level in the wells is about 60 feet below the land surface, and the yields range from 97 to $250 \mathrm{gpm}$. The wells are equipped with electrically driven turbine pumps. Storage is provided by a cemented dug pit south of town, which has a capacity of 300,000 gallons.

Yuma

Yuma is supplied from 2 wells in the northwestern part of town. Both wells are about 315 feet deep, and the static water level in the wells is about 180 feet below land surface. Well B2-48-22acl yields $300 \mathrm{gpm}$, and well B2-48-22ac2 yields $500 \mathrm{gpm}$. Both are equipped with electrically driven turbine pumps. Storage is provided by a surface reservoir northwest of town and a tank south of town; the combined storage capacity is 200,000 gallons. An average of 400,000 gallons of water is used daily.

\section{OBSERVATION WELIS}

Fluctuations of the water level in wells reflect the changes of the amount of ground water in storage. The principal sources of recharge to the ground-water reservoirs in this region are precipitation that reaches the water table by downward percolation and influent seepage from streams, especially in times of flood. Ground water is discharged from storage by effluent seepage into streams; by evaporation and transpiration, particularly 
where the water table is near the surface; by the flow of water from springs; by pumping from wells; and by subsurface flow out of the region.

Twenty-two wells in the region were selected for use in water-level observations. Most of these wells are in the areas of the greatest concentration of irrigation wells. Ten of the observation wells are abandoned stock or domestic wells, one is a stock well, and the remainder are irrigation wells. Monthly measurements of the water level have been made, but the measurements to date (1951) indicate no significant trend on a regional scale. Pertinent data for all observation wells are listed in table 2 . The location of the wells is shown on plate 1 .

\section{CONCLUSIONS}

The ground-water resources have not been fully developed in any part of the Kansas River basin in Colorado. Little intensive irrigation is practiced in the region; most irrigation water is used to supplement rainfall, and it is used only at a few critical times during the growing season. More pumping plants could be installed and operated in the present areas of irrigation concentration without excessive lowering of ground-water levels. However, as much information as possible on ground-water conditions should be collected while irrigation in the region is being developed, so as to provide a basis for orderly development.

In order to determine the perennial yield of ground water in the region, a detailed ground-water investigation would be needed. A water-table contour map.should be prepared for at least the major stream valleys; waterlevel measurements should be continued indefinitely; the geology should be mapped; maps showing the depth to water and the saturated thickness should be prepared; pumping tests should be made in order to determine the transmissibility and storage coefficients of the aquifers; and the effect of pumping on stream flow should be determined. A program of test drilling would be necessary in those areas where little or no information on subsurface conditions is available. A detailed appraisal of ground- water conditions in the relatively unproved areas would be particularly essential. For example, in many places in the area of dune sand north of Wray the soil is maturely developed, but the depth to water and the thickness of water-bearing materials are unknown.

\section{LITERATURE CITED}

Darton, N. H., and others, 1915, Guidebook of the western United States, Part C, the Santa Fe route: U. S. Geol. Survey Bull. $613,194 \mathrm{p}$.

Elias, M. K., 1931, The geology of the Wallace County, Kans.: Kansas Geol. Survey Bull. 18, $254 \mathrm{p}$.

Fenneman, N. M., 1931, Physiography of western United States: 534 p., New York, McGraw-Hill Book Co., Inc.

Hay, Robert, 1895, Water resources of a portion of the Great Plains: U. S. Geol. Survey 16th Ann. Report, pt. 2, p. 535588.

Hill, D. R., and Tompkin, J. M., Areal and engineering geology of the Wray area, Colorado and Nebraska: U. S. Geol. Survey Bull. 1001, (in press).

Johnson, W. D., 1901, The High Plains and their utilization: U. S. Geol. Survey 2lst Ann. Report, pt. 4, p. 601-741.

Judson, Sheldon, 1950,. Depressions of the northern portion of the southern High Plains of eastern New Mexico: Geol. Soc. America Bull., v. 61, no. 3, p. 253-274.

Smith, H. T. U., 1940, Geologic studies in southeastern Kansas: Kansas Geol. Survey Bull. $34,212 \mathrm{p}$.

RECORDS OF WELLS

Data recorded at the time the wells were visited are tabulated in the following table. 


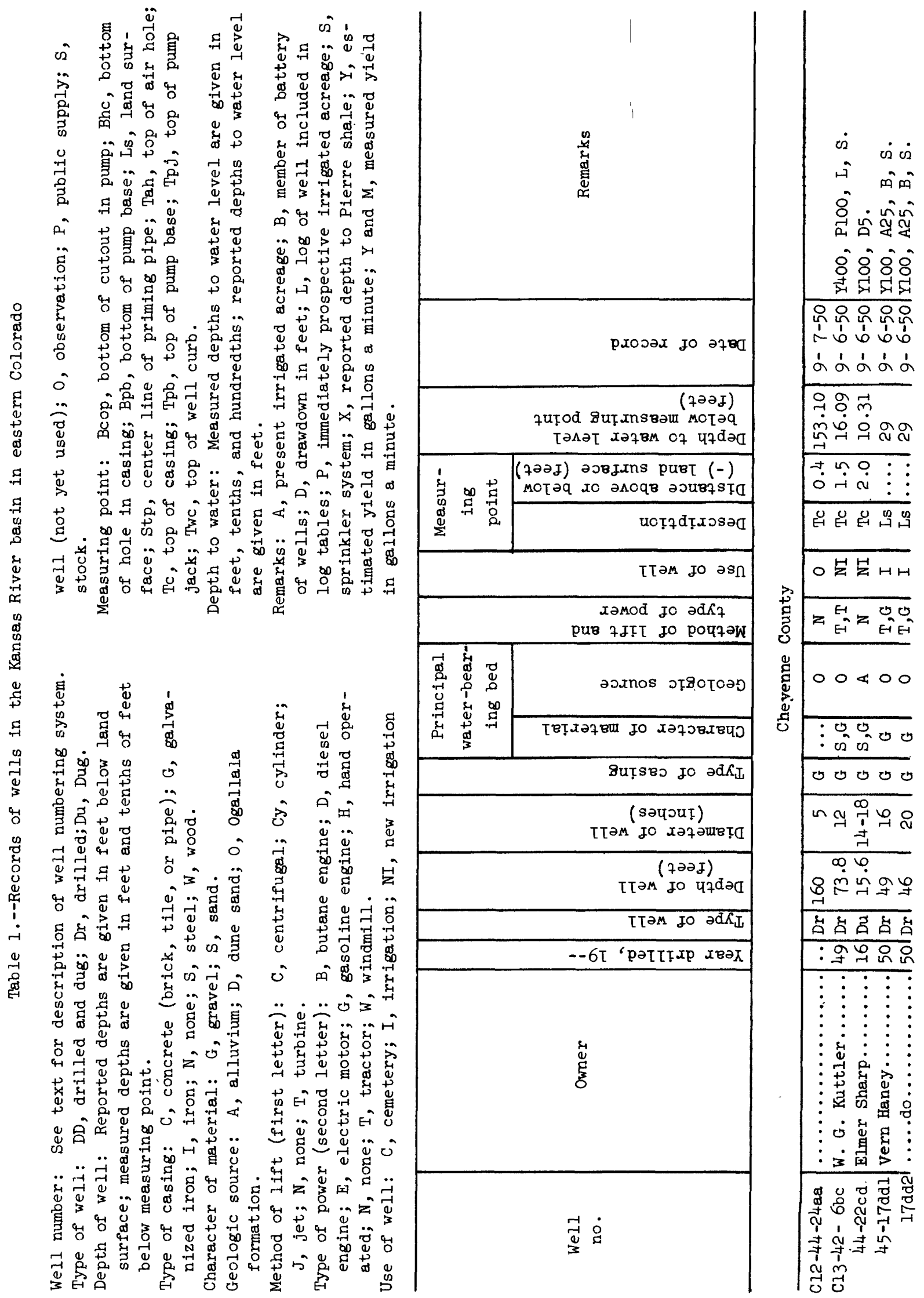




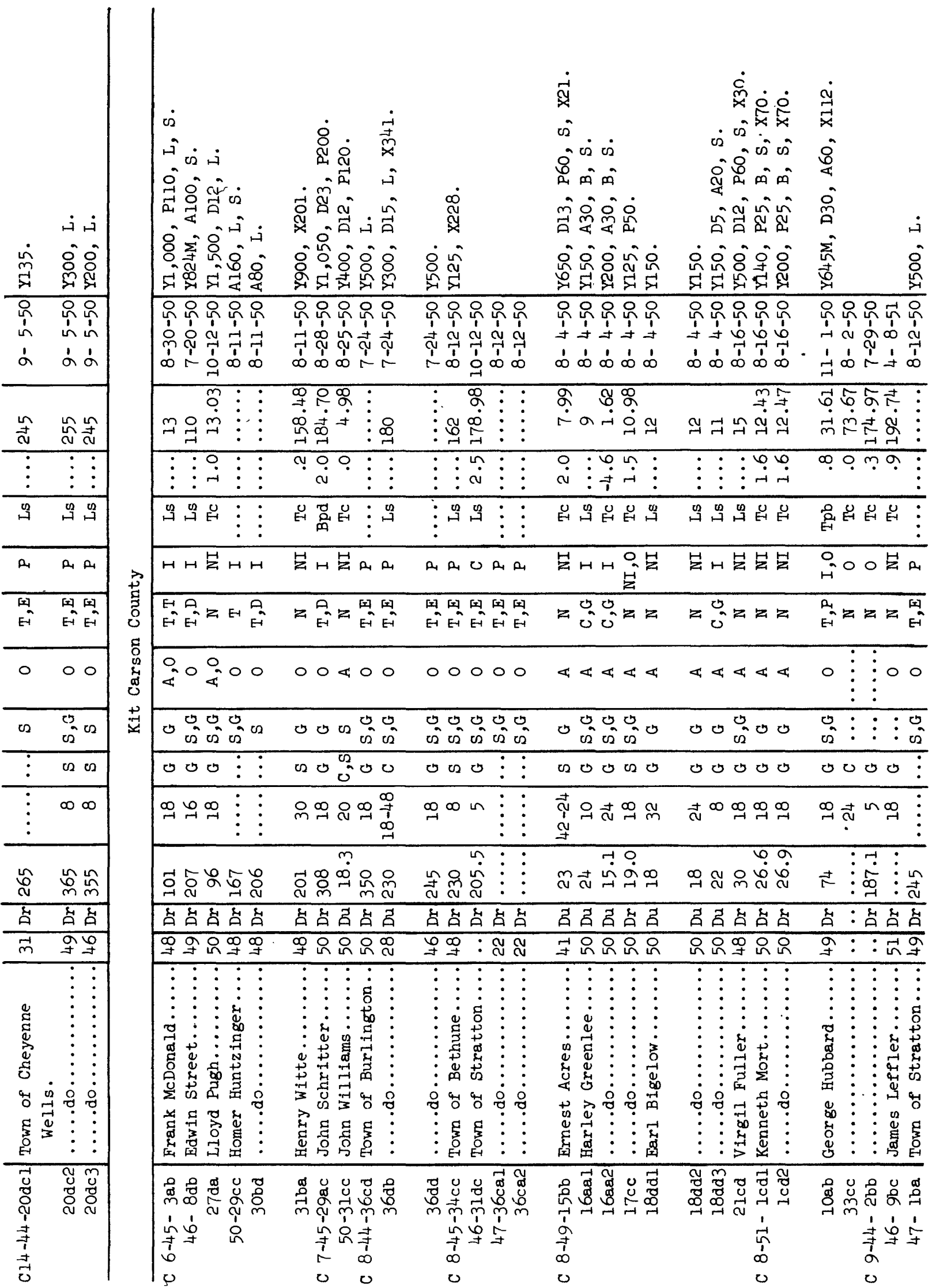




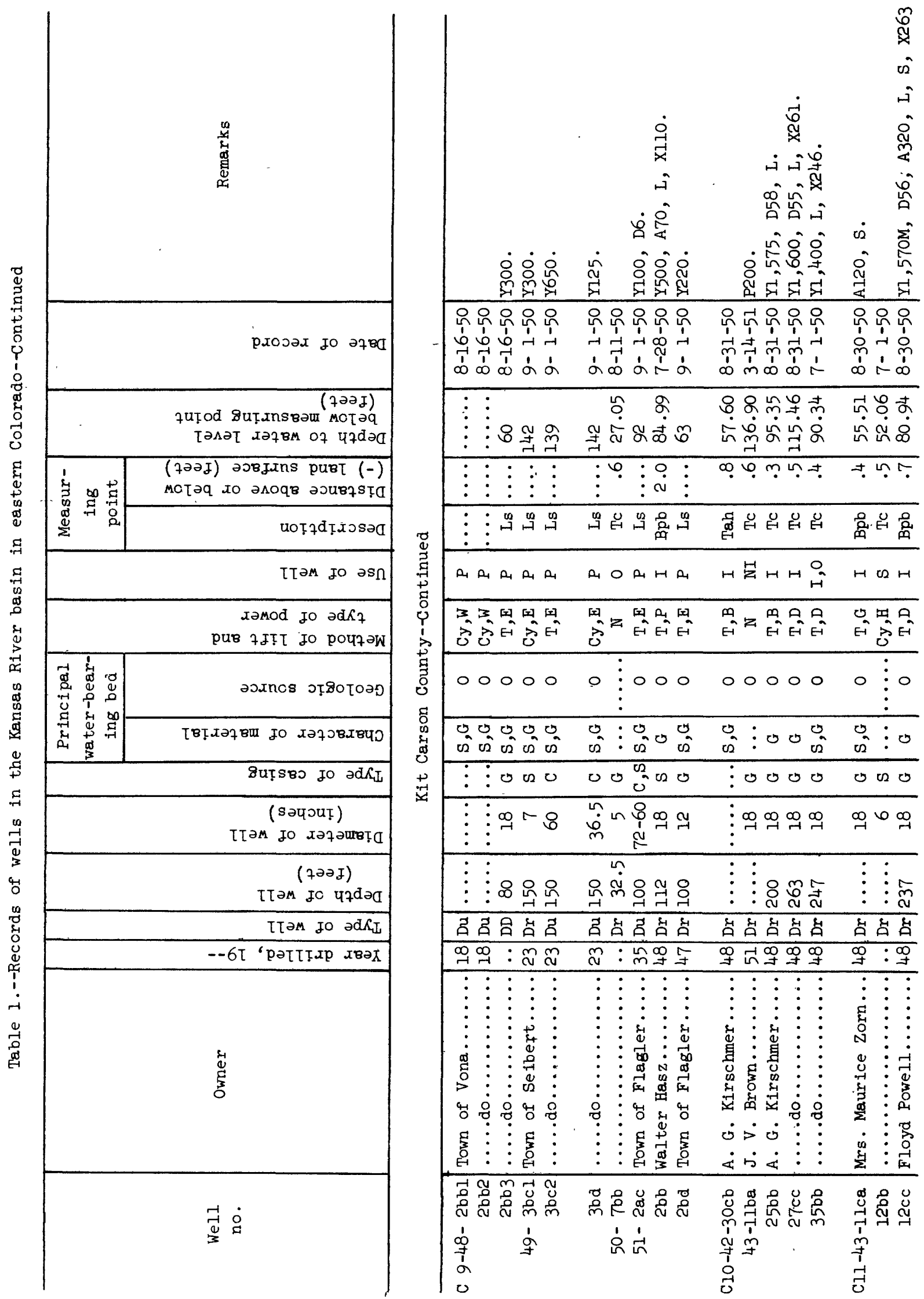




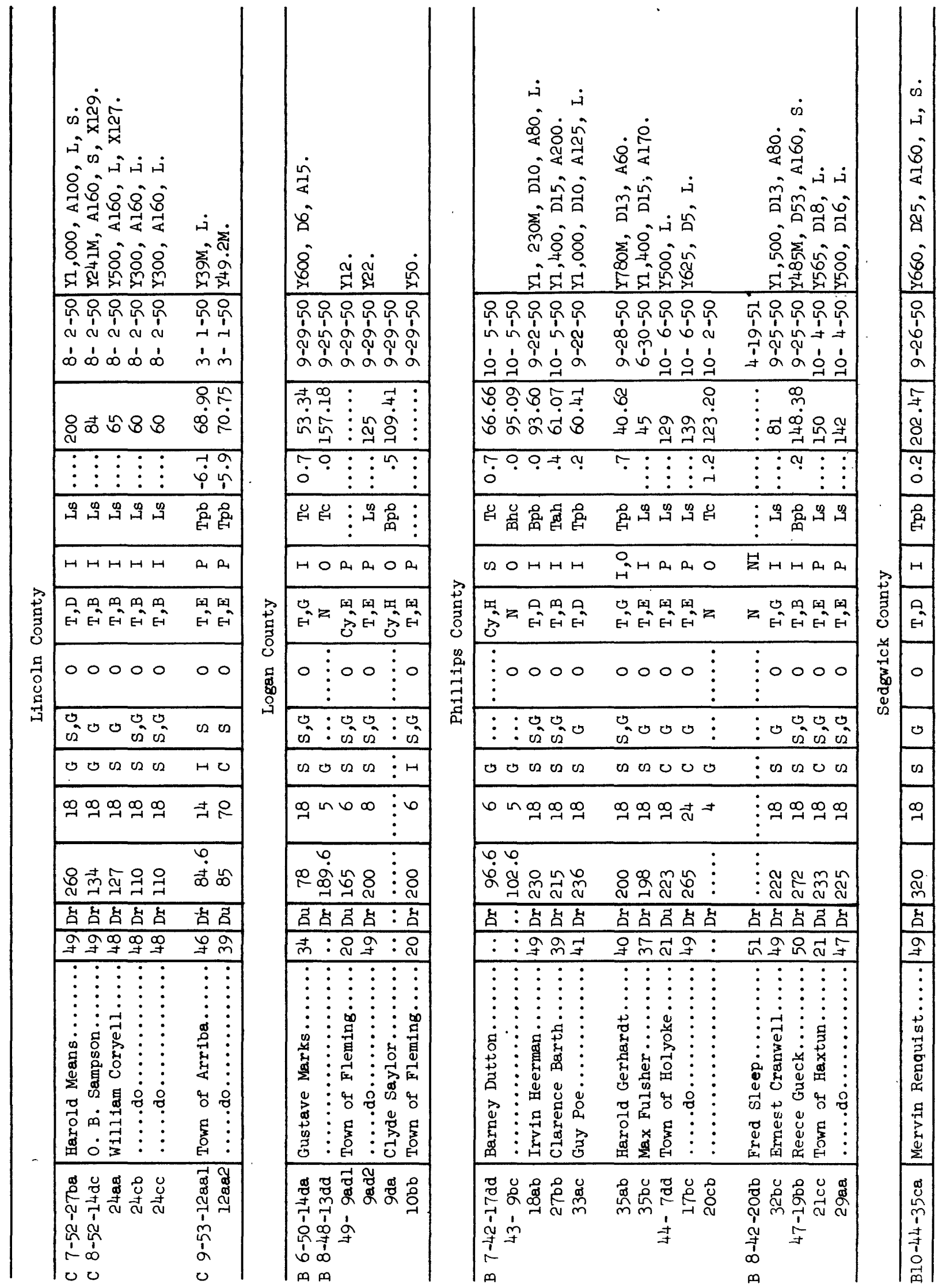




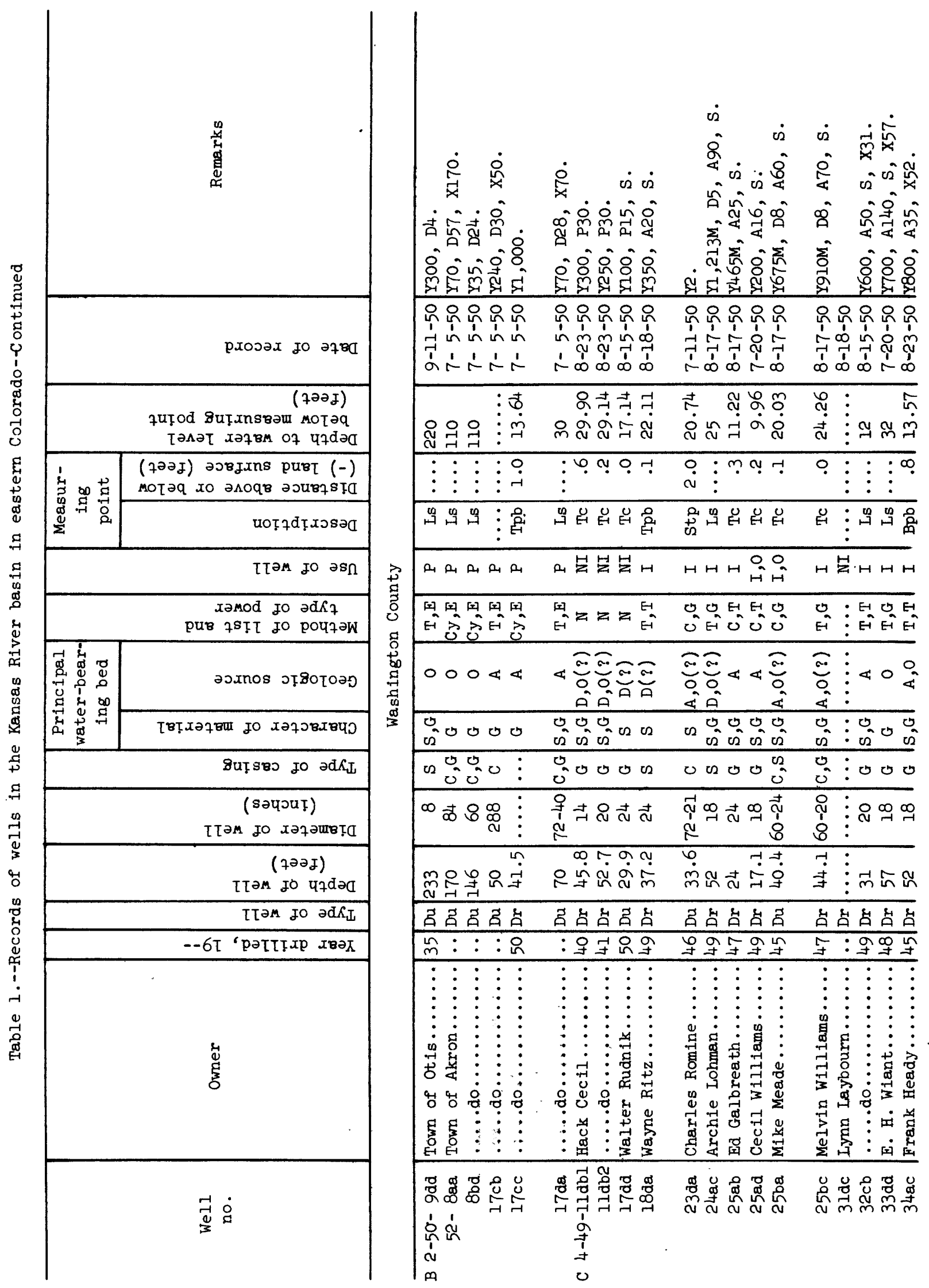




\begin{tabular}{|c|c|c|c|c|c|c|}
\hline $\begin{array}{l}\dot{0} \\
\dot{0} \\
\dot{0} \\
\dot{0}\end{array}$ & 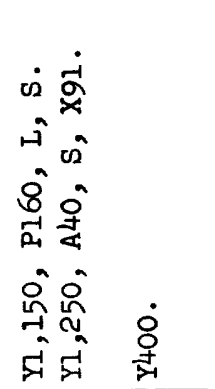 & 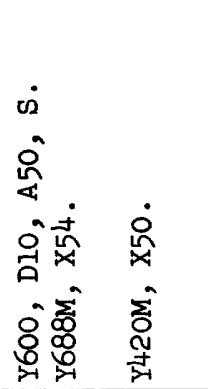 & 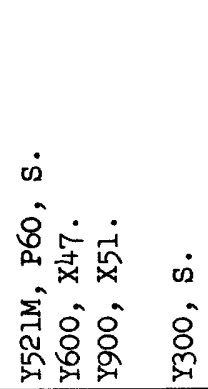 & 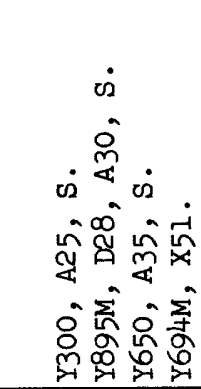 & 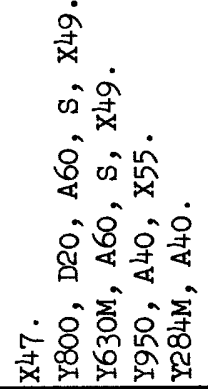 & 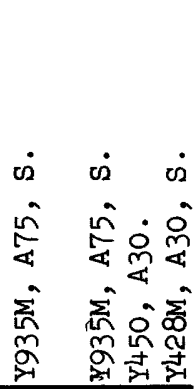 \\
\hline 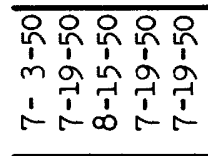 & 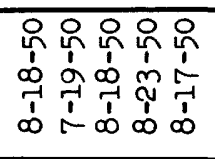 & 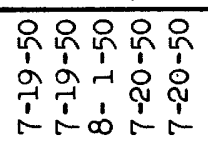 & 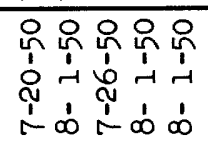 & 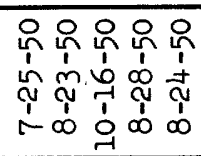 & 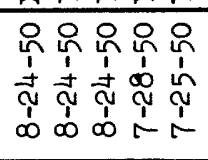 & 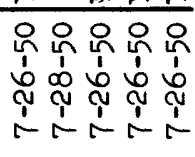 \\
\hline 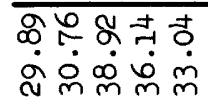 & 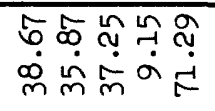 & 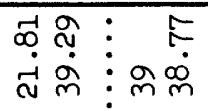 & 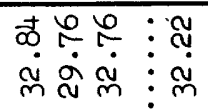 & 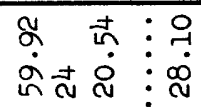 & 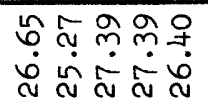 & 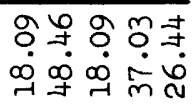 \\
\hline ㄷำ & $\infty \neq$ & 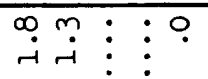 & न0 & 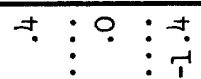 & & $96 \%$ \\
\hline 压 & 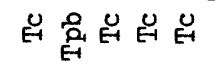 & 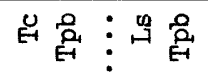 & 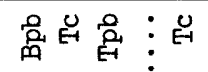 & 岙串总 & $\bigcup_{E-1}^{U}$ & 总品总总品 \\
\hline OHOHH & 总是吕。 & $\stackrel{\circ}{\circ} H$ 总HH & $H$ 芸H & OHH & & 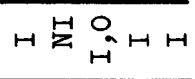 \\
\hline ZE & $Z_{E}^{E-1} z z z$ & 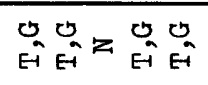 & $E_{-1}^{E-1}=E_{E-1}^{E-1}$ & 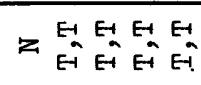 & $Z E-E$ & $\begin{array}{l}E-E E E \\
E=E A E\end{array}$ \\
\hline$\circ \vdots \circ \circ$ & ○ 0 ○只 & $\circ \circ \underset{\substack{0 \\
0}}{\pi} 0$ & ○ & 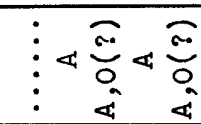 & ๔ã & 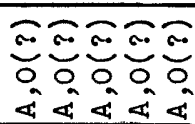 \\
\hline$\vdots_{02}^{0} \vdots 0_{0}^{0}$ & 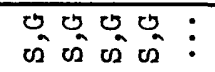 & 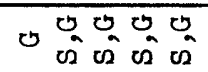 & 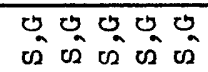 & 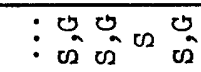 & 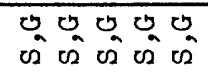 & 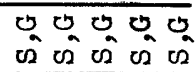 \\
\hline $0 \vdots 000$ & D 00000 & $00 \vdots 00$ & O 023 & DU & 0000000 & $0000_{0}^{0}$ \\
\hline 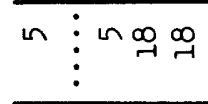 & 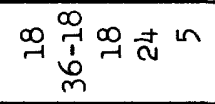 & तै ${ }^{\infty} \vdots \stackrel{\infty}{\infty} \underset{\sim}{\infty}$ & 尺 $\underset{\sim}{\infty} \stackrel{\sim}{\infty} \vdots \stackrel{\infty}{\infty}$ & 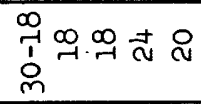 & 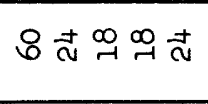 & 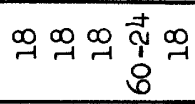 \\
\hline 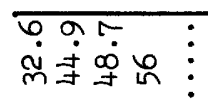 & ํㅏㅇ & 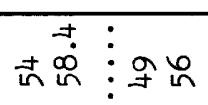 & 유유유 & $\vdots$ ₹ & 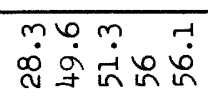 & 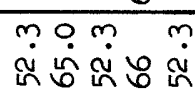 \\
\hline 苔䇗台合宫 & 䓃合台苔台 & 昌合合合尚 & 㕕䏍吕昌台 & : 台告台茼 & 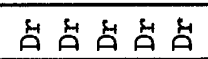 & 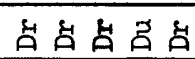 \\
\hline$:::$ : & 只示: 용: & 어엉 : : : & 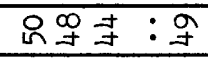 & 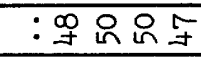 & 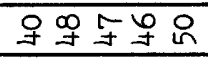 & 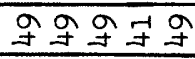 \\
\hline 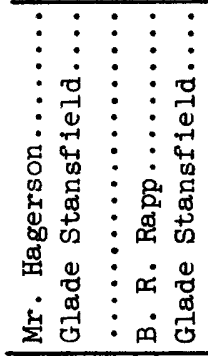 & 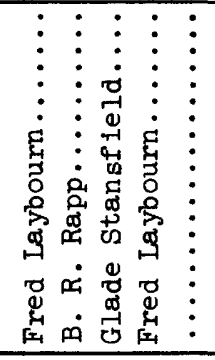 & 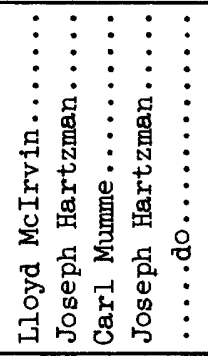 & 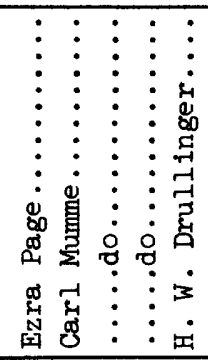 & 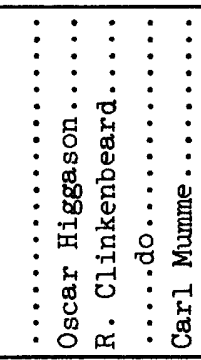 & 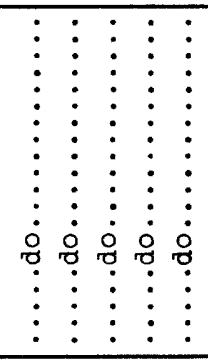 & 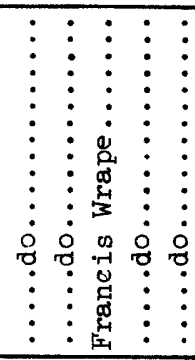 \\
\hline 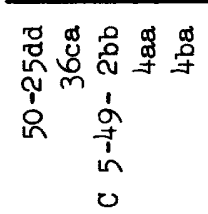 & 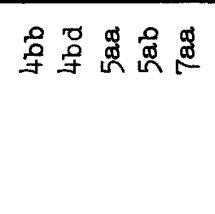 & 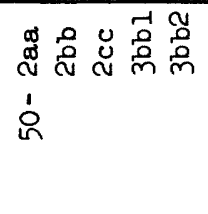 & 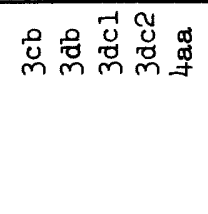 & 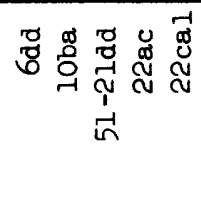 & 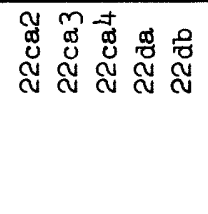 & 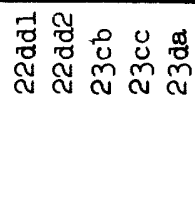 \\
\hline
\end{tabular}




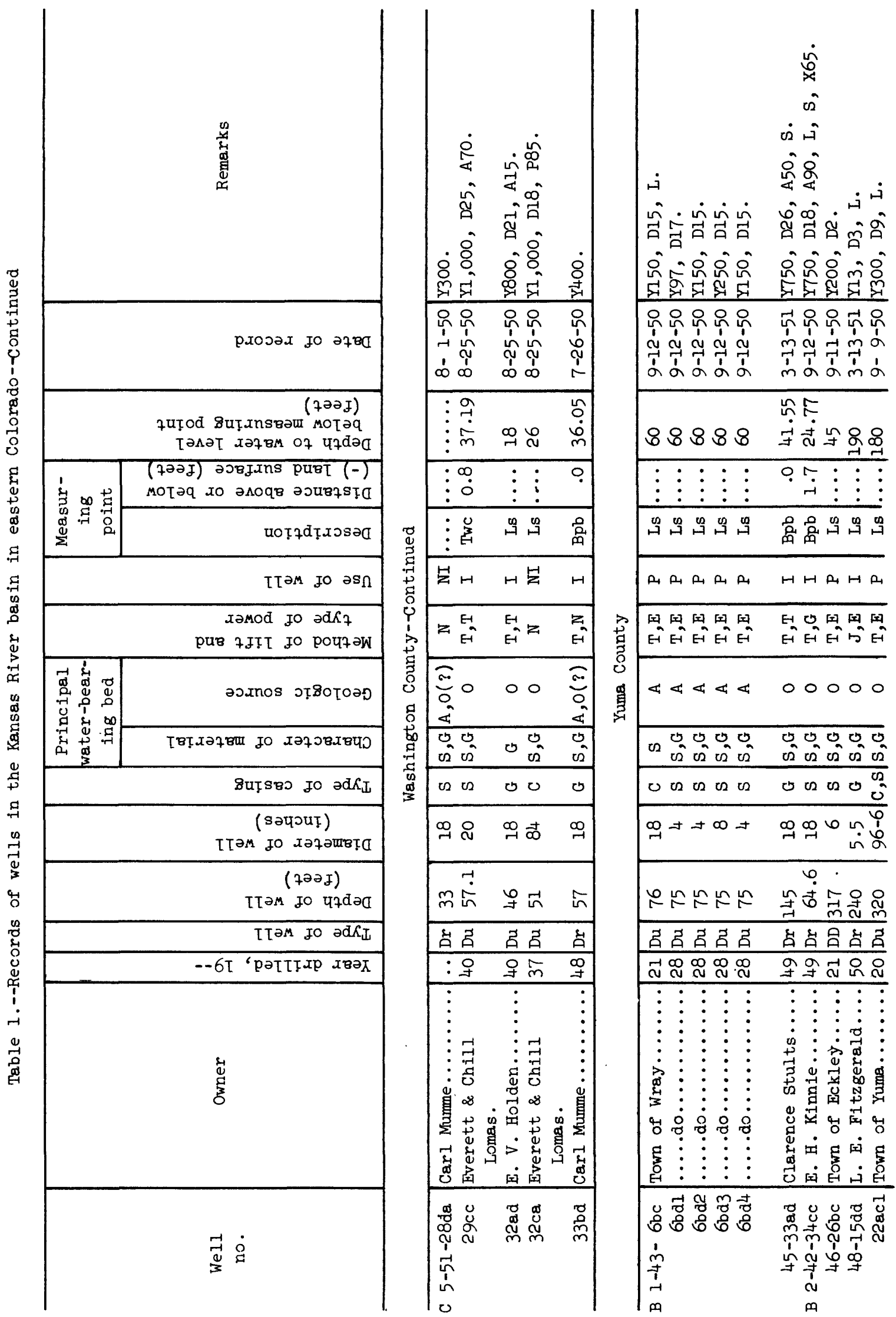




\begin{tabular}{|c|c|c|c|c|}
\hline 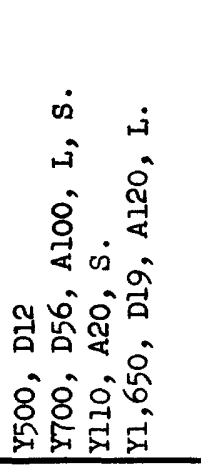 & 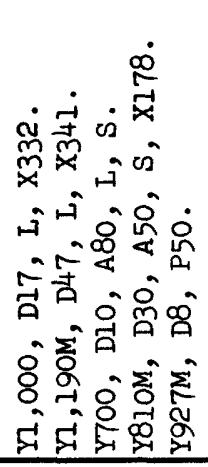 & 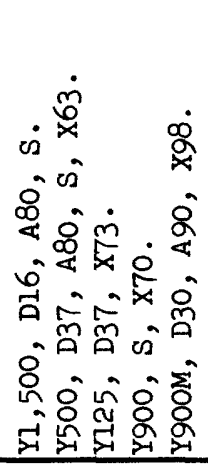 & 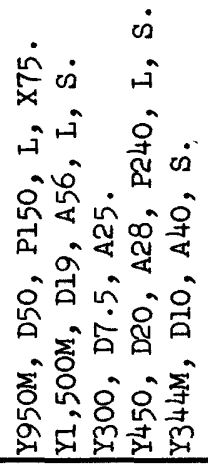 & 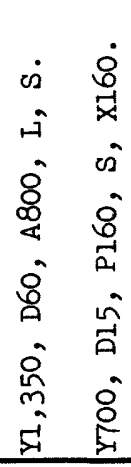 \\
\hline 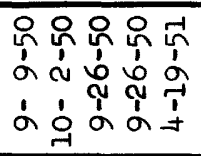 & 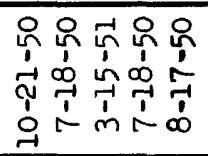 & 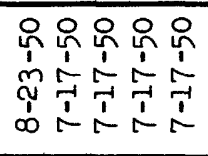 & 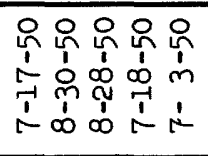 & 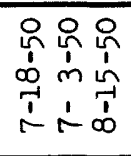 \\
\hline 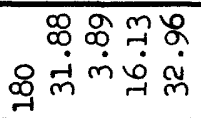 & 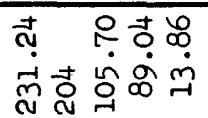 & 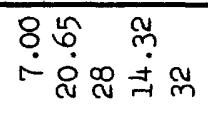 & 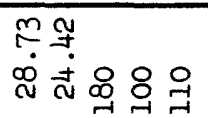 & 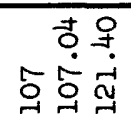 \\
\hline 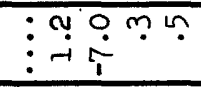 & 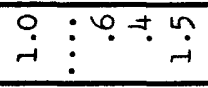 & 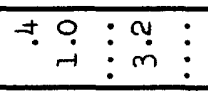 & ० $\stackrel{-1}{-1} \vdots \vdots \vdots$ & \\
\hline 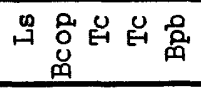 & 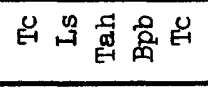 & 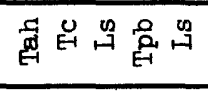 & 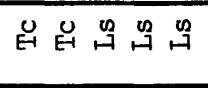 & ติ \\
\hline $\mathrm{A} \stackrel{\circ}{\mathrm{O}} \mathrm{H} H \mathrm{H}$ & 总HHH总 & $H H H H H$ & $\stackrel{O}{\mathrm{O}} H H H H$ & $H \cup 2 H$ \\
\hline 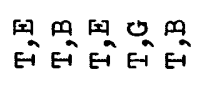 & $=\stackrel{9}{A}{ }_{E-1}^{M-1} E^{-1}$ & $\begin{array}{l}E A \text { A E- } A \\
\text { EI E- E- E- }\end{array}$ & $\begin{array}{l}O E O E N E \\
\text { EIE- E- E- }\end{array}$ & $A^{-}=E-F$ \\
\hline 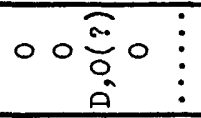 & $0000<$ & $<0000$ & 00000 & 0 \\
\hline 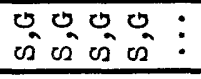 & $\begin{array}{lll}0 & 0 \\
0 & 0 & 0 \\
0\end{array}$ & 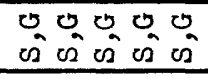 & $\begin{array}{llll}0 & 0 & 0 \\
01 & 0 & 0 \\
\end{array}$ & $\begin{array}{l:l}0 \\
0 \\
0\end{array}$ \\
\hline 0000 & 00000 & $00 H 0 \Omega$ & 00000 & 000 \\
\hline$\stackrel{्}{\infty} \underset{\sim}{\sim} \stackrel{\infty}{\vdots}$ & 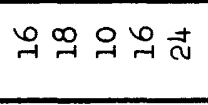 & $\stackrel{\infty}{\infty} \underset{\sim}{\infty} \infty \underset{m}{\infty} \underset{\sim}{\infty}$ & 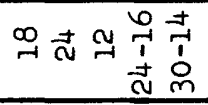 & $\stackrel{\infty}{\infty}$ in \\
\hline 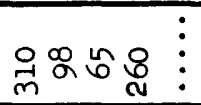 & 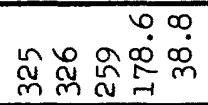 & $\begin{array}{l}+\infty \\
\dot{\alpha} \\
\stackrel{\alpha}{n} m\end{array}$ & 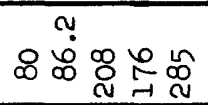 & 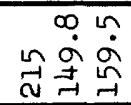 \\
\hline 台台：台： & 台台台台台 & 合台台苞䒤 & 䏍台台矣合 & 岗台䏍 \\
\hline 叧昌: & 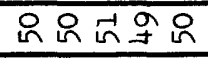 & 年等年星璐 & 员哭 & $9: 0$ \\
\hline 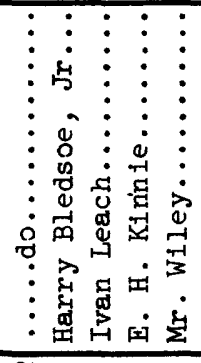 & 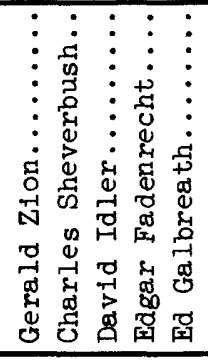 & 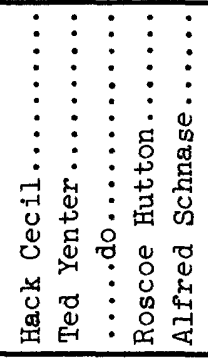 & 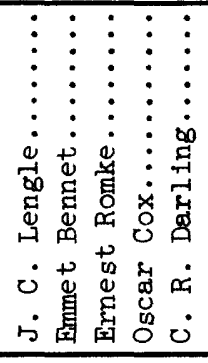 & 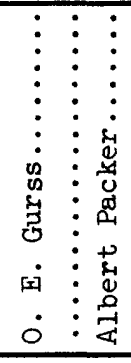 \\
\hline 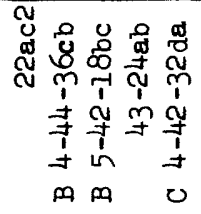 & 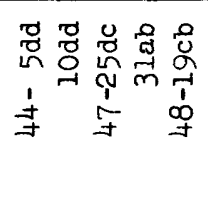 & 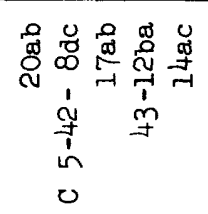 & 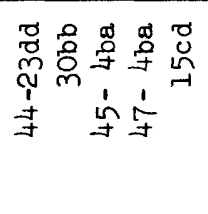 & 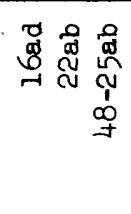 \\
\hline
\end{tabular}


LOGS OF TEST HOLES, SEISMOGRAPH SHOT HOLES, AND WELLS

The logs of 329 test holes, seismograph shot holes, and wells are presented in numerical order within counties on the following pages.

Well B8-47-29aa, a public-supply well for Haxtun, was drilled by L. L. Canfield in 1948; the log of the well cuttings was prepared by J.A. Tavelli of the U. S. Geological Survey. All of the other logs are drillers' logs. The logs of seismograph shot holes are of questionable value in subdividing lithologic units; their principal value is that they record where the top of the Pierre shale was reached.

The term "caliche" is substituted for "magnesia", "magnesia rock", "gyp rock", "lime", and "limestone", which are terms used by the drillers to describe certain calcareous beds. The term "sandstone" is used here as a substitute for the drillers' terms "sandstone", "sand rock", and "sandyrock"; the term "sandstone" is used variously for all sandy rocks from hard "mortar beds" to fairly well compacted or cemented sand. Formational names have been added to the drillers' logs.

Table.2.--Logs of test holes, selsmograph shot holes; and wells

Cheyenne County

\begin{tabular}{l|c|c}
\hline & $\begin{array}{c}\text { Thickness } \\
\text { (feet) }\end{array}$ & $\begin{array}{c}\text { Depth } \\
\text { (feet) }\end{array}$ \\
\hline
\end{tabular}

\section{C12-42-14dd}

\begin{tabular}{|c|c|c|}
\hline Soil, sandy........... & 5 & 5 \\
\hline Ogallala formation: & & \\
\hline $\begin{array}{l}\text { Sand, containing clay } \\
\text { breaks .............. }\end{array}$ & 60 & 65 \\
\hline 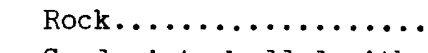 & 3 & 68 \\
\hline 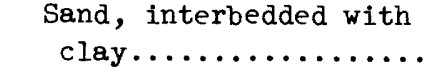 & 78 & 146 \\
\hline Sand (water).......... & 4 & 150 \\
\hline Pierre shale: & & \\
\hline Shale, blue.......... & $\ldots$ & 150 \\
\hline
\end{tabular}

Table 2.--Logs of test holes, selsmograph shot holes, and wells--Continued

Cheyenne County--Continued

\begin{tabular}{l|c|c}
\hline & $\begin{array}{c}\text { Thickness } \\
\text { (feet) }\end{array}$ & $\begin{array}{c}\text { Depth } \\
\text { (feet) }\end{array}$ \\
\hline
\end{tabular}

C12-42-22. (South half of section)

\begin{tabular}{|c|c|c|}
\hline 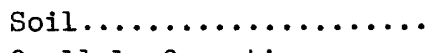 & 15 & 15 \\
\hline Ogallala formation: & & \\
\hline Rock............... & 65 & 80 \\
\hline Sand............... & 6 & 86 \\
\hline 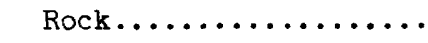 & 44 & 130 \\
\hline 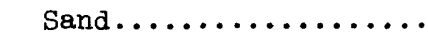 & 8 & 138 \\
\hline 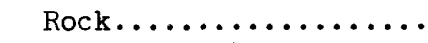 & 27 & 165 \\
\hline Sand (water).......... & 10 & 175 \\
\hline Pierre shale: & & \\
\hline 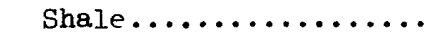 & $\cdots$ & 175 \\
\hline
\end{tabular}

C12-44-6. (West half of section)

\begin{tabular}{c|r|r}
\hline Ogallala formation: & & \\
Clay with some streaks & & \\
of sand........... & 140 & 140 \\
Soapstone, fairly soft. & 10 & 150 \\
Gravel (water)....... & 8 & 158 \\
Pierre shale: & & \\
Shale.............. & $\ldots$ & 158 \\
\hline
\end{tabular}

C13-42-6bc. Drilled by John Snyder, 1949

\begin{tabular}{|c|c|c|}
\hline 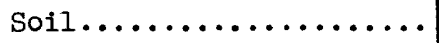 & 8 & 8 \\
\hline $\begin{array}{l}\text { Soil, sandy } \ldots \ldots \ldots \ldots \ldots \ldots \\
\text { Ogallala formation: }\end{array}$ & 7 & 15 \\
\hline Clay and sand......... & 15 & 30 \\
\hline Sand, good (water)..... & 18 & 48 \\
\hline Clay and gravel....... & 22 & 70 \\
\hline Gravel (water)........ & 10 & 80 \\
\hline Sandstone............ & 2 & 82 \\
\hline
\end{tabular}

C13-43-24a

\begin{tabular}{|c|c|c|}
\hline Soil.................. & 4 & 4 \\
\hline $\begin{array}{l}\text { Ogallala formation: } \\
\text { Sand, alternating with }\end{array}$ & & \\
\hline clay.............. & 196 & 200 \\
\hline $\begin{array}{l}\text { Rock............... } \\
\text { Sand, alternating with }\end{array}$ & 2 & 202 \\
\hline 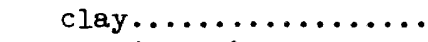 & 66 & 268 \\
\hline Sand (water)......... & 2 & 270 \\
\hline Pierre shale: & & \\
\hline Shale, blue........... & $\cdots$ & 270 \\
\hline
\end{tabular}


Table 2.--Logs of test holes, seismograph shot holes, and wells--Continued

Cheyenne County--Continued

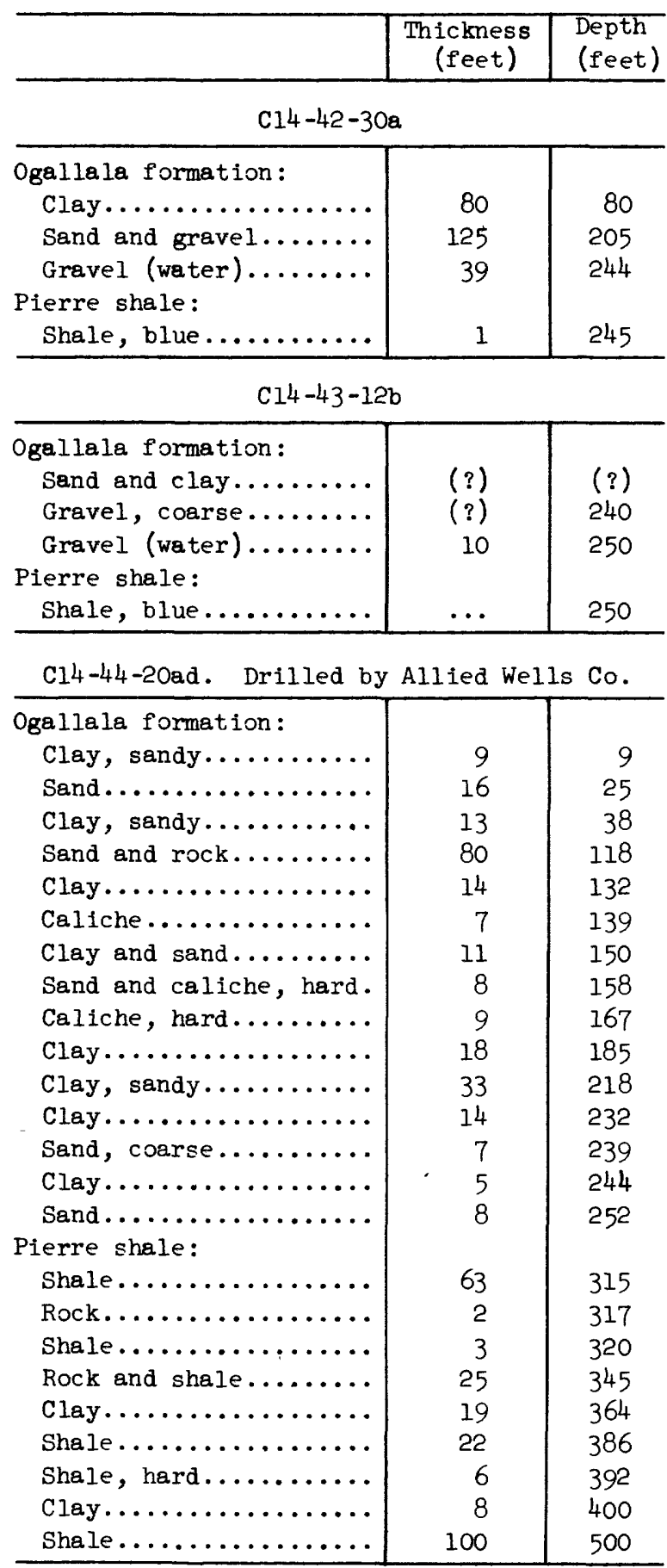

Table 2.--Logs of test holes, seismograph shot holes, and wells--Continued

Cheyenne County--Continued

\begin{tabular}{l|c|c}
\hline & $\begin{array}{c}\text { Thickness } \\
\text { (feet) }\end{array}$ & $\begin{array}{c}\text { Depth } \\
\text { (feet) }\end{array}$ \\
\hline
\end{tabular}

C14-44-20dc2. Drilled by Jack Doty, 1949

\begin{tabular}{|c|c|c|}
\hline Soil................. & 15 & 15 \\
\hline Clay, sandy............ & 10 & 25 \\
\hline 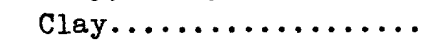 & 10 & 35 \\
\hline Clay, firm, sticky.... & 10 & 45 \\
\hline 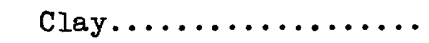 & 5 & 50 \\
\hline Clay, sandy.......... & 5 & 55 \\
\hline 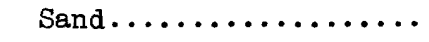 & 15 & 70 \\
\hline Clay, sandy........... & 15 & 85 \\
\hline 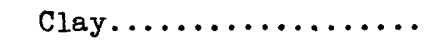 & 30 & 115 \\
\hline Clay, sandy........... & 5 & 120 \\
\hline 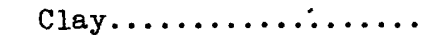 & 15 & 135 \\
\hline Clay, sandy.......... & 20 & 155 \\
\hline 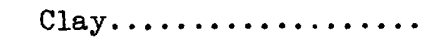 & 5 & 160 \\
\hline 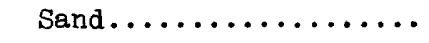 & 15 & 175 \\
\hline Sand and clay......... & 20 & 195 \\
\hline 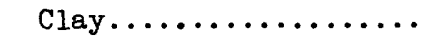 & 15 & 210 \\
\hline Sand and clay......... & 5 & 215 \\
\hline Gravel, coarse........ & 24 & 239 \\
\hline 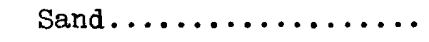 & 6 & 245 \\
\hline 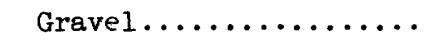 & 30 & 275 \\
\hline 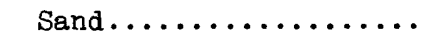 & 85 & 360 \\
\hline
\end{tabular}

C14-44-20dc3. Drilled by Otis Shuck, 1946

\begin{tabular}{|c|c|c|}
\hline $\begin{array}{l}\text { Soil, sandy ............ } \\
\text { Ogallala: formation: }\end{array}$ & 10 & 10 \\
\hline Clay, sandy.......... & 10 & 20 \\
\hline Clay and caliche...... & 40 & 60 \\
\hline Sand and clay......... & 40 & 100 \\
\hline Sand, fine........... & 20 & 120 \\
\hline 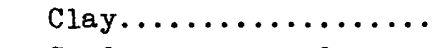 & 10 & 130 \\
\hline $\begin{array}{l}\text { Sand, coarse, and } \\
\text { gravel } \ldots \ldots \ldots \ldots \ldots\end{array}$ & 10 & 140 \\
\hline Sand and gravel....... & 10 & 150 \\
\hline Sand, fine........... & 30 & 180 \\
\hline Sand, medium.......... & 10 & 190 \\
\hline Rock, hard, and gravel. & 10 & 200 \\
\hline 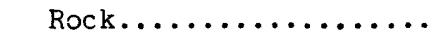 & 10 & 210 \\
\hline Clay and rock.......... & 10 & 220 \\
\hline 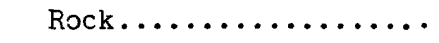 & 10 & 230 \\
\hline $\begin{array}{l}\text { Sand, compact; gravel, } \\
\text { and rock............. }\end{array}$ & 10 & 240 \\
\hline
\end{tabular}


Table 2.--Logs of test holes, seismograph shot holes, and wells--Continued

Cheyenne County--Continued

\begin{tabular}{l|c|c}
\hline & $\begin{array}{c}\text { Thickness } \\
\text { (feet) }\end{array}$ & $\begin{array}{c}\text { Depth } \\
\text { (feet) }\end{array}$ \\
\hline
\end{tabular}

\section{C14-44-20dc3--Continued}

\begin{tabular}{|c|c|c|}
\hline \multicolumn{3}{|l|}{ Ogallala formation--Con. } \\
\hline Clay................ & 5 & 245 \\
\hline Clay, soft.......... & 10 & 255 \\
\hline Sand (water)......... & 15 & 270 \\
\hline 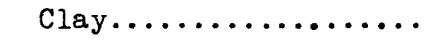 & 10 & 280 \\
\hline Sand (water) ......... & 10 & 290 \\
\hline $\begin{array}{l}\text { Sand, fine............ } \\
\text { Sand, fine, containing }\end{array}$ & 10 & 300 \\
\hline some clay........... & 30 & 330 \\
\hline Clay, compact......... & 5 & 335 \\
\hline Sand, good (water)..... & 10 & 345 \\
\hline Pierre shale: & - & \\
\hline Shale, yellow and & & \\
\hline green $\ldots \ldots \ldots \ldots \ldots$ & 10 & 355 \\
\hline
\end{tabular}

C15-42-25c

\begin{tabular}{|c|c|c|}
\hline \multicolumn{3}{|l|}{$\begin{array}{l}\text { Ogallala formation: } \\
\text { Clay, containing a }\end{array}$} \\
\hline $\begin{array}{l}\text { little sand............... } \\
\text { Quicksand, very wet... }\end{array}$ & $\begin{array}{r}175 \\
1\end{array}$ & $\begin{array}{l}175 \\
176\end{array}$ \\
\hline Clay............... & 29 & 205 \\
\hline Sand, fine (water)..... & 5 & 210 \\
\hline Pierre shale: & & \\
\hline Shale, blue.......... & $\cdots$ & 210 \\
\hline
\end{tabular}

\section{C15-44-5}

\begin{tabular}{|c|c|c|}
\hline $\begin{array}{l}\text { Soil .................. } \\
\text { Ogallala formation: }\end{array}$ & 4 & 4 \\
\hline Caliche.............. & 2 & 6 \\
\hline Clay, sandy.......... & 209 & 215 \\
\hline Quicksand............ & 45 & 260 \\
\hline Rock............. & 5 & 265 \\
\hline Grave1, coarse (water). & 5 & 270 \\
\hline Pierre shale: & & \\
\hline Shale, blue.......... & ... & 270 \\
\hline
\end{tabular}

\section{Kit Carson County}

\begin{tabular}{c|c|c}
\hline & $\begin{array}{c}\text { Thickness } \\
\text { (feet) }\end{array}$ & $\begin{array}{c}\text { Depth } \\
\text { (feet) }\end{array}$ \\
\hline
\end{tabular}

C6-45-3ab. Drilled. by K. G. Wilcox, 1948

\begin{tabular}{|c|c|c|}
\hline Soil, clayey.......... & 17 & 17 \\
\hline $\begin{array}{l}\text { Alluvium(?): } \\
\text { Clay, containing strips }\end{array}$ & 3 & 0 \\
\hline Gravel, coarse......... & 12 & 32 \\
\hline
\end{tabular}

Table 2.--Logs of test holes, seismograph shot holes, and wells--Continued

Kit Carson County--Continued

\begin{tabular}{l|c|c}
\hline & $\begin{array}{c}\text { Thickness } \\
\text { (feet) }\end{array}$ & $\begin{array}{c}\text { Depth } \\
\text { (feet) }\end{array}$ \\
\hline
\end{tabular}

c6-45-3ab--Continued

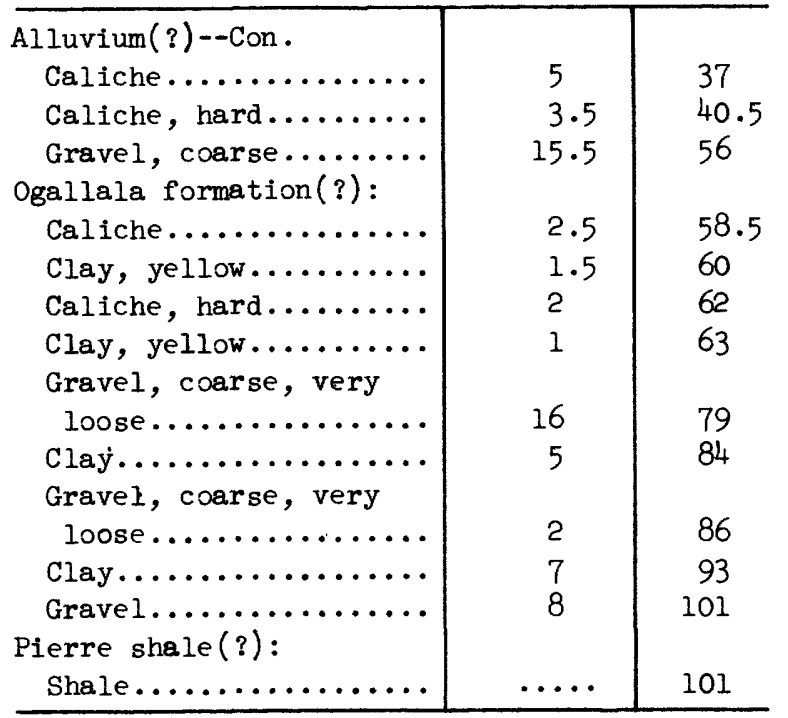

C6-46-27da. Drilled by K. G. Wilcox, 1950

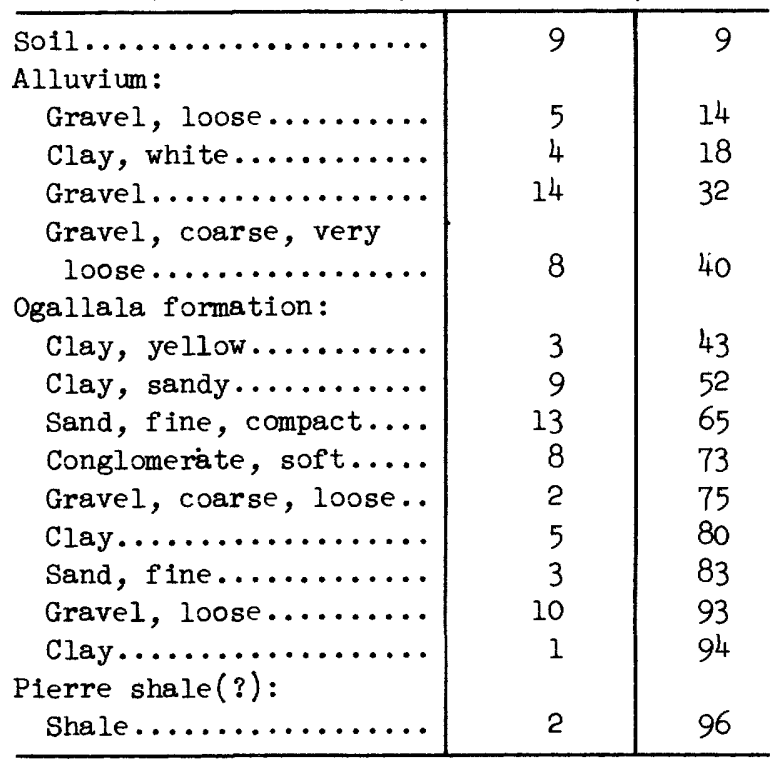

\section{$\mathrm{c} 6-50-6 \mathrm{bb}$}

\begin{tabular}{|c|c|c|}
\hline Ogallala formation: & & \\
\hline 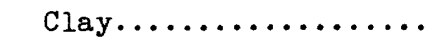 & 40 & 40 \\
\hline Gravel and sand....... & 50 & 90 \\
\hline (No sample) ........... & 10 & 100 \\
\hline Sand............... & 40 & 140 \\
\hline Clay, yellow.......... & 25 & 165 \\
\hline
\end{tabular}


Table 2.--Logs of test holes, seismograph shot holes, and wells--Continued

Kit Carson County--Continued

\begin{tabular}{l|c|c}
\hline & $\begin{array}{c}\text { Thickness } \\
\text { (feet) }\end{array}$ & $\begin{array}{c}\text { Depth } \\
\text { (feet) }\end{array}$ \\
\hline
\end{tabular}

C6-50-6bb--Continued

\begin{tabular}{|c|c|c|}
\hline $\begin{array}{l}\text { Pierre shale: } \\
\text { Shale, blue ........... }\end{array}$ & 5 & 170 \\
\hline c6-50-29cc. & Ben Hasz, & 1948 \\
\hline Soil............. & 8 & 8 \\
\hline Ogallala formation: & & \\
\hline $\begin{array}{l}\text { Sand, containing strips } \\
\text { of caliche............. }\end{array}$ & 123 & 131 \\
\hline Caliche, containing & & \\
\hline strips of sand........ & 10 & 141 \\
\hline Sand, loose........... & 5 & 146 \\
\hline Caliche and sand....... & 1 & 147 \\
\hline Sand, loose........... & 2 & 149 \\
\hline Clay, sandy...$\ldots \ldots \ldots$ & 3 & 152 \\
\hline Gravel, loose....... & 8 & 160 \\
\hline Clay, yellow........... & 7 & 167 \\
\hline Pierre shale(?): & & \\
\hline Shale $\ldots \ldots \ldots \ldots \ldots$ & ... & 167 \\
\hline
\end{tabular}

C6-50-30bd. Drilled by Ben Hasz, 1948

\begin{tabular}{|c|c|c|}
\hline 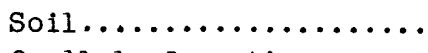 & 4 & 4 \\
\hline Ogallala formation: & & \\
\hline $\begin{array}{l}\text { Sand } . . . \ldots \ldots \ldots \ldots \\
\text { Clay, sandy, and sand- }\end{array}$ & 21 & 25 \\
\hline $\begin{array}{l}\text { stone } . . . \ldots \ldots \ldots \ldots \ldots \\
\text { Sand, with some cali- }\end{array}$ & 22 & 47 \\
\hline $\begin{array}{l}\text { che......................... } \\
\text { Sand, caliche, and }\end{array}$ & 17 & 64 \\
\hline 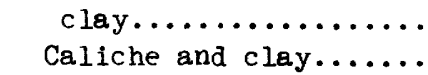 & $\begin{array}{l}23 \\
18\end{array}$ & $\begin{array}{r}87 \\
105\end{array}$ \\
\hline 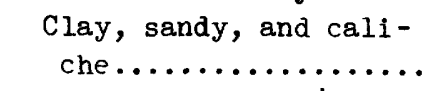 & 7 & 112 \\
\hline $\begin{array}{l}\text { Sand, with some clay... } \\
\text { Clay, sandy, and cali- } \\
\text { che; contains some } \\
\text { sandstone............ }\end{array}$ & 15 & 127 \\
\hline 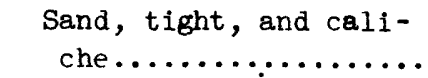 & 5 & 157 \\
\hline $\begin{array}{l}\text { Clay ........................ } \\
\text { Sand, fairly loose, } \\
\text { containing strips of }\end{array}$ & 10 & 167 \\
\hline 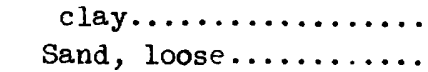 & $\begin{array}{l}21 \\
10\end{array}$ & $\begin{array}{l}188 \\
198\end{array}$ \\
\hline Clay, yellow............ & 8 & 206 \\
\hline Pierre shale(?): & & \\
\hline Shale......... & $\cdots$ & 206 \\
\hline
\end{tabular}

Table 2,--Logs of test holes, seismograph shot holes, and wells--Continued

Kit Carson County--Continued

\begin{tabular}{l|c|c}
\hline & $\begin{array}{c}\text { Thickness } \\
\text { (feet) }\end{array}$ & $\begin{array}{c}\text { Depth } \\
\text { (feet) }\end{array}$ \\
\hline
\end{tabular}

$$
\text { c6 }-51-6 b b
$$

\begin{tabular}{|c|c|c|}
\hline \multirow{2}{*}{\multicolumn{3}{|c|}{ Ogallala formation: }} \\
\hline & & \\
\hline Clay...$\ldots \ldots \ldots \ldots$ & 40 & 40 \\
\hline Gravel and sand........ & 50 & 90 \\
\hline Caliche............. & 10 & 100 \\
\hline Sand $\ldots . . . . . . . . .$. & 40 & 140 \\
\hline Clay, yellow........... & 15 & 155 \\
\hline \multicolumn{3}{|l|}{ Pierre shale: } \\
\hline Shale, blue........... & 5 & 160 \\
\hline \multicolumn{3}{|c|}{$c 7-42-15 d d$} \\
\hline silt................... & 10 & 10 \\
\hline \multirow{2}{*}{\multicolumn{3}{|c|}{$\begin{array}{l}\text { Ogallala formation: } \\
\text { Clay, sandy........... }\end{array}$}} \\
\hline & 50 & 60 \\
\hline Gravel.............. & 60 & 120 \\
\hline Sand, containing small & & \\
\hline & 80 & 200 \\
\hline Sand, with streaks of & & \\
\hline clay......... & 110 & 310 \\
\hline \multicolumn{3}{|l|}{ Pierre shale: } \\
\hline Shale, blue........... & 10 & 320 \\
\hline
\end{tabular}

\begin{tabular}{|c|c|c|}
\hline \multicolumn{3}{|c|}{$c 7-43-16 d d$} \\
\hline silt................... & 10 & 10 \\
\hline Ogallala formation: & & \\
\hline Clay, sandy............ & 50 & 60 \\
\hline Gravel............... & 40 & 100 \\
\hline $\begin{array}{l}\text { Gravel, with small } \\
\text { breaks of caliche..... }\end{array}$ & 180 & 280 \\
\hline Sand and clay.......... & 22 & 302 \\
\hline Pierre shale: & & \\
\hline Shale, blue........... & 8 & 310 \\
\hline
\end{tabular}

\section{C7 $-44-16 \mathrm{~d}$}

\begin{tabular}{l|r|r}
\hline Ogallala formation: & & \\
Sand and streaks of & & \\
gravel and caliche.... & 195 & 195 \\
Clay, yellow......... & 10 & 205 \\
Pierre shale: & & \\
Shale, blue........... & 15 & 220 \\
\hline
\end{tabular}

C7-44-31cc. Altitude, 4,157 feet

\begin{tabular}{|c|c|c|}
\hline Ogallala formation: & & \\
\hline Clay.$\ldots \ldots \ldots \ldots \ldots$ & 20 & 20 \\
\hline Grave1............. & 30 & 50 \\
\hline Clay and caliche....... & 140 & 190 \\
\hline Grave1............. & 75 & 265 \\
\hline
\end{tabular}


Table 2.--Logs of test holes, seismograph shot holes, and wells--Continued

Kit Carson County--Continued

\begin{tabular}{l|c|c}
\hline & $\begin{array}{c}\text { Thickness } \\
\text { (feet) }\end{array}$ & $\begin{array}{c}\text { Depth } \\
\text { (feet) }\end{array}$ \\
\hline
\end{tabular}

C7-44-31cc--Continued

\begin{tabular}{l|c|c}
\hline $\begin{array}{c}\text { Pierre shale: } \\
\text { Ch-45-2laa }\end{array}$ & 5 & 270 \\
\hline \multicolumn{2}{c|}{ Shale, blue........ } & 5 \\
\hline $\begin{array}{l}\text { Ogallala formation: } \\
\text { Clay and caliche...... } \\
\text { Clay, caliche, and } \\
\text { breaks of marl...... }\end{array}$ & 140 & 140 \\
$\begin{array}{l}\text { Pierre shale: } \\
\text { Shale, blue.......... }\end{array}$ & 15 & 315 \\
\hline
\end{tabular}

C7-45-29ac. Drilled by K. G. Wilcox, 1950

\begin{tabular}{|c|c|c|}
\hline $\begin{array}{l}\text { Soil.................. } \\
\text { Ogallala formation: }\end{array}$ & 52 & 52 \\
\hline $\begin{array}{l}\text { Caliche, hard, blocky.. } \\
\text { Caliche, compact, and }\end{array}$ & 2 & 54 \\
\hline $\begin{array}{l}\text { Gravel, fine, compact.. } \\
\text { Gravel, coarse, very } \\
\text { compact.............. }\end{array}$ & 2 & 94 \\
\hline 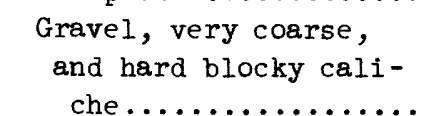 & 3 & 99 \\
\hline $\begin{array}{l}\text { Caliche and sandy com- } \\
\text { pact clay............ }\end{array}$ & 1 & 100 \\
\hline $\begin{array}{l}\text { Caliche, soft, and } \\
\text { strips of sand........ }\end{array}$ & 7 & 107 \\
\hline $\begin{array}{l}\text { Caliche, hard.......... } \\
\text { Caliche, blocky, and }\end{array}$ & 4 & 111 \\
\hline very coarse gravel.... & 12 & 123 \\
\hline $\begin{array}{l}\text { Clay................... } \\
\text { Gravel, coarse, com- }\end{array}$ & 4 & 127 \\
\hline 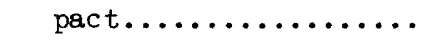 & 9 & 136 \\
\hline Gravel, coarse........ & 7 & 143 \\
\hline Sandstone............. & 7 & 150 \\
\hline 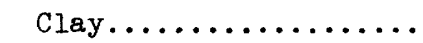 & 7 & 157 \\
\hline 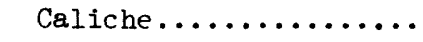 & 3 & 160 \\
\hline $\begin{array}{l}\text { Gravel, coarse, loose, } \\
\text { and soft clay......... }\end{array}$ & 11 & 171 \\
\hline $\begin{array}{l}\text { Clay and very coarse } \\
\text { gravel } \ldots \ldots \ldots \ldots \ldots\end{array}$ & 8 & 179 \\
\hline $\begin{array}{c}\text { Caliche and very coarse } \\
\text { gravel............ }\end{array}$ & 7 & 186 \\
\hline Clay, sandy.......... & 6 & 192 \\
\hline 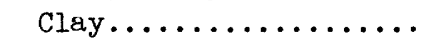 & 3 & 195 \\
\hline Caliche, hard......... & 3 & 198 \\
\hline Clay, sandy........... & 2 & 200 \\
\hline
\end{tabular}

Table 2.--Logs of test holes, seismograph shot holes, and wells--Continued

Kit Carson County--Continued

\begin{tabular}{l|c|c}
\hline & $\begin{array}{c}\text { Thickness } \\
\text { (feet) }\end{array}$ & $\begin{array}{c}\text { Depth } \\
\text { (feet) }\end{array}$ \\
\hline
\end{tabular}

C7-45-29ac--Continued

Ogallala formation--Con.

Gravel and strips of

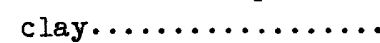

Gravel.

Clay, yellow, and

strips of white clay..

Clay, sandy...........

Gravel.............

Clay, white..........

Gravel, coarse.........

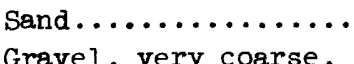

Gravel, very coarse,

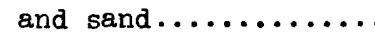

Gravel and clay.......

Gravel, very coarse,

and sand............

Gravel and clay........

Conglomerate, hard,

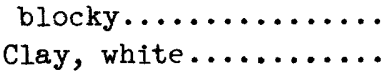

Gravel, fine, loose....

Clay, sandy............

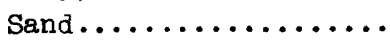

Clay.

Sand

Gravel.

Pierre shale(?):

Shele.....

C7-46-16dd

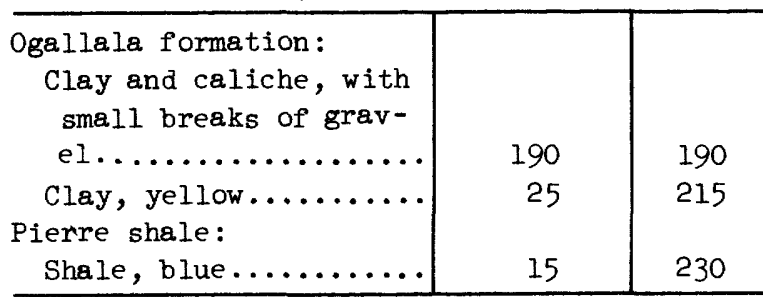

C7-47-24. (North half of section)

\begin{tabular}{|c|c|c|}
\hline 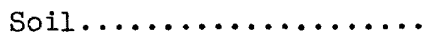 & 3 & 3 \\
\hline Ogallala formation: & & \\
\hline Clay and caliche....... & 165 & 168 \\
\hline Gravel............. & 22 & 190 \\
\hline Pierre shale: & & \\
\hline 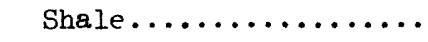 & $\cdots$ & 190 \\
\hline
\end{tabular}


Table 2.--Logs of test holes, seismograph shot holes, and wells--Continued

Kit Carson County--Continued

\begin{tabular}{|c|c|c|}
\hline & $\begin{array}{c}\text { Thickness } \\
\text { (feet) }\end{array}$ & $\begin{array}{l}\text { Depth } \\
\text { (feet) }\end{array}$ \\
\hline \multicolumn{3}{|c|}{$C 7-49-31 a$} \\
\hline 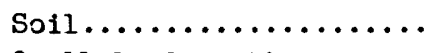 & 5 & 5 \\
\hline Ogallala formation: & & \\
\hline Clay and caliche....... & 174 & 179 \\
\hline Gravel (water).......... & 5 & 184 \\
\hline Pierre shale: & & \\
\hline 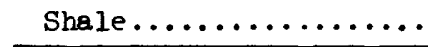 & ... & 184 \\
\hline
\end{tabular}

\begin{tabular}{|c|c|c|}
\hline \multicolumn{3}{|c|}{ C7-51-1aa } \\
\hline Ogallala formation: & & \\
\hline 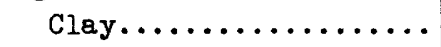 & 40 & 40 \\
\hline Gravel and sand........ & 110 & 150 \\
\hline Clay, yellow........... & 15 & 165 \\
\hline Pierre shale: & & \\
\hline Shale, blue $\ldots . . \ldots \ldots \ldots$ & 5 & 170 \\
\hline
\end{tabular}

\begin{tabular}{|c|c|c|}
\hline \multicolumn{3}{|c|}{$C 7-51-21 a a$} \\
\hline Ogallala formation: & & \\
\hline 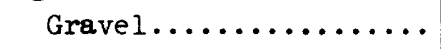 & 25 & 25 \\
\hline Sand $\ldots \ldots \ldots \ldots \ldots \ldots$ & 75 & 100 \\
\hline Caliche............. & 15 & 115 \\
\hline Clay, yellow........... & 15 & 130 \\
\hline Pierre shale: & & \\
\hline Shale, blue........... & 10 & 140 \\
\hline
\end{tabular}

C8-42-7dd. Altitude, 4,000 feet

\begin{tabular}{|c|c|c|}
\hline 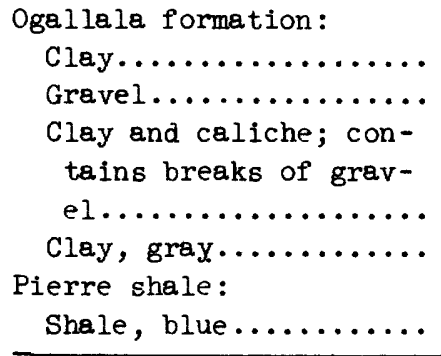 & $\begin{array}{r}220 \\
5\end{array}$ & $\begin{array}{l}320 \\
325 \\
340\end{array}$ \\
\hline C8-42-18ad. Altitu & & \\
\hline 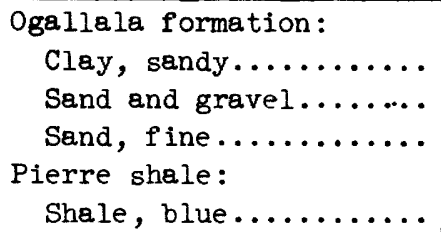 & $\begin{array}{r}50 \\
250 \\
40\end{array}$ & $\begin{array}{r}50 \\
300 \\
340\end{array}$ \\
\hline
\end{tabular}

Table 2.--Logs of test holes, seismograph shot holes, and wells--Continued

Kit Carson County--Continued

\begin{tabular}{l|c|c}
\hline & $\begin{array}{c}\text { Thickness } \\
\text { (feet) }\end{array}$ & $\begin{array}{c}\text { Depth } \\
\text { (feet) }\end{array}$ \\
\hline
\end{tabular}

c8-42-18cc. Altitude, 4,026 feet

\begin{tabular}{|c|c|c|}
\hline $\begin{array}{l}\text { Ogallala formation: } \\
\text { Clay.................. }\end{array}$ & 40 & 40 \\
\hline Gravel............. & 120 & 160 \\
\hline $\begin{array}{l}\text { Clay and caliche; con- } \\
\text { tains small breaks of } \\
\text { gravel.............. }\end{array}$ & 90 & 250 \\
\hline 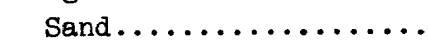 & 75 & 325 \\
\hline Pierre shale: & & \\
\hline Shale, blue........... & 15 & 340 \\
\hline
\end{tabular}

C8-42-18cd. Altitude, 4,024 feet

\begin{tabular}{|c|c|c|}
\hline \multicolumn{3}{|l|}{ Ogallala formation: } \\
\hline 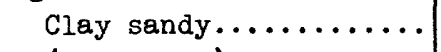 & 30 & 30 \\
\hline (No sample)............. & 15 & 45 \\
\hline Sand and gravel... & 155 & 200 \\
\hline 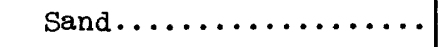 & 130 & 330 \\
\hline Pierre shale: & & \\
\hline Shale, blue........... & 20 & 350 \\
\hline
\end{tabular}

C8-42-18da. Altitude, 4,008 feet

\begin{tabular}{|c|c|c|}
\hline 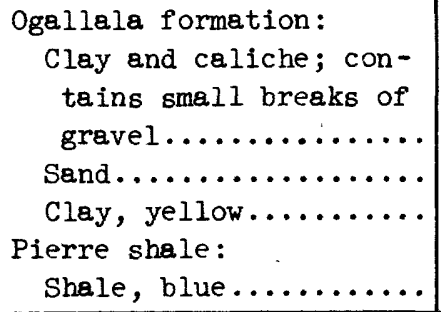 & $\begin{array}{r}250 \\
70 \\
15\end{array}$ & $\begin{array}{l}250 \\
320 \\
335 \\
340\end{array}$ \\
\hline C8-42-18dc. Altitud & 4,015 & \\
\hline 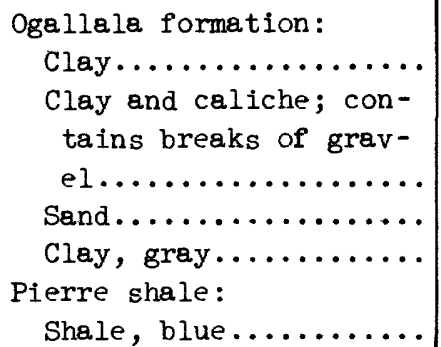 & $\begin{array}{r}200 \\
60 \\
20\end{array}$ & $\begin{array}{l}250 \\
310 \\
330\end{array}$ \\
\hline
\end{tabular}


Table 2.--Logs of test holes, seismograph shot holes, and wells--Continued

Kit Carson County--Continued

\begin{tabular}{c|c}
$\begin{array}{c}\text { Thickness } \\
\text { (feet) }\end{array}$ & $\begin{array}{c}\text { Depth } \\
\text { (feet) }\end{array}$ \\
\hline
\end{tabular}

c8-42-20aa. Altitude, 3,985 feet

\begin{tabular}{|c|c|c|}
\hline Ogallala formation: & & \\
\hline $\begin{array}{l}\text { Clay } \ldots \ldots \ldots \ldots \ldots \ldots \\
\text { Sand and caliche; con- } \\
\text { tains streaks of grav- }\end{array}$ & 60 & 60 \\
\hline 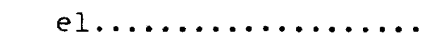 & 130 & 190 \\
\hline $\begin{array}{l}\text { Clay, containing strips } \\
\text { of sand............... }\end{array}$ & 90 & 280 \\
\hline $\begin{array}{l}\text { Pierre shale: } \\
\text { Shale, blue.......... }\end{array}$ & 20 & 300 \\
\hline
\end{tabular}

c8-42-20ab. Altitude, 3,987 feet

\begin{tabular}{|c|c|c|}
\hline \multirow{2}{*}{$\begin{array}{l}\text { Ogallala formation : } \\
\text { Clay, sandy........... }\end{array}$} & & \\
\hline & 55 & 55 \\
\hline Sand............... & 45 & 100 \\
\hline Sand and gravel....... & 160 & 260 \\
\hline Shale, gray......... & 20 & 280 \\
\hline Pierre shale: & & \\
\hline Shale, blue.......... & 20 & 300 \\
\hline
\end{tabular}

c8-42-20ba. Altitude, 3,995 feet

\begin{tabular}{|c|c|c|}
\hline Ogallala formation: & & \\
\hline 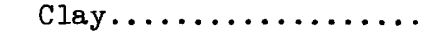 & 60 & 60 \\
\hline $\begin{array}{l}\text { Sand and gravel } \\
\text { streaks ............ }\end{array}$ & 140 & 200 \\
\hline 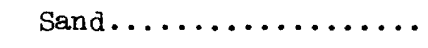 & 105 & .305 \\
\hline Pierre shale: & & \\
\hline Shale, blue.......... & $\cdot 15$ & 320 \\
\hline
\end{tabular}

C8-42-20bb. Altitude, 4,011 feet

\begin{tabular}{l|r|r}
\hline ogallala formation: & & \\
Clay, sandy........... & 50 & 50 \\
Sand and gravel....... & 280 & 330 \\
Pierre shale: & & \\
Shale, blue.......... & 20 & 350 \\
\hline
\end{tabular}

c8-42-20bc. Altitude, 4,001 feet

\begin{tabular}{|c|c|c|}
\hline $\begin{array}{l}\text { Ogallala formation: } \\
\text { Clay and caliche....... } \\
\text { Sand and gravel....... } \\
\text { Clay, yellow......... } \\
\text { Pierre shale: } \\
\text { Shale, blue.......... }\end{array}$ & $\begin{array}{r}70 \\
180 \\
15\end{array}$ & $\begin{array}{r}70 \\
250 \\
265\end{array}$ \\
\hline
\end{tabular}

Table 2.--Logs of test holes, seismograph shot holes, and wells--Continued

Kit Carson County--Continued

\begin{tabular}{|l|c|c}
\hline & $\begin{array}{c}\text { Thickness } \\
\text { (feet) }\end{array}$ & $\begin{array}{c}\text { Depth } \\
\text { (feet) }\end{array}$ \\
\hline
\end{tabular}

C8-42-20cb. Altitude, 3,999 feet

\begin{tabular}{|c|c|c|}
\hline Ogallala formation: & & \\
\hline Clay, sandy........... & 50 & 50 \\
\hline Sand and gravel....... & 150 & 200 \\
\hline Sand .............. & 50 & 250 \\
\hline Shale, gray.......... & 10 & 260 \\
\hline $\begin{array}{l}\text { Pierre shale: } \\
\text { Shale, blue........... }\end{array}$ & 20 & 280 \\
\hline
\end{tabular}

c8-42-20cc. Altitude, 3,996 feet

\begin{tabular}{|c|c|c|}
\hline Ogallala formation: & & \\
\hline 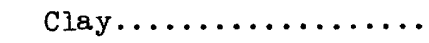 & 70 & 70 \\
\hline 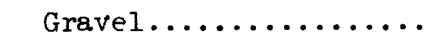 & 20 & 90 \\
\hline $\begin{array}{c}\text { Clay and caliche, with } \\
\text { breaks of sand......... }\end{array}$ & 160 & 250 \\
\hline Clay, yellow.......... & 15 & 265 \\
\hline Pierre shale: & & \\
\hline Shale, blue........... & 15 & 280 \\
\hline
\end{tabular}

\section{$\mathrm{c} 8-43-22 \mathrm{bb}$}

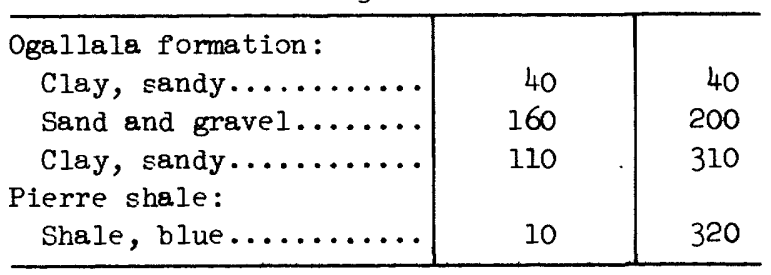

\section{C8 $-44-22 b b$}

\begin{tabular}{|c|c|c|}
\hline \multicolumn{3}{|l|}{ Ogallala formation: } \\
\hline Clay, sandy.......... & 50 & 50 \\
\hline Clay and caliche...... & 200 & 250 \\
\hline 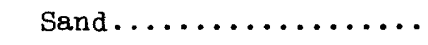 & 75 & 325 \\
\hline Pierre shale: & & \\
\hline Shale, blue.......... & 15 & 340 \\
\hline
\end{tabular}

C8-44-36cd. Drilled by K. G. Wilcox, 1948

\begin{tabular}{|c|c|c|}
\hline 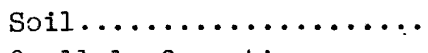 & 5 & 5 \\
\hline Ogallala formation: & & \\
\hline Clay, yellow......... & 31 & 36 \\
\hline $\begin{array}{l}\text { Caliche, soft.......... } \\
\text { Gravel, containing }\end{array}$ & 14 & 50 \\
\hline strips of hard rock... & 19 & 69 \\
\hline Caliche, hard......... & 4 & 73 \\
\hline
\end{tabular}


Table 2.--Logs of test holes, seismograph shot holes, and wells--Continued

Kit Carson County--Continued

\begin{tabular}{|c|c|c|}
\hline & $\begin{array}{c}\text { Thickness } \\
\text { (feet) }\end{array}$ & $\begin{array}{l}\text { Depth } \\
\text { (feet) }\end{array}$ \\
\hline \multicolumn{3}{|c|}{ c8-44-36cd--Continued } \\
\hline \multicolumn{3}{|l|}{ Ogallala formation--Con. } \\
\hline Clay, sandy $\ldots \ldots \ldots \ldots$ & 4 & 77 \\
\hline Caliche, hard......... & 1 & 78 \\
\hline Clay, sandy .......... & 8 & 86 \\
\hline Conglomerate......... & 8 & 94 \\
\hline \multicolumn{3}{|l|}{ Gravel, containing } \\
\hline strips of hard rock... & $\begin{array}{l}9 \\
6\end{array}$ & 103 \\
\hline 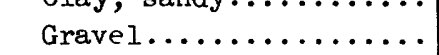 & 7 & 116 \\
\hline Clay, sandy.......... & 6 & 122 \\
\hline Conglomerate.......... & 9 & 131 \\
\hline \multicolumn{3}{|l|}{ Clay, containing strips } \\
\hline \multicolumn{3}{|l|}{ Caliche, hard, and } \\
\hline 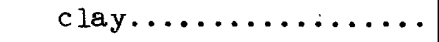 & 14 & 156 \\
\hline Caliche, hard......... & 8 & 164 \\
\hline Gravel, coarse........ & 6 & 170 \\
\hline Rock, hard........... & 4 & 174 \\
\hline \multicolumn{3}{|l|}{$\begin{array}{l}\text { Clay, sandy, containing } \\
\text { strips of hard rock... }\end{array}$} \\
\hline Conglomerate......... & 4 & 185 \\
\hline Caliche, hard........ & 15 & 200 \\
\hline Sand, fine........... & 2 & 202 \\
\hline Sandstone, hard....... & 5 & 207 \\
\hline Sand, fine........... & 1 & 208 \\
\hline Sandstone, hard....... & 1 & 209 \\
\hline Sand, fine.......... & 1 & 210 \\
\hline Sandstone, hard....... & 4 & 214 \\
\hline \multirow{2}{*}{\multicolumn{3}{|c|}{$\begin{array}{l}\text { Sandstone, containing } \\
\text { strips of clay and }\end{array}$}} \\
\hline & 10 & 224 \\
\hline Clay, brown.......... & 17 & 241 \\
\hline \multicolumn{3}{|l|}{ Clay, containing strips } \\
\hline Sand, fine ........... & 6 & 265 \\
\hline \multirow{2}{*}{\multicolumn{3}{|c|}{$\begin{array}{l}\text { Sand, fine, containing } \\
\text { strips of clay and } \\
\text { rock.............. }\end{array}$}} \\
\hline & 29 & 294 \\
\hline Joint clay, brown...... & 4 & 298 \\
\hline Sand, fine.......... & 7 & 305 \\
\hline \multicolumn{3}{|l|}{ Sand, fine, and strips } \\
\hline Gravel.............. & 15 & 336 \\
\hline $\begin{array}{l}\text { Joint clay and strips } \\
\text { of gravel } \ldots \ldots \ldots \ldots \ldots\end{array}$ & 11 & 347 \\
\hline \multicolumn{3}{|l|}{ Pierre shale: } \\
\hline Shale, blue.......... & 3 & 350 \\
\hline
\end{tabular}

Table 2.--Logs of test holes, seismograph shot holes, and wells--Continued

Kit Carson County--Continued

\begin{tabular}{l|c|c}
\hline & $\begin{array}{c}\text { Thickness } \\
\text { (feet) }\end{array}$ & $\begin{array}{c}\text { Depth } \\
\text { (feet) }\end{array}$ \\
\hline
\end{tabular}

C8-44-36db. Dug by Kelly Well Co. Inc., 1928

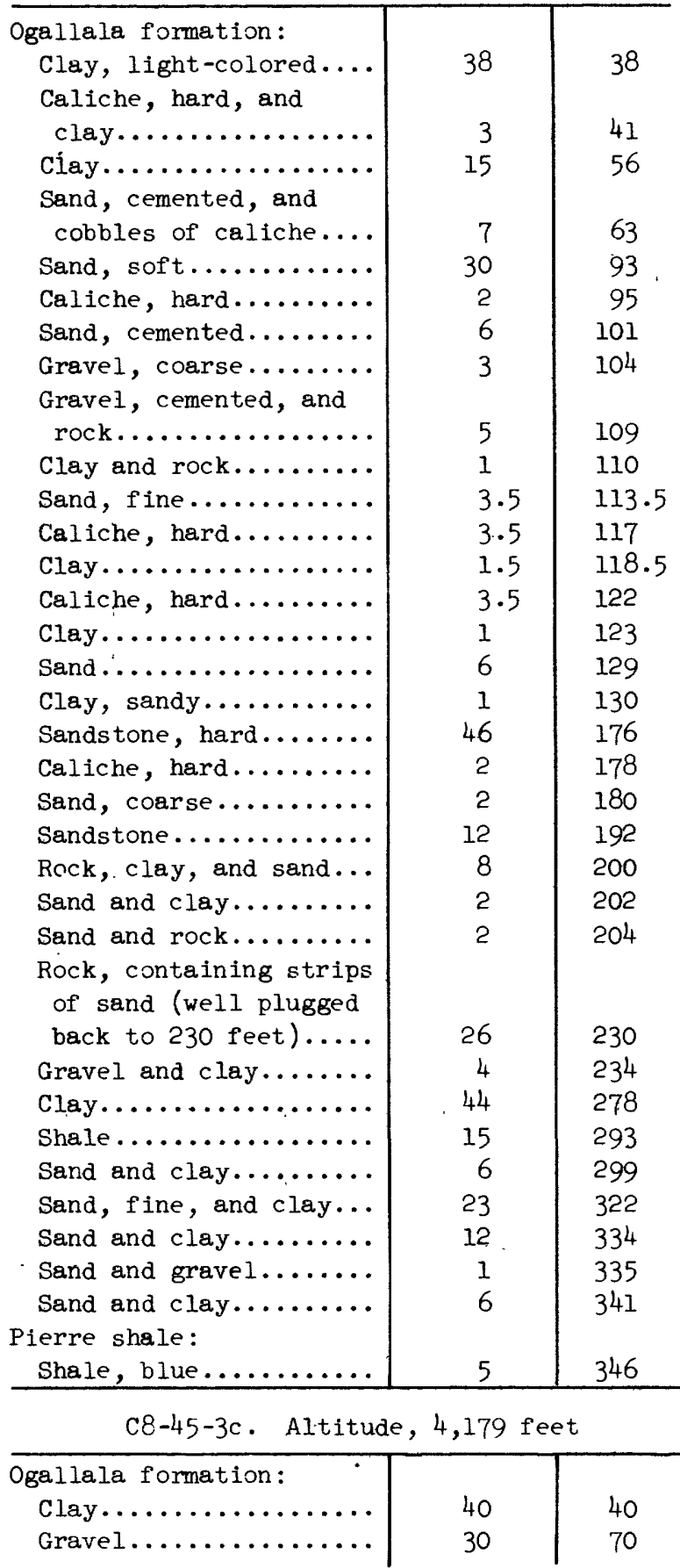


Table 2.--Logs of test holes, seismograph shot holes, and wells--Continued

Kit Carson County--Continued

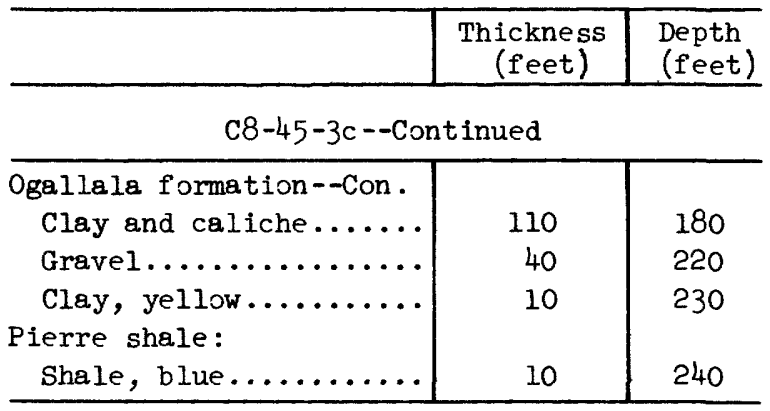

c8-45-4bb. Altitude, 4,232 feet

\begin{tabular}{|c|c|c|}
\hline \multicolumn{3}{|l|}{ Ogallala formation: } \\
\hline 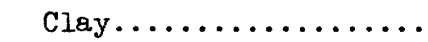 & 40 & 40 \\
\hline Gravel.............. & 30 & 70 \\
\hline Clay and caliche...... & 120 & 190 \\
\hline Gravel............. & 100 & 290 \\
\hline Clay, gray........... & 10 & 300 \\
\hline Pierre shale: & & \\
\hline Shale, blue........... & 10 & 310 \\
\hline
\end{tabular}

C8-45-5dd. Altitude, 4,170 feet

\begin{tabular}{|c|c|c|}
\hline Ogallala formation: & & \\
\hline 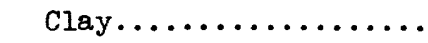 & 40 & 40 \\
\hline Gravel............ & 40 & 80 \\
\hline Clay and caliche...... & 80 & 160 \\
\hline Grave1............. & 40 & 200 \\
\hline Clay, gray........... & 10 & 210 \\
\hline Pierre shale: & & \\
\hline Shale, blue.......... & 10 & 220 \\
\hline
\end{tabular}

c8-45-6cc. Altitude, 4,215 feet

\begin{tabular}{|c|c|c|}
\hline \multicolumn{3}{|l|}{ Ogallala formation: } \\
\hline Sand................ & 30 & 30 \\
\hline Grave1 and sand....... & 40 & 70 \\
\hline Caliche........... & 20 & 90 \\
\hline Gravel.............. & 30 & 120 \\
\hline 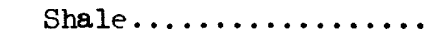 & 25 & 145 \\
\hline Sand and gravel....... & 45 & 190 \\
\hline Pierre shale: & & \\
\hline Shale, blue........... & $\cdots$ & 190 \\
\hline
\end{tabular}

C8-45-10cc. Altitude, 4,159 feet

\begin{tabular}{|c|c|c|}
\hline $\begin{array}{l}\text { Silt................. } \\
\text { Ogallala formation: }\end{array}$ & 30 & 30 \\
\hline Grave1............ & 40 & 70 \\
\hline $\begin{array}{l}\text { Sand and streaks of } \\
\text { caliche } \ldots \ldots \ldots \ldots \ldots\end{array}$ & 100 & 170 \\
\hline $\begin{array}{l}\text { Shale, gray........... } \\
\text { Pierre shale: }\end{array}$ & 15 & 185 \\
\hline Shale, blue.......... & 15 & 200 \\
\hline
\end{tabular}

Table 2.--Logs of test holes, seismograph shot holes, and wells--Continued

Kit Carson County--Continued

\begin{tabular}{l|c|c}
\hline & $\begin{array}{c}\text { Thickness } \\
\text { (feet) }\end{array}$ & $\begin{array}{c}\text { Depth } \\
\text { (feet) }\end{array}$ \\
\hline
\end{tabular}

c8-45-13cc. Altitude, 4,209 feet

\begin{tabular}{|c|c|c|}
\hline \multirow{2}{*}{ 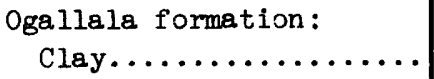 } & & \\
\hline & 40 & 40 \\
\hline Gravel............. & 50 & 90 \\
\hline Clay and caliche...... & 100 & 190 \\
\hline 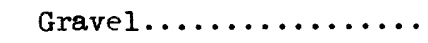 & 45 & 235 \\
\hline Clay, yellow......... & 10 & 245 \\
\hline Pierre shale: & & \\
\hline Shale, blue.......... & 5 & 250 \\
\hline
\end{tabular}

C8-45-13d. Altitude, 4,157 feet

\begin{tabular}{|c|c|c|}
\hline Ogallala formation: & & \\
\hline 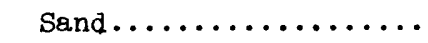 & 20 & 20 \\
\hline Grave1............. & 30 & 50 \\
\hline Caliche............ & 70 & 120 \\
\hline $\begin{array}{l}\text { Shale, sandy, and grav- } \\
\text { el............... }\end{array}$ & 60 & 180 \\
\hline Caliche............. & 40 & 220 \\
\hline $\begin{array}{l}\text { Pierre shale: } \\
\text { Shale, blue.......... }\end{array}$ & $\ldots$ & 220 \\
\hline
\end{tabular}

C8-45-14a. Altitude, 4,206 feet

\begin{tabular}{|c|c|c|}
\hline Oga1lala formation: & & \\
\hline 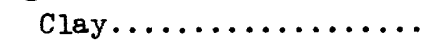 & 40 & 40 \\
\hline Gravel............. & 40 & 80 \\
\hline Clay and caliche...... & 100 & 180 \\
\hline Caliche............ & 55 & 235 \\
\hline Gravel............. & 10 & 245 \\
\hline Clay, yellow.......... & 5 & 250 \\
\hline Pierre shale: & & \\
\hline Shale, blue.......... & $\cdots$ & 250 \\
\hline
\end{tabular}

C8-45-15aa. Altitude, 4,175 feet

\begin{tabular}{|c|c|c|}
\hline 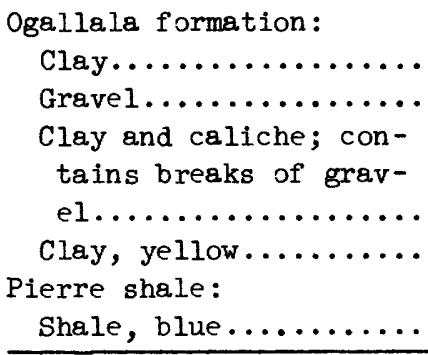 & $\begin{array}{r}5 \\
30 \\
\\
175 \\
10 \\
\ldots\end{array}$ & $\begin{array}{l}210 \\
220 \\
220\end{array}$ \\
\hline c8-45-15dd. Altitu & 4,219 & \\
\hline $\begin{array}{l}\text { Ogallala formation: } \\
\text { Clay, sandy.......... } \\
\text { Sand and gravel....... } \\
\text { Clay, sandy } \ldots \ldots \ldots \ldots\end{array}$ & $\begin{array}{r}35 \\
65 \\
150\end{array}$ & $\begin{array}{r}35 \\
100 \\
250\end{array}$ \\
\hline
\end{tabular}


Table 2.--Logs of test holes, seismograph shot holes, and wells--Continued

Kit Carson County---Continued

\begin{tabular}{|c|c|c|}
\hline & $\begin{array}{c}\text { Thickness } \\
\text { (feet) }\end{array}$ & $\begin{array}{l}\text { Depth } \\
\text { (feet) }\end{array}$ \\
\hline \multicolumn{3}{|c|}{ C8-45-15dd--Continued } \\
\hline $\begin{array}{l}\text { Pierre shale: } \\
\text { Shale, blue.......... }\end{array}$ & 10 & 260 \\
\hline \multicolumn{3}{|c|}{ c8-45-16cc. Altitude, 4,152 feet } \\
\hline $\begin{array}{l}\text { Ogallala formation: } \\
\text { Gravel.............. } \\
\text { Clay and caliche, with } \\
\text { breaks of gravel...... } \\
\text { Clay, yellow......... } \\
\text { Pierre shale: } \\
\text { Shale, blue.......... }\end{array}$ & $\begin{array}{r}15 \\
130 \\
13 \\
12\end{array}$ & $\begin{array}{r}15 \\
145 \\
158\end{array}$ \\
\hline \multicolumn{3}{|c|}{ c8-45-17cc. Altitude, 4,234 feet } \\
\hline 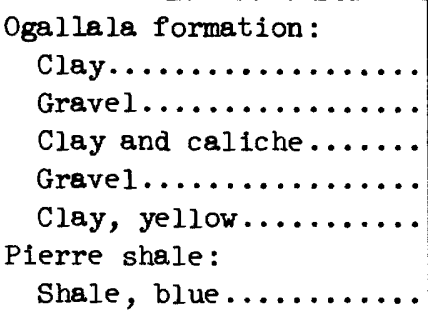 & $\begin{array}{r}15 \\
25 \\
120 \\
50 \\
10 \\
10\end{array}$ & $\begin{array}{r}15 \\
40 \\
160 \\
210 \\
220 \\
230\end{array}$ \\
\hline \multicolumn{3}{|c|}{ c8-45-2lcc. Altitude, 4,214 feet } \\
\hline 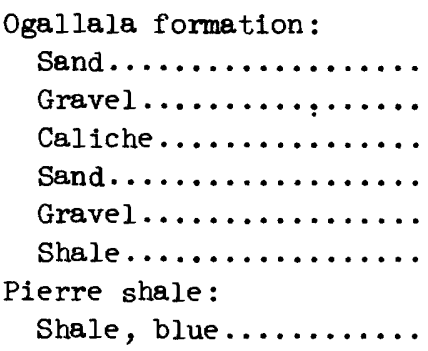 & $\begin{array}{r}30 \\
40 \\
22 \\
8 \\
80 \\
8 \\
12\end{array}$ & $\begin{array}{r}30 \\
70 \\
92 \\
100 \\
180 \\
188 \\
200\end{array}$ \\
\hline \multicolumn{3}{|c|}{ c8-45-22cc. Altitude, 4,197 feet } \\
\hline 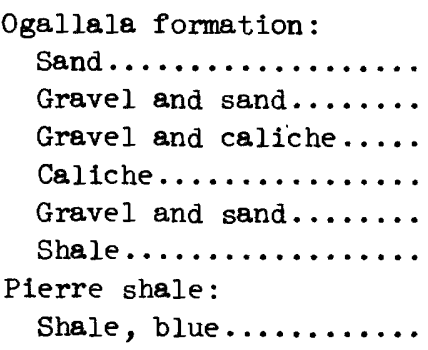 & $\begin{array}{l}30 \\
20 \\
30 \\
20 \\
75 \\
10 \\
15\end{array}$ & $\begin{array}{r}30 \\
50 \\
80 \\
100 \\
175 \\
185 \\
200\end{array}$ \\
\hline
\end{tabular}

Table 2.--Logs of test holes, seismograph shot holes, and wells--Continued

Kit Carson County--Continued

\begin{tabular}{|c|c|c|}
\hline & $\begin{array}{l}\text { Thickness } \\
\text { (feet) }\end{array}$ & $\begin{array}{l}\text { Depth } \\
\text { (feet) }\end{array}$ \\
\hline \multicolumn{3}{|c|}{ c8-45-26cc. Altitude, 4,232 feet } \\
\hline Ogallala formation: & & \\
\hline 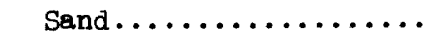 & 30 & 30 \\
\hline 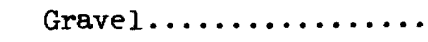 & 90 & 120 \\
\hline $\begin{array}{l}\text { Shale, sandy, and grav- } \\
\text { el............... }\end{array}$ & 120 & 240 \\
\hline Pierre shale: & & , . \\
\hline Shale, blue........... & 15 & 255 \\
\hline
\end{tabular}

\begin{tabular}{|c|c|c|}
\hline \multicolumn{3}{|l|}{ Ogallala formation: } \\
\hline Clву.............. & 30 & 30 \\
\hline Gravel............. & 30 & 60 \\
\hline Clay and caliche...... & 80 & 140 \\
\hline Gravel............. & 22 & 162 \\
\hline Clay, gray............ & 8 & 170 \\
\hline Pierre shale: & & \\
\hline Shale, blue.......... & 10 & 180 \\
\hline
\end{tabular}

C8-45-29bb. Altitude, 4,248 feet

\begin{tabular}{|c|c|c|}
\hline Ogallala formation: & & \\
\hline Sand............... & 20 & 20 \\
\hline Caliche............. & 40 & 60 \\
\hline Gravel............ & 40 & 100 \\
\hline $\begin{array}{c}\text { Gravel; contains cal1- } \\
\text { che breaks........... }\end{array}$ & 130 & 230 \\
\hline Pierre shale: & & \\
\hline Shale, blue........... & 10 & 240 \\
\hline
\end{tabular}

c8-45-34bb. Altitude, 4,245 feet

\begin{tabular}{|c|c|c|}
\hline \multicolumn{3}{|l|}{ Ogallala formation: } \\
\hline 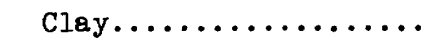 & 30 & 30 \\
\hline Gravel............. & 40 & 70 \\
\hline Clay and caliche...... & 160 & 230 \\
\hline Pierre shale: & & \\
\hline Shale, blue.......... & 10 & 240 \\
\hline
\end{tabular}

\begin{tabular}{|c|c|c|}
\hline \multicolumn{3}{|l|}{ Ogallala formetion: } \\
\hline 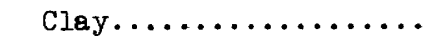 & 40 & 40 \\
\hline Grave1............ & 40 & 80 \\
\hline Clay and caliche...... & 90 & 170 \\
\hline Gravel............. & 65 & 235 \\
\hline Clay, yellow.......... & 15 & 250 \\
\hline Pierre shale: & & \\
\hline Shale, blue........... & 10 & 260 \\
\hline
\end{tabular}


Table 2.--Logs of test holes, seismograph shot holes, and wells--Continued

Kit Carson County--Continued

\begin{tabular}{l|c|c}
\hline & $\begin{array}{c}\text { Thickness } \\
\text { (feet) }\end{array}$ & $\begin{array}{c}\text { Depth } \\
\text { (feet) }\end{array}$ \\
\hline
\end{tabular}

C8-46-laa. Altitude, 4,247 feet

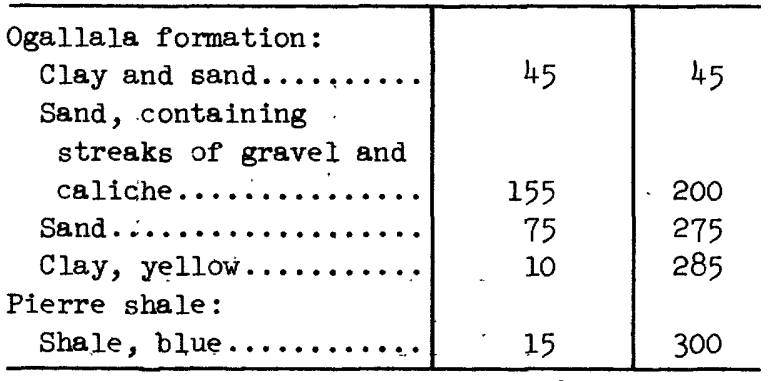

C8-46-1bb. Altitude, 4,284 feet

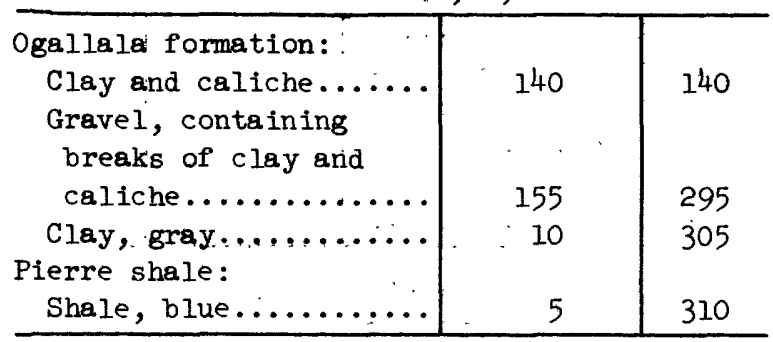

C8-46-3a. Altitude, 4,271 feet

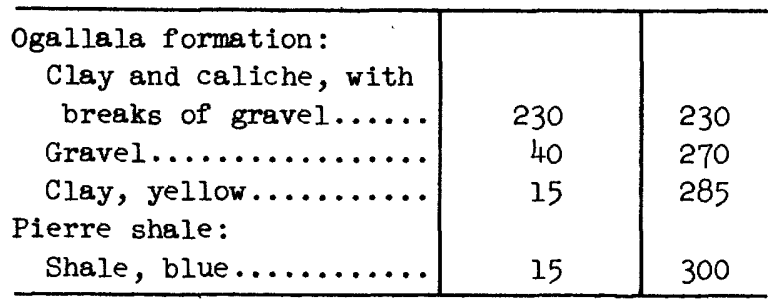

c8-46-3bb. Altitude, 4,290 feet

\begin{tabular}{|c|c|c|}
\hline $\begin{array}{l}\text { Ogallala formation: } \\
\text { Clay } \ldots \ldots \ldots \ldots \ldots \ldots\end{array}$ & 30 & 30 \\
\hline $\begin{array}{l}\text { Clay and caliche, with } \\
\text { breaks of gravel...... }\end{array}$ & 250 & 280 \\
\hline Clay, yellow.......... & 10 & 290 \\
\hline $\begin{array}{l}\text { Pierre shale: } \\
\text { Shale, blue........... }\end{array}$ & 10 & 300 \\
\hline
\end{tabular}

c8-46-1laa. Altitude, 4,277 feet

\begin{tabular}{|c|c|c|}
\hline Clay .............. & 35 & 35 \\
\hline 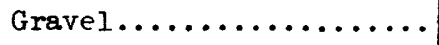 & 35 & 70 \\
\hline Clay and caliche........ & 120 & 190 \\
\hline (No sample) ............. & 90 & 280 \\
\hline $\begin{array}{l}\text { Clay, gray } . . . \cdots \cdots \cdots \cdots \\
\text { Pierre shale: }\end{array}$ & 10 & 290 \\
\hline Shale, blue.......... & 10 & 300 \\
\hline
\end{tabular}

Table 2.--Logs of test holes, seismograph shot holes, and wells--Continued

Kit Carson County--Continued

\begin{tabular}{l|c|c}
\hline & $\begin{array}{c}\text { Thickness } \\
\text { (feet) }\end{array}$ & $\begin{array}{c}\text { Depth } \\
\text { (feet) }\end{array}$ \\
\hline \multicolumn{2}{c}{ c8-46-13aa. Altitude, 4,276 feet } \\
\hline $\begin{array}{c}\text { Ogallala formation: } \\
\text { Sand and gravel } \\
\text { streaks............. }\end{array}$ & 90 & 90 \\
$\begin{array}{c}\text { Sand and gravel; con- } \\
\text { tains streaks of cali- } \\
\text { che............... }\end{array}$ & 110 & 200 \\
Sand and gravel....... & 75 & 275 \\
Pierre shale: & & \\
Shale, blue........... & 15 & 290 \\
\hline
\end{tabular}

c8-46-13bb. Altitude, 4,262 feet.

\begin{tabular}{|c|c|c|}
\hline 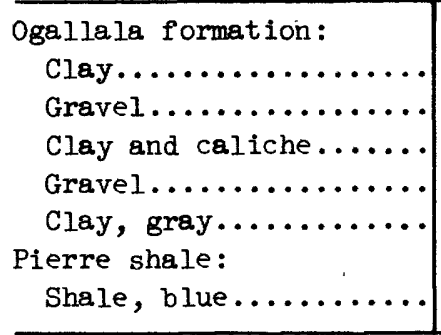 & $\begin{array}{r}28 \\
32 \\
130 \\
55 \\
5\end{array}$ & $\begin{array}{r}28 \\
60 \\
190 \\
245 \\
250 \\
260\end{array}$ \\
\hline
\end{tabular}

C8-46-15b. Altitude, 4,306 feet

\begin{tabular}{|c|c|c|}
\hline \multicolumn{3}{|l|}{ Ogallala formation: } \\
\hline Clay and caliche...... & 150 & 150 \\
\hline Gravel.............. & 45 & 195 \\
\hline Clay and caliche....... & 55 & 250 \\
\hline Clay, yellow.......... & 10 & 260 \\
\hline Pierre shale: & & \\
\hline Shale, blue........... & 10 & 270 \\
\hline
\end{tabular}

C8-46-15c. Altitude, 4,316 feet

\begin{tabular}{l|r|r}
\hline Ogallala formation: & & \\
Clay, with breaks of & & \\
gravel and caliche.... & 230 & 230 \\
Sand............... & 30 & 260 \\
Pierre shale: & & \\
Shale, blue........... & 10 & 270 \\
\hline
\end{tabular}

C8-46-18dd. Altitude, 4,355 reet

\begin{tabular}{|c|c|c|}
\hline Ogallala formation: & & \\
\hline $\begin{array}{c}\text { Clay and caliche, with } \\
\text { breaks of gravel...... }\end{array}$ & 260 & 260 \\
\hline Clay, yellow........... & 10 & 270 \\
\hline $\begin{array}{l}\text { Pierre shale: } \\
\text { Shale, blue............ }\end{array}$ & 10 & 280 \\
\hline
\end{tabular}


Table 2.--Logs of test holes, seismograph shot holes, and wells--Continued

\section{K1t Carson County--Continued}

\begin{tabular}{l|c|c}
\hline & $\begin{array}{c}\text { Thickness } \\
\text { (feet) }\end{array}$ & $\begin{array}{c}\text { Depth } \\
\text { (feet) }\end{array}$ \\
\hline
\end{tabular}

C8-46-22a. Altitude, 4,287 feet

\begin{tabular}{|c|c|c|}
\hline Ogallala formation: & & \\
\hline Clay...$\ldots \ldots \ldots \ldots$ & 8 & 8 \\
\hline Gravel.............. & 22 & 30 \\
\hline Clay and caliche....... & 150 & 180 \\
\hline (No sample) $\ldots \ldots \ldots \ldots$ & 45 & 225 \\
\hline Clay, yellow........... & 5 & 230 \\
\hline Pierre shale: & & \\
\hline Shale, blue............ & 10 & 240 \\
\hline
\end{tabular}

C8-46-22c. Altitude, 4,337 feet

\begin{tabular}{|c|c|c|}
\hline Ogallala formation: & & \\
\hline Clay, sandy $\ldots \ldots \ldots \ldots$ & 60 & $\begin{array}{r}60 \\
130\end{array}$ \\
\hline $\begin{array}{l}\text { Gravel and sand } \ldots \ldots \ldots \\
\text { Caliche................ }\end{array}$ & 20 & $\begin{array}{l}130 \\
150\end{array}$ \\
\hline Sand $\ldots \ldots \ldots \ldots \ldots$ & 80 & 230 \\
\hline Clay, yellow.......... & 10 & 240 \\
\hline Pierre shale: & & \\
\hline Shale, blue. & 10 & 250 \\
\hline
\end{tabular}

C8-46-22dd. Altitude, 4,325 feet

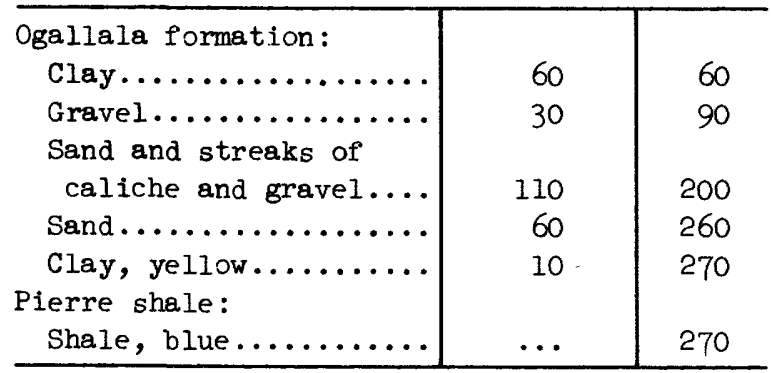

C8-46-24bb. Altitude, 4,268 feet

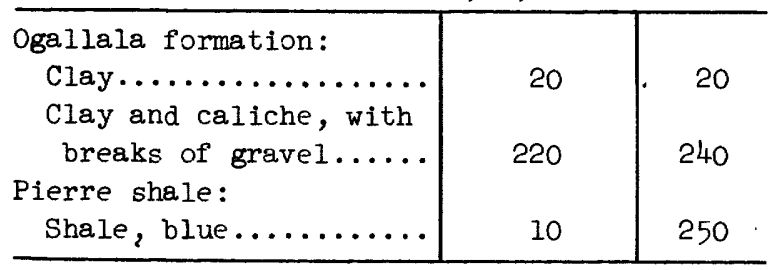

C8-46-25aa. Altitude, 4,248 feet

\begin{tabular}{|c|c|c|}
\hline Ogallala formation: & & \\
\hline 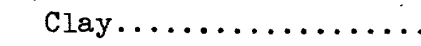 & 20 & 20 \\
\hline Gravel............... & 25 & 45 \\
\hline Clay and caliche....... & 115 & 160 \\
\hline Gravel............... & 40 & 200 \\
\hline Clay, gray $\ldots \ldots \ldots \ldots \ldots$ & 15 & 215 \\
\hline
\end{tabular}

Table 2.--Logs of test holes, seismograph shot holes, and wells--Continued

Kit Carson County--Continued

\begin{tabular}{l|c|c}
\hline & $\begin{array}{c}\text { Thickness } \\
\text { (feet) }\end{array}$ & $\begin{array}{c}\text { Depth } \\
\text { (feet) }\end{array}$ \\
\hline
\end{tabular}

C8-46-25aa--6ontinued

\begin{tabular}{r|c|c}
\hline $\begin{array}{l}\text { Pierre shale: } \\
\text { Shale, blue............. }\end{array}$ & 15 & 230 \\
\hline
\end{tabular}

C8-46-25bb. Altitude, 4,323 feet

\begin{tabular}{|c|c|c|}
\hline Ogallala formation: & & \\
\hline 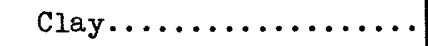 & 40 & 40 \\
\hline Gravel.............. & 30 & 70 \\
\hline Clay and caliche....... & 155 & 225 \\
\hline Gravel $\ldots \ldots \ldots \ldots \ldots \ldots$ & 40 & 265 \\
\hline Pierre shale: & & \\
\hline Shale, blue... & 15 & 280 \\
\hline
\end{tabular}

C8-46-25c. Altitude, 4,324 feet

\begin{tabular}{|c|c|c|}
\hline \multicolumn{3}{|l|}{ Ogallala formation: } \\
\hline Clay...$\ldots \ldots \ldots \ldots$ & 30 & 30 \\
\hline Gravel.............. & 28 & 58 \\
\hline $\begin{array}{c}\text { Clay and caliche, with } \\
\text { breaks of gravel....... }\end{array}$ & 112 & 170 \\
\hline 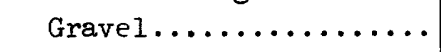 & 80 & 250 \\
\hline 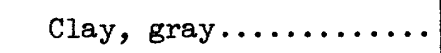 & 10 & 260 \\
\hline Pierre shale: & & \\
\hline Shale, blue........... & 10 & 270 \\
\hline
\end{tabular}

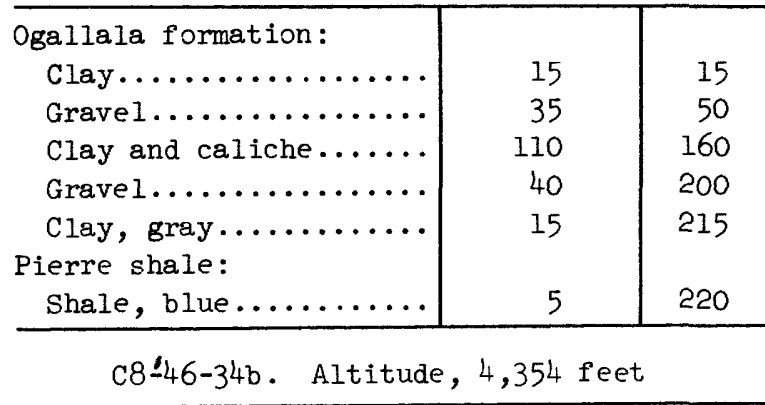

\begin{tabular}{|c|c|c|}
\hline \multirow{2}{*}{ Silt ................... } & 10 & 10 \\
\hline & 20 & 30 \\
\hline Sand and gravel....... & 65 & 95 \\
\hline Clay, sandy........... & 40 & 135 \\
\hline $\begin{array}{l}\text { Sand and gravel; con- } \\
\text { tains small breaks of } \\
\text { caliche.............. }\end{array}$ & 65 & 200 \\
\hline 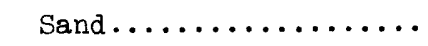 & 60 & 260 \\
\hline Pierre shale: & & \\
\hline Shale, blue........... & 10 & 270 \\
\hline
\end{tabular}


Table 2.--Logs of test holes, seismograph shot holes, and wells--Continued

Kit Carson County--Continued

\begin{tabular}{l|c|c}
\hline & $\begin{array}{c}\text { Thickness } \\
\text { (feet) }\end{array}$ & $\begin{array}{c}\text { Depth } \\
\text { (feet) }\end{array}$ \\
\hline
\end{tabular}

c8-46-35bb. Altitude, 4,319 feet

\begin{tabular}{|c|c|c|}
\hline 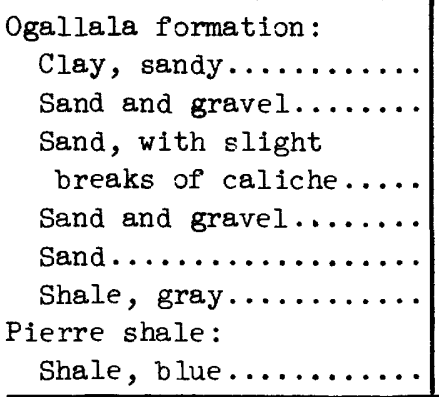 & $\begin{array}{l}40 \\
65 \\
30 \\
10\end{array}$ & $\begin{array}{r}40 \\
95 \\
135 \\
200 \\
230 \\
240\end{array}$ \\
\hline
\end{tabular}

\section{c8-47-1c}

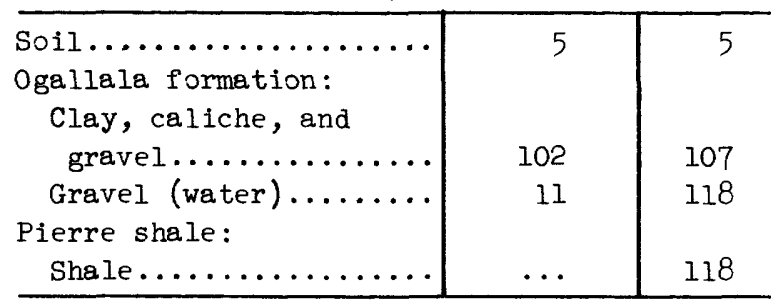

\section{C8-47-4aa}

\begin{tabular}{|c|c|c|}
\hline 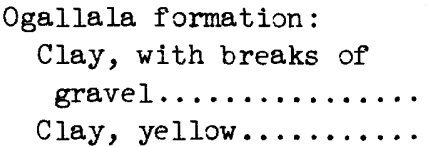 & $\begin{array}{r}270 \\
20\end{array}$ & $\begin{array}{l}270 \\
290\end{array}$ \\
\hline Pierre shale: & 70 & 300 \\
\hline
\end{tabular}

C8-47-10aa

\begin{tabular}{|c|c|c|}
\hline \multicolumn{3}{|l|}{ Ogallala formation: } \\
\hline 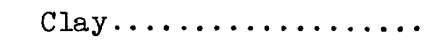 & 10 & 10 \\
\hline Gravel............ & 10 & 20 \\
\hline 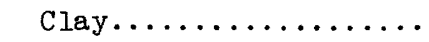 & 10 & 30 \\
\hline $\begin{array}{l}\text { Sand and streaks of } \\
\text { sandy shale......... }\end{array}$ & 90 & 120 \\
\hline Clay, yellow......... & 25 & 145 \\
\hline $\begin{array}{l}\text { Pierre shale: } \\
\text { Shale, blue........... }\end{array}$ & 15 & 160 \\
\hline
\end{tabular}

\section{C8-47-11aa}

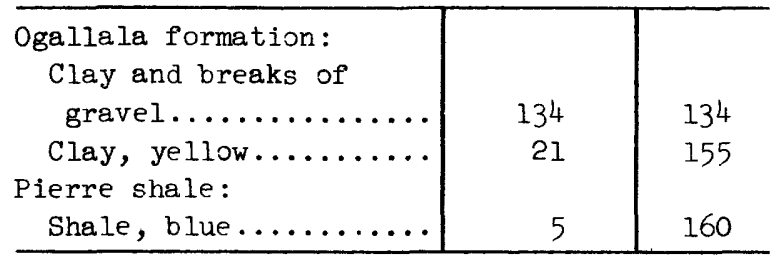

Table 2.--Logs of test holes, seismograph shot holes, and wells--Continued

Kit Carson County--Continued

\begin{tabular}{l|c|c}
\hline & $\begin{array}{c}\text { Thickness } \\
\text { (feet) }\end{array}$ & $\begin{array}{c}\text { Depth } \\
\text { (feet) }\end{array}$ \\
\hline
\end{tabular}

$$
\text { C8-47-14dd }
$$

\begin{tabular}{|c|c|c|}
\hline \multicolumn{3}{|l|}{ Ogallala formation: } \\
\hline 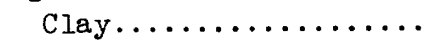 & 30 & 30 \\
\hline Gravel.............. & 10 & 40 \\
\hline Sand.............. & 50 & 90 \\
\hline Gravel and sand....... & 30 & 120 \\
\hline Shale, sandy......... & $1 \dot{5}$ & 135 \\
\hline Clay, yellow......... & 23 & 158 \\
\hline Pierre shale: & & \\
\hline Shale, blue. & 2 & 160 \\
\hline
\end{tabular}

\section{c8-47-17aa}

\begin{tabular}{|c|c|c|}
\hline Ogallala formation: & & \\
\hline 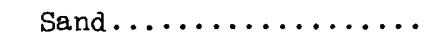 & 105 & 105 \\
\hline Shale, sandy......... & 15 & 120 \\
\hline Clay, yellow......... & 20 & 140 \\
\hline Shale, gray......... & 13 & 153 \\
\hline Pierre shale: & & \\
\hline Shale, blue......... & 7 & 160 \\
\hline
\end{tabular}

\section{C8-47-19dd}

\begin{tabular}{|c|c|c|}
\hline \multicolumn{3}{|l|}{ Ogallala formation: } \\
\hline Sand and gravel....... & 50 & 50 \\
\hline Clay and caliche...... & 60 & 110 \\
\hline Clay, yellow......... & 23 & 133 \\
\hline Pierre shale: & & \\
\hline Shale, blue.......... & 7 & 140 \\
\hline
\end{tabular}

\section{C8-47-21dd}

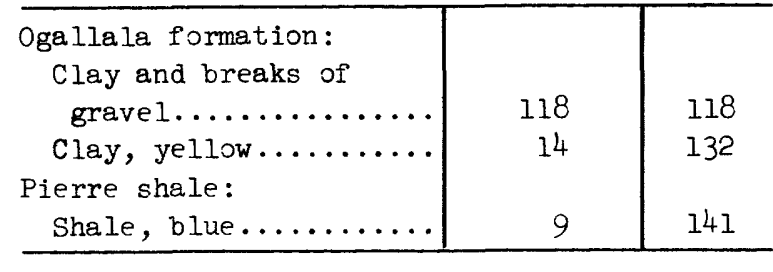

$c 8-47-26 b b$

\begin{tabular}{|c|c|c|}
\hline \multicolumn{3}{|l|}{ Ogallala formation: } \\
\hline 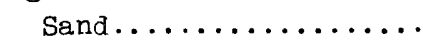 & 50 & 50 \\
\hline Shale, sandy......... & 20 & 70 \\
\hline Sand and gravel...... & 40 & 110 \\
\hline 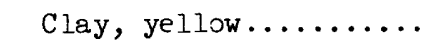 & 10 & 120 \\
\hline Pierre shale: & & \\
\hline Shale, blue.......... & 10 & 130 \\
\hline
\end{tabular}


Table 2.--Logs of test holes, seismograph shot holes, and wells--Continued

Kit Carson County--Continued

\begin{tabular}{|c|c|c|}
\hline & $\begin{array}{c}\text { Thickness } \\
\text { (feet) }\end{array}$ & $\begin{array}{l}\text { Depth } \\
\text { (feet) }\end{array}$ \\
\hline \multicolumn{3}{|c|}{$c 8-47-26 c$} \\
\hline $\begin{array}{l}\text { Ogallala formation: } \\
\text { Sand, gravel, and } \\
\text { caliche............. }\end{array}$ & 60 & 60 \\
\hline 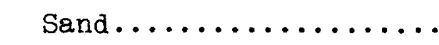 & 30 & 90 \\
\hline Clay, yellow.......... & 15 & 105 \\
\hline Pierre shale: & & \\
\hline Shale, blue........... & 15 & 120 \\
\hline
\end{tabular}

c8 $8-47-28 c c$

\begin{tabular}{|c|c|c|}
\hline \multicolumn{3}{|l|}{ Ogallala formation: } \\
\hline 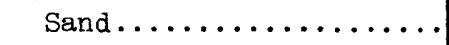 & 70 & 70 \\
\hline 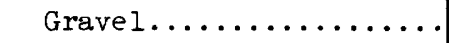 & 15 & 85 \\
\hline 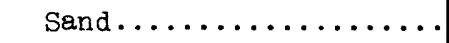 & 25 & 110 \\
\hline Shale, sandy........... & 10 & 120 \\
\hline Clay, yellow........... & 20 & 140 \\
\hline Pierre shale: & & \\
\hline Shale, blue........... & 10 & 150 \\
\hline
\end{tabular}

\begin{tabular}{|c|c|c|}
\hline \multicolumn{3}{|c|}{$c 8-47-28 d$} \\
\hline $\begin{array}{l}\text { Ogallala formation: } \\
\text { Clay and sand......... } \\
\text { Clay, yellow.......... } \\
\text { Pierre shale: } \\
\text { Shale, blue } \ldots \ldots \ldots \ldots\end{array}$ & $\begin{array}{r}120 \\
12\end{array}$ & $\begin{array}{l}120 \\
132 \\
141\end{array}$ \\
\hline \multicolumn{3}{|c|}{$c 8-48-24 a a$} \\
\hline $\begin{array}{l}\text { Ogallala formation: } \\
\text { Sand and sandy shale... } \\
\text { Clay, yellow, and gray } \\
\text { shale............... } \\
\text { Pierre shale: } \\
\text { Shale, blue........... }\end{array}$ & $\begin{array}{l}109 \\
21 \\
10\end{array}$ & $\begin{array}{l}109 \\
130 \\
140\end{array}$ \\
\hline
\end{tabular}

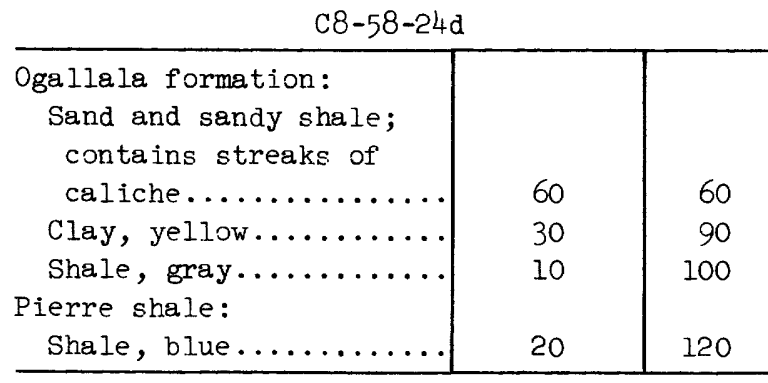

Table 2.--Logs of test holes, seismograph shot holes, and wells--Continued

Kit Carson County--Continued

\begin{tabular}{|c|c|c|}
\hline & $\begin{array}{c}\text { Thickness } \\
\text { (feet) }\end{array}$ & $\begin{array}{l}\text { Depth } \\
\text { (feet) }\end{array}$ \\
\hline \multicolumn{3}{|c|}{$c 8-48-28 a a$} \\
\hline 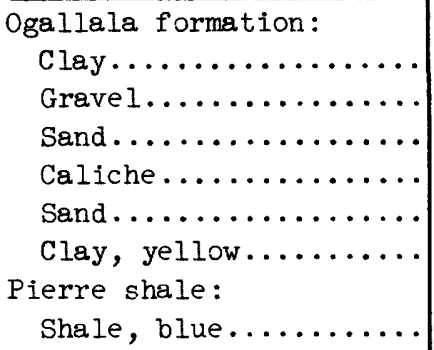 & $\begin{array}{l}20 \\
20 \\
70 \\
10 \\
20 \\
20 \\
10\end{array}$ & $\begin{array}{r}20 \\
40 \\
110 \\
120 \\
140 \\
160 \\
170\end{array}$ \\
\hline \multicolumn{3}{|c|}{$c 8-48-36 a a$} \\
\hline $\begin{array}{l}\text { Ogallala formation: } \\
\text { Sand and gravel....... } \\
\text { Caliche............ } \\
\text { Shale, gray......... } \\
\text { Pierre shale: } \\
\text { Shale, blue.......... }\end{array}$ & $\begin{array}{l}55 \\
35 \\
20\end{array}$ & $\begin{array}{r}55 \\
90 \\
110 \\
130\end{array}$ \\
\hline
\end{tabular}

$c 8-48-36 d$

\begin{tabular}{|c|c|c|}
\hline \\
\hline 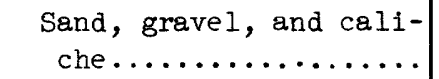 & 80 & 80 \\
\hline Gravel.............. & 10 & 90 \\
\hline Sand............... & 20 & 110 \\
\hline Gravel............. & 10 & 120 \\
\hline 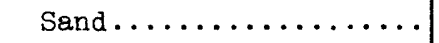 & 20 & 140 \\
\hline Clay, yellow.......... & 20 & 160 \\
\hline Pierre shale: & & \\
\hline Shale, blue.......... & 10 & 170 \\
\hline
\end{tabular}

\section{C8-49-2laa}

\begin{tabular}{|c|c|c|}
\hline \multicolumn{3}{|l|}{ Ogallala formation: } \\
\hline 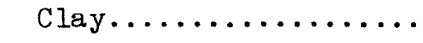 & 27 & 27 \\
\hline Sand and gravel....... & 33 & 60 \\
\hline Clay, yellow.......... & 30 & 90 \\
\hline Pierre shale: & & \\
\hline Shale, blue.......... & 20 & 110 \\
\hline
\end{tabular}

\begin{tabular}{|c|c|c|}
\hline \multicolumn{3}{|c|}{ C8-50-1aa } \\
\hline Ogallala formation: & & \\
\hline 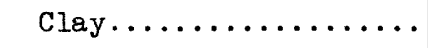 & 20 & 20 \\
\hline Gravel............ & 28 & 48 \\
\hline Clay and caliche...... & 52 & 100 \\
\hline Clay, yellow......... & 40 & 140 \\
\hline Pierre shale: & & \\
\hline Shale, blue......... & 10 & 150 \\
\hline
\end{tabular}


Table 2.--Logs of test holes, seismograph shot holes, and wells--Continued

Kit Carson County--Continued

\begin{tabular}{l|c|c}
\hline & $\begin{array}{c}\text { Thickness } \\
\text { (feet) }\end{array}$ & $\begin{array}{c}\text { Depth } \\
\text { (feet) }\end{array}$ \\
\hline
\end{tabular}

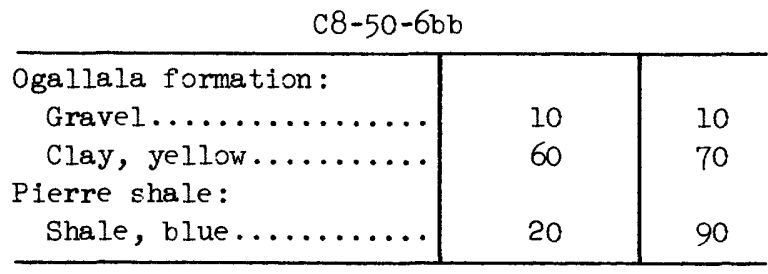

\begin{tabular}{|c|c|c|}
\hline \multicolumn{3}{|c|}{ C8-50-21aa } \\
\hline Ogallala formation: & & \\
\hline 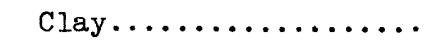 & 40 & 40 \\
\hline Gravel............ & 5 & 45 \\
\hline Shale, yellow......... & 75 & 120 \\
\hline Pierre shale: & & \\
\hline Shale, blue.......... & 30 & 150 \\
\hline \multicolumn{3}{|c|}{$c 8-51-6 b b$} \\
\hline
\end{tabular}

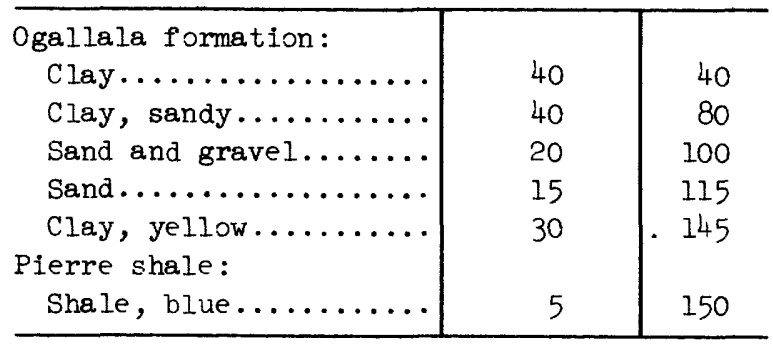

\section{$c 8-51-22 b b$}

\begin{tabular}{|c|c|c|}
\hline \multicolumn{3}{|l|}{ Ogallala formation: } \\
\hline 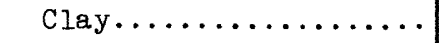 & 10 & 10 \\
\hline Gravel............ & 50 & 60 \\
\hline Clay, yellow and gray.. & 35 & 95 \\
\hline Pierre shale: & & \\
\hline Shale, blue.......... & 5 & 100 \\
\hline
\end{tabular}

C9-42-14dd. Altitude, 3,993 feet

\begin{tabular}{|c|c|c|}
\hline \multicolumn{3}{|l|}{ Ogallala formation: } \\
\hline 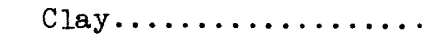 & 20 & 20 \\
\hline Gravel............ & 60 & 80 \\
\hline Clay and caliche...... & 60 & 140 \\
\hline Sand and grave $1 . \ldots \ldots$ & 175 & 315 \\
\hline Pierre shale: & & \\
\hline Shale, blue...... & 15 & 330 \\
\hline
\end{tabular}

C9-42-16dd. Altitude, 4,004 feet

\begin{tabular}{|c|c|c|}
\hline Ogallala formation: & & \\
\hline 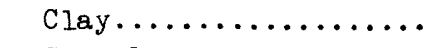 & 20 & 20 \\
\hline Grave1............ & 40 & 60 \\
\hline
\end{tabular}

Trble 2.--Logs of test holes, seismograph shot holes, and wells--Continued

Kit Carson County--Continued

\begin{tabular}{l|c|c}
\hline & $\begin{array}{c}\text { Thickness } \\
\text { (feet) }\end{array}$ & $\begin{array}{c}\text { Depth } \\
\text { (feet) }\end{array}$ \\
\hline
\end{tabular}

C9-42-16dd--Continued

\begin{tabular}{|c|c|c|}
\hline 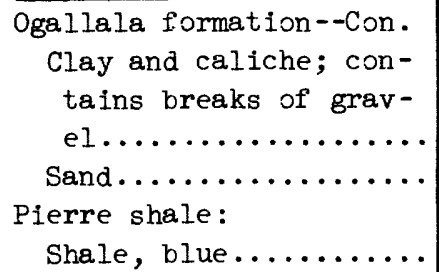 & $\begin{array}{r}180 \\
45 \\
15\end{array}$ & $\begin{array}{l}240 \\
285 \\
300\end{array}$ \\
\hline
\end{tabular}

C9-42-19dd. Altitude, 4,064 feet

\begin{tabular}{|c|c|c|}
\hline Ogallala formation: & & \\
\hline 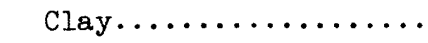 & 30 & 30 \\
\hline Sand and gravel....... & 270 & 300 \\
\hline Clay, yellow......... & 10 & 310 \\
\hline Pierre shale: & & \\
\hline Shale, blue........ & 10 & 320 \\
\hline
\end{tabular}

C9-43-8dd. Altitude, 4,099 feet

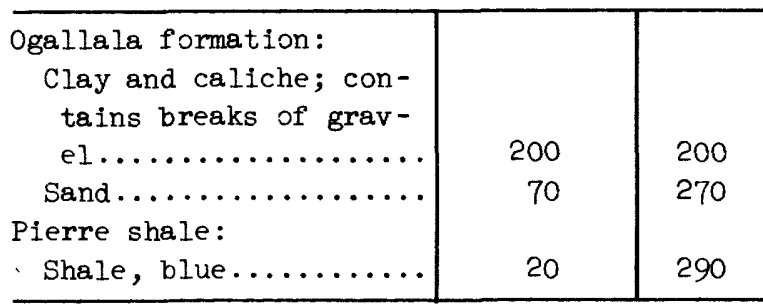

$$
c 9-43-16 c d
$$

\begin{tabular}{|c|c|c|}
\hline 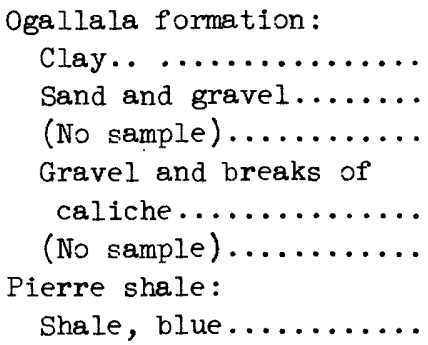 & $\begin{array}{l}50 \\
50 \\
30 \\
70 \\
80\end{array}$ & $\begin{array}{r}50 \\
100 \\
130 \\
200 \\
280\end{array}$ \\
\hline
\end{tabular}

$c 9-43-16 \mathrm{dc}$

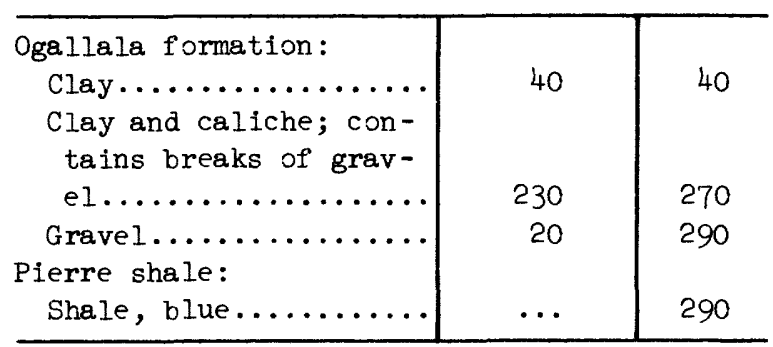


Table 2.--Logs of test holes, seismograph shot holes, and wells--Continued

Kit Carson County--Continued

\begin{tabular}{l|c|c}
\hline & $\begin{array}{c}\text { Thickness } \\
\text { (feet) }\end{array}$ & $\begin{array}{c}\text { Depth } \\
\text { (feet) }\end{array}$ \\
\hline
\end{tabular}

C9-43-17ad. Altitude, 4,086 feet

\begin{tabular}{|c|c|c|}
\hline Soil, sandy ........... & 25 & 25 \\
\hline Ogallala formation: & & \\
\hline Sand and gravel....... & 185 & 210 \\
\hline Sand, fine........... & 50 & 260 \\
\hline Shale, gray $\ldots \ldots \ldots \ldots$ & 10 & 270 \\
\hline Pierre shale: & & \\
\hline Shale, blue.......... & 20 & 290 \\
\hline
\end{tabular}

C9-43-17da. Altitude, 4,082 feet

\begin{tabular}{|c|c|c|}
\hline \\
\hline Ogallala formation: & & \\
\hline Gravel............. & 20 & 20 \\
\hline Clay and caliche...... & 40 & 60 \\
\hline Grave1............. & 30 & 90 \\
\hline $\begin{array}{l}\text { Clay and caliche; con- } \\
\text { tains breaks of grav- }\end{array}$ & & \\
\hline 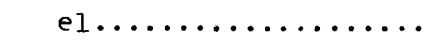 & 130 & 220 \\
\hline Sand............ & 30 & 250 \\
\hline Clay, gray........... & 10 & 260 \\
\hline Pierre shale: & & \\
\hline Shale, blue........... & 20 & 280 \\
\hline
\end{tabular}

c9-43-17dd. Altitude, 4,087 feet

\begin{tabular}{l|r|c}
\hline Soil, sandy............ & 25 & 25 \\
Ogallala formation. & & \\
Sand and gravel....... & 175 & 200 \\
Sand, fine.......... & 70 & 270 \\
Pierre shale. & & \\
Shale, blue......... & 20 & 290 \\
\hline
\end{tabular}

C9-43-20ad. Altitude, 4,097 feet

\begin{tabular}{|c|c|c|}
\hline $\begin{array}{l}\text { Soil and clay........... } \\
\text { Ogallala formation: }\end{array}$ & 25 & 25 \\
\hline Sand and gravel........ & 185 & 210 \\
\hline Sand, fine.......... & 50 & 260 \\
\hline Shale, gray.......... & 10 & 270 \\
\hline Pierre shale: & & \\
\hline Shale, blue.......... & 20 & 290 \\
\hline
\end{tabular}

C9-43-20da. Altitude, 4,134 feet

\begin{tabular}{|c|c|c|}
\hline \multicolumn{3}{|l|}{ Ogallala formation: } \\
\hline Clay and caliche...... & 70 & 70 \\
\hline 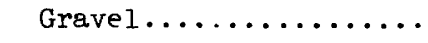 & 50 & 120 \\
\hline $\begin{array}{l}\text { Clay and caliche; con- } \\
\text { tains breaks of grav- }\end{array}$ & & \\
\hline 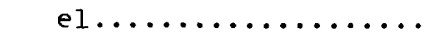 & 170 & 290 \\
\hline
\end{tabular}

Table 2.--Logs of test holes, seismograph shot holes, and wells--Continued

Kit Carson County--Continued

\begin{tabular}{l|c|c}
\hline & $\begin{array}{c}\text { Thickness } \\
\text { (feet) }\end{array}$ & $\begin{array}{c}\text { Depth } \\
\text { (feet) }\end{array}$ \\
\hline
\end{tabular}

C9-43-20da--Continued

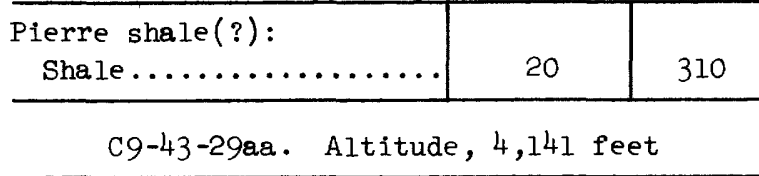

\begin{tabular}{|c|c|c|}
\hline \multicolumn{3}{|l|}{ Ogallala formation: } \\
\hline 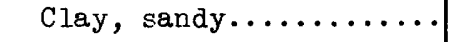 & 55 & 55 \\
\hline Sand and gravel........ & 255 & 310 \\
\hline Pierre shale: & & \\
\hline Shale, blue ............ & 10 & 320 \\
\hline
\end{tabular}

c9-43-29ad. Altitude, 4,165 feet

\begin{tabular}{|c|c|c|}
\hline \multirow{2}{*}{\multicolumn{3}{|c|}{ Ogallala formation: }} \\
\hline & & \\
\hline Clay, sandy........... & 60 & 60 \\
\hline 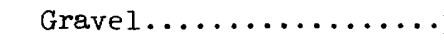 & 10 & 70 \\
\hline Clay, sandy.......... & 130 & 200 \\
\hline Sand and gravel...... & 140 & 340 \\
\hline Pierre shale: & & \\
\hline Shale, blue.......... & 10 & 350 \\
\hline
\end{tabular}

C9-43-29da. Altitude 4,168 feet

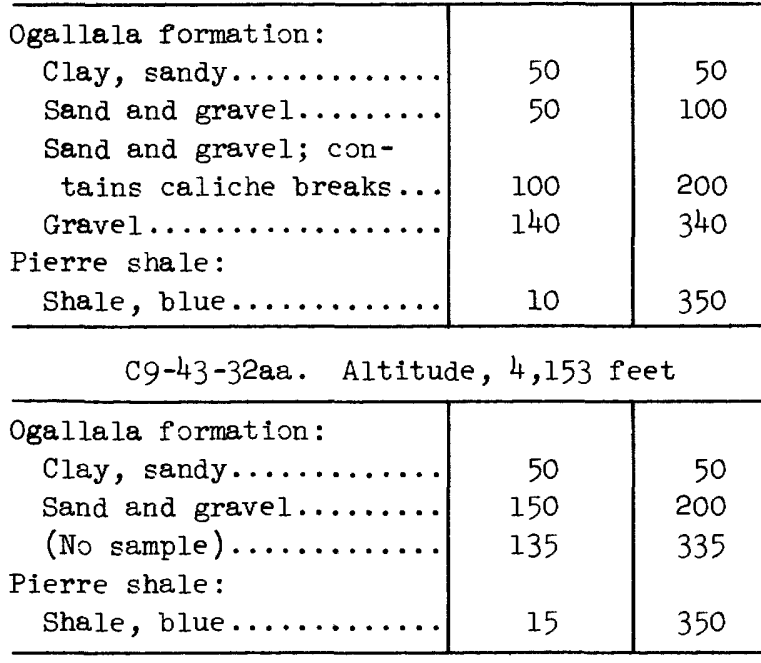

c9-43-32ad. Altitude, 4,148 feet

\begin{tabular}{|c|c|c|}
\hline \multicolumn{3}{|l|}{ Ogallala formation: } \\
\hline 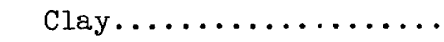 & 50 & 50 \\
\hline $\begin{array}{l}\text { Sand and gravel; con- } \\
\text { tains small breaks of } \\
\text { caliche and clay....... }\end{array}$ & 230 & 280 \\
\hline 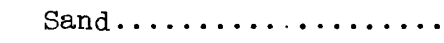 & 30 & 310 \\
\hline Pierre shale: & & \\
\hline Shale, blue......... & 50 & 360 \\
\hline
\end{tabular}


Table 2.--Logs of test holes, seismograph shot holes, and wells--Continued

Kit Carson County--Continued

\begin{tabular}{l|c|c}
\hline & $\begin{array}{c}\text { Thickness } \\
\text { (feet) }\end{array}$ & $\begin{array}{c}\text { Depth } \\
\text { (feet) }\end{array}$ \\
\hline
\end{tabular}

C9-43-32da. Altitude, 4,158 feet

Ogallala formation:

Clay and caliche......

Gravel and sand........

Caliche and gravel....

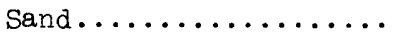

Pierre shale:

Shale, blue..........

\begin{tabular}{r|r}
55 & 55 \\
65 & 120 \\
35 & 155 \\
145 & 300 \\
10 & 310 \\
\hline
\end{tabular}

c9-43-32dd. Altitude, 4,148 feet

\begin{tabular}{|c|c|c|}
\hline Ogallala formation: & 55 & 5 \\
\hline $\begin{array}{l}\text { Gravel and sand........ } \\
\text { Sand and gravel }\end{array}$ & 145 & 200 \\
\hline streaks........... & 70 & 270 \\
\hline $\begin{array}{l}\text { Shale, sandy } . \ldots \ldots \ldots \ldots \\
\text { Pierre shale: }\end{array}$ & 25 & 295 \\
\hline Shale, blue.......... & 15 & 310 \\
\hline
\end{tabular}

c9-44-1a

\begin{tabular}{|c|c|c|}
\hline $\begin{array}{l}\text { Ogallala formation: } \\
\text { Clay.................. } \\
\text { Gravel, with small } \\
\text { breaks of clay....... } \\
\text { Pierre shale: } \\
\text { Shale, blue........... }\end{array}$ & $\begin{array}{r}74 \\
226\end{array}$ & $\begin{array}{r}74 \\
300\end{array}$ \\
\hline
\end{tabular}

c9-44-24aa

\begin{tabular}{|c|c|c|}
\hline \multicolumn{3}{|l|}{ Ogallala formation: } \\
\hline 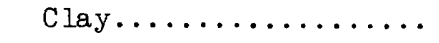 & 50 & 50 \\
\hline 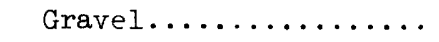 & 30 & 80 \\
\hline Clay and caliche...... & 145 & 225 \\
\hline 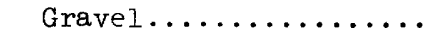 & 95 & 320 \\
\hline Clay, gray........... & 10 & 330 \\
\hline Pierre shale: & & \\
\hline Shale, blue.......... & 10 & 340 \\
\hline
\end{tabular}

C9-44-33da. Altitude, 4,221 feet

\begin{tabular}{|c|c|c|}
\hline \multicolumn{3}{|l|}{ Ogallala formation: } \\
\hline 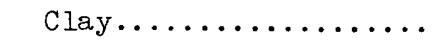 & 25 & 25 \\
\hline Gravel............. & 40 & 65 \\
\hline 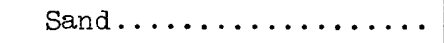 & 45 & 110 \\
\hline Caliche............. & 10 & 120 \\
\hline Sand and gravel....... & 70 & 190 \\
\hline Shale, sandy......... & 10 & 200 \\
\hline
\end{tabular}

Table 2.--Logs of test holes, seismograph shot holes, and wells--Continued

Kit Carson County--Continued

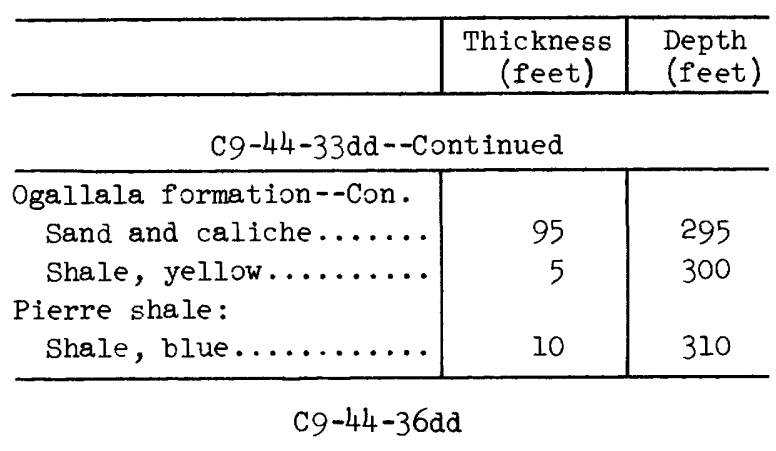

\begin{tabular}{|c|c|c|}
\hline \multirow{2}{*}{ 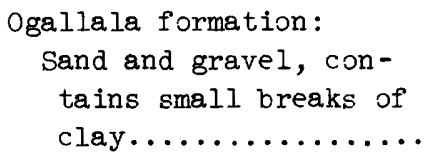 } & & \\
\hline & 315 & 315 \\
\hline Clay, gray........... & 5 & 320 \\
\hline Pierre shale: & & \\
\hline Shale, blue.......... & .. & 320 \\
\hline
\end{tabular}

\begin{tabular}{l|r|r}
\hline Ogallala formation: & & \\
Clay, caliche, gravel, & & \\
and breaks of sand.... & 142 & 142 \\
Clay, yellow........ & 11 & 153 \\
Pierre shale: & & \\
Shale, blue.......... & 7 & 160 \\
\hline
\end{tabular}

C9-45-13dd. Altitude, 4,270 feet

\begin{tabular}{|c|c|c|}
\hline \multicolumn{3}{|l|}{ Ogallala formation: } \\
\hline 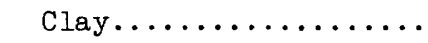 & 20 & 20 \\
\hline Gravel............ & 40 & 60 \\
\hline Clay and caliche...... & 55 & 115 \\
\hline (No sample)........... & 210 & 325 \\
\hline Pierre shale: & & \\
\hline Shale, blue.......... & 5 & 330 \\
\hline
\end{tabular}

C9-45-16ad. Altitude, 4,309 feet

\begin{tabular}{|c|c|c|}
\hline 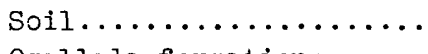 & 20 & 20 \\
\hline Ogallala formation: & & \\
\hline $\begin{array}{l}\text { Gravel.............. } \\
\text { Clay and caliche; con- } \\
\text { tains breaks of grav- }\end{array}$ & 20 & 40 \\
\hline el.............. & 240 & 280 \\
\hline 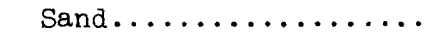 & 15 & 295 \\
\hline Pierre shale: & & \\
\hline Shale, blue.......... & 15 & 310 \\
\hline
\end{tabular}


Table 2.--Logs of test holes, seismograph shot holes, and wells--Continued

Kit Carson County--Continued

\begin{tabular}{l|c|c}
\hline & $\begin{array}{c}\text { Thickness } \\
\text { (feet) }\end{array}$ & $\begin{array}{c}\text { Depth } \\
\text { (feet) }\end{array}$ \\
\hline
\end{tabular}

C9-45-18cc. Altitude, 4,282 feet

\begin{tabular}{|c|c|c|}
\hline Ogallala formation: & & \\
\hline 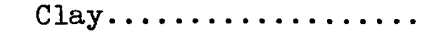 & 5 & 5 \\
\hline Gravel............. & 23 & 28 \\
\hline Clay and caliche........ & 72 & 100 \\
\hline Sand and gravel........ & 90 & 190 \\
\hline 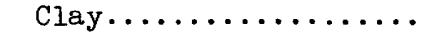 & 25 & 215 \\
\hline Pierre shale: & & \\
\hline Shale, blue...... & 5 & 220 \\
\hline
\end{tabular}

C9-45-23a. Altitude, 4,278 feet

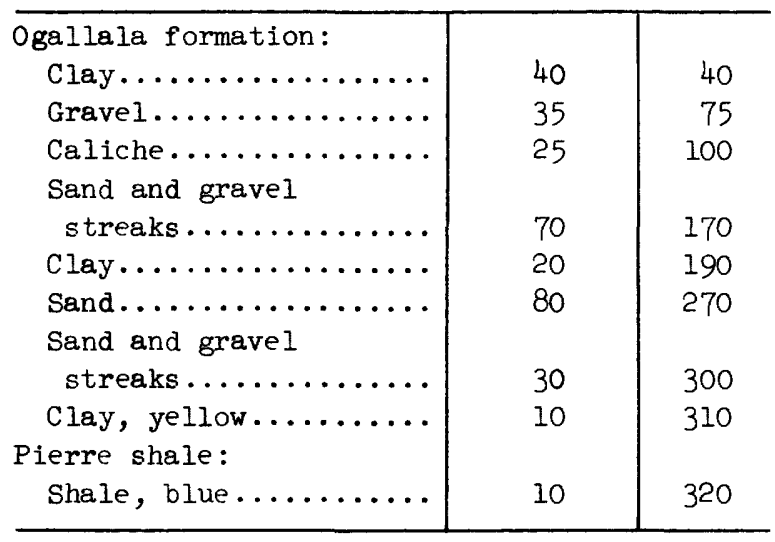

C9-45-33dd. Altitude, 4,303 feet

\begin{tabular}{|c|c|c|}
\hline \multicolumn{3}{|l|}{ Ogallala formation: } \\
\hline $\begin{array}{l}\text { Sand, gravel, and } \\
\text { caliche............. }\end{array}$ & 60 & 60 \\
\hline Shale, sandy.......... & 40 & 100 \\
\hline Caliche.............. & 20 & 120 \\
\hline $\operatorname{Sand} . \ldots \ldots \ldots \ldots \ldots$ & 40 & 160 \\
\hline Shale, sandy.......... & 40 & 200 \\
\hline Sand $\ldots \ldots \ldots \ldots \ldots \ldots$ & 55 & 255 \\
\hline Clay, yellow........... & 15. & 270 \\
\hline \multicolumn{3}{|l|}{ Pierre shale: } \\
\hline shale, blue.. & 10 & 280 \\
\hline
\end{tabular}

C9-46-31cb. Altitude, 4,394 feet

\begin{tabular}{|c|c|c|}
\hline \multicolumn{3}{|l|}{ Ogallala formation: } \\
\hline Caliche.....$\ldots \ldots$ & 70 & 90 \\
\hline 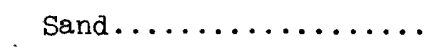 & 10 & 100 \\
\hline Sand and gravel........ & 30 & 130 \\
\hline Shale, gray........... & 13 & 143 \\
\hline Pierre shale: & & \\
\hline Shale, blue........... & 7 & 150 \\
\hline
\end{tabular}

Table 2.--Logs of test holes, seismograph shot holes, and wells--Continued

Kit Carson County--Continued

\begin{tabular}{l|c|c}
\hline & $\begin{array}{c}\text { Thickness } \\
\text { (feet) }\end{array}$ & $\begin{array}{c}\text { Depth } \\
\text { (feet) }\end{array}$ \\
\hline
\end{tabular}

c9-46-34cc. Altitúde, 4,352 feet

\begin{tabular}{|c|c|c|}
\hline 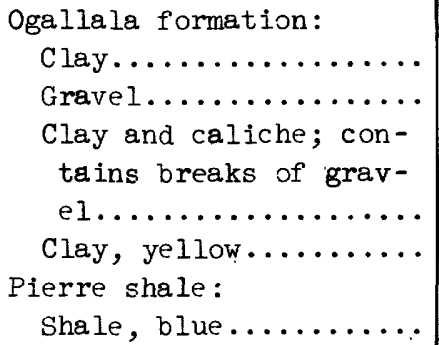 & $\begin{array}{r}126 \\
12\end{array}$ & $\begin{array}{l}170 \\
182\end{array}$ \\
\hline
\end{tabular}

C9-46-36d. Altitude, 4,364 feet

\begin{tabular}{|c|c|c|}
\hline & & \\
\hline Ogallala formation: & & \\
\hline Sand and gravel........ & 50 & 50 \\
\hline Shale, sandy .......... & 70 & 120 \\
\hline Sand $\ldots \ldots \ldots \ldots \ldots \ldots$ & 100 & 220 \\
\hline Clay, yellow.......... & 20 & 240 \\
\hline Pierre shale: & & \\
\hline Shale, blue............ & ... & 240 \\
\hline
\end{tabular}

C9-47-1ba. Drilled by K. G. Wilcox, 1949

\begin{tabular}{|c|c|c|}
\hline Soil.................. & 18 & 18 \\
\hline Ogallala formation: & & \\
\hline Rock, soft............ & 11 & 29 \\
\hline Rock, soft, and gravel. & 8 & 37 \\
\hline Rock, soft............ & 3 & 40 \\
\hline Rock and sand........... & 5 & 45 \\
\hline Caliche............... & 5 & 50 \\
\hline Caliche, sandy........ & 4 & 54 \\
\hline Sand and gravel; con- & & \\
\hline tains strips of rock.. & 1 & $\begin{array}{l}55 \\
57\end{array}$ \\
\hline Rock, hard............ & 2 & 57 \\
\hline 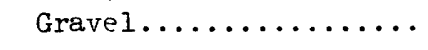 & 8 & 65 \\
\hline Gravel, coarse......... & 3 & 68 \\
\hline Sandstone..$\ldots \ldots \ldots \ldots$ & 1 & 69 \\
\hline Clay, sandy $\ldots \ldots \ldots \ldots$ & 1 & 70 \\
\hline Sandstone..$\ldots \ldots \ldots \ldots$ & 3 & 73 \\
\hline Gravel.............. & 4 & 77 \\
\hline Caliche, hard........... & 5 & 82 \\
\hline Clay and caliche....... & 23 & 105 \\
\hline Sandstone $\ldots \ldots \ldots \ldots \ldots$ & 4 & 109 \\
\hline$(?) \ldots \ldots \ldots \ldots \ldots \ldots \ldots$ & 1 & 110 \\
\hline Caliche, soft.......... & 10 & 120 \\
\hline Rock $\ldots \ldots \ldots \ldots \ldots \ldots$ & 17 & 137 \\
\hline Gravel............. & 18 & 155 \\
\hline Clay, sandy $\ldots \ldots \ldots \ldots$ & 3 & 158 \\
\hline Sand $\ldots \ldots \ldots \ldots \ldots \ldots$ & 2 & 160 \\
\hline Conglomerate...$\ldots \ldots \ldots$ & 3 & 163 \\
\hline
\end{tabular}


Table 2.--Logs of test holes, seismograph shot holes, and wells--Continued

Kit Carson County--Continued

\begin{tabular}{l|c|c}
\hline & $\begin{array}{c}\text { Thickness } \\
\text { (feet) }\end{array}$ & $\begin{array}{c}\text { Depth } \\
\text { (feet) }\end{array}$ \\
\hline
\end{tabular}

C9-47-1ba--Continued

\begin{tabular}{|c|c|c|}
\hline \multirow{2}{*}{\multicolumn{3}{|c|}{$\begin{array}{l}\text { Ogalla la formation--Con. } \\
\text { Sand and clay......... }\end{array}$}} \\
\hline & 4 & 167 \\
\hline 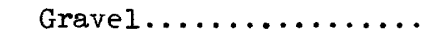 & 2 & 169 \\
\hline Clay................ & 4 & 173 \\
\hline Conglomerate......... & 1 & 174 \\
\hline Gravel............ & 1 & 175 \\
\hline Cong lomerate......... & 15 & 190 \\
\hline $\begin{array}{l}\text { Clay, white, containing } \\
\text { strips of gravel...... }\end{array}$ & 4 & 194 \\
\hline Sand and gravel....... & 13 & 207 \\
\hline Gravel, coarse........ & 2 & 209 \\
\hline Conglomerate......... & 12 & 221 \\
\hline Gravel............ & 13 & 234 \\
\hline Clay............... & 5 & 239 \\
\hline Grave1............. & 5 & 244 \\
\hline Pierre shale: & & \\
\hline Shale............. & 1 & 245 \\
\hline
\end{tabular}

C9-47-3b

\begin{tabular}{l|r|r}
\hline Ogallala formation: & & \\
Clay and gravel....... & 109 & 109 \\
Clay, yellow........ & 11 & 120 \\
Pierre shale: & & \\
Shale, blue......... & 10 & 130 \\
\hline
\end{tabular}

c9 $-47-5 b b$

\begin{tabular}{|c|c|c|}
\hline $\begin{array}{l}\text { Ogallala formation : } \\
\text { Clay and caliche....... }\end{array}$ & 40 & 40 \\
\hline 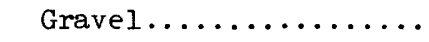 & 110 & 150 \\
\hline Clay, yellow.......... & 10 & 160 \\
\hline Pierre shale: & & \\
\hline Shale, blue.......... & 10 & 170 \\
\hline
\end{tabular}

c9-47-6dd

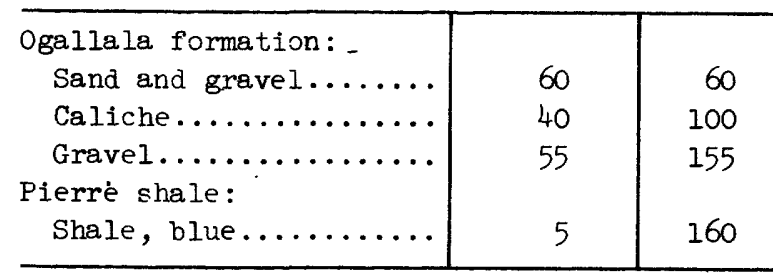

\section{C9-47-7dd}

Ogallala formation:

Sand and gravel.......

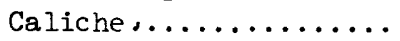

Gravel..............
Table 2.--Logs of test holes, seismograph shot holes, and wells--Continued

Kit Carson County--Continued

\begin{tabular}{l|c|c}
\hline & $\begin{array}{c}\text { Thickness } \\
\text { (feet) }\end{array}$ & $\begin{array}{c}\text { Depth } \\
\text { (feet) }\end{array}$ \\
\hline
\end{tabular}

c9-47-7dd--Cont inued

\begin{tabular}{l|l|l}
\hline $\begin{array}{l}\text { Pierre shale: } \\
\text { Shale, blue............... }\end{array}$ & 5 & 140 \\
\hline
\end{tabular}

\begin{tabular}{|c|c|c|}
\hline \multicolumn{3}{|c|}{$c 9-47-9 b b$} \\
\hline Ogallala formation: & & \\
\hline 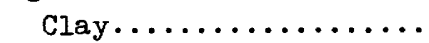 & 50 & 50 \\
\hline Clay and gravel....... & 70 & 120 \\
\hline Clay, yellow.......... & 17 & 137 \\
\hline Pierre shale: & & \\
\hline Shale, blue.......... & 3 & 140 \\
\hline
\end{tabular}

\begin{tabular}{|c|c|c|}
\hline \multicolumn{3}{|l|}{$c 9-47-9 c$} \\
\hline Ogallala formation: & & \\
\hline 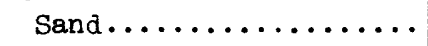 & 65 & 65 \\
\hline Gravel............ & 5 & 70 \\
\hline 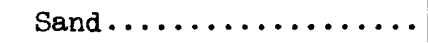 & 20 & 90 \\
\hline 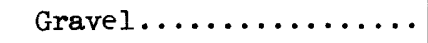 & 55 & 145 \\
\hline Shale, gray.......... & 7 & 152 \\
\hline Pierre shale: & & \\
\hline Shale, blue.......... & 8 & 160 \\
\hline
\end{tabular}

\begin{tabular}{|c|c|c|}
\hline \multicolumn{3}{|c|}{ C9-47-17dd } \\
\hline Ogallala formation: & & \\
\hline 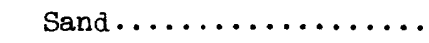 & 70 & 70 \\
\hline 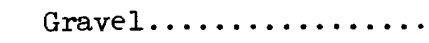 & 5 & 75 \\
\hline Sand and caliche...... & 25 & 100 \\
\hline Shale, sandy......... & 10 & 110 \\
\hline Clay, yellow......... & 40 & 150 \\
\hline Pierre shale: & & \\
\hline Shale, blue.......... & 10 & 160 \\
\hline
\end{tabular}

\begin{tabular}{|c|c|c|}
\hline \multicolumn{3}{|c|}{$69-47-18 c c$} \\
\hline Ogallala formation: & & \\
\hline Clay............... & 70 & 70 \\
\hline 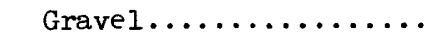 & 25 & 95 \\
\hline 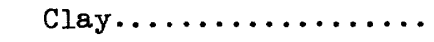 & 55 & 150 \\
\hline 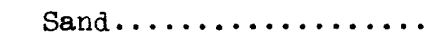 & 12 & 162 \\
\hline (No sample)........... & 8 & 170 \\
\hline Clay, yellow......... & 10 & 180 \\
\hline Pierre shale: & & \\
\hline Shale, blue.......... & 10 & 190 \\
\hline
\end{tabular}

\begin{tabular}{c|c|c}
\multicolumn{3}{c}{ C9-47-18d } \\
\hline $\begin{array}{l}\text { Ogallala formation: } \\
\text { Sand................... }\end{array}$ & 60 & 60
\end{tabular}


Table 2.--Logs of test holes, seismograph shot holes, and wells--Continued

Kit Carson County--Continued

\begin{tabular}{l|c|c}
\hline & $\begin{array}{c}\text { Thickness } \\
\text { (feet) }\end{array}$ & $\begin{array}{c}\text { Depth } \\
\text { (feet) }\end{array}$ \\
\hline
\end{tabular}

c9-47-18d--Continued

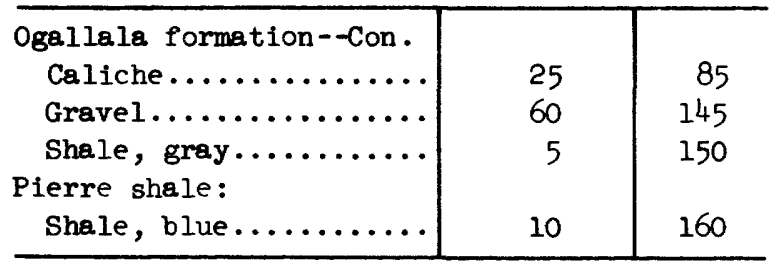

\section{c9-47-19cc}

\begin{tabular}{|c|c|c|}
\hline Cgallala formation: & & \\
\hline Clay.................. & 60 & 60 \\
\hline Gravel.............. & 20 & 80 \\
\hline 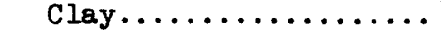 & 30 & 110 \\
\hline Clay, yellow............ & 5 & 115 \\
\hline Pierre shale: & & \\
\hline Shale, blue............ & 5 & 120 \\
\hline
\end{tabular}

C9-47-24aa. Altitude, 4,441 feet

\begin{tabular}{|c|c|c|}
\hline 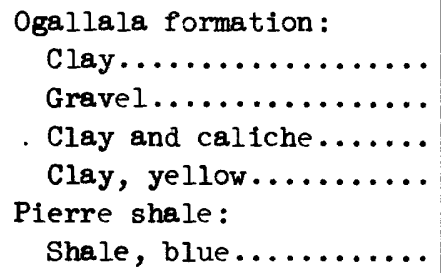 & $\begin{array}{r}30 \\
30 \\
175 \\
10 \\
\\
5\end{array}$ & $\begin{array}{r}30 \\
60 \\
235 \\
245 \\
\\
250\end{array}$ \\
\hline \multicolumn{3}{|c|}{$c 9-47-30 z$} \\
\hline 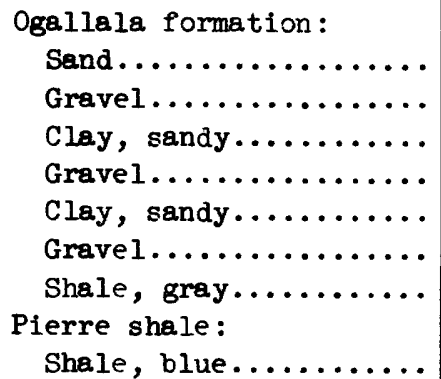 & $\begin{array}{l}10 \\
40 \\
10 \\
20 \\
10 \\
10 \\
10 \\
10\end{array}$ & $\begin{array}{r}10 \\
50 \\
60 \\
80 \\
90 \\
100 \\
110\end{array}$ \\
\hline
\end{tabular}

C9-47-31a

\begin{tabular}{|c|c|c|}
\hline \multirow{2}{*}{\multicolumn{3}{|c|}{ Ogallala formation: }} \\
\hline & & \\
\hline 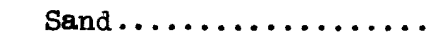 & 10 & 10 \\
\hline Gravel.............. & 10 & 20 \\
\hline Caliche.............. & 30 & 50 \\
\hline 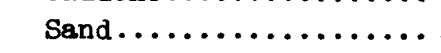 & 40 & 90 \\
\hline Clay, gray... & 5 & 95 \\
\hline
\end{tabular}

Table 2.--Logs of test holes, seismograph shot holes, and wells--Continued

Kit Carson County--Continued

\begin{tabular}{l|c|c}
\hline & $\begin{array}{c}\text { Thickness } \\
\text { (feet) }\end{array}$ & $\begin{array}{c}\text { Depth } \\
\text { (feet) }\end{array}$ \\
\hline
\end{tabular}

C9-47-31a--Continued

\begin{tabular}{|c|c|c|}
\hline $\begin{array}{l}\text { Ogallala formation--Con. } \\
\text { Shale, gray........... } \\
\text { Pierre shale: } \\
\text { Shale, blue........... }\end{array}$ & 10 & 105 \\
\hline
\end{tabular}

$\mathrm{c} 9-47-31 \mathrm{bb}$

\begin{tabular}{|c|c|c|}
\hline Ogallala formation: & & \\
\hline 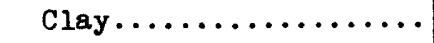 & 120 & 120 \\
\hline Gravel............. & 10 & 130 \\
\hline Clay, yellow.......... & 20 & 150 \\
\hline Pierre shale: & & \\
\hline Shale, blue.......... & 8 & 158 \\
\hline$c 9-48-3 k$ & & \\
\hline Ogallala formation: & & \\
\hline 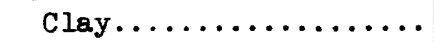 & 40 & 40 \\
\hline 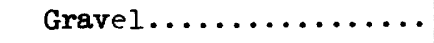 & 50 & 90 \\
\hline Clay .............. & 28 & 118 \\
\hline Clay, yellow.......... & 16 & 134 \\
\hline Pierre shale: & & \\
\hline Shale, blue.......... & 6 & 140 \\
\hline
\end{tabular}

c9-48-4cc

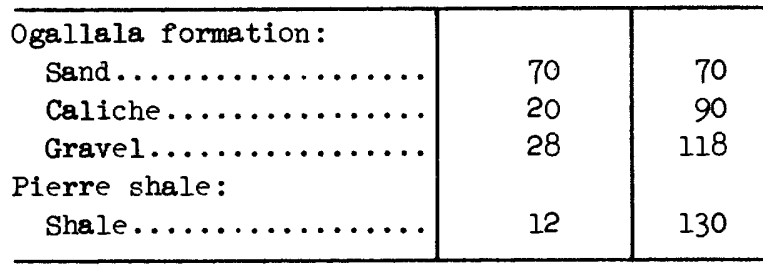

\begin{tabular}{c|c|c}
\hline \multicolumn{3}{c|}{ C9-48-5a } \\
\hline Ogallala formation: & 150 & 150 \\
Sand and gravel........ & 150 & 160 \\
Pierre shale: & 10 & 160 \\
Shale, blue..........
\end{tabular}

$c 9-48-8 c c$

\begin{tabular}{|c|c|c|}
\hline \multicolumn{3}{|l|}{ Ogallala formation: } \\
\hline Gravel............. & 89 & 129 \\
\hline Clay, yellow.......... & 21 & 150 \\
\hline Pierre shale: & & \\
\hline Shale, blue.......... & 10 & 160 \\
\hline
\end{tabular}


Table 2.--Logs of test holes, seismograph shot holes, and wells--Continued

Kit Carson County--Continued

\begin{tabular}{|c|c|c|}
\hline & $\begin{array}{c}\text { Thickness } \\
\text { (feet) }\end{array}$ & $\begin{array}{l}\text { Depth } \\
\text { (feet) }\end{array}$ \\
\hline \multicolumn{3}{|c|}{ c9-48-17aa } \\
\hline Ogallala formation: & & \\
\hline Sand $\ldots \ldots \ldots \ldots \ldots$ & 60 & 60 \\
\hline 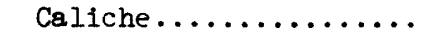 & 20 & 80 \\
\hline Gravel............. & 20 & 100 \\
\hline Pierre shale: & & \\
\hline Shale.............. & 10 & 110 \\
\hline
\end{tabular}

c9-48-17dd

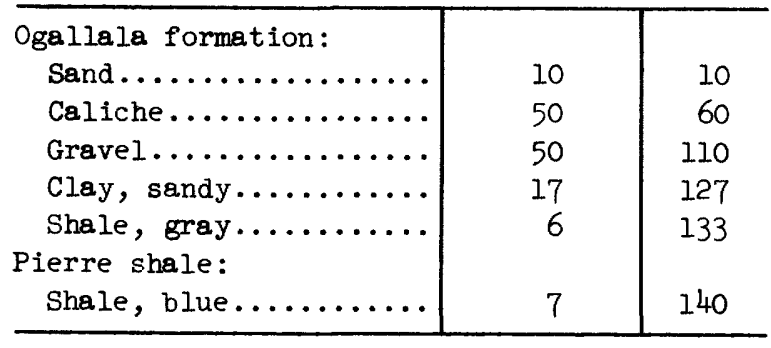

$\mathrm{C} 9-48-20 \mathrm{~b}$

\begin{tabular}{|c|c|c|}
\hline \multicolumn{3}{|l|}{ Ogallala formation: } \\
\hline 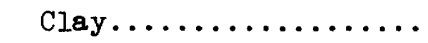 & 20 & 20 \\
\hline Gravel............. & 40 & 60 \\
\hline Clay, yellow......... & 48 & 108 \\
\hline Pierre shale: & & \\
\hline Shale, blue.......... & 12 & 120 \\
\hline
\end{tabular}

\section{$\mathrm{c} 9-48-31 \mathrm{bb}$}

\begin{tabular}{|c|c|c|}
\hline Ogallala formation: & & \\
\hline Sand and gravel....... & 150 & 150 \\
\hline Clay, sandy.......... & 20 & 170 \\
\hline $\begin{array}{l}\text { Shale, gray } \ldots \ldots \ldots \ldots \ldots \\
\text { Pierre shale: }\end{array}$ & 5 & 175 \\
\hline Shale, blue.......... & 5 & 180 \\
\hline
\end{tabular}

\section{c9-48-31d}

\begin{tabular}{c|c|c}
\hline Ogallala formation. & & \\
Clay.............. & 15 & 15 \\
Sand and gravel, with & & \\
small breaks of clay.. & 150 & 165 \\
Clay, yellow......... & 10 & 175 \\
Pierre shale $\ldots \ldots \ldots$ & 5 & 180 \\
Shale, blue......... & 5 \\
\hline
\end{tabular}

$\mathrm{c} 9-48-32 \mathrm{bb}$

\begin{tabular}{l|l|l}
\hline Ogallala formation: & \\
Clay and caliche...... & 65 & 65
\end{tabular}

Table 2.--Logs of test holes, seismograph shot holes, and wells--Continued

Kit Carson County--Continued

\begin{tabular}{|c|c|c|}
\hline & $\begin{array}{c}\text { Thickness } \\
\text { (feet) }\end{array}$ & $\begin{array}{l}\text { Depth } \\
\text { (feet) }\end{array}$ \\
\hline \multicolumn{3}{|c|}{$c 9-48-32 b b--c o n t i n u e d$} \\
\hline $\begin{array}{l}\text { Ogallala formation--Con. } \\
\text { Gravel............ } \\
\text { Clay, gray......... } \\
\text { Clay, yellow........ } \\
\text { Pierre shale: } \\
\text { Shale, blue......... }\end{array}$ & $\begin{array}{l}45 \\
35 \\
17\end{array}$ & $\begin{array}{l}110 \\
145 \\
162 \\
170\end{array}$ \\
\hline
\end{tabular}

\section{$c 9-48-34 c$}

\begin{tabular}{l|r|r}
\hline Ogallala formation: & & \\
Sand and gravel....... & 125 & 125 \\
Clay, yellow......... & 9 & 134 \\
Pierre shale: & & \\
Shale, blue.......... & 6 & 140 \\
\hline
\end{tabular}

\begin{tabular}{|c|c|c|}
\hline \multicolumn{3}{|l|}{$c 9-49-1 a$} \\
\hline Ogallala formation: & & \\
\hline 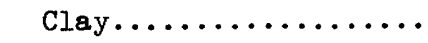 & 50 & 50 \\
\hline Sand and gravel....... & 70 & 120 \\
\hline Clay, yellow......... & 32 & 152 \\
\hline Pierre shale: & & \\
\hline Shale, blue.......... & 8 & 160 \\
\hline
\end{tabular}

\begin{tabular}{|c|c|c|}
\hline \multicolumn{3}{|c|}{ C9-49-1dd } \\
\hline Ogallala formation: & & \\
\hline 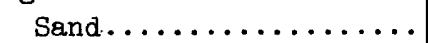 & 90 & 90 \\
\hline Gravel............. & 10 & 100 \\
\hline Sand $\ldots \ldots \ldots \ldots \ldots$ & 30 & 130 \\
\hline Clay, yellow........... & 12 & 142 \\
\hline Pierre shale: & & \\
\hline Shale, blue.......... & 8 & 150 \\
\hline
\end{tabular}

\section{C9-49-13a8}

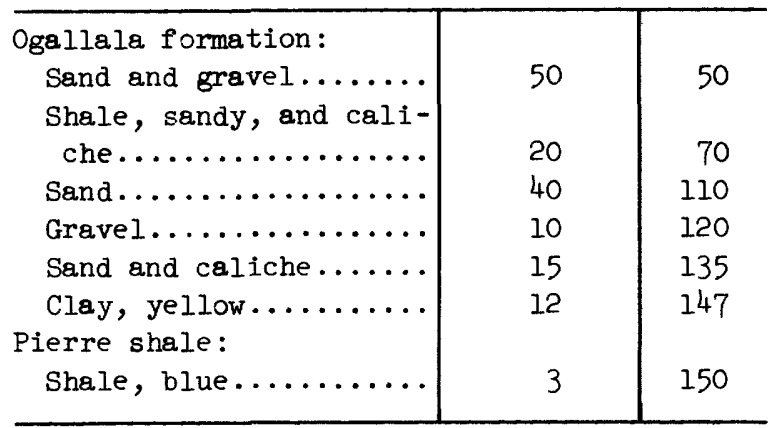


Table 2.--Logs of test holes, seismograph shot holes, and wells--Continued

Kit Carson County--Continued

\begin{tabular}{|c|c|c|}
\hline . & $\begin{array}{c}\text { Thickness } \\
\text { (feet) }\end{array}$ & $\begin{array}{l}\text { Depth } \\
\text { (f eet) }\end{array}$ \\
\hline \multicolumn{3}{|c|}{ c9-49-2laa } \\
\hline \multicolumn{3}{|l|}{ Ogallala formation: } \\
\hline 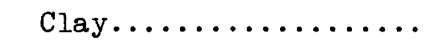 & 20 & 20 \\
\hline Gravel............. & 25 & 45 \\
\hline Sand and sandy clay.... & 65 & 110 \\
\hline Caliche............. & 20 & 130 \\
\hline 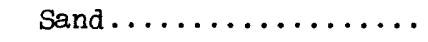 & 25 & 155 \\
\hline Clay, yellow......... & 20 & 175 \\
\hline \multicolumn{3}{|l|}{ Pierre shale: } \\
\hline Shale, blue... & 5 & 180 \\
\hline
\end{tabular}

\section{C9-49-24aa}

\begin{tabular}{|c|c|c|}
\hline \multirow{3}{*}{$\begin{array}{l}\text { Ogallala formation: } \\
\text { Shale, sandy } \ldots \ldots \ldots \ldots \\
\text { Gravel } \ldots \ldots \ldots \ldots \ldots\end{array}$} & & \\
\hline & 50 & 50 \\
\hline & 15 & 65 \\
\hline 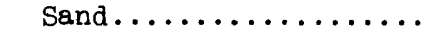 & 25 & 90 \\
\hline Shale, sandy..$\ldots \ldots \ldots$ & 20 & 110 \\
\hline $\operatorname{Sand} \ldots \ldots \ldots \ldots \ldots \ldots$ & 15 & 125 \\
\hline Clay, yellow........... & 10 & 135 \\
\hline Pierre shale: & & \\
\hline Shale, blue........... & 5 & 140 \\
\hline
\end{tabular}

c9-49-24dd

\begin{tabular}{|c|c|c|}
\hline \multicolumn{3}{|l|}{ Ogallala formation: } \\
\hline 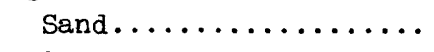 & 60 & 60 \\
\hline Gravel.............. & 10 & 70 \\
\hline Shale, sandy........... & 40 & 110 \\
\hline Clay, yellow........... & 10 & 120 \\
\hline Pierre shale: & & \\
\hline Shale, blue............ & 10 & 130 \\
\hline
\end{tabular}

\begin{tabular}{|c|c|c|}
\hline \multicolumn{3}{|c|}{ C9-50-1aa } \\
\hline Ogallala formation: & & \\
\hline 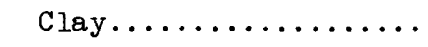 & 15 & 15 \\
\hline Gravel............... & 23 & 38 \\
\hline Clay and caliche....... & 19 & 57 \\
\hline Clay, yellow........... & 28 & 85 \\
\hline Pierre shale: & & \\
\hline Shale, blue........... & 15 & 100 \\
\hline
\end{tabular}

C9-50-28aa

\begin{tabular}{|c|c|c|}
\hline $\begin{array}{l}\text { Ogallala formation: } \\
\text { Clay } \ldots \ldots \ldots \ldots \ldots \ldots \ldots \\
\text { Gravel } \ldots \ldots \ldots \ldots \ldots \ldots\end{array}$ & $\begin{array}{l}30 \\
30\end{array}$ & $\begin{array}{l}30 \\
60\end{array}$ \\
\hline $\begin{array}{c}\text { Clay and caliche, with } \\
\text { breaks of gravel...... }\end{array}$ & 88 & 148 \\
\hline
\end{tabular}

Table 2.--Logs of test holes, seismograph shot holes, and wells--Continued

Kit Carson County--Continued

\begin{tabular}{l|c|c}
\hline & $\begin{array}{c}\text { Thickness } \\
\text { (feet) }\end{array}$ & $\begin{array}{c}\text { Depth } \\
\text { (feet) }\end{array}$ \\
\hline
\end{tabular}

C9-50-28aa--Continued

\begin{tabular}{l|c|c}
\hline Ogallala formation--Con. & & \\
Clay, yellow........... & 16 & 164 \\
Pierre shale: & & \\
Shale, blue........... & 6 & 170 \\
\hline
\end{tabular}

\section{C9-51-1aa}

\begin{tabular}{|c|c|c|}
\hline \multicolumn{3}{|l|}{ Ogallàla formation: } \\
\hline Clay.$\ldots \ldots \ldots \ldots \ldots$ & 40 & 40 \\
\hline Callche...$\ldots \ldots \ldots \ldots$ & 15 & 55 \\
\hline Sand and gravel $\ldots \ldots \ldots$ & 35 & 90 \\
\hline Clay, yellow........ & 40 & 130 \\
\hline Pierre shale: & & \\
\hline Shale, & 20 & 150 \\
\hline
\end{tabular}

C9-51-2baa. Drilled by Ben Hasz, 1948

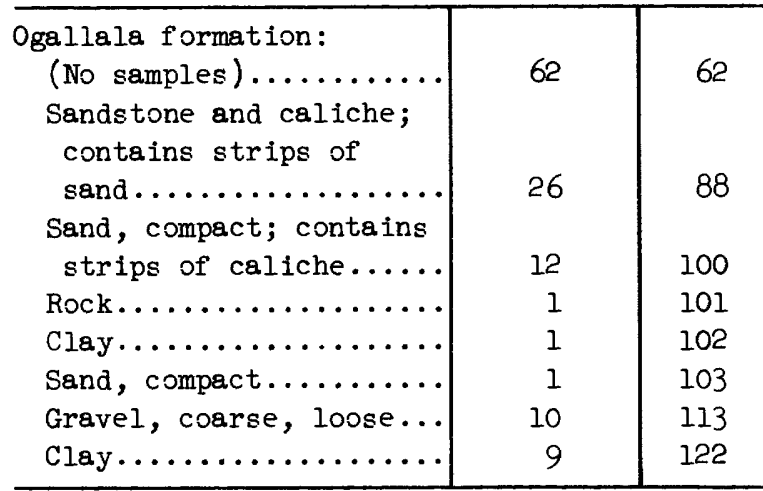

C9-51-2bab. Drilled by Ben Hasz, 1948

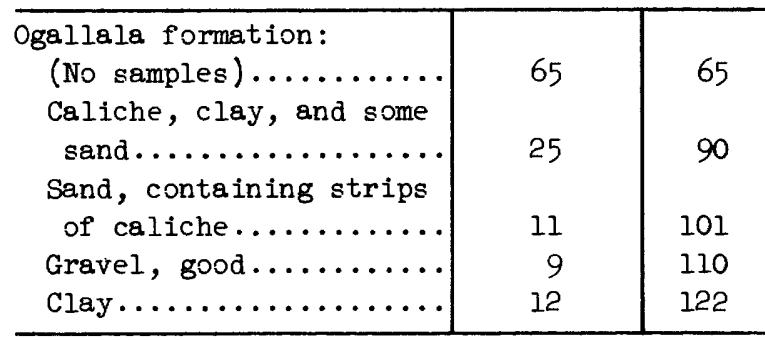

C9-5l-2bb. Drilled by Ben Hasz, 1948

\begin{tabular}{|c|c|c|}
\hline $\begin{array}{l}\text { Ogallala formation: } \\
\text { (No samples) } \ldots \ldots \ldots \ldots \ldots\end{array}$ & 62 & 62 \\
\hline Sandstone and caliche... & 13 & 75 \\
\hline $\begin{array}{c}\text { Sand, caliche, and some } \\
\text { clay } . . \ldots \ldots \ldots \ldots \ldots \ldots\end{array}$ & 5 & 80 \\
\hline 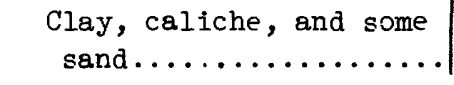 & 10 & 90 \\
\hline
\end{tabular}


Table 2.--Logs of test holes, seismograph shot holes, and wells--Continued

\section{Kit Carson County--Continued}

\begin{tabular}{l|c|c}
\hline & $\begin{array}{c}\text { Thickness } \\
\text { (feet) }\end{array}$ & $\begin{array}{c}\text { Depth } \\
\text { (feet) }\end{array}$ \\
\hline
\end{tabular}

C9-5l-2bb--Continued

\begin{tabular}{|c|c|c|}
\hline Ogallala formation--Con. & 21 & ו \\
\hline $\begin{array}{l}\text { Clay . . . } \\
\text { Pierre shale }(?):\end{array}$ & 14 & 125 \\
\hline Shale .............. & 2 & 127 \\
\hline
\end{tabular}

C9-5l-2bbb. Drilled by Ben Hasz, 1948

\begin{tabular}{|c|c|c|}
\hline 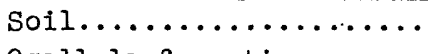 & 21 & 21 \\
\hline Ogallala formation: & 6 & ב \\
\hline 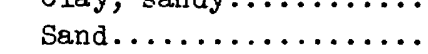 & 18 & 45 \\
\hline $\begin{array}{l}\text { Sand, containing strips } \\
\text { of caliche and clay... }\end{array}$ & 29 & 74 \\
\hline $\begin{array}{l}\text { Sand, caliche, and } \\
\text { sandy rock............ }\end{array}$ & 17 & 91 \\
\hline $\begin{array}{l}\text { Rock, sandstone, and } \\
\text { some clay............ }\end{array}$ & 12 & 103 \\
\hline Gravel, loose........ & 9 & 112 \\
\hline 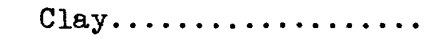 & 10 & 122 \\
\hline
\end{tabular}

C9-51-2bbc. Drilled by Ben Hasz, 1948

\begin{tabular}{|c|c|c|}
\hline 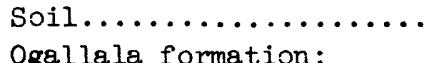 & 25 & 25 \\
\hline 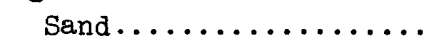 & 23 & 48 \\
\hline $\begin{array}{l}\text { Caliche and sandy clay. } \\
\text { Sand, loose, containing }\end{array}$ & 6 & 54 \\
\hline some caliche........ & 13 & 67 \\
\hline $\begin{array}{l}\text { Caliche, compact, con- } \\
\text { taining some sand..... }\end{array}$ & 15 & 82 \\
\hline $\begin{array}{l}\text { Sand, containing strips } \\
\text { of caliche............ }\end{array}$ & 11 & 93 \\
\hline $\begin{array}{l}\text { Sandstone and compact } \\
\text { sand.............. }\end{array}$ & 8 & 101 \\
\hline Gravel, loose........ & 12 & 113 \\
\hline 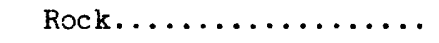 & 6 & 119 \\
\hline 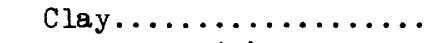 & 11 & 130 \\
\hline Pierre shale(?): & & \\
\hline 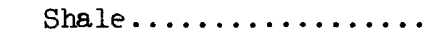 & $\cdots$ & 130 \\
\hline
\end{tabular}

C9-5l-2bcb.. Drilled by Ben Hasz, 1948

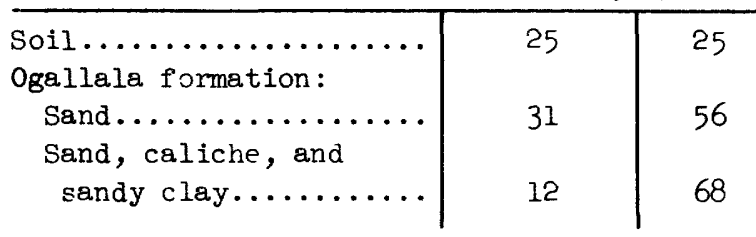

Table 2.--Logs of test holes, seismograph shot holes, and wells--Continued

Kit Carson County--Continued

\begin{tabular}{l|c|c}
\hline & $\begin{array}{c}\text { Thickness } \\
\text { (feet) }\end{array}$ & $\begin{array}{c}\text { Depth } \\
\text { (feet) }\end{array}$ \\
\hline
\end{tabular}

C9-5l-2bcb--Continued

\begin{tabular}{|c|c|c|}
\hline Ogallala formation--Con. & & \\
\hline Sand and caliche...... & 20 & 88 \\
\hline Caliche and sandstone... & 13 & 101 \\
\hline Gravel, loose........ & 10 & 111 \\
\hline Clay.............. & 11 & 122 \\
\hline
\end{tabular}

$c 9-51-6 b b$

\begin{tabular}{|c|c|c|}
\hline Ogallala formation: & & \\
\hline Clay and caliche...... & 80 & 80 \\
\hline Gravel............ & 20 & 100 \\
\hline Caliche............ & 15 & 115 \\
\hline Clay, gray........... & 10 & 125 \\
\hline Pierre shale: & & \\
\hline Shale, blue.......... & 5 & 130 \\
\hline
\end{tabular}

\begin{tabular}{|c|c|c|}
\hline \multicolumn{3}{|c|}{ C9-5l-2las. } \\
\hline 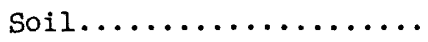 & 5 & 5 \\
\hline Ogallala formation: & & \\
\hline 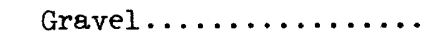 & 7 & 12 \\
\hline Pierre shale: & & \\
\hline Shale, blue.......... & 108 & 120 \\
\hline
\end{tabular}

c9-5l-3lcc

\begin{tabular}{|c|c|c|}
\hline 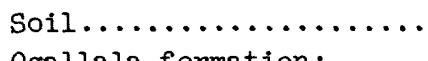 & 3 & 3 \\
\hline Ogallala formation: & & \\
\hline Gravel............. & 6 & 9 \\
\hline Clay, gray and yellow.. & 61 & 70 \\
\hline Pierre shale: & & \\
\hline Shale, blue.......... & 10 & 80 \\
\hline
\end{tabular}

\section{Clo-42-3lddl}

\begin{tabular}{|c|c|c|}
\hline Ogallala formation: & & \\
\hline Shale, sandy......... & 15 & 15 \\
\hline Gravel and caliche.... & 25 & 40 \\
\hline 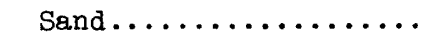 & 15 & 55 \\
\hline Shale, sandy......... & 25 & 80 \\
\hline Caliche............ & 10 & 90 \\
\hline Sand................ & 75 & 165 \\
\hline Clay, yellow......... & 20 & 185 \\
\hline Shale, gray.......... & 5 & 190 \\
\hline Pierre shale: & & \\
\hline Shale, blue.......... & 10 & 200 \\
\hline
\end{tabular}


Table 2.--Logs of test holes, seismograph shot holes, and wells--Continued

Kit Carson County--Continued

\begin{tabular}{|c|c|c|}
\hline & $\begin{array}{c}\text { Thickness } \\
\text { (feet) }\end{array}$ & $\begin{array}{l}\text { Depth } \\
\text { (feet) }\end{array}$ \\
\hline \multicolumn{3}{|c|}{ c10-42-31dd2 } \\
\hline $\begin{array}{l}\text { Ogallala formation: } \\
\text { Clay and caliche...... } \\
\text { Clay, yellow......... } \\
\text { Pierre shale: } \\
\text { Shale, blue.......... }\end{array}$ & $\begin{array}{r}180 \\
10 \\
10\end{array}$ & $\begin{array}{l}180 \\
190 \\
200\end{array}$ \\
\hline C10-42-35cc. Altit & le, 4,002 $\mathrm{f}$ & \\
\hline
\end{tabular}

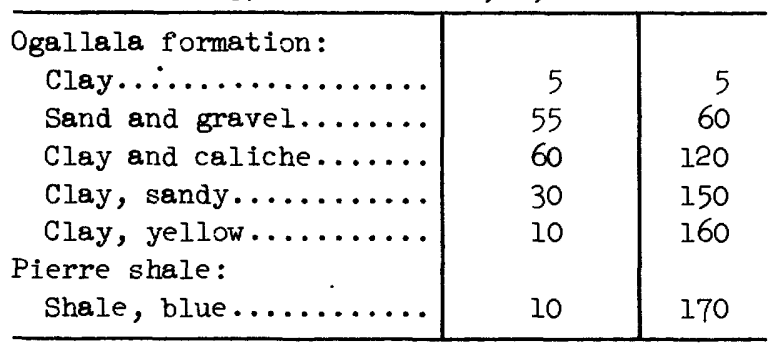

C10-43-25bb. Drilled by K. G. Wilcox, 1948

\begin{tabular}{|c|c|c|}
\hline $\begin{array}{l}\text { Soil ................ } \\
\text { Ogallala formation: }\end{array}$ & 3 & 3 \\
\hline Clay, yellow.......... & 32 & 35 \\
\hline Caliche............. & 5 & 40 \\
\hline Gravel............. & 16 & 56 \\
\hline Caliche............. & 19 & 75 \\
\hline Gravel............. & 7 & 82 \\
\hline Conglomerate.......... & 3 & 85 \\
\hline Clay, sandy, and grav- & & \\
\hline el $\ldots \ldots \ldots \ldots \ldots \ldots$ & 6 & 91 \\
\hline 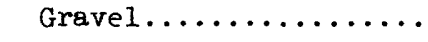 & 6 & 97 \\
\hline Sandstone............ & 5 & 102 \\
\hline Sand and gravel....... & 8 & 110 \\
\hline Caliche............. & 4 & 114 \\
\hline Gravel............. & 3 & 117 \\
\hline Conglomerate......... & 8 & 125 \\
\hline Sandstone ............ & 4 & 129 \\
\hline Gravel............. & 9 & 138 \\
\hline Clay................ & 2 & 140 \\
\hline Conglomerate......... & 5 & 145 \\
\hline Gravel.............. & 1 & 146 \\
\hline Clay, white......... & 2 & 148 \\
\hline Conglomera te .......... & 6 & 154 \\
\hline Gravel............. & 2 & 156 \\
\hline Rock, hard.......... & 4 & 160 \\
\hline Gravel............. & 3 & 163 \\
\hline 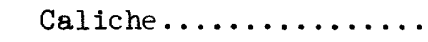 & 5 & 168 \\
\hline 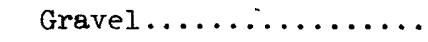 & 25 & 193 \\
\hline Clay................ & 7 & 200 \\
\hline
\end{tabular}

Table 2.--Logs of test holes, seismograph shot holes, and wells--Continued

Kit Carson County--Continued

\begin{tabular}{c|c|c}
\hline & $\begin{array}{c}\text { Thickness } \\
\text { (feet) }\end{array}$ & $\begin{array}{c}\text { Depth } \\
\text { (feet) }\end{array}$ \\
\hline
\end{tabular}

C10-43-27cc. Drilled by K. G. Wilcox, 1948

\begin{tabular}{|c|c|c|}
\hline 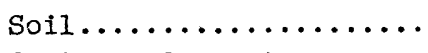 & 4 & 4 \\
\hline \multicolumn{3}{|l|}{ Ogallala formation: } \\
\hline Clay, yellow.......... & 38 & 42 \\
\hline Clay, sandy.......... & 6 & 48 \\
\hline Conglomerate......... & 1 & 49 \\
\hline Caliche............ & 2 & 51 \\
\hline 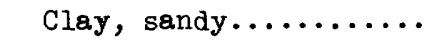 & 3 & 54 \\
\hline Caliche, hard......... & 20 & 74 \\
\hline Gravel............ & 4 & 78 \\
\hline Conglomerate......... & 6 & 84 \\
\hline Gravel............. & 5 & 89 \\
\hline Caliche............. & 23 & 112 \\
\hline 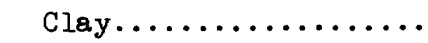 & 3 & 115 \\
\hline Gravel............. & 14 & 129 \\
\hline Caliche, hard........ & 3 & 132 \\
\hline Conglomerate......... & 5 & 137 \\
\hline Caliche............. & 5 & 142 \\
\hline Gravel............. & 4 & 146 \\
\hline Clay.............. & 2 & 148 \\
\hline Caliche............. & 7 & 155 \\
\hline 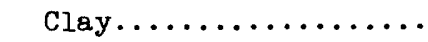 & 3 & 158 \\
\hline 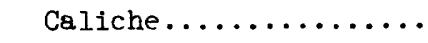 & 1 & 159 \\
\hline Clay............... & 1 & 160 \\
\hline Caliche............. & 5 & 165 \\
\hline Gravel............. & 5 & 170 \\
\hline Caliche............. & 2 & 172 \\
\hline Gravel............ & 8 & 180 \\
\hline 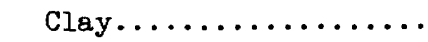 & 2 & 182 \\
\hline Gravel............. & 22 & 204 \\
\hline Clay, yellow......... & 16 & 220 \\
\hline Gravel............. & 5 & 225 \\
\hline 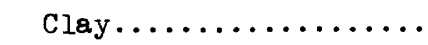 & 4 & 229 \\
\hline Gravel............. & 12 & 241 \\
\hline Clay, yellow.......... & 5 & 246 \\
\hline 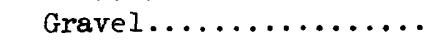 & 15 & 261 \\
\hline Pierre shale: & & \\
\hline Shale, blue.......... & 1 & 262 \\
\hline
\end{tabular}

C10-43-35bb. Drilled by K. G. Wilcox, 1948

\begin{tabular}{|c|c|c|}
\hline 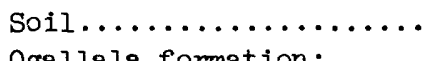 & 5 & 5 \\
\hline Ogallala formation: & 42 & 47 \\
\hline 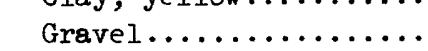 & 21 & 68 \\
\hline Caliche............. & 3 & 71 \\
\hline Clay, sandy.......... & 9 & 80 \\
\hline Caliche.............. & 22 & 102 \\
\hline Clay.............. & 3 & 105 \\
\hline
\end{tabular}


Table 2.--Logs of test holes, seismograph shot holes, and wells--Continued

Kit Carson County--Continued

\begin{tabular}{l|c|c}
\hline & $\begin{array}{c}\text { Thickness } \\
\text { (feet) }\end{array}$ & $\begin{array}{c}\text { Depth } \\
\text { (feet) }\end{array}$ \\
\hline
\end{tabular}

C10-43-35bb--Continued

\begin{tabular}{|c|c|c|}
\hline \multirow{2}{*}{\multicolumn{3}{|c|}{ Ogallala formation--Con. }} \\
\hline & 6 & 111 \\
\hline 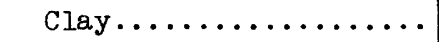 & 4 & 115 \\
\hline Conglomerate......... & 2 & 117 \\
\hline Clay, sandy $\ldots \ldots \ldots \ldots$ & 2 & 119 \\
\hline Conglomerate......... & 1 & 120 \\
\hline Clay, sandy .......... & 2 & 122 \\
\hline Sandstone............ & 1 & 123 \\
\hline Grave1.............. & 5 & 128 \\
\hline Caliche............. & 6 & 134 \\
\hline Clay, sandy.......... & 3 & 137 \\
\hline Gravel, loose........ & 28 & 165 \\
\hline 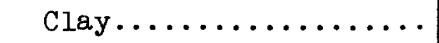 & 1 & 166 \\
\hline Gravel.............. & 12 & 178 \\
\hline 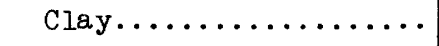 & 2 & 180 \\
\hline Clay, sandy......... & 34 & 214 \\
\hline Sand, fine, loose..... & 16 & 230 \\
\hline 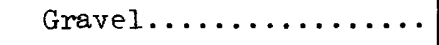 & 3 & 233 \\
\hline Caliche............. & 1 & 234 \\
\hline Sand, coarse......... & 12 & 246 \\
\hline \multicolumn{3}{|l|}{ Pierre shale: } \\
\hline Shale, blue.......... & 1 & 247 \\
\hline \multicolumn{3}{|c|}{ C10-44-13dd } \\
\hline \multicolumn{3}{|l|}{ Ogallala formation: } \\
\hline Clay and caliche...... & 110 & 110 \\
\hline (No sample $\lambda \ldots \ldots \ldots \ldots$. & 160 & 270 \\
\hline Clay, gray........... & 20 & 290 \\
\hline \multicolumn{3}{|l|}{ Pierre shale: } \\
\hline Shale, blue.......... & $\cdots$ & 290 \\
\hline
\end{tabular}

c10-44-16dd. Altitude, 4,248 feet

\begin{tabular}{|c|c|c|}
\hline \multicolumn{3}{|l|}{ Ogallala formation: } \\
\hline 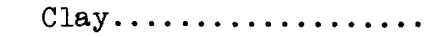 & 30 & 30 \\
\hline Grave1............ & 50 & 80 \\
\hline Clay and caliche...... & 110 & 190 \\
\hline Gravel............ & 80 & 270 \\
\hline Clay, gray........... & 10 & 280 \\
\hline Pierre shale: & & \\
\hline Shale, blue.......... & 10 & 290 \\
\hline
\end{tabular}

C10-44-31c. Altitude, 4,306 feet

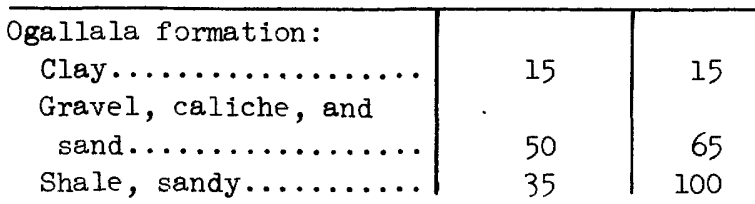

Table 2.--Logs of test holes, seismograph shot holes, and wells--Continued

Kit Carson County--Continued

\begin{tabular}{l|c|c}
\hline & $\begin{array}{c}\text { Thickness } \\
\text { (feet) }\end{array}$ & $\begin{array}{c}\text { Depth } \\
\text { (feet) }\end{array}$ \\
\hline
\end{tabular}

C10-44-31c--Continued

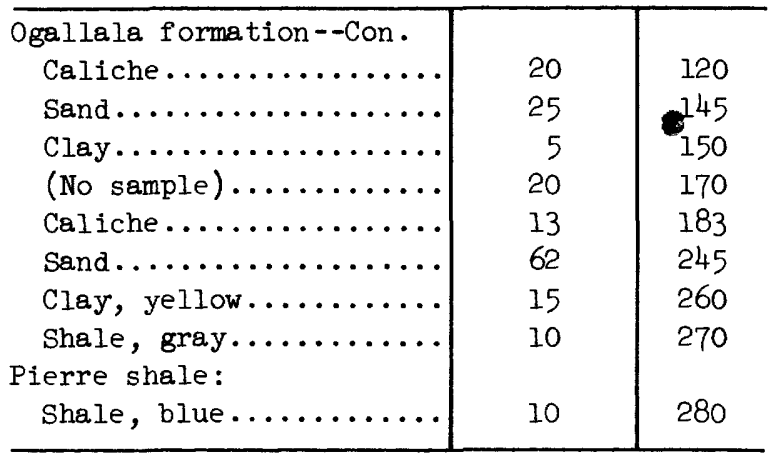

\section{$\mathrm{C} 10-44-36 \mathrm{~d}$}

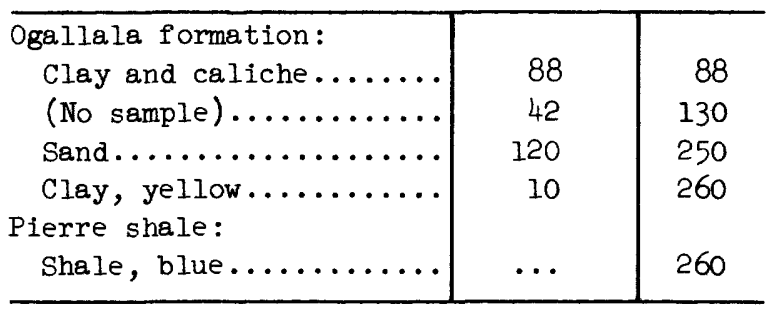

C10-45-2aa. Altitude, 4,243 feet

\begin{tabular}{|c|c|c|}
\hline Ogallala formation: & & \\
\hline 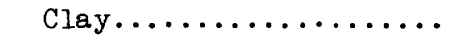 & 28 & 28 \\
\hline Gravel and boulders..... & 92 & 120 \\
\hline Clay and caliche....... & 80 & 200 \\
\hline Sand and gravel........ & 35 & 235 \\
\hline Clay, yellow........... & 10 & 245 \\
\hline Pierre shale: & & \\
\hline Shale, blue........... & 5 & 250 \\
\hline
\end{tabular}

C10-45-16dd. Altitude, 4,296 feet

\begin{tabular}{|c|c|c|}
\hline Ogallala formation: & & \\
\hline 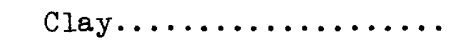 & 20 & 20 \\
\hline Gravel.............. & 40 & 60 \\
\hline Clay and caliche....... & 110 & 170 \\
\hline Sand and gravel........ & 70 & 240 \\
\hline Clay, yellow........... & 10 & 250 \\
\hline Pierre shale: & & \\
\hline Shale, blue.......... & 10 & 260 \\
\hline
\end{tabular}

C10-45-19b. Altitude, 4,397 feet

\begin{tabular}{|c|c|c|}
\hline 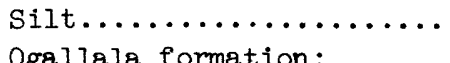 & 15 & 15 \\
\hline 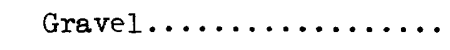 & 15 & 30 \\
\hline
\end{tabular}


Table 2.--Logs of test holes, seismograph shot holes, and wells--Continued

Kit Carson County--Continued

\begin{tabular}{|c|c|c|}
\hline & $\begin{array}{c}\text { Thickmess } \\
\text { (feet) }\end{array}$ & $\begin{array}{l}\text { Depth } \\
\text { (feet) }\end{array}$ \\
\hline \multicolumn{3}{|c|}{ C10-45-19b--Continued } \\
\hline 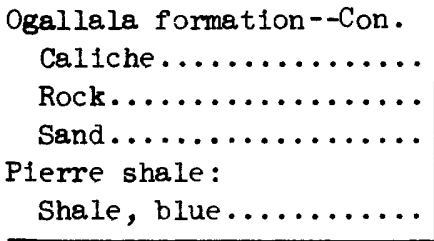 & $\begin{array}{r}120 \\
10 \\
120 \\
10\end{array}$ & $\begin{array}{l}150 \\
160 \\
280 \\
290\end{array}$ \\
\hline C10-45-33c. A1t1t & le, $4,373 \mathrm{f}$ & \\
\hline 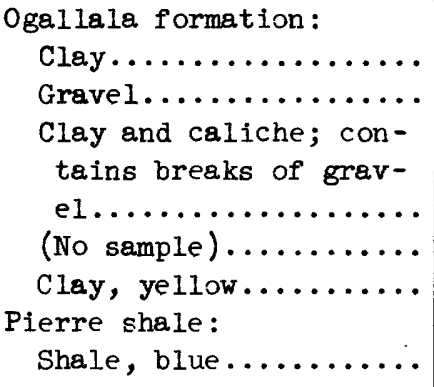 & $\begin{array}{r}105 \\
95 \\
30 \\
10\end{array}$ & $\begin{array}{r}23 \\
50 \\
\\
155 \\
250 \\
280 \\
290\end{array}$ \\
\hline
\end{tabular}

C10-46-16da. Altitude, 4,442 feet

\begin{tabular}{|c|c|c|}
\hline Silt and clay.......... & 55 & 55 \\
\hline \multicolumn{3}{|l|}{ Ogallala formation: } \\
\hline & 45 & 100 \\
\hline 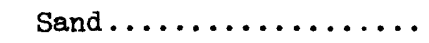 & 80 & 180 \\
\hline Clay, red............ & 10 & 190 \\
\hline 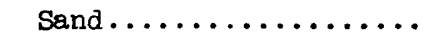 & 55 & 245 \\
\hline Shale, gray.......... & 17 & 262 \\
\hline \multicolumn{3}{|l|}{ Pierre shale: } \\
\hline Shale, blue.......... & 8 & 270 \\
\hline
\end{tabular}

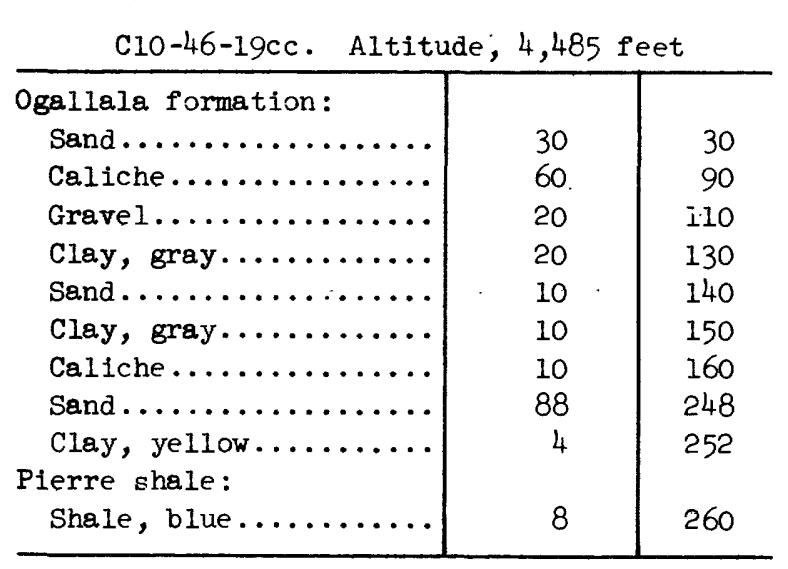

Table 2.--Logs of test holes, seismograph shot holes, and wells--Continued

\section{Kit Carson County--Continued}

\begin{tabular}{l|c|c}
\hline & $\begin{array}{c}\text { Thickness } \\
\text { (feet) }\end{array}$ & $\begin{array}{c}\text { Depth } \\
\text { (feet) }\end{array}$ \\
\hline
\end{tabular}

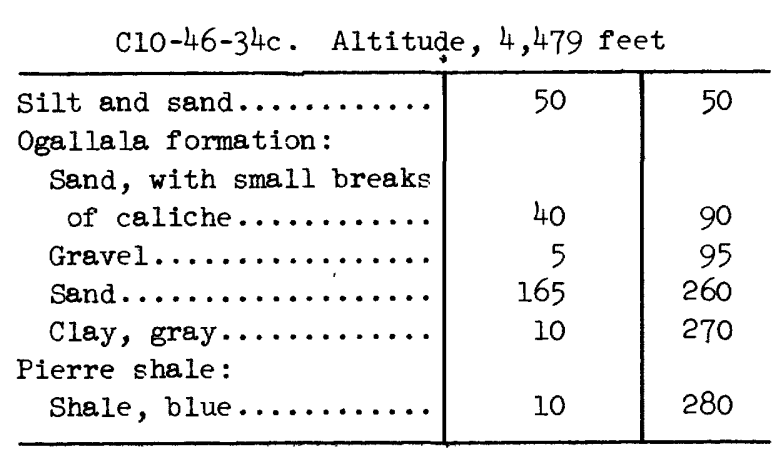

\begin{tabular}{|c|c|c|}
\hline \multicolumn{3}{|c|}{$C 10-47-18 a$} \\
\hline Soil.............. & 8 & 8 \\
\hline Ogallala formation: & & \\
\hline Clay and conglomerate.. & 180 & 188 \\
\hline Sand (water).......... & 12 & 200 \\
\hline Pierre shale: & & \\
\hline Shale............. & . & 200 \\
\hline
\end{tabular}

\begin{tabular}{|c|c|c|}
\hline \multicolumn{3}{|c|}{$C 10-48-2 b b$} \\
\hline Ogallala formation: & & \\
\hline 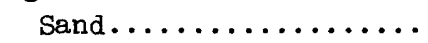 & 20 & 20 \\
\hline Shale, sandy......... & 50 & 70 \\
\hline Grave1 and sand....... & 50 & 120 \\
\hline Shale, sandy......... & 10 & 130 \\
\hline 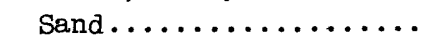 & 15 . & 145. \\
\hline Clay, yellow.......... & 7 & 152 \\
\hline Pierre shale: & & \\
\hline Shale, blue.......... & 8 & 160 \\
\hline
\end{tabular}

\begin{tabular}{|c|c|c|}
\hline \multicolumn{3}{|c|}{$c 10-48-4 c c$} \\
\hline $\begin{array}{l}\text { Ogallala formation: } \\
\text { Sand and gravel....... } \\
\text { Clay, yellow......... } \\
\text { Pierre shale: } \\
\text { Shale, blue........... }\end{array}$ & $\begin{array}{r}170 \\
7 \\
3\end{array}$ & $\begin{array}{l}170 \\
177 \\
180\end{array}$ \\
\hline \multicolumn{3}{|c|}{ C10-48-5aa } \\
\hline 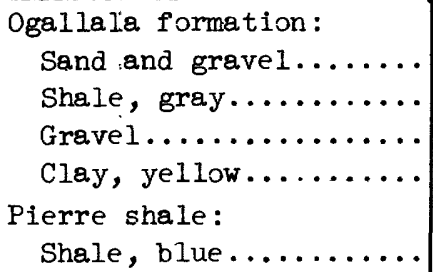 & $\begin{array}{l}90 \\
20 \\
20 \\
15 \\
5\end{array}$ & $\begin{array}{r}90 \\
110 \\
130 \\
145 \\
150\end{array}$ \\
\hline
\end{tabular}


Table 2.--Logs of test holes, seismograph shot holes, and wells--Continued

Kit Carson County--Continued

\begin{tabular}{l|c|c}
\hline & $\begin{array}{c}\text { Thickness } \\
\text { (feet) }\end{array}$ & $\begin{array}{c}\text { Depth } \\
\text { (feet) }\end{array}$ \\
\hline
\end{tabular}

$\mathrm{c} 10-48-9 \mathrm{cc}$

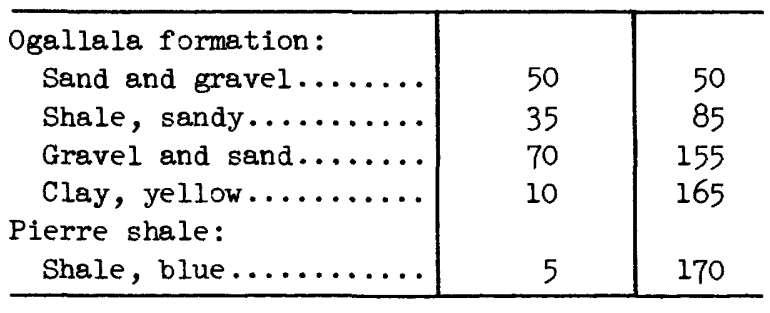

C10-48-10d

\begin{tabular}{|c|c|c|}
\hline $\begin{array}{l}\text { Ogallala formation: } \\
\text { Sand and gravel....... } \\
\text { Shale, gray.......... } \\
\text { Clay, yellow......... } \\
\text { Pierre shale: } \\
\text { Shale, blue.......... }\end{array}$ & $\begin{array}{r}120 \\
5 \\
5 \\
20\end{array}$ & $\begin{array}{l}120 \\
125 \\
130 \\
150\end{array}$ \\
\hline \multicolumn{3}{|c|}{$\mathrm{C} 10-48-11 \mathrm{bb}$} \\
\hline $\begin{array}{l}\text { Ogallala formation: } \\
\text { Sand and gravel....... } \\
\text { Clay, yellow......... } \\
\text { Pierre shale: } \\
\text { Shale, blue.......... }\end{array}$ & $\begin{array}{r}145 \\
5 \\
10\end{array}$ & $\begin{array}{l}145 \\
150 \\
160\end{array}$ \\
\hline
\end{tabular}

\section{$\mathrm{C} 10-48-17 \mathrm{~d}$}

\begin{tabular}{|c|c|c|}
\hline 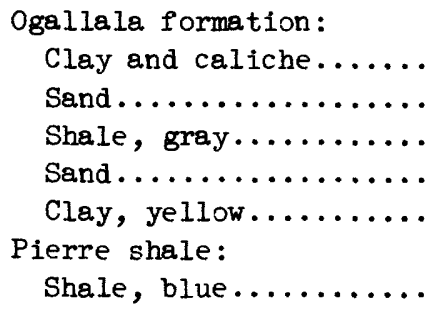 & $\begin{array}{r}50 \\
35 \\
5 \\
65 \\
5 \\
20\end{array}$ & $\begin{array}{r}50 \\
85 \\
90 \\
155 \\
160 \\
180\end{array}$ \\
\hline \multicolumn{3}{|c|}{ C10-48-20d } \\
\hline $\begin{array}{l}\text { Ogallala formation: } \\
\text { Sand and sandy shale... } \\
\text { Gravel............ } \\
\text { Sand and gravel....... } \\
\text { Clay, yellow........ } \\
\text { Pierre shale: } \\
\text { Shale, blue......... }\end{array}$ & $\begin{array}{r}50 \\
15 \\
70 \\
5\end{array}$ & $\begin{array}{r}50 \\
65 \\
135 \\
140 \\
150\end{array}$ \\
\hline
\end{tabular}

Table 2.--Logs of test holes, seismograph shot holes, and wells--Continued

Kit Carson County--Continued

\begin{tabular}{l|c|c}
\hline & $\begin{array}{c}\text { Thickness } \\
\text { (feet) }\end{array}$ & $\begin{array}{c}\text { Depth } \\
\text { (feet) }\end{array}$ \\
\hline
\end{tabular}

$c 10-48-23 c c$

\begin{tabular}{|c|c|c|}
\hline $\begin{array}{l}\text { Ogallala formation: } \\
\text { Sand, gravel, and } \\
\text { streaks of caliche.... } \\
\text { Clay, yellow.......... } \\
\text { Pierre shale: } \\
\text { Shale, blue........... }\end{array}$ & $\begin{array}{r}155 \\
10\end{array}$ & $\begin{array}{l}155 \\
165 \\
170\end{array}$ \\
\hline \multicolumn{3}{|c|}{$\mathrm{C} 10-48-29 \mathrm{bb}$} \\
\hline 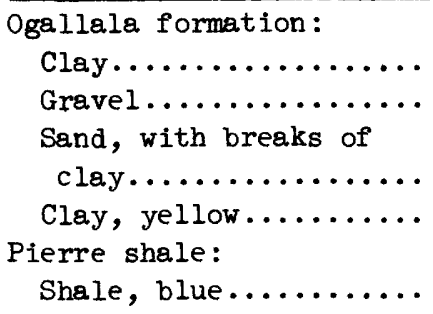 & $\begin{array}{l}50 \\
50 \\
35 \\
17\end{array}$ & $\begin{array}{r}50 \\
100 \\
135 \\
152\end{array}$ \\
\hline
\end{tabular}

C10-48-30cc

\begin{tabular}{l|r|r}
\hline Ogallala formation: & & \\
Sand, containing & & \\
streaks of gravel..... & 150 & 150 \\
Clay, yellow......... & 5 & 155 \\
Pierre shale: & & \\
Shale, blue.......... & 5 & 160 \\
\hline
\end{tabular}

C10-48-32b

\begin{tabular}{|c|c|c|}
\hline Ogallala formation: & 38 & 38 \\
\hline 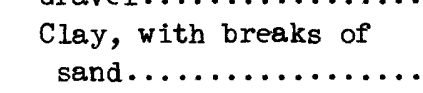 & 42 & 80 \\
\hline Grave1............. & 25 & 105 \\
\hline Clay, yellow.......... & 20 & 125 \\
\hline Pierre shale: & & \\
\hline Shale, blue.......... & 5 & 130 \\
\hline
\end{tabular}

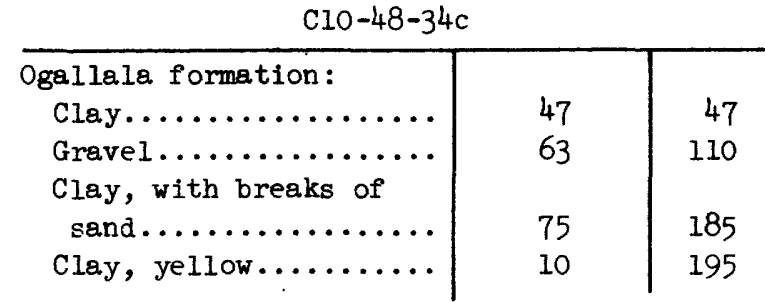


Table 2.--Logs of test holes, seismograph shot holes, and wells--Continued

Kit Carson County--Continued

\begin{tabular}{|c|c|c|}
\hline & $\begin{array}{c}\text { Thickness } \\
\text { (feet) }\end{array}$ & $\begin{array}{l}\text { Depth } \\
\text { (feet) }\end{array}$ \\
\hline \multicolumn{3}{|c|}{ c10-48-34c--Continued } \\
\hline $\begin{array}{l}\text { Pierre shale: } \\
\text { Shale, blue.......... }\end{array}$ & 5 & 200 \\
\hline \multicolumn{3}{|c|}{$c 10-49-138 a$} \\
\hline 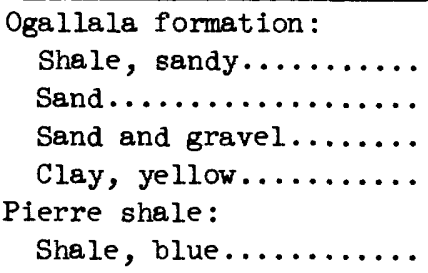 & $\begin{array}{l}30 \\
70 \\
50 \\
10\end{array}$ & $\begin{array}{r}30 \\
100 \\
150 \\
160\end{array}$ \\
\hline
\end{tabular}

C10-49-248a

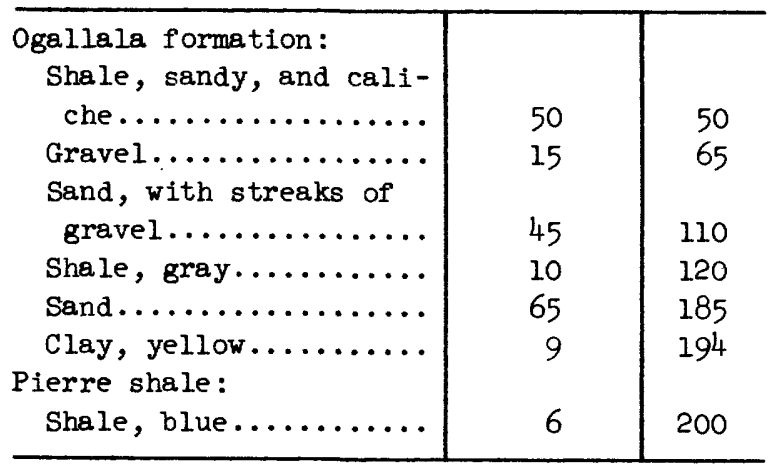

\begin{tabular}{|c|c|c|}
\hline \multicolumn{3}{|c|}{$\mathrm{C} 10-49-34 \mathrm{da}$} \\
\hline $\begin{array}{l}\text { Soil...................... } \\
\text { Ogallala formation: }\end{array}$ & 6 & 6 \\
\hline Clay and conglomerate.. & 116 & 122 \\
\hline Sand (water)........... & 8 & 130 \\
\hline Pierre shale: & & \\
\hline Shale............... & $\cdots$ & 130 \\
\hline
\end{tabular}

$\mathrm{C} 10-50-6 \mathrm{~b}$

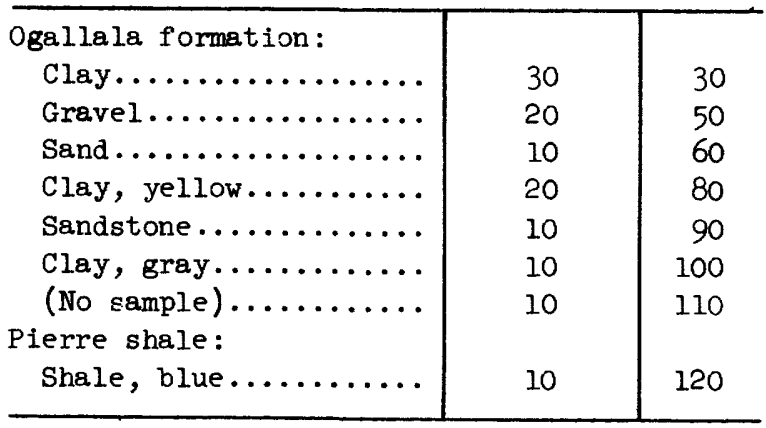

Table 2.--Logs of test holes, seismograph shot holes, and wells--Continued

Kit Carson County--Continued

\begin{tabular}{|c|c|c|}
\hline & $\begin{array}{c}\text { Thickness } \\
\text { (feet) }\end{array}$ & $\begin{array}{l}\text { Depth } \\
\text { (feet) }\end{array}$ \\
\hline \multicolumn{3}{|c|}{ C10-50-19aa } \\
\hline 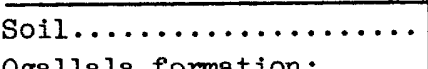 & 8 & 8 \\
\hline $\begin{array}{l}\text { Ogallala formation: } \\
\text { Gravel and clay......... }\end{array}$ & 81 & 89 \\
\hline Sand (water)........... & 11 & 100 \\
\hline Pierre shale: & & \\
\hline Shale....$\ldots \ldots \ldots \ldots$ & ... & 100 \\
\hline
\end{tabular}

\section{$\mathrm{C} 10-50-22 \mathrm{bb}$}

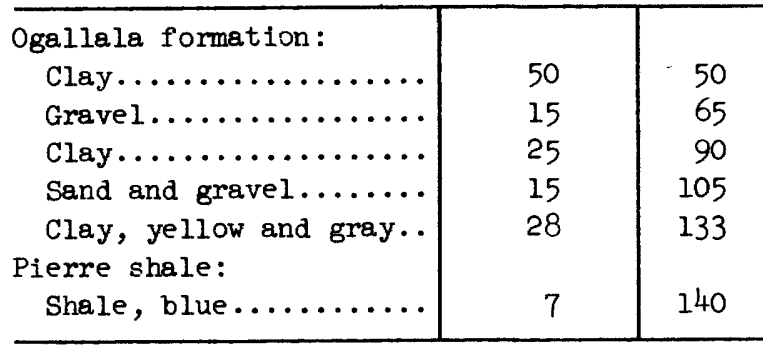

C10-51-10ab. Drilled by K. G. Wilcox, 1949

\begin{tabular}{|c|c|c|}
\hline \multirow{2}{*}{$\begin{array}{l}\text { Soil ..................... } \\
\text { Ogallala formation: }\end{array}$} & 6 & 6 \\
\hline & & \\
\hline $\begin{array}{l}\text { Sand and gravel........ } \\
\text { Caliche, soft, and }\end{array}$ & 8 & 14 \\
\hline $\begin{array}{l}\text { Caliche, soft, and } \\
\text { strips of clay........ }\end{array}$ & 14 & 28 \\
\hline Sand and gravel....... & 2 & 30 \\
\hline Sandstone...$\ldots \ldots \ldots \ldots$ & 1 & 31 \\
\hline Sand and gravel........ & 7 & 38 \\
\hline Conglomerate.......... & 1 & 39 \\
\hline Sand and gravel, loose. & 18 & 57 \\
\hline Conglome rate.......... & 6 & 63 \\
\hline Sand and gravel....... & 2 & 65 \\
\hline Conglomerate.......... & 2 & 67 \\
\hline Sand and gravel, loose. & 2 & 69 \\
\hline Pierre shale(?): & & \\
\hline Shale, yellow.......... & 2 & 71 \\
\hline
\end{tabular}

\begin{tabular}{|c|c|c|}
\hline \multicolumn{3}{|c|}{$\mathrm{Cl} 10-51-22 \mathrm{bbl}$} \\
\hline Ogallala formation: & & \\
\hline Clay...$\ldots \ldots \ldots \ldots$ & 20 & 20 \\
\hline Gravel............. & 20 & 40 \\
\hline Clay, yellow............ & 38 & 78 \\
\hline Pierre shale: & & \\
\hline Shale, blue............ & 12 & 90 \\
\hline
\end{tabular}


Table 2.--Logs of test holes, seismograph shot holes, and wells--Continued

Kit Carson County--Continued

\begin{tabular}{l|c|c}
\hline & $\begin{array}{c}\text { Thickness } \\
\text { (feet) }\end{array}$ & $\begin{array}{c}\text { Depth } \\
\text { (feet) }\end{array}$ \\
\hline
\end{tabular}

C11-41-19bc. Altitude, 4,026 feet

\begin{tabular}{|c|c|c|}
\hline Ogallala formation: & & \\
\hline Shale, sandy......... & 10 & 10 \\
\hline (No sample) .......... & 26 & 36 \\
\hline $\begin{array}{l}\text { Sand and gravel } \\
\text { streaks............ }\end{array}$ & 64 & 100 \\
\hline 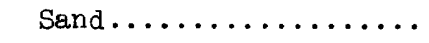 & 70 & 170 \\
\hline Shale, gray......... & 10 & 180 \\
\hline Pierre shale: & & \\
\hline Shale, blue.......... & 120 & 300 \\
\hline
\end{tabular}

C11-41-19cb. Altitude, 4,036 feet

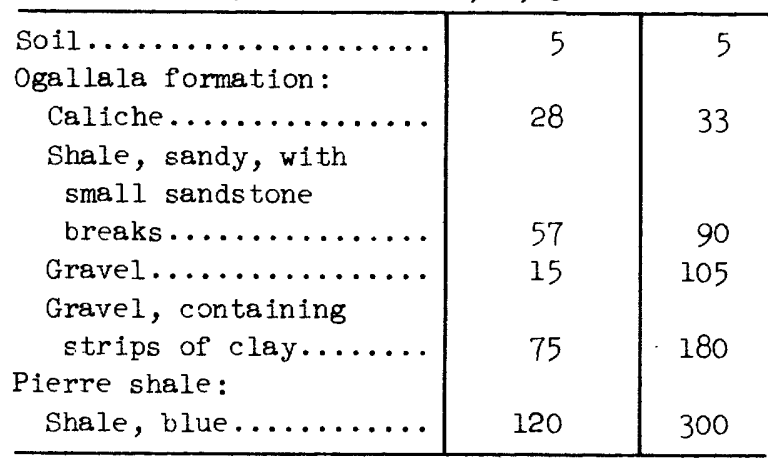

C11-41-30bb. Altitude, 4,035 feet

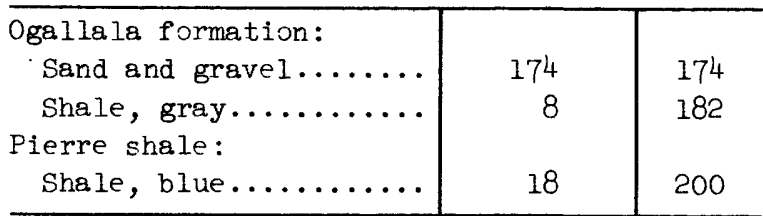

C11-42-laa. Altitude, 3,998 feet

\begin{tabular}{|c|c|c|}
\hline \multicolumn{3}{|l|}{ Ogallala formation: } \\
\hline Clay and caliche...... & 40 & 40 \\
\hline Gravel.............. & 40 & 80 \\
\hline 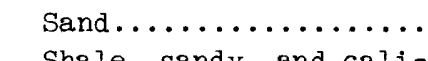 & 30 & 110 \\
\hline 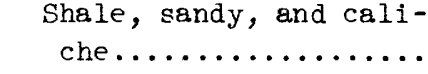 & 20 & 130 \\
\hline 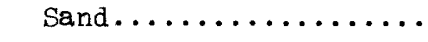 & 20 & 150 \\
\hline Clay, yellow......... & 20 & 170 \\
\hline Pierre shale: & & \\
\hline Shale, blue.......... & 10 & 180 \\
\hline
\end{tabular}

Cl1-42-38a. Altitude, 4,01l feet

\begin{tabular}{|c|c|}
\hline Ogallala formation: & 10 \\
\hline Gravel............ & 50 \\
\hline
\end{tabular}

Table 2.--Logs of test holes, seismograph shot holes, and wells--Continued

Kit Carson County--Continued

\begin{tabular}{l|c|c}
\hline & $\begin{array}{c}\text { Thickness } \\
\text { (feet) }\end{array}$ & $\begin{array}{c}\text { Depth } \\
\text { (feet) }\end{array}$ \\
\hline
\end{tabular}

C11-42-38a--Cont inued

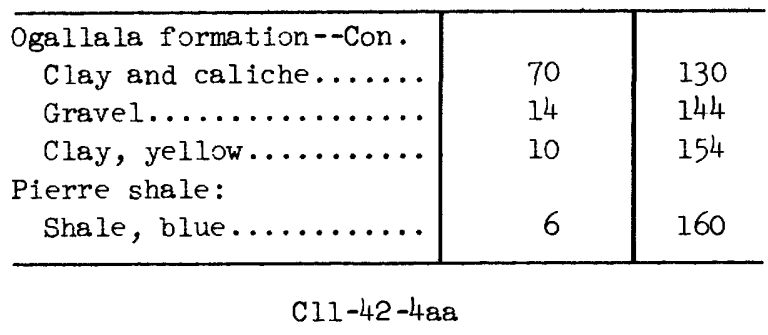

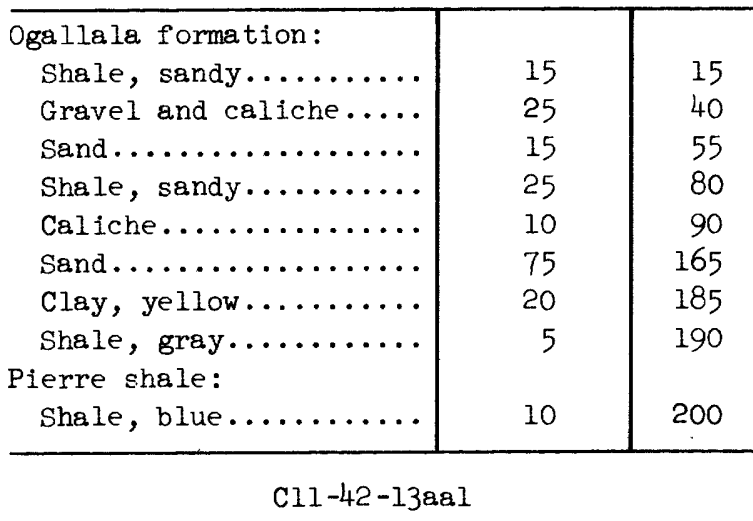

\begin{tabular}{|c|c|c|}
\hline $\begin{array}{l}\text { Soil, sandy } \ldots \ldots \ldots \ldots \ldots \\
\text { Ogallala formation: }\end{array}$ & 30 & 30 \\
\hline 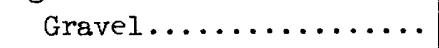 & 18 & 48 \\
\hline $\begin{array}{l}\text { Clay and caliche; con- } \\
\text { tains small breaks of } \\
\text { gravel } \ldots \ldots \ldots \ldots \ldots \ldots\end{array}$ & 72 & 120 \\
\hline $\begin{array}{l}\text { Sand and gravel } \\
\text { (water) } \ldots \ldots \ldots \ldots \ldots\end{array}$ & 40 & 160 \\
\hline Clay, gray........... & 18 & 178 \\
\hline $\begin{array}{l}\text { Pierre shale: } \\
\text { Shale, blue......... }\end{array}$ & 12 & 190 \\
\hline
\end{tabular}

\begin{tabular}{|c|c|c|}
\hline \multicolumn{3}{|c|}{ C11-42-13aa2 } \\
\hline $\begin{array}{l}\text { Soil, sandy } . . \ldots \ldots \ldots \ldots \\
\text { Ogallala formation: }\end{array}$ & 20 & 20 \\
\hline Gravel............. & 20 & 40 \\
\hline $\begin{array}{l}\text { Clay and caliche; con- } \\
\text { tains gravel breaks... }\end{array}$ & 80 & 120 \\
\hline $\begin{array}{l}\text { Sand and gravel } \\
\text { (water) } \ldots \ldots \ldots \ldots \ldots\end{array}$ & 45 & 165 \\
\hline Clay, gray........... & 15 & 180 \\
\hline Pierre shale: & & \\
\hline Shale, blue.......... & 20 & 200 \\
\hline
\end{tabular}


Table 2.--Logs of test holes, seismograph shot holes, and wells--Continued

Kit Carson County--Continued

\begin{tabular}{l|c|c}
\hline & $\begin{array}{c}\text { Thickness } \\
\text { (feet) }\end{array}$ & $\begin{array}{c}\text { Depth } \\
\text { (feet) }\end{array}$ \\
\hline
\end{tabular}

C11-42-13ad. Altitude, 4,015 feet

\begin{tabular}{l|r|r}
\hline Ogallala formation: & & \\
Sand and gravel....... & 160 & 160 \\
Shale, gray......... & 35 & 195 \\
Pierre shale: & & \\
Shale, blue......... & 15 & 210 \\
\hline
\end{tabular}

C11-42-13da. Altitude, 4,017 feet

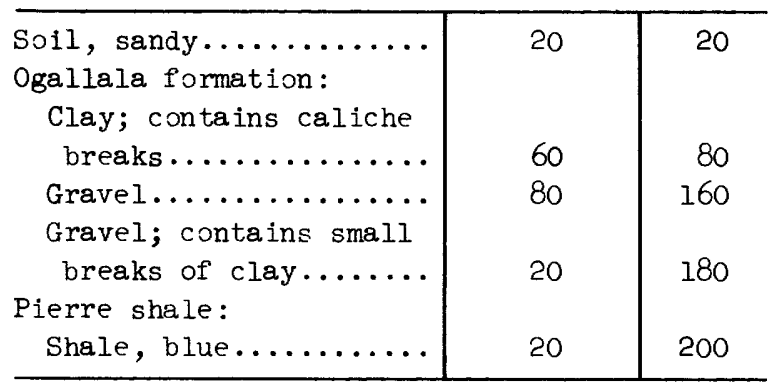

\section{Cl1-42-23aa. Altitude, 4,030 feet}

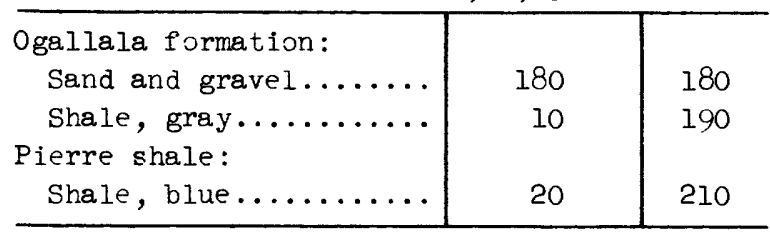

C11-42-23ad. Altitude, 4,028 feet

\begin{tabular}{|c|c|c|}
\hline Ogallala formation: & & \\
\hline Sand and gravel....... & 100 & 100 \\
\hline Sand $\ldots \ldots \ldots \ldots \ldots$ & 70 & 170 \\
\hline Shale, gray $\ldots \ldots \ldots \ldots$ & 5 & 175 \\
\hline Pierre shale: & & \\
\hline Shale, blue.......... & 25 & 200 \\
\hline
\end{tabular}

\section{C11-42-23dd}

\begin{tabular}{|c|c|c|}
\hline \multicolumn{3}{|l|}{ Ogallala formation: } \\
\hline 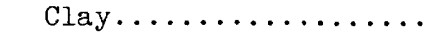 & 25 & 25 \\
\hline Caliche............ & 28 & 53 \\
\hline $\begin{array}{c}\text { Gravel, with breaks of } \\
\text { shale............. }\end{array}$ & 67 & 120 \\
\hline 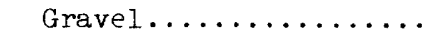 & 70 & 190 \\
\hline Shale, gray.......... & 10 & 200 \\
\hline Pierre shale: & & \\
\hline Shale, blue.......... & 30 & 230 \\
\hline
\end{tabular}

Table 2.--Logs of test holes, seismograph shot holes, and wells--Continued

Kit Carson County--Continued

\begin{tabular}{|c|c|c|}
\hline & $\begin{array}{c}\text { Thickness } \\
\text { (feet) }\end{array}$ & $\begin{array}{l}\text { Depth } \\
\text { (feet) }\end{array}$ \\
\hline \multicolumn{3}{|c|}{ C11-42-24ab. Altitude, 4,018 feet } \\
\hline \multicolumn{3}{|l|}{ Ogallala formation: } \\
\hline 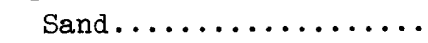 & 100 & 100 \\
\hline Sand and gravel....... & 70 & 170 \\
\hline Shale, gray.......... & 10 & 180 \\
\hline Pierre shale: & & \\
\hline Shale, blue.......... & 20 & 200 \\
\hline
\end{tabular}

C11-42-24ba. Altitude, 4,034 feet

\begin{tabular}{|c|c|c|}
\hline \\
\hline $\begin{array}{l}\text { Clay and small breaks } \\
\text { of caliche.......... }\end{array}$ & 80 & 80 \\
\hline Gravel............. & 90 & 170 \\
\hline Clay, yellow.......... & 23 & 193 \\
\hline Pierre shale: & & \\
\hline Shale, blue......... & 27 & 220 \\
\hline
\end{tabular}

\begin{tabular}{|c|c|c|}
\hline \multicolumn{3}{|c|}{$\operatorname{Cll}-42-25 a \mathrm{a}$} \\
\hline Soil................ & 4 & 4 \\
\hline Ogallala formation: & & \\
\hline Caliche............. & 21 & 25 \\
\hline $\begin{array}{c}\text { Shale, contains breaks } \\
\text { of gravel and sand- }\end{array}$ & & \\
\hline stone.............. & 95 & 120 \\
\hline 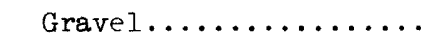 & 40 & 160 \\
\hline (No sample) ........... & 30 & 190 \\
\hline Pierre shale: & & \\
\hline Shale, blue.......... & 30 & 220 \\
\hline
\end{tabular}

C11-43-11ca. Drilled by K. G. Wilcox, 1948

\begin{tabular}{|c|c|c|}
\hline 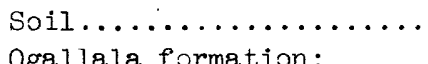 & 13 & 13 \\
\hline 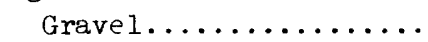 & 7 & 20 \\
\hline Sandstone........... & $\varepsilon$ & 28 \\
\hline 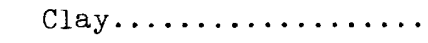 & 3 & 31 \\
\hline Caliche............. & 8 & 39 \\
\hline Gravel............. & 5 & 44 \\
\hline Caliche............. & 17 & 61 \\
\hline Clay, sandy.......... & 1 & 62 \\
\hline 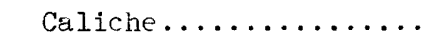 & 2 & 64 \\
\hline $\begin{array}{c}\text { Clay, sandy, and grav- } \\
\in 1 \ldots \ldots \ldots \ldots \ldots \ldots\end{array}$ & 2 & 66 \\
\hline Sandstone........... & 3 & $5^{\prime} ;$ \\
\hline Gravel............... & 3 & 12 \\
\hline
\end{tabular}


Table 2.--Logs of test holes, seismograph shot holes, and wells--Continued

Kit Carson County--Continued

\begin{tabular}{l|c|c}
\hline & $\begin{array}{c}\text { Thickness } \\
\text { (feet) }\end{array}$ & $\begin{array}{c}\text { Depth } \\
\text { (feet) }\end{array}$ \\
\hline
\end{tabular}

C11-43-11ca--Continued

\begin{tabular}{|c|c|c|}
\hline \multirow{4}{*}{ 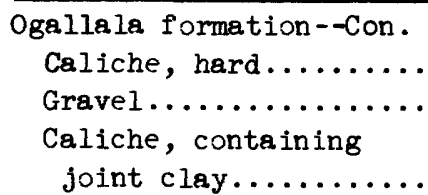 } & & \\
\hline & 3 & 75 \\
\hline & 2 & 77 \\
\hline & 4 & 81 \\
\hline Caliche and clay........ & 5 & 86 \\
\hline Grave1............. & 2 & 88 \\
\hline Conglomerate.......... & 7 & 95 \\
\hline Gravel............. & 3 & 98 \\
\hline Caliche............. & 2 & 100 \\
\hline Gravel $\ldots \ldots \ldots \ldots \ldots \ldots$ & 6 & 106 \\
\hline Rock............. & 2 & 108 \\
\hline Clay, sandy, and grav- & & \\
\hline el............. & 7 & 115 \\
\hline Clay.............. & 16 & 131 \\
\hline$(?) \ldots \ldots \ldots \ldots \ldots$ & 8 & 139 \\
\hline Clay, sandy.......... & 9 & 148 \\
\hline Sand, fine $\ldots \ldots \ldots \ldots$. & 2 & 150 \\
\hline Joint clay............ & .5 & 150.5 \\
\hline 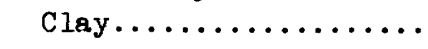 & 10.5 & 161 \\
\hline Joint clay $\ldots \ldots \ldots \ldots \ldots$ & 5 & 166 \\
\hline Gravel $\ldots \ldots \ldots \ldots \ldots \ldots$ & 12 & 178 \\
\hline Gravel and clay......... & 6 & 184 \\
\hline $\operatorname{Rock} \ldots \ldots \ldots \ldots \ldots \ldots$ & $\ldots$. & 184 \\
\hline
\end{tabular}

C11-43-12cc. Drilled by K. G. Wilcox, 1948

\begin{tabular}{|c|c|c|}
\hline \multirow{2}{*}{\multicolumn{3}{|c|}{$\begin{array}{l}\text { Soil } \ldots \ldots \ldots \ldots \ldots \ldots \ldots \\
\text { Ogallala formation: }\end{array}$}} \\
\hline & & \\
\hline Gravel, coarse......... & 32 & 47 \\
\hline Caliche, soft.......... & 4 & 51 \\
\hline Gravel, fine........... & 11 & 62 \\
\hline Caliche, soft......... & 3 & 65 \\
\hline Gravel, fine.......... & 7 & 72 \\
\hline Caliche, soft.......... & 3 & 75 \\
\hline Clay, soft, white..... & 5 & 80 \\
\hline Gravel.............. & 4 & 84 \\
\hline Conglomerate, broken... & 11 & 95 \\
\hline Clay, sandy........... & 10 & 105 \\
\hline Caliche, soft.......... & 2 & 107 \\
\hline Caliche, hard, and & & \\
\hline 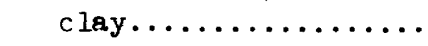 & 1 & 108 \\
\hline Rock, $\operatorname{hard} \ldots \ldots \ldots \ldots$ & 1 & 109 \\
\hline Clay, soft, red....... & 6 & 115 \\
\hline Caliche, soft, and & & \\
\hline clay............. & 3 & 118 \\
\hline Gravel........... & 5 & 123 \\
\hline
\end{tabular}

Table 2.--Logs of test holes, seismograph shot holes, and wells--Continued

Kit Carson County--Continued

\begin{tabular}{l|c|c}
\hline & $\begin{array}{c}\text { Thickness } \\
\text { (feet) }\end{array}$ & $\begin{array}{c}\text { Depth } \\
\text { (feet) }\end{array}$ \\
\hline
\end{tabular}

C11-43-12cc--Continued

\begin{tabular}{|c|c|c|}
\hline \multicolumn{3}{|l|}{ Ogallala formation--Con. } \\
\hline Conglomerate, hard..... & 5 & 128 \\
\hline Gravel, fine........ & .5 & 128.5 \\
\hline Clay, yellow.......... & 3.5 & 132 \\
\hline Caliche, hard........ & 1.5 & $133 \cdot 5$ \\
\hline Caliche, soft, and & & \\
\hline 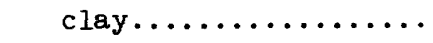 & 1 & 134.5 \\
\hline Gravel, fine......... & $3 \cdot 5$ & 138 \\
\hline Caliche, hard......... & 5 & 143 \\
\hline Gravel, fine........ & 2 & 145 \\
\hline Clay, sandy, yellow.... & 18 & 163 \\
\hline Gravel............. & 8 & 171 \\
\hline Clay ............... & 4 & 175 \\
\hline Rock............. & 3 & 178 \\
\hline Gravel............ & 7 & 185 \\
\hline Clay .............. & 4 & 189 \\
\hline Gravel and clay....... & 6 & 195 \\
\hline Gravel............ & 10 & 205 \\
\hline 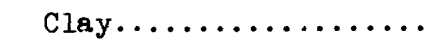 & 3 & 208 \\
\hline Rock, hard.......... & 1.5 & 209.5 \\
\hline Gravel, coarse........ & 10.5 & 220 \\
\hline Gravel and sand....... & 15 & 235 \\
\hline 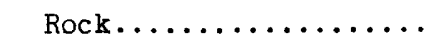 & .5 & 235.5 \\
\hline Gravel and strips of & & \\
\hline clay............... & 1.5 & 237 \\
\hline 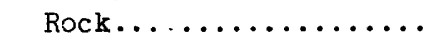 & $\ldots \ldots$ & 237 \\
\hline
\end{tabular}

C11-44-2b. Altitude, 4,268 feet

\begin{tabular}{|c|c|c|}
\hline \multicolumn{3}{|l|}{ Ogailala formation: } \\
\hline Clay...$\ldots \ldots \ldots \ldots$ & 10 & 10 \\
\hline Gravel............. & 30 & 40 \\
\hline Clay and caliche....... & 210 & 250 \\
\hline 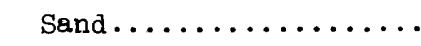 & 40 & 290 \\
\hline Clay, yellow.......... & 10 & 300 \\
\hline Pierre shale: & & \\
\hline Shale, blue......... & 10 & 310 \\
\hline
\end{tabular}

C11-45-2b. Altitude, 4,374 feet

\begin{tabular}{|c|c|c|}
\hline Ogallala formation: & & \\
\hline Clay.$\ldots \ldots \ldots \ldots \ldots$ & 10 & 10 \\
\hline Gravel............ & 8 & 18 \\
\hline Shale, sandy.......... & 32 & 50 \\
\hline 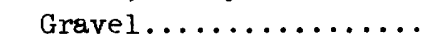 & 7 & 57 \\
\hline Shale, sandy.......... & 73 & 130 \\
\hline Sand................ & 85 & 215 \\
\hline
\end{tabular}


Table 2.--Logs of test holes, seismograph shot holes, and wells--Continued

Kit Carson County--Continued

\begin{tabular}{|c|c|c|}
\hline & $\begin{array}{c}\text { Thickness } \\
\text { (feet) }\end{array}$ & $\begin{array}{l}\text { Depth } \\
\text { (feet) }\end{array}$ \\
\hline \multicolumn{3}{|c|}{ Cll-45-2b--Continued } \\
\hline 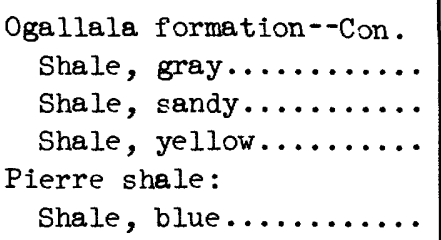 & $\begin{array}{r}10 \\
5 \\
10\end{array}$ & $\begin{array}{l}225 \\
230 \\
240 \\
250\end{array}$ \\
\hline \multicolumn{3}{|c|}{$C 11-48-5 b$} \\
\hline 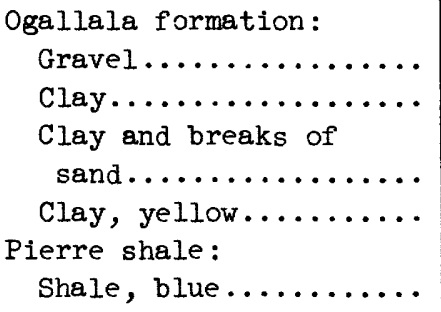 & $\begin{array}{l}20 \\
20 \\
60 \\
10 \\
10\end{array}$ & $\begin{array}{r}20 \\
40 \\
100 \\
110 \\
120\end{array}$ \\
\hline \multicolumn{3}{|l|}{$\operatorname{cll}-49-2$} \\
\hline 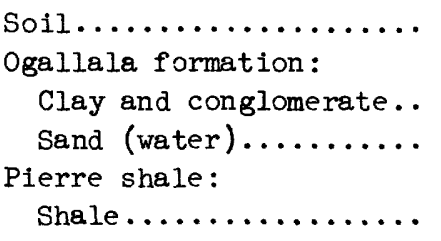 & $\begin{array}{l}6 \\
64 \\
10 \\
\ldots\end{array}$ & $\begin{array}{l}6 \\
70 \\
80 \\
80\end{array}$ \\
\hline \multicolumn{3}{|c|}{$C l 1-49-5 b b$} \\
\hline 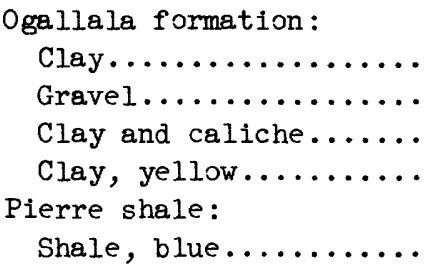 & $\begin{array}{l}20 \\
10 \\
70 \\
20 \\
10\end{array}$ & $\begin{array}{r}20 \\
30 \\
100 \\
120 \\
130\end{array}$ \\
\hline \multicolumn{3}{|c|}{$c 11-49-11 b c$} \\
\hline 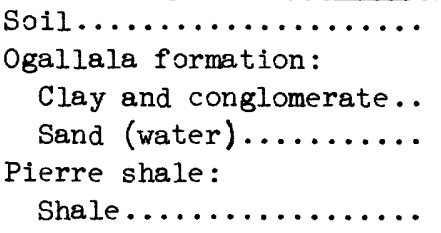 & $\begin{array}{r}142 \\
6\end{array}$ & $\begin{array}{r}6 \\
148 \\
154\end{array}$ \\
\hline
\end{tabular}

Table 2.--Logs of test holes, seismograph shot holes, and wells--Continued

Kit Carson County--Continued

\begin{tabular}{|c|c|c|}
\hline & $\begin{array}{c}\text { Thickness } \\
\text { (feet) }\end{array}$ & $\begin{array}{l}\text { Depth } \\
\text { (feet) }\end{array}$ \\
\hline \multicolumn{3}{|c|}{$C 11-49-22 a$} \\
\hline $\begin{array}{l}\text { Soil.................... } \\
\text { Ogallala formation: }\end{array}$ & 10 & 10 \\
\hline $\begin{array}{l}\text { Conglomerate and clay.. } \\
\text { Sand (water) ........... }\end{array}$ & $\begin{array}{r}129 \\
6\end{array}$ & $\begin{array}{l}139 \\
145\end{array}$ \\
\hline $\begin{array}{l}\text { Pierre shale: } \\
\text { Shale............. }\end{array}$ & $\ldots$ & 145 \\
\hline
\end{tabular}

\section{C11-50-2ba}

\begin{tabular}{|c|c|c|}
\hline $\begin{array}{l}\text { Soil ..................... } \\
\text { Ogallala formation: }\end{array}$ & 5 & 5 \\
\hline Clay and conglomerate.. & 131 & 136 \\
\hline Sand (water) ............... & 4 & 140 \\
\hline Pierre shale: & & \\
\hline Shale................. & $\cdots$ & 140 \\
\hline
\end{tabular}

\begin{tabular}{|c|c|c|}
\hline Ogallala formation: & & \\
\hline Clay...$\ldots \ldots \ldots \ldots$ & 45 & 45 \\
\hline Gravel $\ldots \ldots \ldots \ldots \ldots \ldots$ & 25 & 70 \\
\hline Clay...$\ldots \ldots \ldots \ldots$ & 25 & 95 \\
\hline Sand $\ldots \ldots \ldots \ldots \ldots \ldots$ & 20 & 115 \\
\hline Clay, yellow........... & 22 & 137 \\
\hline Pierre shale: & & \\
\hline Shale, blue........... & 13 & 150 \\
\hline
\end{tabular}

Cl1-50-32cc

\begin{tabular}{|c|c|c|}
\hline Ogallala formation: & & \\
\hline $\begin{array}{l}\text { Clay ................... } \\
\text { Sand, with breaks of }\end{array}$ & 8 & 8 \\
\hline clay $\ldots \ldots \ldots \ldots \ldots \ldots$ & 22 & 30 \\
\hline$(?) \ldots \ldots \ldots \ldots \ldots \ldots$ & 90 & 120 \\
\hline Clay, yellow............ & 27 & 147 \\
\hline Pierre shale: & & \\
\hline Shale, blue........... & 13 & 160 \\
\hline
\end{tabular}

\begin{tabular}{|c|c|c|}
\hline \multicolumn{3}{|c|}{ Cll- $-51-5 b b$} \\
\hline Ogallala formation: & & \\
\hline Clay...$\ldots \ldots \ldots \ldots$ & 8 & 8 \\
\hline Gravel.............. & 7 & 15 \\
\hline Clay, yellow........... & 53 & 68 \\
\hline Pierre shale: & & \\
\hline Shale, blue............ & 12 & 80 \\
\hline
\end{tabular}


Table 2.--Logs of test holes, seismograph shot holes, and wells--Continued

Kit Carson County--Continued

\begin{tabular}{|c|c|c|}
\hline & $\begin{array}{c}\text { Thickness } \\
\text { (feet) }\end{array}$ & $\begin{array}{l}\text { Depth } \\
\text { (feet) }\end{array}$ \\
\hline \multicolumn{3}{|c|}{$C 11-51-23 b b$} \\
\hline \multicolumn{3}{|l|}{ Ogallala formation: } \\
\hline 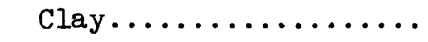 & 15 & 15 \\
\hline 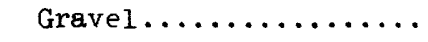 & 25 & 40 \\
\hline 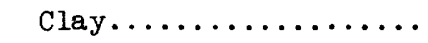 & 45 & 85 \\
\hline 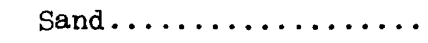 & 20 & 105 \\
\hline Clay, yellow.......... & 18 & 123 \\
\hline \multicolumn{3}{|l|}{ Pierre shale: } \\
\hline Shale, blue.......... & 7 & 130 \\
\hline
\end{tabular}

\section{Lincoln County}

\begin{tabular}{l|c|c}
\hline & $\begin{array}{c}\text { Thickness } \\
\text { (feet) }\end{array}$ & $\begin{array}{c}\text { Depth } \\
\text { (feet) }\end{array}$ \\
\hline
\end{tabular}

C7-52-27ba. Drilled by Ben Hasz, 1949

\begin{tabular}{|c|c|c|}
\hline \multirow{2}{*}{\multicolumn{3}{|c|}{$\begin{array}{l}\text { Ogallala formation: } \\
\text { (No samples) .......... }\end{array}$}} \\
\hline & 156 & 156 \\
\hline Sandstone . . . . . . . . & 6 & 162 \\
\hline Sand, tight.......... & 22 & 184 \\
\hline Sandstone and clay..... & 8 & 192 \\
\hline 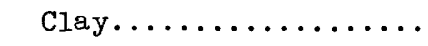 & 2 & 194 \\
\hline $\begin{array}{l}\text { Sand and gravel, with } \\
\text { some clay ........... }\end{array}$ & 22 & 216 \\
\hline 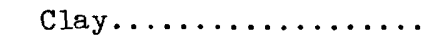 & 5 & 221 \\
\hline
\end{tabular}

C8-52-14cdl. Drilled by K. G. Wilcox, 1949

\begin{tabular}{|c|c|c|}
\hline \multirow{2}{*}{\multicolumn{3}{|c|}{$\begin{array}{l}\text { Ogallala formation: } \\
\text { (No samples).......... }\end{array}$}} \\
\hline & 70 & 70 \\
\hline Rock............... & 11 & 81 \\
\hline 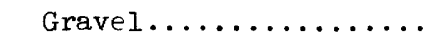 & 9 & 90 \\
\hline 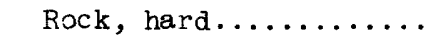 & 1.5 & $91 \cdot 5$ \\
\hline Sandstone, soft....... & $3 \cdot 5$ & 95 \\
\hline Clay, sandy.......... & 4 & 99 \\
\hline Sandstone............ & 3 & 102 \\
\hline Clay, sandy.......... & $\cdot 5$ & $102 \cdot 5$ \\
\hline $\begin{array}{l}\text { Sand and gravel........ } \\
\text { Gravel, containing }\end{array}$ & 1 & $103 \cdot 5$ \\
\hline strips of rock....... & 8.5 & 112 \\
\hline Caliche............. & 2 & 114 \\
\hline 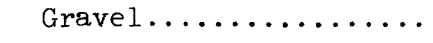 & 11.5 & 125.5 \\
\hline Conglomerate......... & .5 & 126 \\
\hline Pierre shale(?): & & \\
\hline 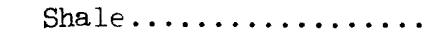 & 5 & 131 \\
\hline
\end{tabular}

Table 2.--Logs of test holes, seismograph shot holes, and wells--Continued

Lincoln County--Continued

\begin{tabular}{l|c|c}
\hline & $\begin{array}{c}\text { Thickness } \\
\text { (feet) }\end{array}$ & $\begin{array}{c}\text { Depth } \\
\text { (feet) }\end{array}$ \\
\hline
\end{tabular}

C8-52-14cd2. Drilled by K. G. Wilcox, 1949

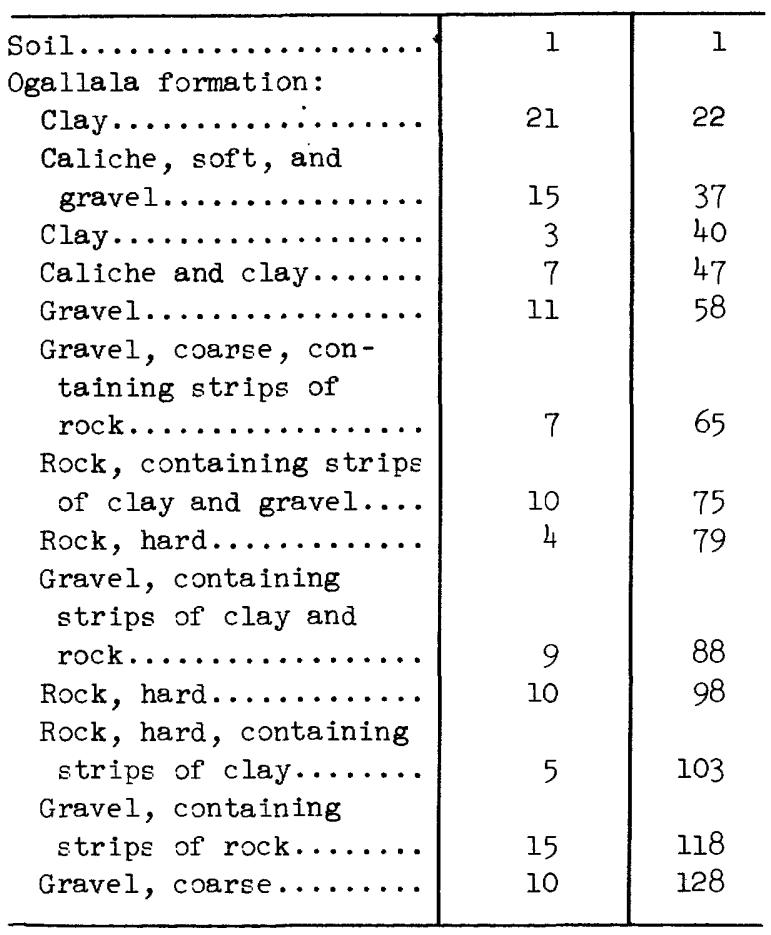

C8-52-24aa. Drilled by Ben Hasz, 1948

\begin{tabular}{|c|c|c|}
\hline $\begin{array}{l}\text { Ogallala formation: } \\
\text { (No samples).......... }\end{array}$ & 47 & 47 \\
\hline Sand, fair.......... & 8 & 55 \\
\hline Sand and clay......... & 6 & 61 \\
\hline 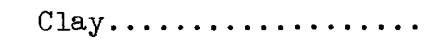 & 8 & 69 \\
\hline Clay, sandy, loose.... & 16 & 85 \\
\hline 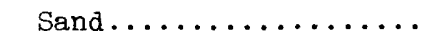 & 7 & 92 \\
\hline Clay, sandy .......... & 8 & 100 \\
\hline 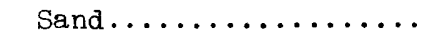 & 16 & 116 \\
\hline 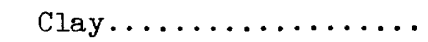 & 4 & 120 \\
\hline
\end{tabular}

C8-52-24cb. Drilled by Ben Hasz, 1948

\begin{tabular}{r|r|r}
\hline Ogallala formation. & & \\
(No samples)......... & 52 & 52 \\
Sand and sandstone..... & 4 & 56 \\
Clay, sandy, tight.... & 18 & 74 \\
Sand, clayey, lovse... & 9 & 83 \\
Clay, sandy, tight.... & 6 & 89
\end{tabular}


Table 2.--Logs of test holes, seismograph shot holes, and wells--Continued

Lincoln County--Continued

\begin{tabular}{l|c|c}
\hline & $\begin{array}{c}\text { Thickness } \\
\text { (feet) }\end{array}$ & $\begin{array}{c}\text { Depth } \\
\text { (feet) }\end{array}$ \\
\hline
\end{tabular}

$c 8-52-24 c b--C o n t$ inued

\begin{tabular}{|c|c|c|}
\hline \multicolumn{3}{|l|}{ Ogallala formation--Con. } \\
\hline Sand, loose, and clay.. & 11 & 100 \\
\hline 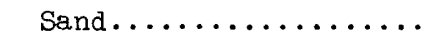 & 14 & 114 \\
\hline Sand and sandstone..... & 3 & 117 \\
\hline Clay ............. & 3 & 120 \\
\hline
\end{tabular}

C8-52-24cc. Drilled by Ben Hasz, 1948

\begin{tabular}{|c|c|c|}
\hline $\begin{array}{l}\text { Ogallala formation: } \\
\text { (No samples) } \ldots \ldots \ldots \ldots\end{array}$ & 44 & 44 \\
\hline 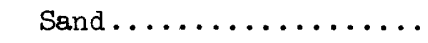 & 14 & 58 \\
\hline 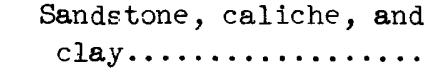 & 6 & 64 \\
\hline 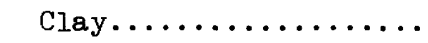 & 12 & 76 \\
\hline Clay, sandy.......... & 6 & 82 \\
\hline Sandstone............. & 7 & 89 \\
\hline Sand, tight.......... & 9 & 98 \\
\hline Sand................ & 16 & 114 \\
\hline Sandstone............ & 2 & 116 \\
\hline 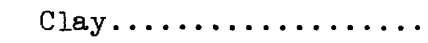 & 3 & 119 \\
\hline
\end{tabular}

C8-53-1dd. Drilled by Jack Doty, 1946

\begin{tabular}{l|r|r}
\hline $\begin{array}{l}\text { Soil and clay, sandy.... } \\
\text { Ogallala formation. }\end{array}$ & 20 & 20 \\
Sand, fine to medium... & 8 & 28 \\
Sand, containing strips & & \\
of clay............ & 9 & 37 \\
Sand and caliche...... & 3 & 40 \\
Sand, medium to coarse. & 15 & 55 \\
Sand and caliche...... & 3 & 58 \\
Sand, medium to coarse. & 7 & 65 \\
Sand and caliche..... & 6 & 71 \\
Sand, medium to coarse, & & \\
containing caliche... & 7 & 78 \\
Caliche, hard........ & 6 & 84 \\
Sand, fine to medium... & 8 & 92 \\
Clay, yellow.......... & 8 & 100 \\
\hline
\end{tabular}

C9-53-12aa. Drilled by Jack Doty, 1946

\begin{tabular}{|c|c|c|}
\hline Soil and clay, sandy..... & 19 & 19 \\
\hline Sand, fine............ & 8 & 27 \\
\hline Caliche, soft........ & 3 & 30 \\
\hline Sand, fine.......... & 8 & 38 \\
\hline 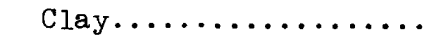 & 6 & 44 \\
\hline Sand, medium to coarse. & 8 & 52 \\
\hline
\end{tabular}

Table 2.--Logs of test holes, seismograph shot holes, and wells--Continued

Lincoln County--Continued

\begin{tabular}{l|l|l}
\hline & $\begin{array}{c}\text { Thickness } \\
\text { (feet) }\end{array}$ & $\begin{array}{c}\text { Depth } \\
\text { (feet) }\end{array}$ \\
\hline
\end{tabular}

C9-53-12aa--Continued

\begin{tabular}{|c|c|c|}
\hline 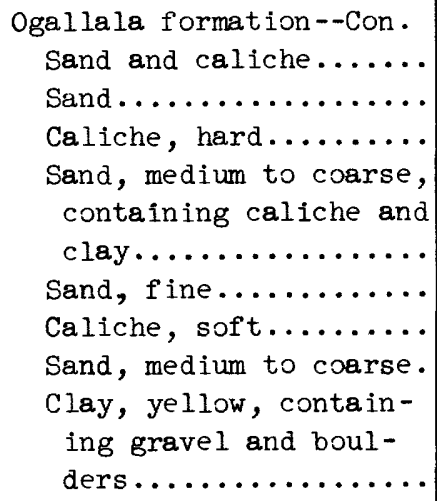 & $\begin{array}{r}8 \\
3 \\
7 \\
10\end{array}$ & $\begin{array}{l}72 \\
75 \\
82 \\
92\end{array}$ \\
\hline
\end{tabular}

C9-53-12bc. Drilled by Jack Doty, 1946

\begin{tabular}{|c|c|c|}
\hline $\begin{array}{l}\text { Soil, sand, and clay..... } \\
\text { Ogallala formation: }\end{array}$ & 24 & 24 \\
\hline 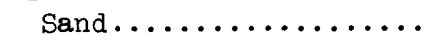 & 6 & 30 \\
\hline Caliche, soft......... & 2 & 32 \\
\hline 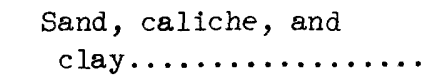 & 10 & 42 \\
\hline Sand, medium to coarse. & 6 & 48 \\
\hline Caliche, soft, brown... & 5 & 53 \\
\hline $\begin{array}{l}\text { Sand, medium to coarse. } \\
\text { Sand, fine, containing }\end{array}$ & 6 & 59 \\
\hline caliche and clay...... & 21 & 80 \\
\hline $\begin{array}{l}\text { Sandstone, brown and } \\
\text { white.............. }\end{array}$ & 5 & 85 \\
\hline Clay, yellow......... & 15 & 100 \\
\hline
\end{tabular}

C9-54-18ca. Drilled by K. G. Wilcox, 1950

Ogallala formation:

Sand, very fine to medium, clayey, tan...

Clay, firm, brown......

Sand, very fine to medium, clayey, tan... Gravel, fine to coarse, containing fine to coarse sand; $\tan . . .$. Sand, very fine to coarse, containing fine to coarse gravel with fragments of caliche and lenses of clay...............

\begin{tabular}{l|l}
3 & 3 \\
1.2 & 4.2 \\
3.3 & 7.5 \\
1 & 8.5 \\
9.5 & 18.0
\end{tabular}


Table 2.--Logs of test holes, seismograph shot holes, and wells--Continued

Lincoln County--Continued

\begin{tabular}{l|c|c}
\hline & $\begin{array}{c}\text { Thickness } \\
\text { (feet) }\end{array}$ & $\begin{array}{c}\text { Depth } \\
\text { (feet) }\end{array}$ \\
\hline
\end{tabular}

c9-54-18ca--Continued

\begin{tabular}{l|c|c}
\hline $\begin{array}{l}\text { Ogallala formation--Con. } \\
\text { Clay, firm to soft, }\end{array}$ & & \\
tan to yellowish- & & \\
tan.............. & 5 & 23 \\
$\begin{array}{c}\text { Clay, firm, gray with } \\
\text { yellow staining...... }\end{array}$ & 5 & 28 \\
$\begin{array}{l}\text { Caliche, hard, gray } \\
\text { and white........... }\end{array}$ & .5 & 28.5 \\
$\begin{array}{l}\text { Pierre shale: } \\
\text { Clay, firm, gray with } \\
\text { yellow to dark-gray } \\
\text { stains............. }\end{array}$ & & \\
\hline
\end{tabular}

Logan County

\begin{tabular}{|c|c|c|}
\hline & $\begin{array}{c}\text { Thickness } \\
\text { (feet) }\end{array}$ & $\begin{array}{l}\text { Depth } \\
\text { (feet) }\end{array}$ \\
\hline \multicolumn{3}{|c|}{$B 6-49-19 d$} \\
\hline 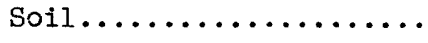 & 5 & 5 \\
\hline \multicolumn{3}{|l|}{ Ogallala formation: } \\
\hline Sand and clay......... & 55 & 60 \\
\hline Gravel.............. & 10 & 70 \\
\hline \multicolumn{3}{|l|}{ Pierre shale: } \\
\hline Shale, blue.......... & 280 & 350 \\
\hline
\end{tabular}

\begin{tabular}{|c|c|c|}
\hline \multicolumn{3}{|c|}{$B 6-50-27 c$} \\
\hline $\begin{array}{l}\text { Soil.................. } \\
\text { Ogallala formation: }\end{array}$ & 2 & 2 \\
\hline 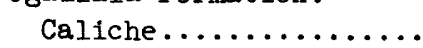 & 13 & 15 \\
\hline Gravel............ & 20 & 35 \\
\hline Shale, yellow......... & 45 & 80 \\
\hline Pierre shale: & & \\
\hline Shale, blue........... & 140 & 220 \\
\hline
\end{tabular}

\begin{tabular}{|c|c|c|}
\hline \multicolumn{3}{|c|}{$B 6-50-31 a$} \\
\hline $\begin{array}{l}\text { Soil.................. } \\
\text { Ogallala formation: }\end{array}$ & 15 & 15 \\
\hline Sand and clay, yellow.. & 120 & 135 \\
\hline Shale, yellow........ & 50 & 185 \\
\hline Quicksand and clay..... & 15 & 200 \\
\hline Pierre shale: & & \\
\hline Shale, hard.......... & . & 200 \\
\hline
\end{tabular}

Table 2.--Logs of test holes, seismograph shot holes, and wells--Continued

Logan County--Continued

\begin{tabular}{l|c|c}
\hline & $\begin{array}{c}\text { Thickness } \\
\text { (feet) }\end{array}$ & $\begin{array}{c}\text { Depth } \\
\text { (feet) }\end{array}$ \\
\hline
\end{tabular}

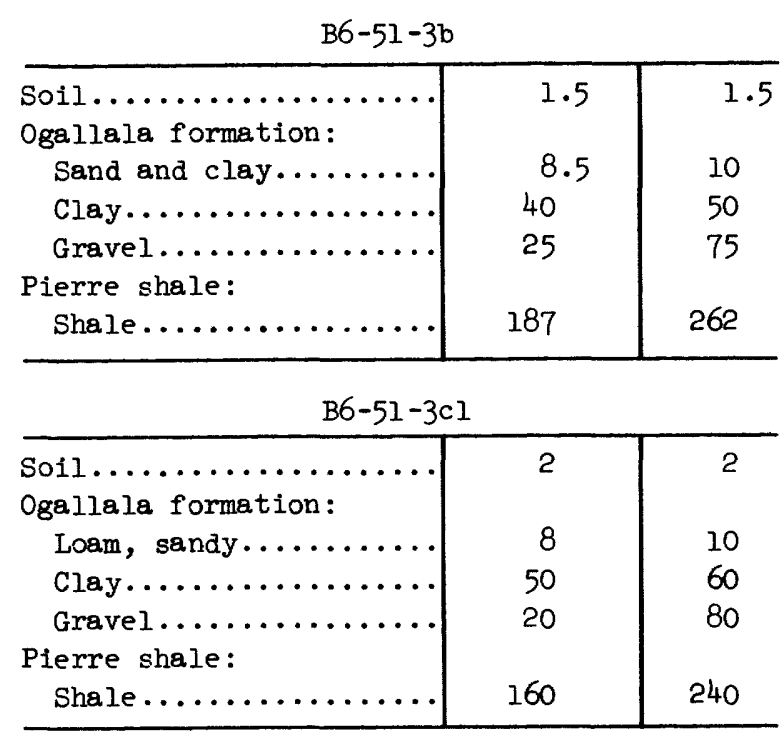

\begin{tabular}{|c|c|c|}
\hline \multicolumn{3}{|c|}{$B 6-51-3 c 2$} \\
\hline $\begin{array}{l}\text { Soil ................. } \\
\text { Ogallala formation: }\end{array}$ & 2 & 2 \\
\hline Loam, sandy........... & 8 & 10 \\
\hline Clay............... & 50 & 60 \\
\hline Gravel............ & 25 & 85 \\
\hline Pierre shale: & & \\
\hline Shale.............. & 140 & 225 \\
\hline
\end{tabular}

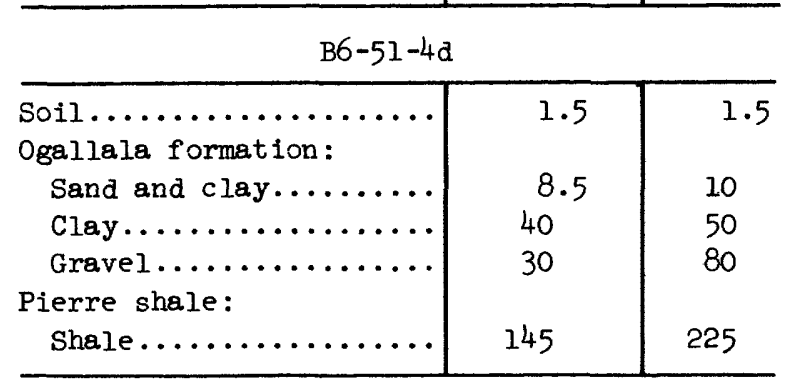

B7 $-49-7 d$

\begin{tabular}{|c|c|c|}
\hline 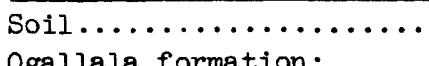 & 5 & 5 \\
\hline Sand and clay.......... & 65 & 70 \\
\hline 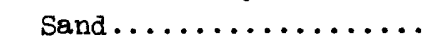 & 20 & 90 \\
\hline 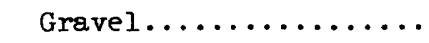 & 10 & 100 \\
\hline 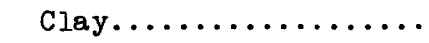 & 50 & 150 \\
\hline $\begin{array}{l}\text { Pierre shale : } \\
\text { Shale } \ldots \ldots \ldots \ldots \ldots \ldots\end{array}$ & 30 & 180 \\
\hline
\end{tabular}


Table 2.--Logs of test holes, seismograph shot holes, and wells--Continued

Logan County--Continued

\begin{tabular}{|c|c|c|}
\hline & $\begin{array}{c}\text { Thickness } \\
\text { (feet) }\end{array}$ & $\begin{array}{l}\text { Depth } \\
\text { (feet) }\end{array}$ \\
\hline \multicolumn{3}{|c|}{$B 7-49-28 d$} \\
\hline 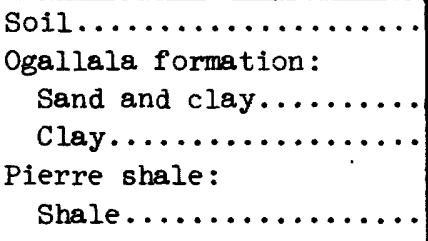 & $\begin{array}{l}6 \\
19 \\
35 \\
90\end{array}$ & $\begin{array}{r}6 \\
25 \\
60 \\
150\end{array}$ \\
\hline \multicolumn{3}{|c|}{$B 7-50-2 d$} \\
\hline 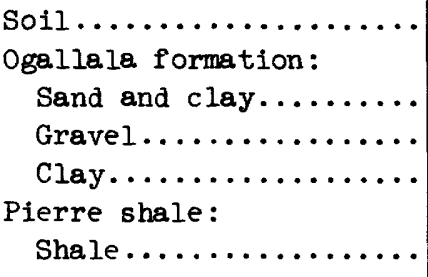 & $\begin{array}{l}5 \\
20 \\
10 \\
90 \\
60\end{array}$ & $\begin{array}{r}5 \\
25 \\
35 \\
125 \\
185\end{array}$ \\
\hline
\end{tabular}

Phillips County

\begin{tabular}{|c|c|c|}
\hline & $\begin{array}{c}\text { Thickness } \\
\text { (feet) }\end{array}$ & $\begin{array}{l}\text { Depth } \\
\text { (feet) }\end{array}$ \\
\hline B7-43-18ab. Drilled by & Arthur Hagg & $t, 1949$ \\
\hline $\begin{array}{l}\text { Soil ................... } \\
\text { Ogallala formation: }\end{array}$ & 5 & 5 \\
\hline 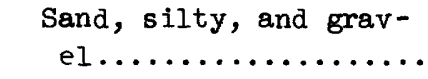 & 50 & 55 \\
\hline $\begin{array}{c}\text { Sand, gravel, clay, and } \\
\text { caliche............. }\end{array}$ & 36 & 91 \\
\hline Sand and gravel....... & 49 & 140 \\
\hline 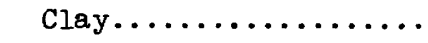 & 2 & 142 \\
\hline $\begin{array}{l}\text { Clay interbedded with } \\
\text { sand and gravel....... }\end{array}$ & 21 & 163 \\
\hline 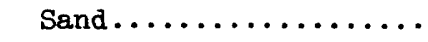 & 2 & 165 \\
\hline Sand and gravel........ & 18 & 183 \\
\hline Sand and gravel, hard.. & 9 & 192 \\
\hline Sand, silty, hard...... & 10 & 202 \\
\hline 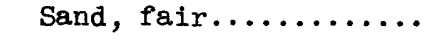 & 5 & 207 \\
\hline Sand and gravel....... & 28 & 235 \\
\hline
\end{tabular}

B7-43-33ac. Drilled by L. L. Canfield, 1941

\begin{tabular}{|c|c|c|}
\hline 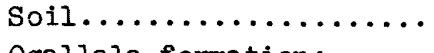 & 5 & 5 \\
\hline Ogallala formation: & & \\
\hline 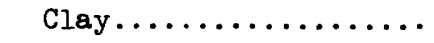 & 12 & 17 \\
\hline Clay and sand.......... & 13 & 30 \\
\hline 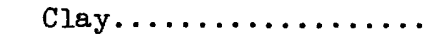 & 10 & 40 \\
\hline
\end{tabular}

Table 2.--Logs of test holes, seismograph shot holes, and wells--Continued

Phillips County--Continued

\begin{tabular}{l|c|c}
\hline & $\begin{array}{c}\text { Thickness } \\
\text { (feet) }\end{array}$ & $\begin{array}{c}\text { Depth } \\
\text { (feet) }\end{array}$ \\
\hline
\end{tabular}

B7 -43-33ac--Continued

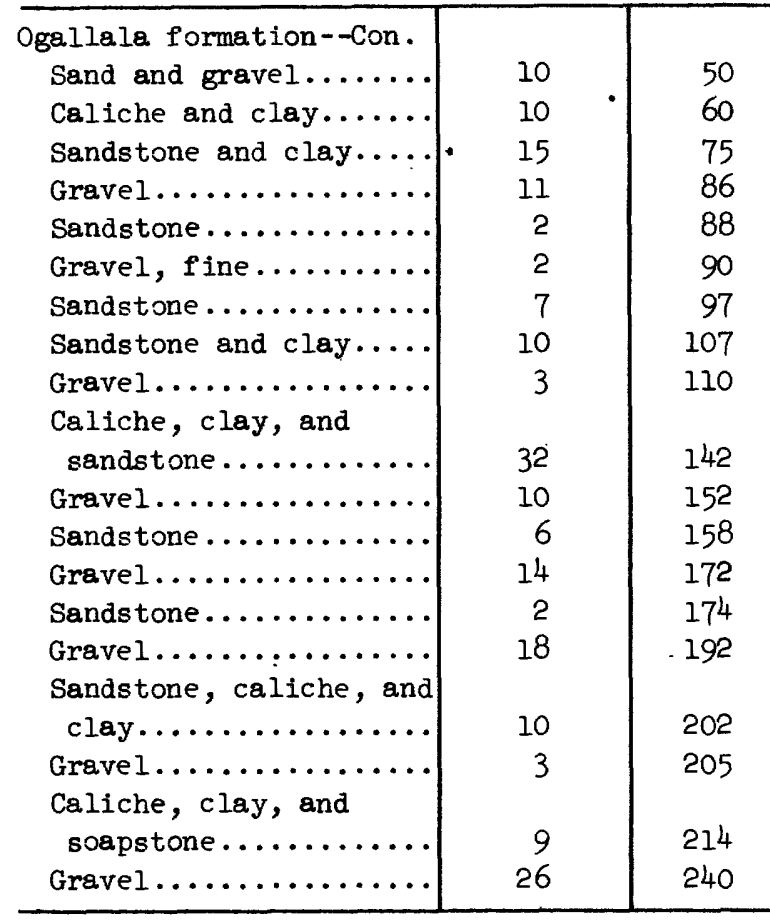

B7-44-7dd. Dug by Kelly Well Co. Inc., 1921

\begin{tabular}{|c|c|c|}
\hline Ogallala formation: & & \\
\hline (No samples)........... & 135 & 135 \\
\hline Clay and gravel........ & 6 & 141 \\
\hline Sand and gravel........ & 15 & 156 \\
\hline 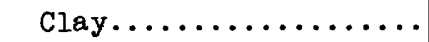 & 12 & 168 \\
\hline Gravel............. & 3 & 171 \\
\hline 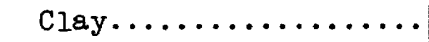 & .5 & 171.5 \\
\hline 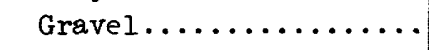 & 51 & 222.5 \\
\hline
\end{tabular}

B7-44-17bc. Dug by Kelly Well Co. Inc., 1949

\begin{tabular}{|c|c|c|}
\hline 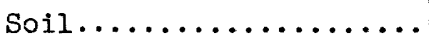 & 4 & 4 \\
\hline Ogallala formation: & & \\
\hline $\begin{array}{c}\text { Sand, containing frag- } \\
\text { ments of caliche...... }\end{array}$ & 68 & 72 \\
\hline $\begin{array}{l}\text { Clay, sandy, containing } \\
\text { fragments of caliche.. }\end{array}$ & 15 & 87 \\
\hline Clay, sandy, and grav- & & \\
\hline 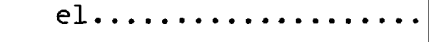 & 10 & 97 \\
\hline Sand and gravel....... & 15 & 112 \\
\hline Shale and gravel...... & 10 & 122 \\
\hline
\end{tabular}


Table 2.--Logs of test holes, seismograph shot holes, and wells--Continued

Phillips County--Continued

\begin{tabular}{l|c|c}
\hline & $\begin{array}{c}\text { Thickness } \\
\text { (feet) }\end{array}$ & $\begin{array}{c}\text { Depth } \\
\text { (feet) }\end{array}$ \\
\hline
\end{tabular}

B7 -44-17bc--Continued

\begin{tabular}{|c|c|c|}
\hline \\
\hline 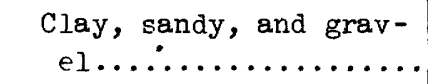 & 15 & 137 \\
\hline $\begin{array}{l}\text { Clay, sandy, and } \\
\text { shale } \ldots \ldots \ldots \ldots \ldots \ldots\end{array}$ & 10 & 147 \\
\hline $\begin{array}{l}\text { Gravel, coarse, and } \\
\text { clay............... }\end{array}$ & 47 & 194 \\
\hline $\begin{array}{l}\text { Clay, sandy, and grav- } \\
\text { el................ }\end{array}$ & 18 & 212 \\
\hline Clay and gravel....... & 51.5 & 263.5 \\
\hline
\end{tabular}

\begin{tabular}{|c|c|c|}
\hline \multicolumn{3}{|c|}{$B 7-47-5$} \\
\hline $\begin{array}{l}\text { Loam, black............ } \\
\text { Ogallala formation: }\end{array}$ & 1.5 & 1.5 \\
\hline Caliche............. & 70.5 & 72 \\
\hline 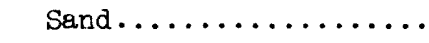 & 100 & 172 \\
\hline Rock, hard.......... & 6 & 178 \\
\hline Clay, red........... & 12 & 190 \\
\hline Shale, yellow (water).. & 100 & 290 \\
\hline Pierre shale: & & \\
\hline Shale, blue.......... & 116 & 406 \\
\hline Mud, black.......... & 70 & 476 \\
\hline Clay, flaky......... & 74 & 550 \\
\hline
\end{tabular}

B8-47-2lcc. Dug by Kelly Well Co. Inc., 1921

\begin{tabular}{|c|c|c|}
\hline \multicolumn{3}{|l|}{ Ogallala formation: } \\
\hline $\begin{array}{l}\text { Sand and gravel, } \\
\text { coarse, slightly ce- }\end{array}$ & & \\
\hline mented ............. & 150 & 150 \\
\hline 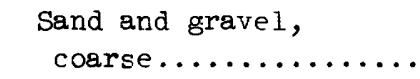 & 3 & 153 \\
\hline 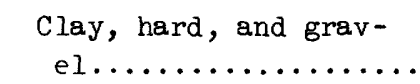 & 14 & 167 \\
\hline Sand, fine.......... & 4 & 171 \\
\hline $\begin{array}{l}\text { Gravel, cemented...... } \\
\text { Sand, loose, and cob-. }\end{array}$ & 1 & 172 \\
\hline 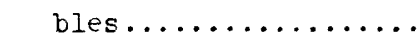 & 3 & 175 \\
\hline Sand, fine.......... & 4 & 179 \\
\hline Gravel, . edium........ & 10 & 189 \\
\hline Gravel, cemented....... & 2 & 191 \\
\hline Sand, coarse......... & 11.5 & $202 \cdot 5$ \\
\hline Clay, hard, and rocks.. & 3.5 & 206 \\
\hline 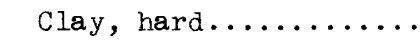 & 27 & 233 \\
\hline
\end{tabular}

Table 2.--Logs of test holes, seismograph shot holes, and wells--Continued

Phillips County--Continued

\begin{tabular}{l|c|c}
\hline & $\begin{array}{c}\text { Thickness } \\
\text { (feet) }\end{array}$ & $\begin{array}{c}\text { Depth } \\
\text { (feet) }\end{array}$ \\
\hline
\end{tabular}

B8-47-29aa. Drilled by L. L. Canfield, 1948

Ogallala formation:
Gravel, fine to medium,
pink, gray, and black
mottled; contains
sand and silt........
Silt loam, sandy, mica-
ceous, buff -tan; con-
tains roots and organ-
ic matter in upper
section..............

Sand, coarse, pink and gray, containing grav-

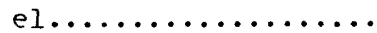

Sand, fine to coarse, and silt, buff-brown; contains gravel and clay............... Caliche, sandy, calcareous, porous, friable, buff........

Clay, sandy, calcareous, weakly compacted, friable, buff; contains a small amount of fine to medium gravel and a few calcareous concretions as much as I inch in diameter.... Caliche, sandy, slightly plastic, lightbrown.............

Gravel, calcareous, tangray, containing medium to coarse sand and pebbles............

Clay, sandy, firm, light-gray, containing medium to coarse sand.

Caliche, firm, friable, buff-gray, containing minor amount of medium

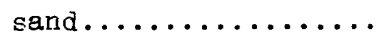
Sand, slightly silty, argillaceous, friable to loose, gray; contains some gravel.... Caliche, sandy, friable, light-gray......

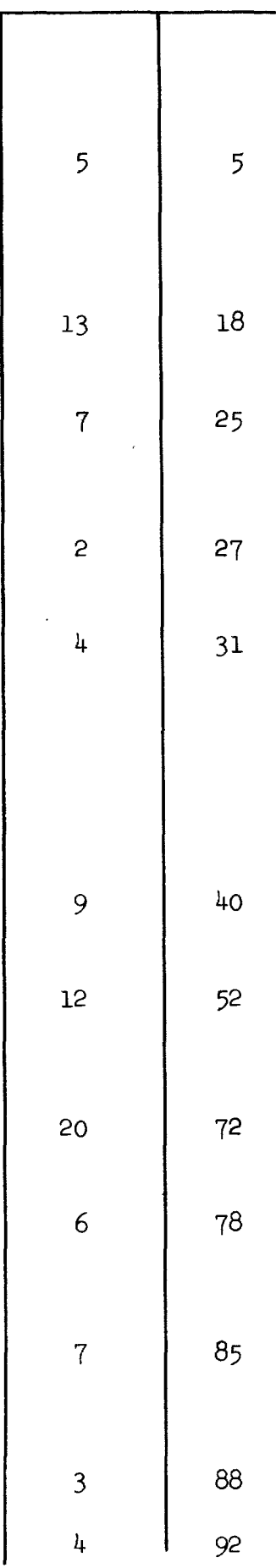


Table 2.--Logs of test holes, seismograph shot holes, and wells--Continued

Phillips County--Continued

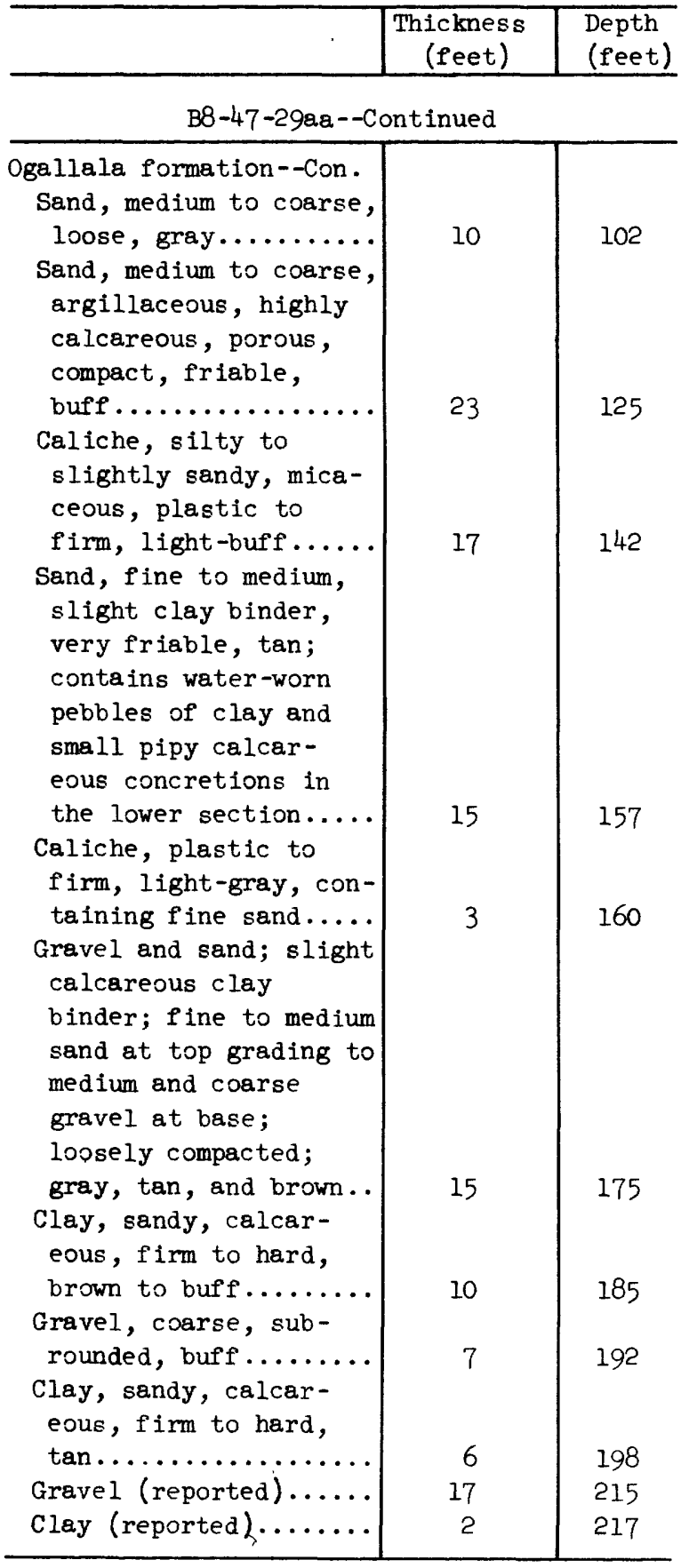

Table 2.--Logs of test holes, seismograph shot holes, and wells--Continued

Sedgwick County

\begin{tabular}{l|c|c}
\hline & $\begin{array}{c}\text { Thickness } \\
\text { (feet) }\end{array}$ & $\begin{array}{c}\text { Depth } \\
\text { (feet) }\end{array}$ \\
\hline
\end{tabular}

B10-44-35ca. Drilled by Ellithorpe and Putnam, 1949

\begin{tabular}{|c|c|c|}
\hline 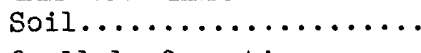 & 12 & 12 \\
\hline Ogallala formation: & & \\
\hline Gravel.............. & 21 & 33 \\
\hline 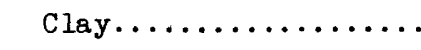 & 15 & 48 \\
\hline 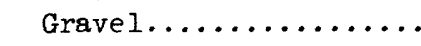 & 47.5 & 95 . \\
\hline Cobbles............... & .5 & 96 \\
\hline Gravel, cemented...... & 37 & 133 \\
\hline Gravel, loose.......... & 6 & 139 \\
\hline $\begin{array}{l}\text { Sand, containing strips } \\
\text { of caliche........... }\end{array}$ & 14 & 153 \\
\hline $\begin{array}{l}\text { Gravel, containing } \\
\text { strips of caliche.... }\end{array}$ & 30 & 183 \\
\hline Clay...$\ldots \ldots \ldots \ldots$ & 15 & 198 \\
\hline $\begin{array}{l}\text { Gravel, containing } \\
\text { strips of caliche.... }\end{array}$ & 2 & 200 \\
\hline Caliche............. & 2 & 202 \\
\hline Gravel, coarse, loose.. & 16 & 218 \\
\hline $\begin{array}{l}\text { Clay } . . . \ldots \ldots \ldots \ldots \\
\text { Gravel, coarse, fairly }\end{array}$ & 4 & 222 \\
\hline 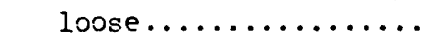 & 21 & 243 \\
\hline Gravel, coarse, red, & & \\
\hline $\begin{array}{l}\text { black, and white....... } \\
\text { Gravel, containing }\end{array}$ & 22 & 265 \\
\hline $\begin{array}{l}\text { strips of caliche..... } \\
\text { Gravel, interbedded }\end{array}$ & 8 & 273 \\
\hline with caliche......... & 15 & 288 \\
\hline Gravel, loose......... & 15 & 303 \\
\hline $\begin{array}{l}\text { Gravel, loose, con- } \\
\text { taining some clay..... }\end{array}$ & 28 & 331 \\
\hline Clay, hard............ & 17 & 348 \\
\hline
\end{tabular}

B10-44-35db. Drilled by Ellithorpe and Putnam, 1949

\begin{tabular}{|c|c|c|}
\hline Soil........................ & 12 & 12 \\
\hline Ogallala formation: & & \\
\hline 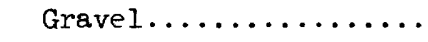 & 28 & 40 \\
\hline 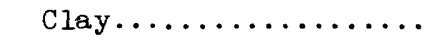 & 15 & 55 \\
\hline 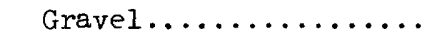 & - $\quad 34$ & 89 \\
\hline 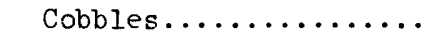 & 3 & 92 \\
\hline 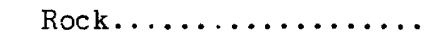 & 1 & 93 \\
\hline
\end{tabular}


Table 2.--Logs of test holes, seismograph shot holes, and wells--Continued

Sedgwick County--Continued

\begin{tabular}{|c|c}
\hline $\begin{array}{c}\text { Thickness } \\
\text { (feet) }\end{array}$ & $\begin{array}{c}\text { Depth } \\
\text { (feet) }\end{array}$ \\
\hline
\end{tabular}

B10-44-35db--Continued

\begin{tabular}{|c|c|c|}
\hline \multicolumn{3}{|l|}{ Ogallala formation--Con. } \\
\hline 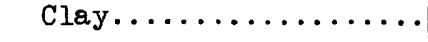 & 22 & 115 \\
\hline Clay, very hard....... & 5 & 120 \\
\hline 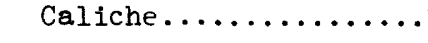 & 13 & 133 \\
\hline Gravel, cemented...... & 17 & 150 \\
\hline Clay, pink........... & 17 & 167 \\
\hline Clay, hard, in strips.. & 30 & 197 \\
\hline Clay, sandy, coarse, & & \\
\hline firm................... & 30 & 227 \\
\hline 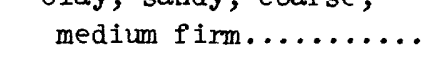 & 24 & 251 \\
\hline 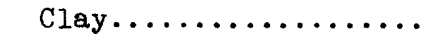 & 6 & 257 \\
\hline $\begin{array}{l}\text { Gravel, coarse, and } \\
\text { medium firm clay...... }\end{array}$ & 15 & 272 \\
\hline 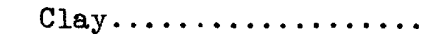 & 15 & 287 \\
\hline $\begin{array}{l}\text { Gravel, coarse, con- } \\
\text { taining medium firm }\end{array}$ & & \\
\hline clay............... & 36 & 323 \\
\hline 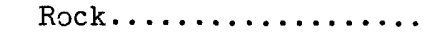 & 7 & 330 \\
\hline $\begin{array}{l}\text { Sand, coarse, grading } \\
\text { to medium gravel...... }\end{array}$ & 17 & 347 \\
\hline $\begin{array}{l}\text { Gravel, coarse, con- } \\
\text { taining medium firm }\end{array}$ & & \\
\hline 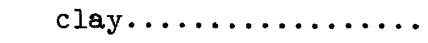 & 30 & 377 \\
\hline Sand, cemented........ & 30 & 407 \\
\hline
\end{tabular}

Washington County

\begin{tabular}{l|l|l}
\hline & $\begin{array}{c}\text { Thickness } \\
\text { (feet) }\end{array}$ & $\begin{array}{c}\text { Depth } \\
\text { (feet) }\end{array}$ \\
\hline
\end{tabular}

B1 $-51-11 b$

\begin{tabular}{|c|c|c|}
\hline 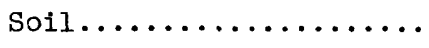 & 2 & 2 \\
\hline Ogallala formation: & & \\
\hline Gravel................ & 7 & 9 \\
\hline Rock and gravel....... & 251 & 260 \\
\hline Soapstone............ & 6 & 266 \\
\hline 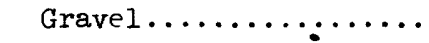 & 33 & 299 \\
\hline Pierre shale: & & \\
\hline Shale, blue.......... & 1 & 300 \\
\hline
\end{tabular}

$B 2-52-2 b$

\begin{tabular}{|c|c|c|}
\hline 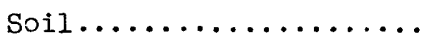 & 25 & 25 \\
\hline Ogallala formation: & & \\
\hline Grave1, cemented, hard. & 25 & 50 \\
\hline 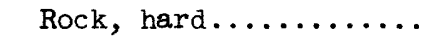 & 20 & 70 \\
\hline
\end{tabular}

Table 2.--Logs of test holes, seismograph shot holes, and wells--Continued

Washington County--Continued

\begin{tabular}{l|c|c}
\hline & $\begin{array}{c}\text { Thickness } \\
\text { (feet) }\end{array}$ & $\begin{array}{c}\text { Depth } \\
\text { (feet) }\end{array}$ \\
\hline
\end{tabular}

B2 $-52-2 b--C o n t i n u e d$

\begin{tabular}{|c|c|c|}
\hline \multicolumn{3}{|l|}{ Ogallala formation--Con. } \\
\hline Sandstone............ & 8 & 78 \\
\hline Sandstone, soft....... & 4 & 82 \\
\hline Rock, hard.......... & 3 & 85 \\
\hline $\begin{array}{l}\text { Sandstone and caliche.. } \\
\text { Sandstone and brown }\end{array}$ & 15 & 100 \\
\hline shale.............. & 58 & 158 \\
\hline Pierre shale: & & \\
\hline Shale.............. & 27 & 185 \\
\hline
\end{tabular}

B2-52-8aadc 1. Drilled by Chicago Burlington \& Quincy Railroad Co., 1919

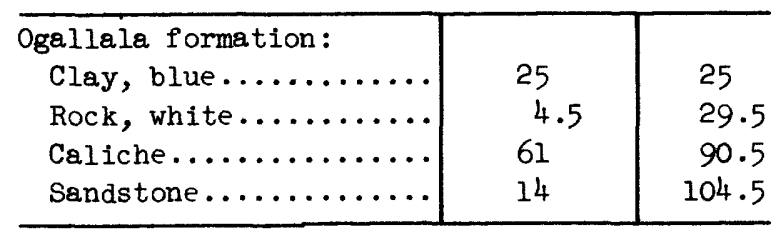

B2-52-8aadc2. Drilled by Chicago Burlington \& Quincy Railroad Co., 1919

\begin{tabular}{|c|c|c|}
\hline 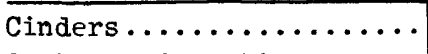 & 1.5 & 1.5 \\
\hline Ogellala formation: & & \\
\hline Clay, blue.......... & 25 & 26.5 \\
\hline Caliche.............. & $57 \cdot 5$ & 84 \\
\hline Caliche, very hard..... & 16.5 & 100.5 \\
\hline
\end{tabular}

B2-52-8abdc. Drilled by Chicago Burlington \& Quincy Railroed Co., 1919

\begin{tabular}{|c|c|c|}
\hline 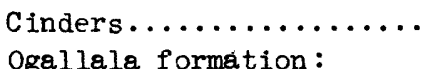 & 1 & 1 \\
\hline Clay, blue........... & 11 & 12 \\
\hline Caliche............ & 13 & 25 \\
\hline Rock, white......... & 4.5 & 29.5 \\
\hline 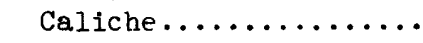 & 43 & $72 \cdot 5$ \\
\hline Rock, white.......... & $\begin{array}{l}7 \cdot 3 \\
6\end{array}$ & $\begin{array}{l}79.8 \\
85.8\end{array}$ \\
\hline
\end{tabular}

B2-52-8adb. Drilled by Chicago Burlington \& Quincy Railroad Co., 1917

\begin{tabular}{|c|c|c|}
\hline Ogallala formation: & & \\
\hline Clay .............. & 26 & 26 \\
\hline Clay, sandy.......... & 49 & 75 \\
\hline 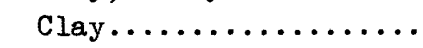 & 5 & 80 \\
\hline Sand, coarse......... & 8 & 88 \\
\hline Pierre shale (?): & 14 & 102 \\
\hline
\end{tabular}


Table 2.--Logs of test holes, seismograph shot holes, and wells--Continued

Washington County--Continued

\begin{tabular}{|c|c|c|}
\hline & $\begin{array}{c}\text { Thickness } \\
\text { (feet) }\end{array}$ & $\begin{array}{l}\text { Depth } \\
\text { (feet) }\end{array}$ \\
\hline \multicolumn{3}{|c|}{$\begin{array}{l}\text { B2-52-8bbadc. Drilled by Chicago Burlington } \\
\text { \& Quincy Railroad Co., } 1919\end{array}$} \\
\hline 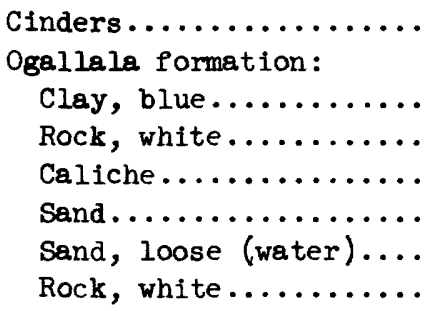 & $\begin{array}{c}24 \\
4.5 \\
52 \\
4 \\
3 \\
\ldots\end{array}$ & $\begin{array}{l}25 \\
29.5 \\
81.5 \\
85.5 \\
88.5 \\
88.5\end{array}$ \\
\hline
\end{tabular}

B2-52-8bbdc. Drilled by Chicago Burlington \& Quincy Railroad Co., 1919

\begin{tabular}{|c|c|c|}
\hline Ogallala formation: & & \\
\hline Clay...$\ldots \ldots \ldots \ldots$ & 38 & 38 \\
\hline Caliche, hard.......... & 2 & 40 \\
\hline Sand and gravel........ & 20 & 60 \\
\hline Gravel, cemented...... & 14 & 74 \\
\hline Clay and sand......... & 6 & 80 \\
\hline Sand and sandstone..... & 30 & 110 \\
\hline Limestone............ & 20 & 130 \\
\hline Limestone, brown........ & 8 & 138 \\
\hline $\begin{array}{l}\text { Joint clay and yellow } \\
\text { clay } \ldots \ldots \ldots \ldots \ldots \ldots\end{array}$ & 27 & 165 \\
\hline Caliche, yellow....... & 60 & 225 \\
\hline Pierre shale: & & \\
\hline Shale, gray............ & 115 & 340 \\
\hline Sandstone...$\ldots \ldots \ldots$ & 10 & 350 \\
\hline Shale, gray $\ldots \ldots \ldots \ldots$ & 110 & 460 \\
\hline Sandstone...$\ldots \ldots \ldots$ & 15 & 475 \\
\hline Sandstone and shale.... & 25 & 500 \\
\hline Shale, gray, and sand.. & 340 & 840 \\
\hline Sandstone............. & 4 & 844 \\
\hline Shale, gray.......... & 101 & 945 \\
\hline Sandstone, containing & & \\
\hline oil stain...$\ldots \ldots \ldots$ & 2 & 947 \\
\hline Shale, gray........... & 583 & 1,530 \\
\hline Shale, granulated...... & 12 & 1,542 \\
\hline Shale, gray........... & 141 & 1,683 \\
\hline
\end{tabular}

B2-52-8bcbc. Drilled by Chicago Burlington \& Quincy Railroad Co., 1919

Ogallala formation:

Clay, blue...........

Caliche.............

Gravel, pink, and soil.
Table 2.--Logs of test holes, seismograph shot holes, and wells--Continued

Washington County--Continued

\begin{tabular}{l|l|l}
\hline & $\begin{array}{c}\text { Thickness } \\
\text { (feet) }\end{array}$ & $\begin{array}{c}\text { Depth } \\
\text { (feet) }\end{array}$ \\
\hline
\end{tabular}

B2 $-52-8 b c b c--C o n t i n u e d$

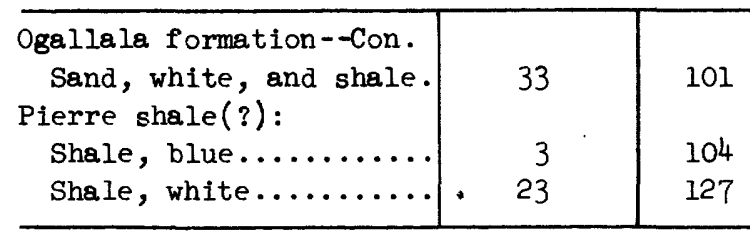

B2-52-8cdac. Drilled by Chicago Burlington \& Quincy Railroad Co., 1919

\begin{tabular}{|c|c|c|}
\hline Cinders...$\ldots \ldots \ldots \ldots$ & 2 & 2 \\
\hline Ogallala formation: & & \\
\hline Clay, blue........... & 23 & 25 \\
\hline Caliche............ & 49 & 74 \\
\hline 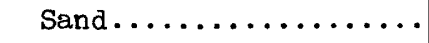 & 12 & 86 \\
\hline
\end{tabular}

B2-52-8dbadc. Drilled by Chicago Burlington \& Quincy Railroad Co., 1919

\begin{tabular}{|c|c|c|}
\hline Cinders.$\ldots \ldots \ldots \ldots \ldots \ldots$ & 1 & 1 \\
\hline Ogallala formation: & & \\
\hline Clay, blue........... & $19 \cdot 5$ & 20.5 \\
\hline Rock, white.......... & $4 \cdot 5$ & 25 \\
\hline 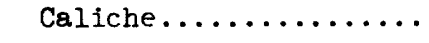 & 27 & 52 \\
\hline Shale, white......... & 7 & 59 \\
\hline Clay and gravel....... & 51 & 110 \\
\hline
\end{tabular}

B2-52-8dbc. Drilled by Chicago Burlington \& Quincy Railroad Co., 1919

\begin{tabular}{|c|c|c|}
\hline $\begin{array}{l}\text { Cinders } \ldots \ldots \ldots \ldots \ldots \ldots \\
\text { Ogallala formation: }\end{array}$ & 3 & 3 \\
\hline Clay, blue............ & 10 & 13 \\
\hline Caliche............. & 68 & 81 \\
\hline Sand, fine (water)..... & 6 & 87 \\
\hline $\begin{array}{l}\text { Sand, medium (water)... } \\
\text { Pierre shale (?): }\end{array}$ & 5 & 92 \\
\hline Shale.............. & $\ldots$ & 92 \\
\hline
\end{tabular}

B2-52-8dcac. Drilled by Chicago Burlington \& Quincy Railroad Co., 1919

\begin{tabular}{|c|c|c|}
\hline Cinders.............. & 1 & 1 \\
\hline Ogallala formation: & 24 & 25 \\
\hline $\begin{array}{l}\text { Clay, blue........... } \\
\text { Rock, white........... }\end{array}$ & 4.5 & 29.5 \\
\hline 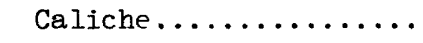 & 52 & 81.5 \\
\hline Sand, medium (water)... & 11 & $92 \cdot 5$ \\
\hline
\end{tabular}


Table 2.--Logs of test holes, seismograph shot holes, and wells--Continued

Washington County--Continued

\begin{tabular}{l|l|l}
\hline & $\begin{array}{c}\text { Thickness } \\
\text { (feet) }\end{array}$ & $\begin{array}{c}\text { Depth } \\
\text { (feet) }\end{array}$ \\
\hline
\end{tabular}

B2 $-52-8 d c b c$. Drilled by Chicago Burlington \& Quincy Railroad Co., 1919

\begin{tabular}{|c|c|c|}
\hline $\begin{array}{l}\text { Ogallala formation: } \\
\text { Clay, blue ........... }\end{array}$ & 24 & 24 \\
\hline Rock, white....... & 4.5 & 28.5 \\
\hline 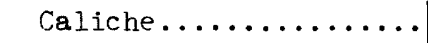 & $47 \cdot 5$ & 76 \\
\hline Shale, white........ & 30 & 106 \\
\hline Caliche............ & 14 & 120 \\
\hline
\end{tabular}

B2-52-8ddac. Drilled by Chicago Burlington \& Quincy Railroad Co., 1919

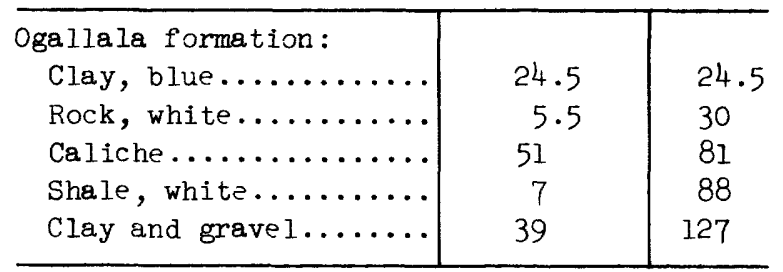

$B 2-52-9 b$

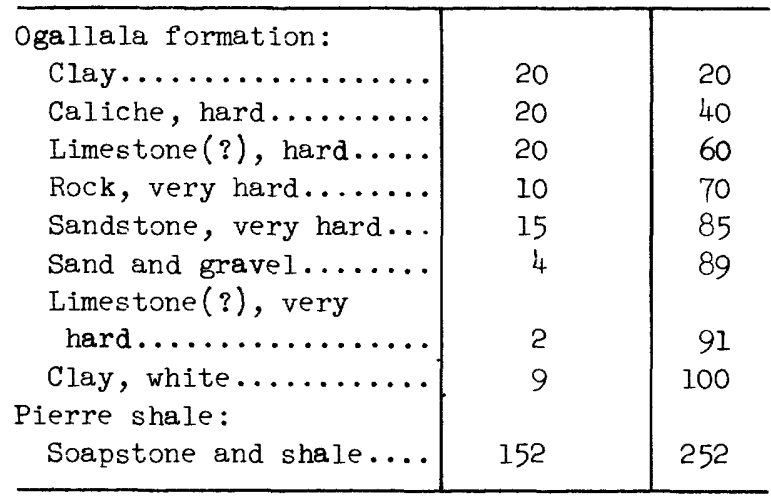

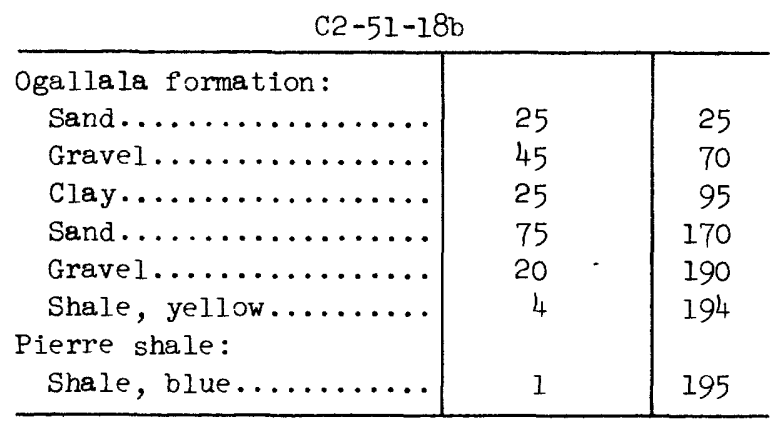

$C 4-51-7 d$

\begin{tabular}{|c|c|c|}
\hline Ogallala formation: & & \\
\hline 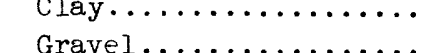 & 40 & $\begin{array}{l}40 \\
55\end{array}$ \\
\hline
\end{tabular}

Table 2.--Logs of test holes, seismograph shot holes, and wells--Continued

Washington County--Continued

\begin{tabular}{|c|c|c|}
\hline & $\begin{array}{c}\text { Thickness } \\
\text { (feet) }\end{array}$ & $\begin{array}{l}\text { Depth } \\
\text { (feet) }\end{array}$ \\
\hline \multicolumn{3}{|c|}{ C4-5I-7d--Continued } \\
\hline \multicolumn{3}{|l|}{ Ogallala formation--Con. } \\
\hline Clay and gravel....... & 5 & 60 \\
\hline Soapstone........... & 3 & 63 \\
\hline Gravel............. & 7 & 70 \\
\hline \multicolumn{3}{|l|}{ Pierre shale: } \\
\hline 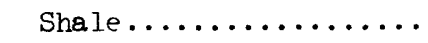 & 1 & 71 \\
\hline
\end{tabular}

C5-49-4bb. Drilled by L. L. Canfield, 1950

\begin{tabular}{|c|c|c|}
\hline $\begin{array}{l}\text { Soil ............... } \\
\text { Ogallala formation: }\end{array}$ & 3 & 3 \\
\hline 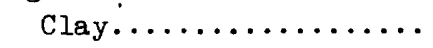 & 2 & 5 \\
\hline Sand and gravel....... & 6 & 11 \\
\hline 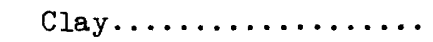 & 5 & 16 \\
\hline 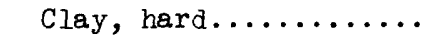 & 4 & 20 \\
\hline 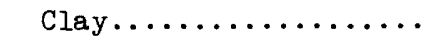 & 5 & 25 \\
\hline Gravel, solid........ & 12 & 37 \\
\hline 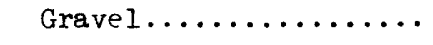 & 19 & 56 \\
\hline 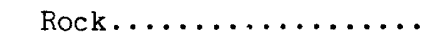 & 2 & 58 \\
\hline $\begin{array}{l}\text { Gravel and clay........ } \\
\text { Sand, gravel, and }\end{array}$ & 4 & 62 \\
\hline 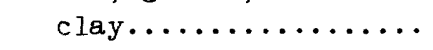 & 14 & 76 \\
\hline Pierre shale: & & \\
\hline Shale............... & 4 & 80 \\
\hline \multicolumn{3}{|c|}{ Yuma County } \\
\hline & $\begin{array}{l}\text { ckness } \\
\text { feet) }\end{array}$ & $\begin{array}{l}\text { Depth } \\
\text { (feet) }\end{array}$ \\
\hline
\end{tabular}

B1-43-6bc. Dug by Kelly Well Co. Inc., 1921

\begin{tabular}{|c|c|c|}
\hline $\begin{array}{l}\text { Soil and sand.......... } \\
\text { Ogallala formation: }\end{array}$ & 12 & 12 \\
\hline 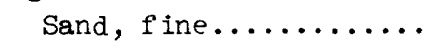 & 40 & 52 \\
\hline Sha le.............. & 1 & 53 \\
\hline $\begin{array}{l}\text { Sand, fine } \ldots \ldots \ldots \ldots \ldots \\
\text { Pierre shale(?): }\end{array}$ & 21 & 74 \\
\hline 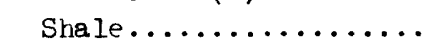 & 2 & 76 \\
\hline
\end{tabular}

\begin{tabular}{|c|c|c|}
\hline \multicolumn{3}{|l|}{$B 1-43-7 a$} \\
\hline $\begin{array}{l}\text { Soil ................. } \\
\text { Ogallala formation: }\end{array}$ & 1 & 1 \\
\hline Rock............. & 9 & 10 \\
\hline Sand............... & 10 & 20 \\
\hline Clay.$\ldots \ldots \ldots \ldots \ldots$ & 11 & 31 \\
\hline Gravel............. & 27 & 58 \\
\hline Clay and gravel....... & 15 & 73 \\
\hline
\end{tabular}


Table 2.--Logs of test holes, seismograph shot holes, and wells--Continued

Yuma County--Continued

\begin{tabular}{|c|c|c|}
\hline & $\begin{array}{c}\text { Thickness } \\
\text { (feet) }\end{array}$ & $\begin{array}{l}\text { Depth } \\
\text { (feet) }\end{array}$ \\
\hline \multicolumn{3}{|c|}{ BI-43-7a--Continued } \\
\hline Ogallala formation--Con. & & \\
\hline Clay...$\ldots \ldots \ldots \ldots$ & 10 & 83 \\
\hline Rock $\ldots \ldots \ldots \ldots \ldots$ & 10 & 93 \\
\hline Clay..$\ldots \ldots \ldots \ldots \ldots$ & 4 & 97 \\
\hline Gravel.............. & 23 & 120 \\
\hline
\end{tabular}

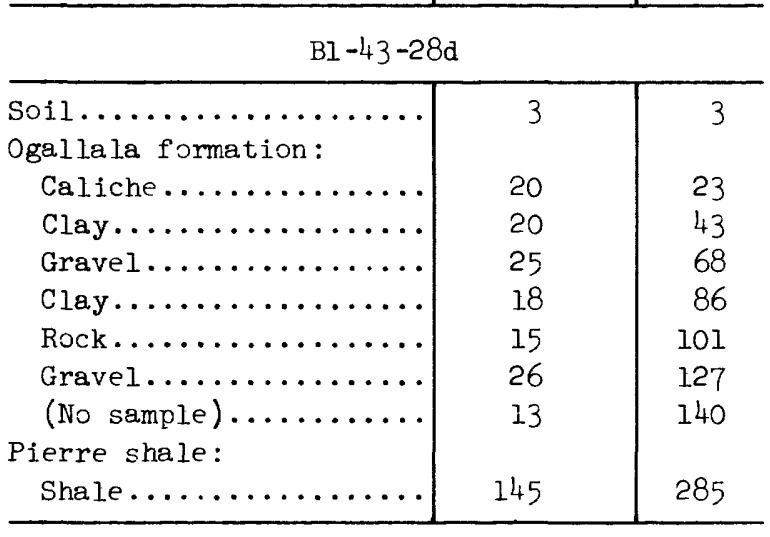

BI $-44-4 a$

\begin{tabular}{|c|c|c|}
\hline 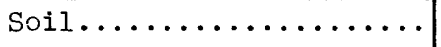 & 2 & 2 \\
\hline Ogallala formation: & & \\
\hline 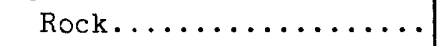 & 2 & 4 \\
\hline 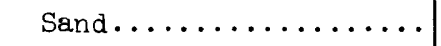 & 17 & 21 \\
\hline Gravel.............. & 14 & 35 \\
\hline 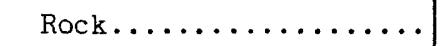 & 5 & 40 \\
\hline Gravel............ & 10 & 50 \\
\hline Rock $\ldots \ldots \ldots \ldots \ldots \ldots$ & 11 & 61 \\
\hline Shale $\ldots \ldots \ldots \ldots \ldots$ & 6 & 67 \\
\hline Gravel............... & 23 & 90 \\
\hline
\end{tabular}

B2 $-42-34 c c$. Drilled by L. L. Canfield, 1949

\begin{tabular}{|c|c|c|}
\hline \multirow{3}{*}{ 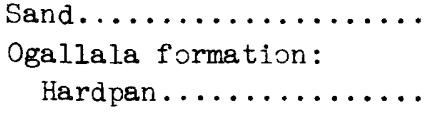 } & 11 & 11 \\
\hline & & \\
\hline & 7 & 18 \\
\hline Clay...$\ldots \ldots \ldots \ldots$ & 16 & 34 \\
\hline Sand, fine...$\ldots \ldots \ldots$ & 7 & 41 \\
\hline Clay...$\ldots \ldots \ldots \ldots$ & 6 & 47 \\
\hline Gravel and sand......... & 9 & 56 \\
\hline Gravel and clay........ & 4 & 60 \\
\hline Gravel, hard, thinly & & \\
\hline bedded..$\ldots \ldots \ldots \ldots$ & 5 & 65 \\
\hline Shale, yellow.......... & 4 & 69 \\
\hline Pierre shale: & & \\
\hline Shale, black......... & 11 & 80 \\
\hline
\end{tabular}

Table 2.--Logs of test holes, seismograph shot holes, and wells--Continued

Yuma County--Continued

\begin{tabular}{|c|c|c|}
\hline & $\begin{array}{c}\text { Thickness } \\
\text { (feet) }\end{array}$ & $\begin{array}{l}\text { Depth } \\
\text { (feet) }\end{array}$ \\
\hline \multicolumn{3}{|c|}{$B 2-44-24 c d$} \\
\hline Soil $\ldots \ldots \ldots \ldots \ldots \ldots$ & 3 & 3 \\
\hline Ogallala formation:- & & \\
\hline 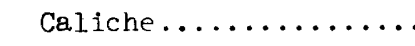 & 8 & 11 \\
\hline Clay.$\ldots \ldots \ldots \ldots \ldots$ & 20 & 31 \\
\hline Gravel............... & 20 & 51 \\
\hline Rock................. & 23 & 74 \\
\hline Gravel............... & 18 & 92 \\
\hline Rock................ & 12 & 104 \\
\hline Gravel............... & 26 & 130 \\
\hline
\end{tabular}

B2-48-15dd. Drilled by William Schocke, 1950

\begin{tabular}{|c|c|c|}
\hline 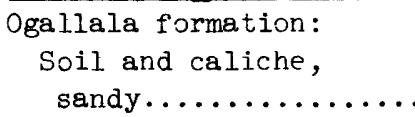 & 60 & 60 \\
\hline 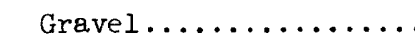 & 10 & 70 \\
\hline Sand $\ldots \ldots \ldots \ldots \ldots \ldots$ & 10 & 80 \\
\hline Silt and gravel, red... & 40 & 120 \\
\hline Sand $\ldots \ldots \ldots \ldots \ldots \ldots$ & 30 & 1.50 \\
\hline Gravel $\ldots \ldots \ldots \ldots \ldots$ & 10 & 160 \\
\hline "Formation," white.... & 20 & 180 \\
\hline $\operatorname{Sand} \ldots \ldots \ldots \ldots \ldots \ldots$ & 10 & 190 \\
\hline Gravel (water)........ & 10 & 200 \\
\hline Silt, red, and gravel. & 30 & 230 \\
\hline Gravel (water)........ & 10 & 240 \\
\hline
\end{tabular}

B2 - $48-22 a c$

\begin{tabular}{|c|c|c|}
\hline 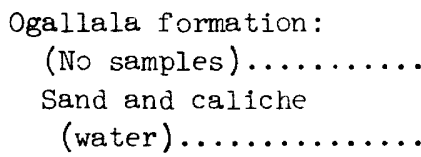 & 200 & 200 \\
\hline Caliche, soft.......... & .5 & $203 \cdot 5$ \\
\hline 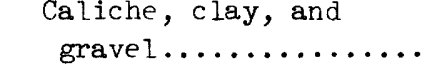 & (?) & (?) \\
\hline $\begin{array}{l}\text { Caliche, soft } \ldots \ldots \ldots \\
\text { Clay and gravel } \ldots \ldots\end{array}$ & $\begin{array}{l}(?) \\
(?)\end{array}$ & $\begin{array}{l}(?) \\
(?)\end{array}$ \\
\hline Caliche, soft......... & (?) & 213 \\
\hline $\begin{array}{l}\text { Clay, very compact...... } \\
\text { Caliche } . . . \ldots \ldots \ldots \ldots\end{array}$ & $\begin{array}{r}9.5 \\
.5\end{array}$ & $\begin{array}{l}222 \cdot 5 \\
223\end{array}$ \\
\hline Clay and sand......... & 4 & 227 \\
\hline 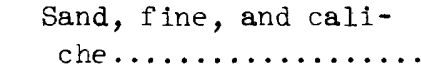 & 13 & 240 \\
\hline Clay and sand...$\ldots \ldots$ & 10 & 250 \\
\hline Caliche and gravel..... & 4 & 254 \\
\hline Caliche and sandstone.. & 1 & 255 \\
\hline Clay, very compact..... & 12 & 261 \\
\hline Clay, heavy, $y \in l l o w . .$. & 10 & 2.7 \\
\hline
\end{tabular}


Table 2.--Logs of test holes, seismograph shot holes, and wells--Continued

Yuma County--Continued

\begin{tabular}{l|c|c}
\hline & $\begin{array}{c}\text { Thickness } \\
\text { (feet) }\end{array}$ & $\begin{array}{c}\text { Depth } \\
\text { (feet) }\end{array}$ \\
\hline
\end{tabular}

B2-48-22ac--Continued

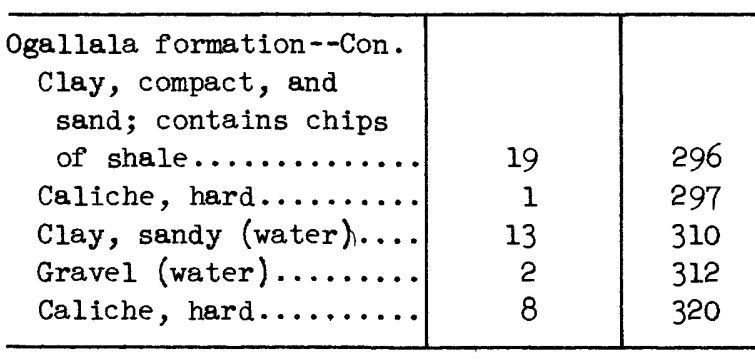

B2-48-22dal. Drilled by Chicago Burlington \& Quincy Railroad Co., 1918

\begin{tabular}{|c|c|c|}
\hline \multicolumn{3}{|l|}{ Ogallala formation: } \\
\hline 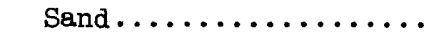 & 45 & 45 \\
\hline Sand, coarse......... & 10 & 55 \\
\hline Clay and sand......... & 20 & 75 \\
\hline 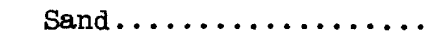 & 4 & 79 \\
\hline 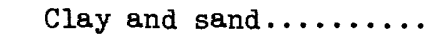 & 30 & 109 \\
\hline Sand and gravel....... & 30 & 139 \\
\hline Sand, fine.......... & 30 & 169 \\
\hline 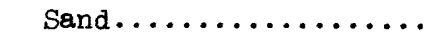 & 6 & 175 \\
\hline Sandstone............ & 3 & 178 \\
\hline 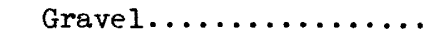 & 30 & 208 \\
\hline Clay and sand......... & 10 & 218 \\
\hline
\end{tabular}

B2-48-22da2. Drilled by Chicago Burlington \& Quincy Railroad Co., 1918

\begin{tabular}{|c|c|c|}
\hline 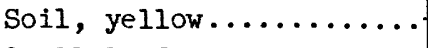 & 15 & 15 \\
\hline Ogallala formation: & & \\
\hline 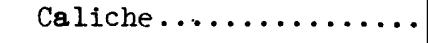 & 25 & 40 \\
\hline Clay and gravel....... & 45 & 85 \\
\hline Quicksand............ & 15 & 100 \\
\hline Clay, yellow.......... & 10 & 110 \\
\hline Clay and boulders..... & 10 & 120 \\
\hline Clay, tough, yellow.... & 10 & 130 \\
\hline Sand, fine........... & 5 & 135 \\
\hline $\begin{array}{l}\text { Sand, coarse and boul- } \\
\text { ders } \ldots \ldots \ldots \ldots \ldots \ldots\end{array}$ & 30 & 165 \\
\hline Quicksand........... & 15 & 180 \\
\hline Sand, coarse (water)... & 10 & 190 \\
\hline 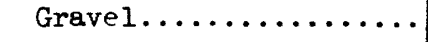 & 12 & 202 \\
\hline
\end{tabular}

\section{B3 $-46-4 b d$}

\begin{tabular}{|c|c|c|}
\hline 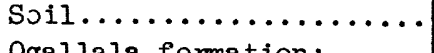 & 2 & 2 \\
\hline Ogallala formation: & & \\
\hline Clay, yellow......... & 18 & 20 \\
\hline Rock, hard.... & 4 & 24 \\
\hline
\end{tabular}

Table 2.--Logs of test holes, seismograph shot holes, and wells--Continued

Yuma County--Continued

\begin{tabular}{|c|c|c|}
\hline & $\begin{array}{c}\text { Thickness } \\
\text { (feet) }\end{array}$ & $\begin{array}{l}\text { Depth } \\
\text { (feet) }\end{array}$ \\
\hline \multicolumn{3}{|c|}{ B3-46-4bd--Continued } \\
\hline Ogallala formation--Con. & & \\
\hline Clay.............. & 20 & 44 \\
\hline Clay and grave $1 . \ldots \ldots$ & 20 & 64 \\
\hline Caliche............. & 28 & 92 \\
\hline Gravel (water)........ & 10 & 102 \\
\hline 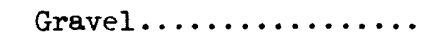 & 39 & 141 \\
\hline
\end{tabular}

B4 $-44-18 \mathrm{db}$

\begin{tabular}{|c|c|c|}
\hline 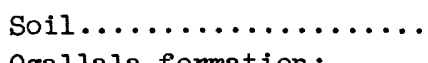 & 2 & 2 \\
\hline 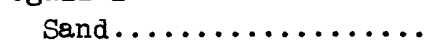 & 18 & 20 \\
\hline Caliche............ & 15 & 35 \\
\hline Grave1............. & 6 & 41 \\
\hline Rock............. & 8 & 49 \\
\hline Sand and gravel....... & 11 & 60 \\
\hline 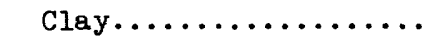 & 12 & 72 \\
\hline 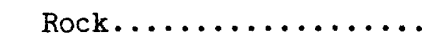 & 5 & 77 \\
\hline Clay.............. & 8 & 85 \\
\hline Grave1............ & 25 & 110 \\
\hline
\end{tabular}

B4-44-36cb. Drilled by L. L. Canfield, 1949

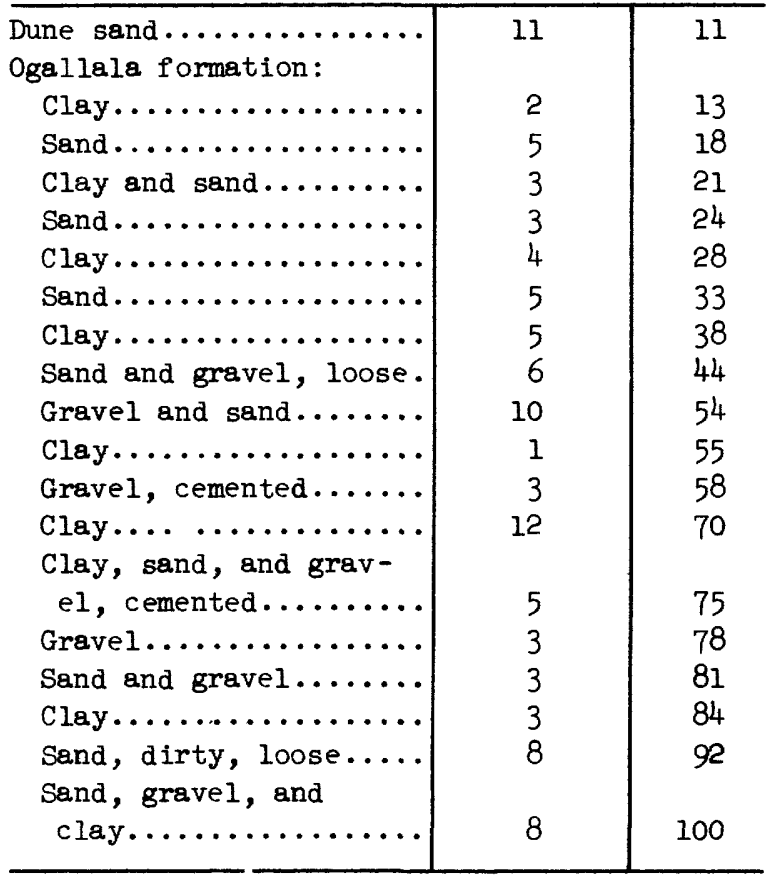


Table 2.--Logs of test holes, seismograph shot holes, and wells--Continued

Yuma County--Continued

\begin{tabular}{l|c|c}
\hline & $\begin{array}{c}\text { Thickness } \\
\text { (feet) }\end{array}$ & $\begin{array}{c}\text { Depth } \\
\text { (feet) }\end{array}$ \\
\hline
\end{tabular}

$\mathrm{B} 4-45-14 \mathrm{cc}$

\begin{tabular}{|c|c|c|}
\hline Soil..................... & 2 & 2 \\
\hline Ogallala formation: & & \\
\hline Sand, white......... & 20 & 22 \\
\hline Gumbo, black.......... & 5 & 27 \\
\hline Sand, white (water).... & 15 & 42 \\
\hline Shale, blue...$\ldots \ldots \ldots$ & 10 & 52 \\
\hline Rock $\ldots \ldots \ldots \ldots \ldots \ldots$ & 20 & 72 \\
\hline Gravel............. & 20 & 92 \\
\hline Gravel (water)......... & 28 & 120 \\
\hline
\end{tabular}

\begin{tabular}{|c|c|c|}
\hline \multicolumn{3}{|c|}{ B4 $-46-5$} \\
\hline Ogallala formation: & & \\
\hline Sand $\ldots \ldots \ldots \ldots \ldots \ldots$ & 20 & 20 \\
\hline Clay, red............. & 20 & 40 \\
\hline Gravel............. & 40 & 80 \\
\hline Clay, red............ & 40 & 120 \\
\hline Gravel........ & 60 & 180 \\
\hline Gravel (water) $\ldots \ldots \ldots$ & 16 & 196 \\
\hline Clay.$\ldots \ldots \ldots \ldots \ldots$ & $\ldots$ & 196 \\
\hline
\end{tabular}

B5-43-24ab. Drilled by L. L. Canfield, 1949

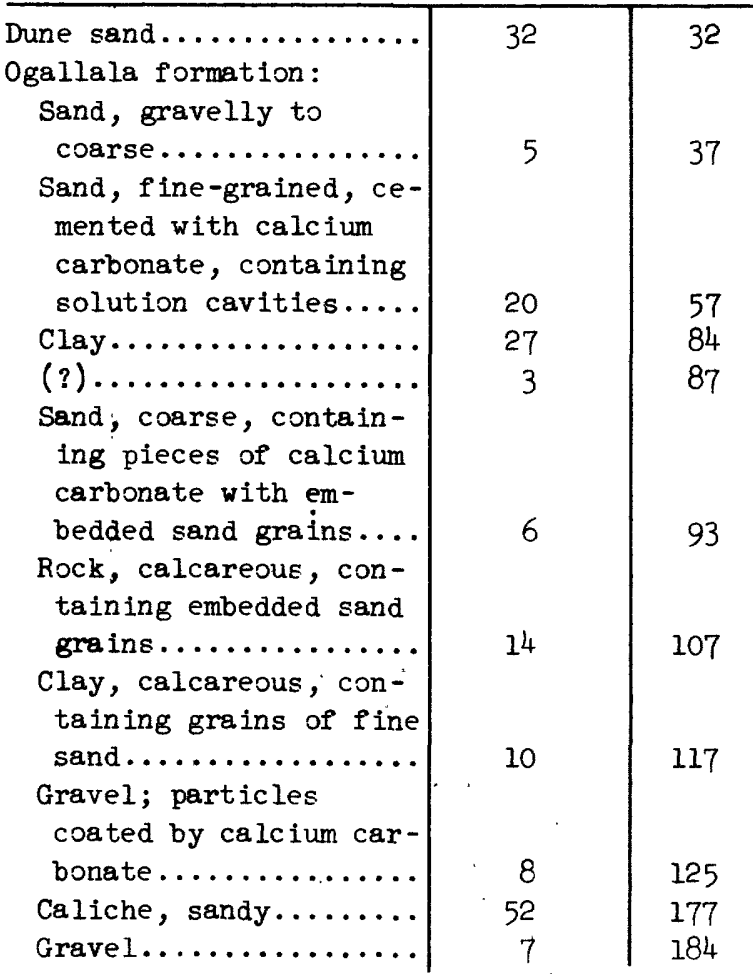

Table 2.--Logs of test holes, seismograph shot holes, and wells--Continued

Yuma County--Continued

\begin{tabular}{l|c|c}
\hline & $\begin{array}{c}\text { Thickness } \\
\text { (feet) }\end{array}$ & $\begin{array}{c}\text { Depth } \\
\text { (feet) }\end{array}$ \\
\hline
\end{tabular}

B5-43-24ab--Continued

\begin{tabular}{|c|c|c|}
\hline $\begin{array}{l}\text { Ogallala formation--Con. } \\
\text { Clay, brown, with. } \\
\text { stringer of caliche.. }\end{array}$ & 13 & 197 \\
\hline Gravel............... & 20 & 217 \\
\hline Caliche, sandy........ & 7 & 224 \\
\hline Gravel................. & 11 & 235 \\
\hline $\begin{array}{l}\text { Clay, tan; contains } \\
\text { fossil seeds........ }\end{array}$ & 5 & 240 \\
\hline $\begin{array}{l}\text { Gravel and sand } \\
\text { coarse } \ldots \ldots \ldots \ldots\end{array}$ & 9 & 249 \\
\hline 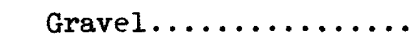 & 11 & 260 \\
\hline
\end{tabular}

\begin{tabular}{|c|c|c|}
\hline \multicolumn{3}{|c|}{ B5-46-1 } \\
\hline Ogallala formation: & & \\
\hline Clay, red, and gravel.. & 150 & 150 \\
\hline Clay, soft, red........ & 50 & 200 \\
\hline Gravel, coarse........ & 10 & 210 \\
\hline Clay $\ldots \ldots \ldots \ldots \ldots \ldots$ & 21 & 231 \\
\hline Gravel, coarse (water). & 18 & 249 \\
\hline
\end{tabular}

\begin{tabular}{|c|c|c|}
\hline \multicolumn{3}{|c|}{ B5 $-48-12$} \\
\hline Loam, black............. & 3 & 3 \\
\hline Ogallala formation: & & \\
\hline Hardpan and gravel.... & 77 & 80 \\
\hline Gravel............. & 80 & 160 \\
\hline Clay and gravel........ & 103 & 263 \\
\hline Gravel, coarse (water). & 3 & 266 \\
\hline
\end{tabular}

\begin{tabular}{|c|c|c|}
\hline \multicolumn{3}{|c|}{$\mathrm{cl}-43-5 \mathrm{~cd}$} \\
\hline Ogallala formation: & & \\
\hline Soil.................. & 160 & 160 \\
\hline Gravel.............. & 52 & 212 \\
\hline Clay and gravel........ & 38 & 250 \\
\hline Pierre shale: & & \\
\hline Shale.......... & 10 & 260 \\
\hline
\end{tabular}

\begin{tabular}{|c|c|c|}
\hline \multicolumn{3}{|c|}{ Cl- $-43-34 \mathrm{bb}$. } \\
\hline 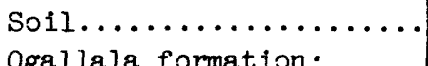 & 3 & 3 \\
\hline 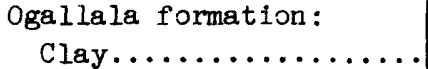 & 15 & 18 \\
\hline 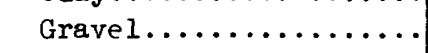 & 3 & 21 \\
\hline Pierre shale: & & \\
\hline Shale $\ldots \ldots \ldots \ldots \ldots \ldots$ & 1 & 22 \\
\hline
\end{tabular}


Table 2.--Logs of test holes, seismograph shot holes, and wells--Continued

Yuma County--Continued

\begin{tabular}{l|l|l}
\hline & $\begin{array}{c}\text { Thickness } \\
\text { (feet) }\end{array}$ & $\begin{array}{c}\text { Depth } \\
\text { (feet) }\end{array}$ \\
\hline
\end{tabular}

C2 $-44-24$ ba

\begin{tabular}{|c|c|c|}
\hline Soil $\ldots \ldots \ldots \ldots \ldots \ldots$ & 3 & 3 \\
\hline Ogallala formation: & & \\
\hline Caliche.............. & 10 & 13 \\
\hline 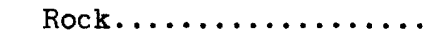 & 15 & 28 \\
\hline Gravel............. & 20 & 48 \\
\hline Clay............... & 20 & 68 \\
\hline Rock............... & 32 & 100 \\
\hline Gravel and sand....... & 30 & 130 \\
\hline Rock.............. & 30 & 160 \\
\hline Pierre shale: & & \\
\hline Shale.............. & 20 & 180 \\
\hline
\end{tabular}

C3-44-28. (South half)

\begin{tabular}{|c|c|c|}
\hline 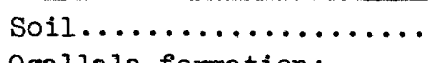 & 2 & 2 \\
\hline $\begin{array}{l}\text { Clay and caliche........ } \\
\text { Gravel (water)......... }\end{array}$ & $\begin{array}{r}240 \\
14\end{array}$ & $\begin{array}{l}242 \\
256\end{array}$ \\
\hline
\end{tabular}

C4-44-5dd. Drilled by Ben Hasz, 1950

\begin{tabular}{|c|c|c|}
\hline $\begin{array}{l}\text { Ogallala formation: } \\
\text { (No samples) .......... }\end{array}$ & 224 & 224 \\
\hline Gravel, good.......... & 23 & 247 \\
\hline 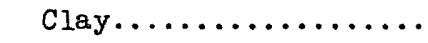 & 1 & 248 \\
\hline $\begin{array}{l}\text { Gravel, good, contain- } \\
\text { ing small strips of }\end{array}$ & & \\
\hline 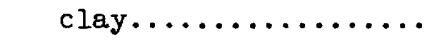 & 7 & 255 \\
\hline $\begin{array}{l}\text { Clay, sandy, with some } \\
\text { sand................ }\end{array}$ & 18 & 273 \\
\hline $\begin{array}{l}\text { Clay, containing strips } \\
\text { of rock.............. }\end{array}$ & 12 & 285 \\
\hline $\begin{array}{l}\text { Sand } . \ldots \ldots \ldots \ldots \ldots \ldots \\
\text { Clay, containing strips }\end{array}$ & 2 & 287 \\
\hline of sand........... & 7 & 294 \\
\hline $\begin{array}{l}\text { Sandstone, interbedded } \\
\text { with clay............ }\end{array}$ & 8 & 302 \\
\hline Sand and gravel, loose. & 13 & 315 \\
\hline $\begin{array}{l}\text { Clay, sandy ............ } \\
\text { Sand, containing small }\end{array}$ & 5 & 320 \\
\hline strips of clay........ & 12 & 332 \\
\hline $\begin{array}{l}\text { Pierre shale }(?): \\
\text { Shale............. }\end{array}$ & 4 & 336 \\
\hline
\end{tabular}

C4-44-10dd. Drilled by K. G. Wilcox, 1950

\begin{tabular}{|c|c|c|}
\hline $\begin{array}{l}\text { Soil.................... } \\
\text { Ogallala formation: }\end{array}$ & 4 & 4 \\
\hline 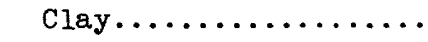 & 52 & 56 \\
\hline
\end{tabular}

Table 2.--Logs of test holes, seismograph shot holes, and wells--Continued

Yuma County--Continued

\begin{tabular}{l|c|c}
\hline & $\begin{array}{c}\text { Thickness } \\
\text { (feet) }\end{array}$ & $\begin{array}{c}\text { Depth } \\
\text { (feet) }\end{array}$ \\
\hline
\end{tabular}

c4-44-10dd--Continued



Ogallala formation-Lon.

Caliche, soft........

Caliche, hard........

Gravel..............

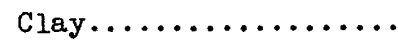

Clay and gravel.......

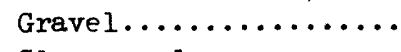

Clay, sandy...........

Gravel..............

Caliche and clay......

Caliche, hard.........

Caliche, hard, and

clay...............

Caliche.............

Clay, sandy...........

Caliche, hard.........

Sandstone and clay.....

Gravel and strips of

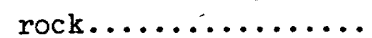

Clay, sandy..........

Gravel..............

Clay and gravel.......

Gravel..............

Caliche.............

Gravel.............

Rock..............

Clay, sandy..........

Rock and hard caliche..

Rock, very hard.......

Clay................

Gravel, coarse, very

loose..............

Sand and clay........

Conglomerate and strips

of clay .............

Conglomerate, hard.....

Clay................

Sandstone...........

Clay, sandy..........

Gravel.............

Clay, sandy..........

Gravel.............

Clay, sandy...........

Gravel, loose, and

strips of clay.......

Clay................

Gravel, medium, loose..

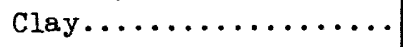

76

86

91

96

108

112

126

137

141

143

154

156

158

159

163

168

170

174

178

184

189

194

196

198

204

213

216

231

236

263

264

265

270

279

282

286

290

292

311

313

326

326 
Table 2.--Logs of test holes, seismograph shot holes, and wells--Continued

Yuma County--Continued

\begin{tabular}{l|l|l}
\hline & $\begin{array}{c}\text { Thickness } \\
\text { (feet) }\end{array}$ & $\begin{array}{c}\text { Depth } \\
\text { (feet) }\end{array}$ \\
\hline
\end{tabular}

C4-47-25dc. Drilled by L. L. Canfield, 1951

\begin{tabular}{|c|c|c|}
\hline Soil $\ldots \ldots \ldots \ldots \ldots \ldots \ldots$ & 5 & 5 \\
\hline Ogallala formation: & & \\
\hline 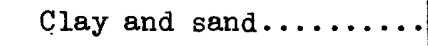 & 4 & 9 \\
\hline Caliche and clay....... & 8 & 17 \\
\hline Gravel and sand........ & 19 & 36 \\
\hline Caliche and gravel.... & 9 & 45 \\
\hline 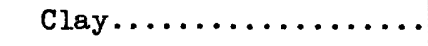 & 10 & 55 \\
\hline Clay and gravel, solid. & 6 & 61 \\
\hline $\begin{array}{l}\text { Clay and sandstone, } \\
\text { thin } . \ldots \ldots \ldots \ldots \ldots \ldots\end{array}$ & 12 & 73 \\
\hline Caliche and sandstone.. & 5 & 78 \\
\hline 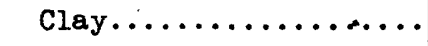 & 4 & 82 \\
\hline Clay and sandstone..... & 14 & 96 \\
\hline $\begin{array}{l}\text { Clay and sandstone; } \\
\text { contains a little }\end{array}$ & & \\
\hline $\begin{array}{l}\text { gravel } \ldots \ldots \ldots \ldots \\
\text { Clay and sandstone..... }\end{array}$ & $\begin{array}{l}4 \\
2\end{array}$ & $\begin{array}{l}100 \\
102\end{array}$ \\
\hline Rock............... & 3 & 105 \\
\hline Clay...$\ldots \ldots \ldots \ldots$ & 1 & 106 \\
\hline 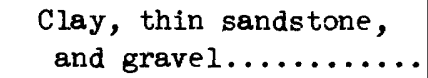 & 6 & 112 \\
\hline $\begin{array}{l}\text { Gravel and sand, solid. } \\
\text { Clay, sandstone and }\end{array}$ & 3 & 115 \\
\hline 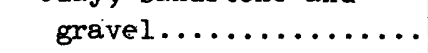 & 3 & 118 \\
\hline Sandstone, solid....... & 2 & 120 \\
\hline Gravel and sand....... & 4 & 124 \\
\hline Sandstone, solid....... & 3 & 127 \\
\hline 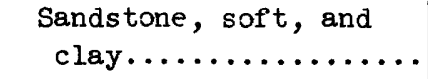 & 6 & 133 \\
\hline $\begin{array}{l}\text { Sandstone, soft and } \\
\text { hard, and clay........ }\end{array}$ & 8 & 141 \\
\hline $\begin{array}{l}\text { Clay, containing thin } \\
\text { strips of soil and } \\
\text { hard sandstone........ }\end{array}$ & 16 & 157 \\
\hline Clay and sand ......... & 1 & 158 \\
\hline $\begin{array}{l}\text { Clay and soft sand- } \\
\text { stone } \ldots \ldots \ldots \ldots \ldots\end{array}$ & 14 & 172 \\
\hline $\begin{array}{l}\text { Clay, sandstone, and } \\
\text { sand, interbedded..... }\end{array}$ & 10 & 182 \\
\hline $\begin{array}{l}\text { Gravel and sand, loose } \\
\text { and tight............ }\end{array}$ & 15 & 197 \\
\hline 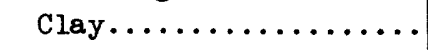 & 4 & 201 \\
\hline 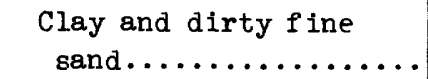 & 3 & 204 \\
\hline 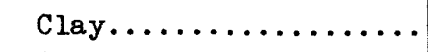 & 5 & 209 \\
\hline $\begin{array}{l}\text { Clay, containing thin } \\
\text { strips of sand......... }\end{array}$ & 7 & 216 \\
\hline
\end{tabular}

Table 2.--Logs of test holes, seismograph shot holes, and wells--Continued

Yuma County--Continued

\begin{tabular}{|c|c|c|}
\hline . & $\begin{array}{c}\text { Thickness } \\
\text { (feet) }\end{array}$ & $\begin{array}{l}\text { Depth } \\
\text { (feet) }\end{array}$ \\
\hline \multicolumn{3}{|c|}{ C4-47-25dc--Continued } \\
\hline $\begin{array}{l}\text { Ogallala formation--Con. } \\
\text { Clay, sand, and gravel } \\
\text { Clay................ } \\
\text { Gravel, coarse, and } \\
\text { fairly loose sand.... } \\
\text { Clay, containing thin } \\
\text { strips of fine sand.. } \\
\text { Gravel, coarse, tight, } \\
\text { and sand; contains a } \\
\text { little clay........ } \\
\text { Shale, yellow....... } \\
\text { Shale; yellow to blue. } \\
\text { Pierre shale: } \\
\text { Shale, blue.......... }\end{array}$ & $\begin{array}{r}4 \\
5 \\
16\end{array}$ & $\begin{array}{l}265 \\
274 \\
287 \\
\\
288\end{array}$ \\
\hline
\end{tabular}

C5 $-42-5$. (East half)

\begin{tabular}{|c|c|c|}
\hline \multirow{2}{*}{$\begin{array}{l}\text { Soil.................. } \\
\text { Ogallala formation: }\end{array}$} & \multirow[t]{2}{*}{20} & \multirow[t]{2}{*}{20} \\
\hline & & \\
\hline Rock.$\ldots \ldots \ldots \ldots$ & 4 & 24 \\
\hline Clay, sandy........... & 4 & 28 \\
\hline Sand (water) ..... & 4 & 32 \\
\hline Gravel (water)..... & 6 & 38 \\
\hline Clay balls and gravel. & 9 & 47 \\
\hline Gravel (water)........... & 4 & 51 \\
\hline Pierre shale(?): & & \\
\hline 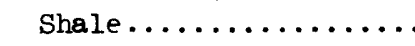 & $\ldots$ & 51 \\
\hline
\end{tabular}

C5-44-23dd. Drilled by K. G. Wilcox, 1950

\begin{tabular}{|c|c|c|}
\hline Soil $\ldots \ldots \ldots \ldots \ldots \ldots$ & 5 & 5 \\
\hline Ogallala formation: & & \\
\hline $\begin{array}{l}\text { Clay and gravel; con- } \\
\text { tains soft rock........ }\end{array}$ & 2 & 7 \\
\hline $\begin{array}{l}\text { Clay, containing strips } \\
\text { of gravel and rock.... }\end{array}$ & 14 & 21 \\
\hline $\begin{array}{l}\text { Clay and gravel........ } \\
\text { Gravel, coarse, very }\end{array}$ & 9 & 30 \\
\hline loose $\ldots \ldots \ldots \ldots \ldots$ & 11 & 41 \\
\hline Clay...$\ldots \ldots \ldots \ldots$ & 1 & 42 \\
\hline 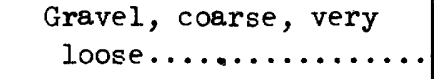 & 5 & 47 \\
\hline $\begin{array}{l}\text { Sand and gravel, loose; } \\
\text { contains strips of }\end{array}$ & & \\
\hline $\begin{array}{l}\text { clay .................. } \\
\text { contains strips of }\end{array}$ & 5 & 52 \\
\hline clay........... & 23 & 75 \\
\hline
\end{tabular}


Table 2.--Logs of test holes, seismograph shot holes, and wells--Continued

Yuma County--Continued

\begin{tabular}{|c|c|c|}
\hline . & $\begin{array}{c}\text { Thickness } \\
\text { (feet) }\end{array}$ & $\begin{array}{l}\text { Depth } \\
\text { (feet) }\end{array}$ \\
\hline \multicolumn{3}{|c|}{ C5-44-23dd--Continued } \\
\hline $\begin{array}{l}\text { Pierre shale: } \\
\text { Shale, yellow......... } \\
\text { Shale, blue......... }\end{array}$ & $\begin{array}{l}5 \\
5\end{array}$ & $\begin{array}{l}80 \\
85\end{array}$ \\
\hline
\end{tabular}

C5-44-30bb. Drilled by K. G. Wilcox, 1948

\begin{tabular}{|c|c|c|}
\hline 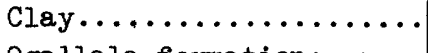 & 21 & 21 \\
\hline Ogallala formation: & & \\
\hline Gravel............. & 10 & 31 \\
\hline 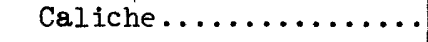 & 6 & 37 \\
\hline 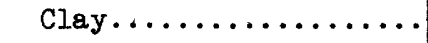 & 6 & 43 \\
\hline Gravel, coarse........ & 10.5 & $53 \cdot 5$ \\
\hline Caliche, soft......... & 4.5 & 58 \\
\hline 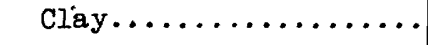 & 2 & 60 \\
\hline 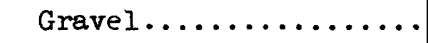 & 3 & 63 \\
\hline Sands tone............ & 1 & 64 \\
\hline Gravel.............. & 21 & 85 \\
\hline Pierre shale(?): & & \\
\hline 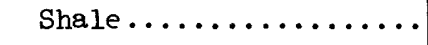 & $\cdots$ & 85 \\
\hline
\end{tabular}

C5-47-4ba. Drilled by Mr.. Skidmore, 1949

\begin{tabular}{|c|c|c|}
\hline 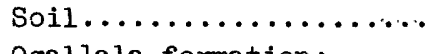 & 10 & 10 \\
\hline Ogallala formation: & & \\
\hline Caliche............. & 4 & 14 \\
\hline $\begin{array}{l}\text { Gravel, clay, and } \\
\text { strips of soft sand- } \\
\text { stone } \ldots \ldots \ldots \ldots \ldots \ldots \ldots\end{array}$ & 92 & 106 \\
\hline Gravel............ & 12 & 118 \\
\hline Gravel and sandstone... & 43 & 161 \\
\hline 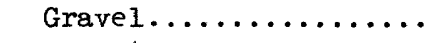 & 12 & 173 \\
\hline Pierre shale: & & \\
\hline Shale, clayey, blue... & 3 & 176 \\
\hline
\end{tabular}

Table 2.--Logs of test holes, seismograph shot holes, and wells--Continued

Yuma County--Continued

\begin{tabular}{l|c|c}
\hline & $\begin{array}{c}\text { Thickness } \\
\text { (feet) }\end{array}$ & $\begin{array}{c}\text { Depth } \\
\text { (feet) }\end{array}$ \\
\hline
\end{tabular}

C5-47-16ad. Drilled by K. G. Wilcox, 1949

\begin{tabular}{|c|c|c|}
\hline \multirow{3}{*}{$\begin{array}{l}\text { Soil.................. } \\
\text { Ogallala formation: } \\
\text { Clay, yellow.......... }\end{array}$} & 3 & 3 \\
\hline & & \\
\hline & 15 & 18 \\
\hline 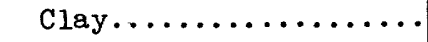 & 5 & 23 \\
\hline 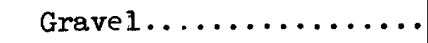 & 13 & 36 \\
\hline 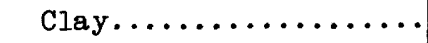 & 7 & 43 \\
\hline 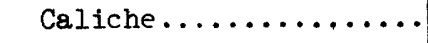 & 14 & 57 \\
\hline Sandstone, soft........ & 5 & 62 \\
\hline Conglomerate......... & 2 & 64 \\
\hline Sand and gravel....... & 13 & 77 \\
\hline Sandstone............ & 6 & 83 \\
\hline Sand, containing strips & & \\
\hline 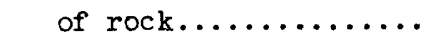 & 4 & 87 \\
\hline Sands tone............ & 7 & 94 \\
\hline Sand and grave1....... & 13 & 107 \\
\hline Gravel.............. & 7 & 114 \\
\hline Clay, sandy .......... & 3 & 117 \\
\hline 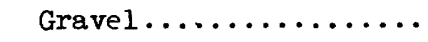 & 12 & 129 \\
\hline Joint clay........... & 28 & 157 \\
\hline 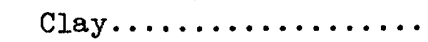 & 3 & 160 \\
\hline $\begin{array}{l}\text { Sand, fine, containing } \\
\text { strips of clay and }\end{array}$ & & \\
\hline 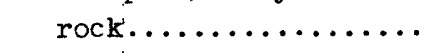 & 8 & 168 \\
\hline Clay, sandy........... & 1 & 169 \\
\hline Sandstone ........... & 1 & 170 \\
\hline $\begin{array}{l}\text { Sand, fine, containing } \\
\text { strips of sandy clay.. }\end{array}$ & 7 & 177 \\
\hline Clay, sandy.......... & 7 & 184 \\
\hline $\begin{array}{l}\text { Sand and gravel, loose. } \\
\text { Clay, sandy, and grav- }\end{array}$ & 14 & 198 \\
\hline 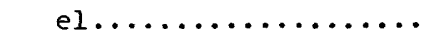 & 1 & 199 \\
\hline Sand and gravel, loose. & 15 & 214 \\
\hline 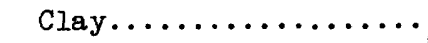 & 1 & 215 \\
\hline
\end{tabular}

